\title{
A CROSSCUTTING THREE-MODES-OF-OPERATION UNIQUE LiDAR-BASED 3D MAPPING SYSTEM \\ GENERIC FRAMEWORK ARCHITECTURE, UNCERTAINTY PREDICTIVE MODEL AND SFM AUGMENTATION
}

\author{
by \\ Ashraf Mohamed Abdelaziz Elshorbagy \\ B.Sc., Cairo University, Egypt, 2000 \\ M.Sc. (Hons), The American University in Cairo, Egypt, 2013 \\ A dissertation \\ presented to Ryerson University \\ in partial fulfillment of the \\ requirements for the degree of \\ Doctor of Philosophy \\ in the program of \\ Civil Engineering
}

Toronto, Ontario, Canada, 2020

(C) Ashraf Elshorbagy, 2020 
I hereby declare that I am the sole author of this dissertation. This is a true copy of the dissertation, including any required final revisions, as accepted by my examiners.

I authorize Ryerson University to lend this dissertation to other institutions or individuals for the purpose of scholarly research.

I further authorize Ryerson University to reproduce this dissertation by photocopying or by other means, in total or in part, at the request of other institutions or individuals for the purpose of scholarly research.

I understand that my dissertation may be made electronically available to the public. 


\title{
A CROSSCUTTING THREE-MODES-OF-OPERATION UNIQUE LiDAR-BASED 3D MAPPING SYSTEM \\ GENERIC FRAMEWORK ARCHITECTURE, UNCERTAINTY PREDICTIVE MODEL AND SfM AUGMENTATION
}

\author{
Doctor of philosophy 2020 \\ Ashraf Elshorbagy \\ Department of Civil Engineering \\ Ryerson University
}

\begin{abstract}
The need for 3D mapping is on the rise to meet the requirements of a growing and diverse group of end-users. Existing 3D mapping systems, which have been classified according to the mode of operation as stationary, mobile and aerial, tend to serve one mode of operation only and are considered cost-prohibitive for many end-users. Unmanned aerial vehicles (UAVs) have experienced rapid growth since their introduction and their usage in 3D mapping is likewise accelerating at a rapid pace. This dissertation presents the design, development and implementation of a LiDAR-based generic 3D mapping system that can be used in the three mapping modes (stationary, mobile and UAV-based). The system provides direct georeferencing capabilities through optimized selected multimodal sensors. A fundamental part of this dissertation is the smart integration of the 3D mapping system components both on the hardware and software levels, along with a new mapping scheme that enables platform-independent deployment ability. This research project also presents a rigorous non-linear uncertainty predictive model for the generic developed system and introduces a very low-cost variant of the system to be used in stationary and handheld mode. The developed multipurpose mapping system is tested in different environments for the three modes of operation, demonstrating its practicality, versatility and ease
\end{abstract}


of deployment. To maximize the ease of deployment for diverse end-users, careful consideration is given to the mapping system components so that the developed system is ultra-lightweight, compact, and multipurpose. Additionally, this dissertation proposes a colorization workflow to make use of available optical imagery in the colorization process of the LiDAR point cloud. Lastly, the study compares two different 3D mapping approaches: 3D LiDAR-based mapping and a lowcost optical-based 3D structure from motion (SfM) workflow. The comparison is achieved by performing a real-world case study of digital surface model (DSM) generation by the two aforementioned approaches. Real-world testing that includes qualitative and quantitative validation against accurate state-of-the-art high-end LiDAR equipment proves the successful design, development and deployment of the developed crosscutting LiDAR-based 3D mapping system. 


\section{ACKNOWLEDGMENTS}

First and foremost, to my advisor, Dr. Ahmed Shaker, I wish to state unequivocally that this research would not have been possible without your continuous support, encouragement and guidance. I really appreciate what you have done for me. Your constant support is very much valued and recognized. I also extend special thanks and acknowledgments to my colleague Hamdy Elsayed. Thanks also go to my colleagues Ebraheem Alhomodi, Yasmine Megahed and Abdelsatar Elmezayen. I would like to extend my thanks to Gemy in the Ryerson collaboratory. My gratitude and thanks also goes to Ahmed Elshorbagy for our insightful discussions. My sincere gratitude goes to my professors Prof. Ahmed El-Rabbany, Prof. Michael Chapman, and Prof. Songnian Li. My thanks also goes to Rachel Peluso and Domenic Valle. 


\section{DEDICATION}

Praise be to God for giving me strength to finish this research. To my late father, I still miss you, DAD, and wish you were here. I learned a lot from you. You are my role model. May God bless your soul. To my wonderful mother, I wish you all the best in your life. To my beloved wife, Eman

Saad, you are my sunshine. To my lovely daughters, Lana and Lilly, you are my princesses; your smile heal whatever weariness I feel. To my great brothers, Mostafa, Ahmed and Mahmoud, I love you all. To Aunt Sal, Magda, Uncle Nagi, and all my family - thank you! 


\section{TABLE OF CONTENTS}

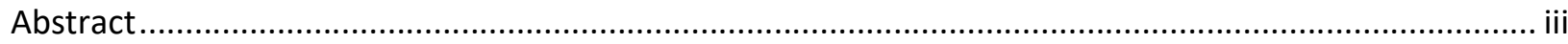

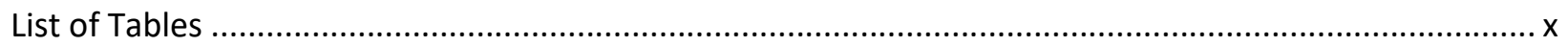

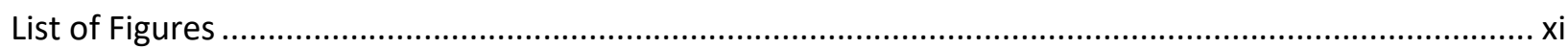

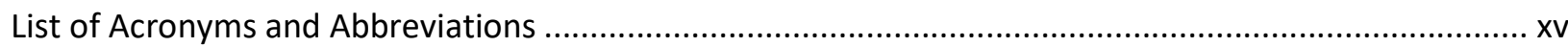

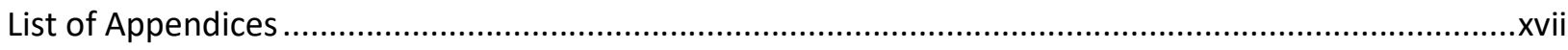

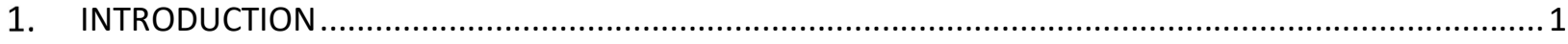

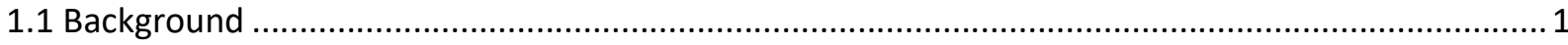

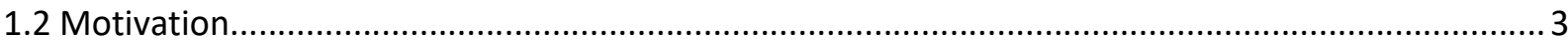

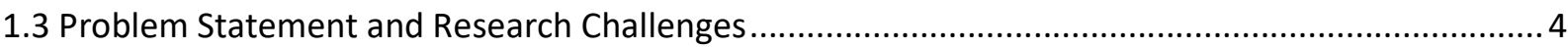

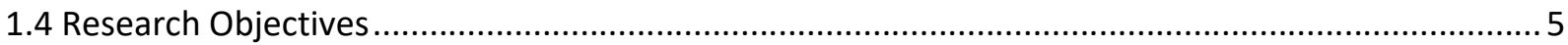

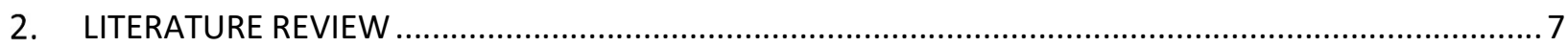

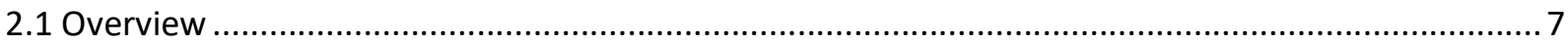

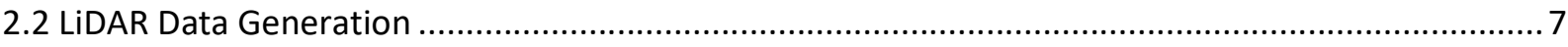

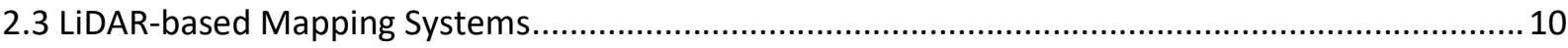

2.4 System orientation impact on the generated point cloud............................................................. 18

2.5 Structure from Motion SfM Photogrammetry....................................................................... 19

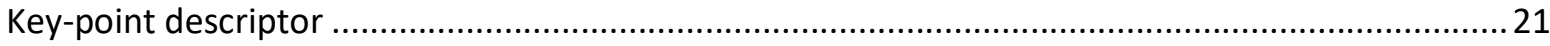

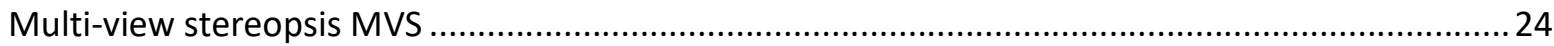

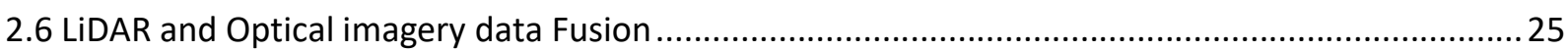

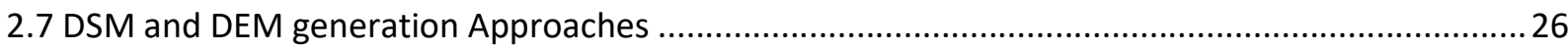

3. LIDAR-BASED PLATFORM-INDEPENDENT MAPPING SYSTEM ARCHITECTURE DEVELOPMENT .........29

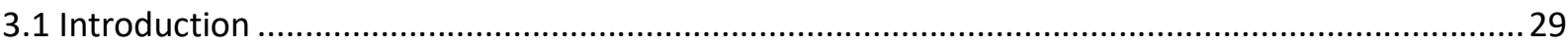

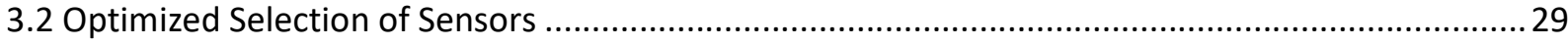

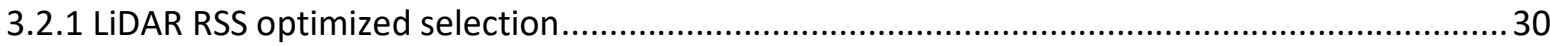

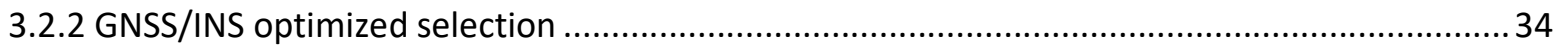

3.2.3 System Management Unit SMU optimized selection .......................................................... 37

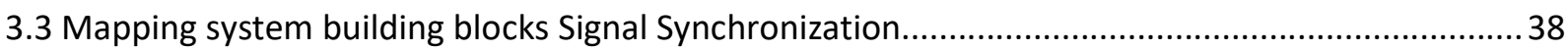

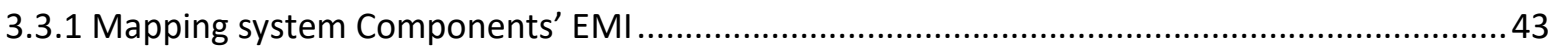

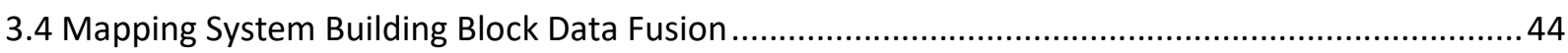

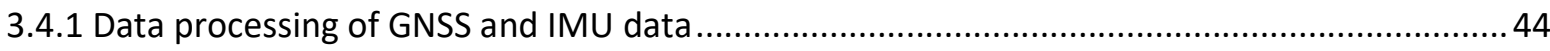

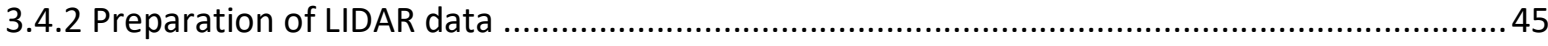

3.4.3 Interpolation and joining with synchronized time-stamps................................................. 45 
4. THE MAPPING SYSTEM'S DIFFERENT MODES OF OPERATION AND THE UNCERTAINTY PREDICTIVE MODEL

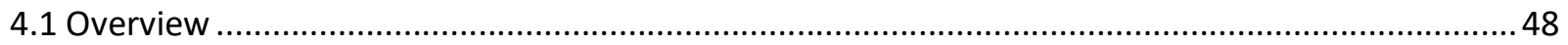

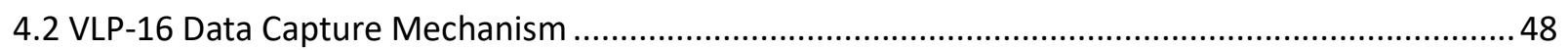

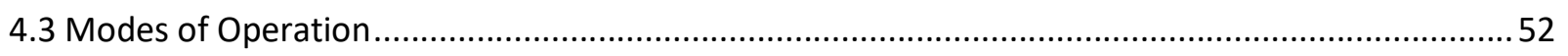

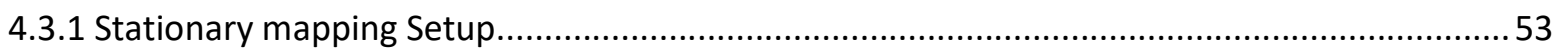

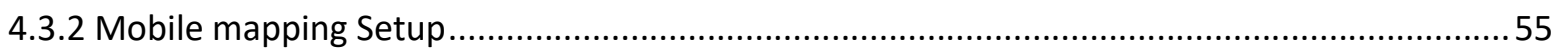

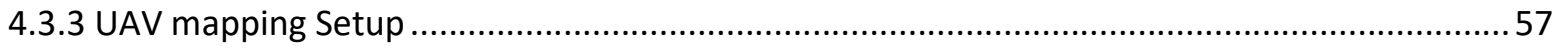

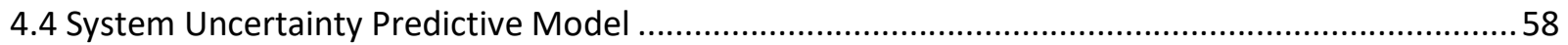

5. MAPPING SYSTEM REALIZATION: STATIONARY, MOBILE MODES OF OPERATION, AND POINT CLOUD

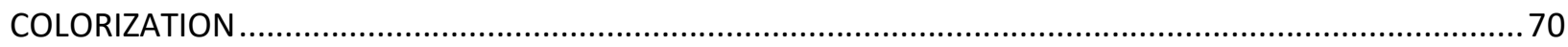

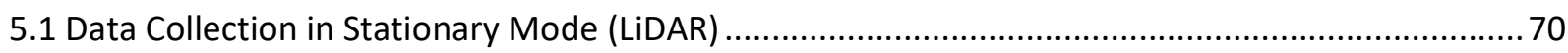

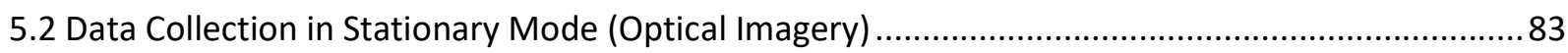

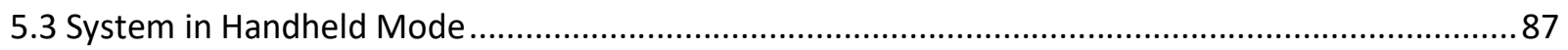

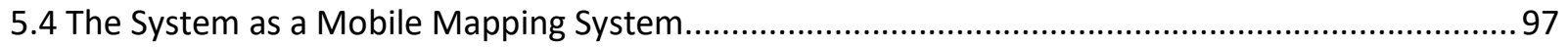

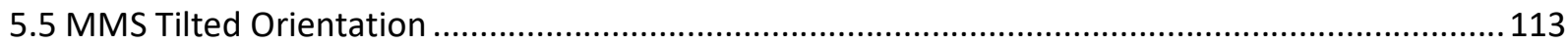

6. THE MAPPING SYSTEM REALIZATION: UAV MODE OF OPERATION, SFM AND DSM GENERATION.. 119

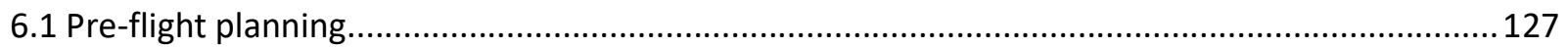

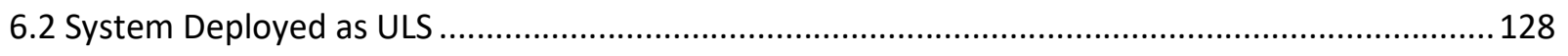

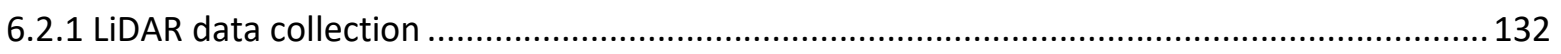

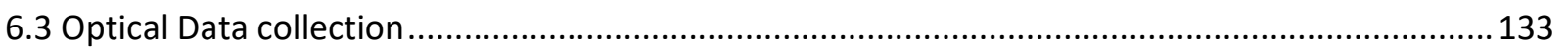

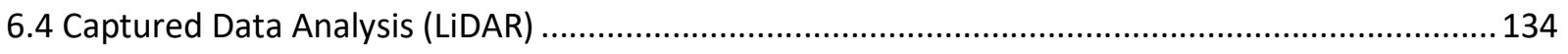

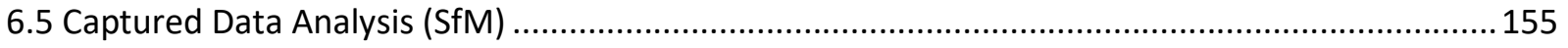

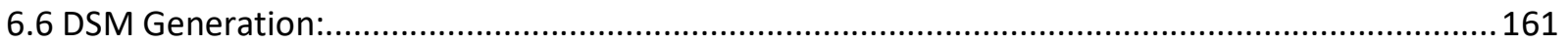

7. SUMMARY, CONCOLUSIONS, KEY CONTRIBUTIONS, AND RECOMMENDATIONS FOR FUTURE

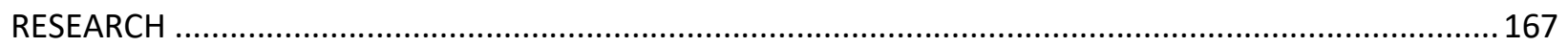

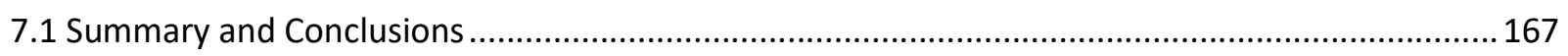

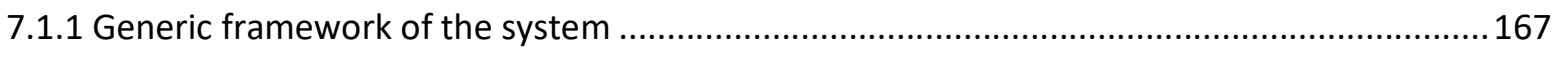

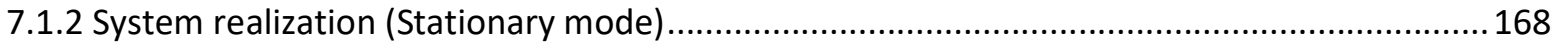

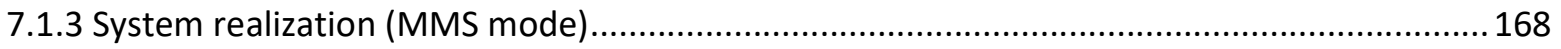

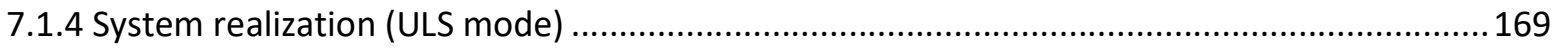

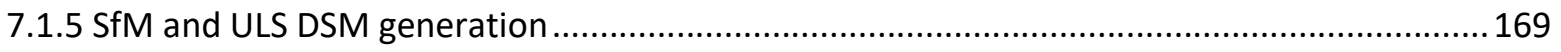

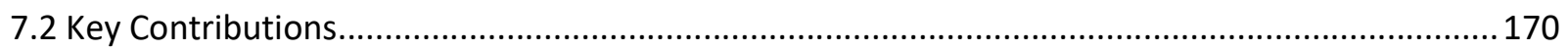

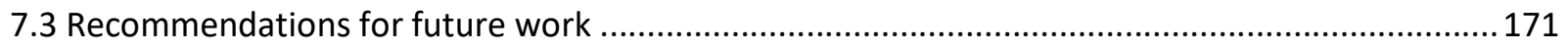




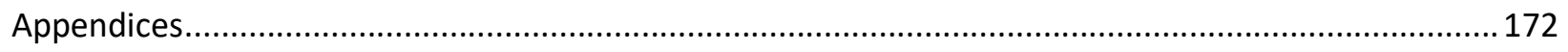

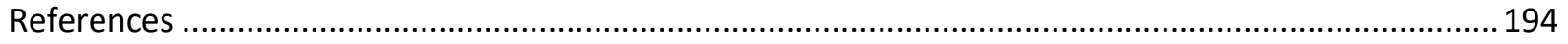




\section{List of Tables}

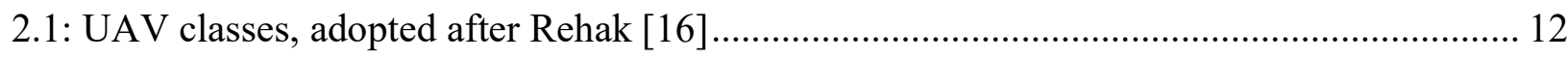

2.2: Comparison of different UAV types, adopted after Chapman [17]...................................... 13

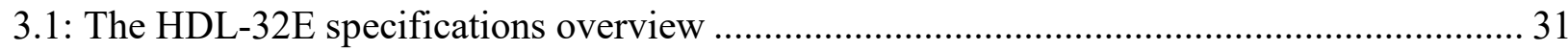

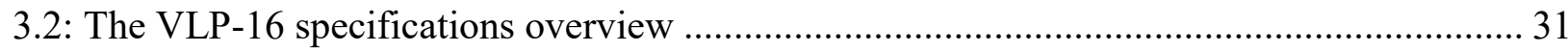

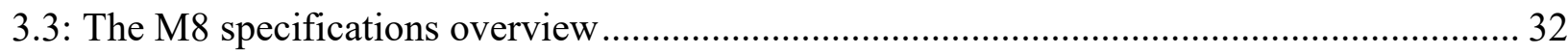

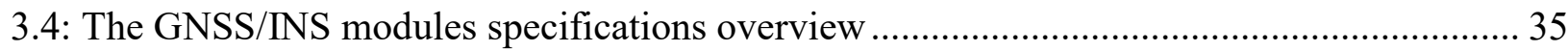

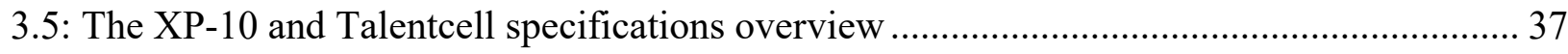

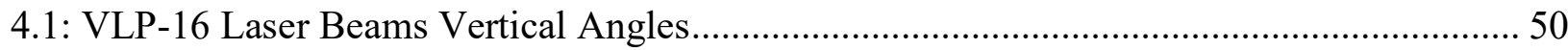

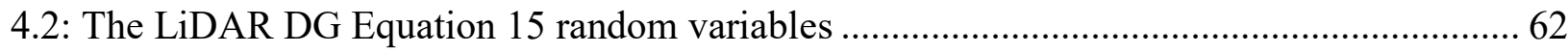

4.3: The 15 random variables used in the error propagation and their initial values......................6 63

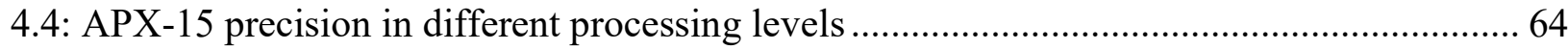

4.5: The System favorable precision (post-processed) \& least favorable SPS ................................. 65

4.6: The system performance metrics (m), @ ranges $25 \mathrm{~m}, 50 \mathrm{~m}, 100 \mathrm{~m}$ (favorable / least favorable scenario; diagonal elements uncertainty in the $X p Y p Z p$ georeferenced coordinates respectively)

4.7: The system performance metrics (m), @ ranges 25m, 50m, 100m (favorable/ least favorable scenario; uncertainty in the $X p Y p Z p$ georeferenced coordinates including beam divergence

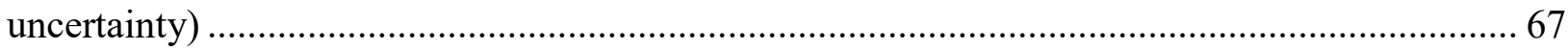

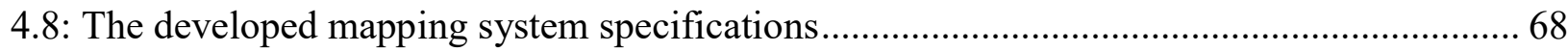

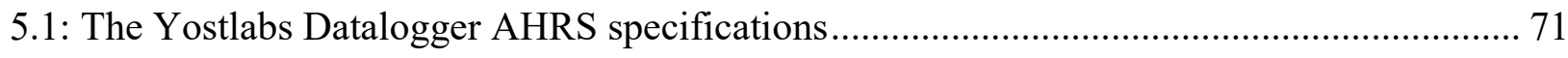

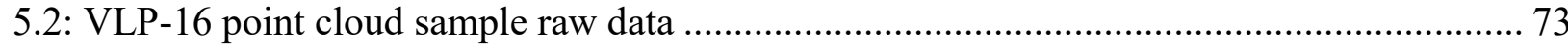

5.3: Sample Measurements (point cloud-ground truth) ………………….................................. 78

5.4: Cellphone camera specs used to capture EPH façade imagery ............................................. 83

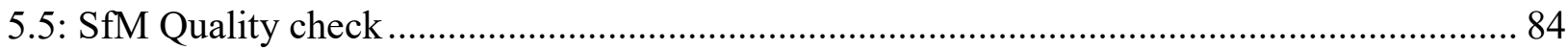

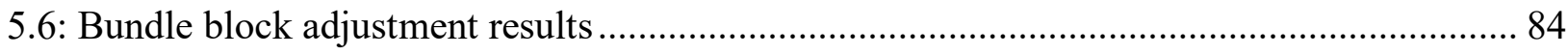

5.7: 3D points calculated from 2D key points matches .......................................................... 84

5.8: The summary of the Aerial LiDAR mission used for the quantitative validation.................. 94

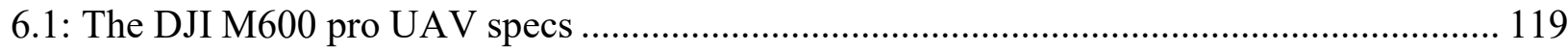

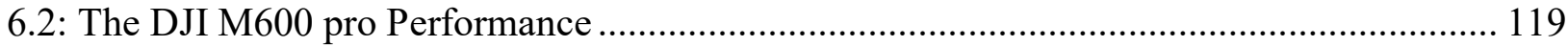

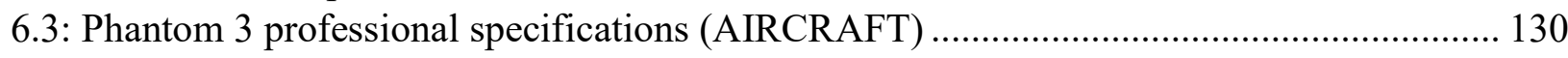

6.4: Phantom 3 professional specifications (Camera) …………………………………....... 130

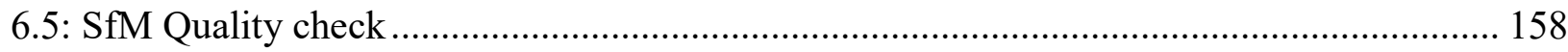

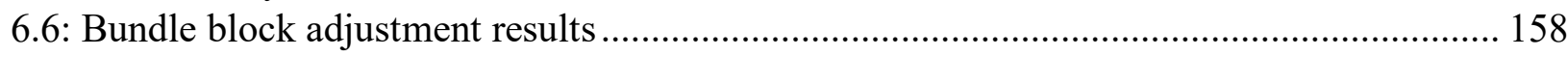

6.7: 3D points calculated from 2D key points matches ..................................................... 158 


\section{List of Figures}

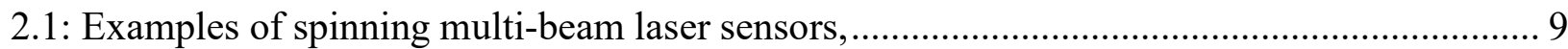

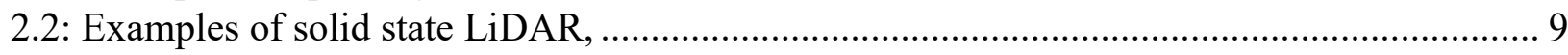

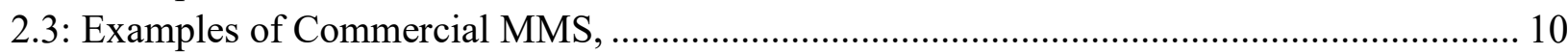

2.4: Examples of recently developed commercial MMS, ....................................................... 11

2.5: Examples of different UAVs types, Multirotor a) DJI M600, b) Topcon Falcon ................. 14

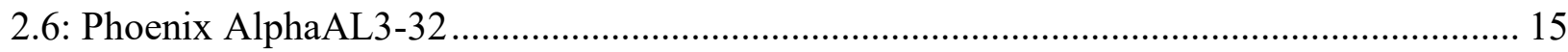

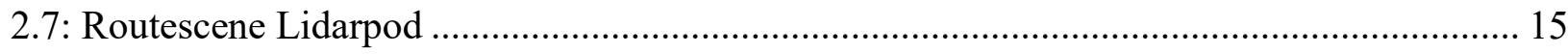

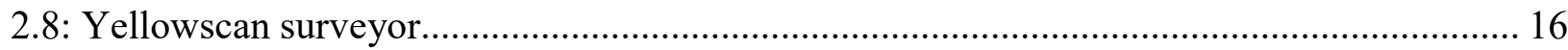

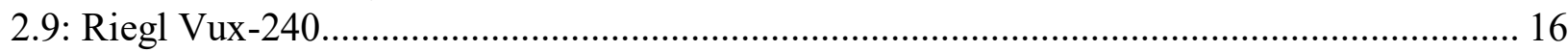

2.10: Examples of different STLS, a) Trimble SX10, b) Faro Focus-350.................................... 17

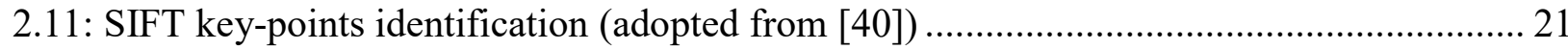

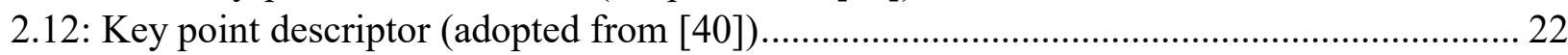

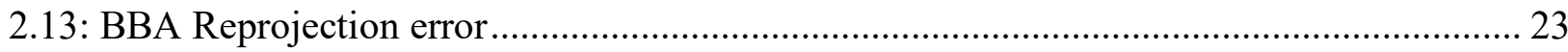

3.1: Examples of SMbL scanners, a) Velodyne HDL-32E, b) Quanergy M8 …………….......... 33

3.2: Examples of GNSS/INS Modules, a) Ellipse2-D, b) PwrPak7D-E1, c) Quanta UAV ......... 36

3.3: Signal Synchronization between the VLP-16 and APX-15 …………................................ 38

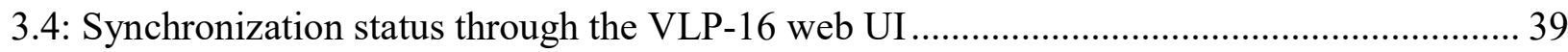

3.5: Synchronization status through Wireshark and position packet............................................. 40

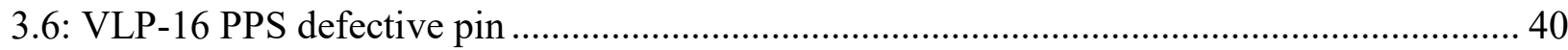

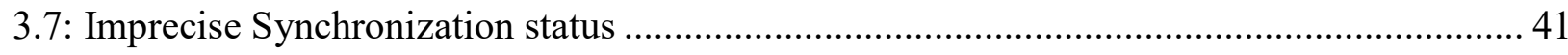

3.8: Mapping system building blocks Integration Scheme ………………………….............. 42

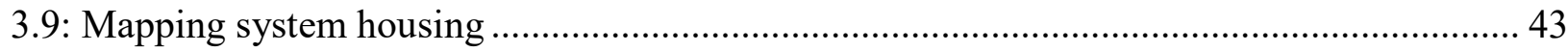

3.10: Workflow for the sensor data fusion........................................................................... 47

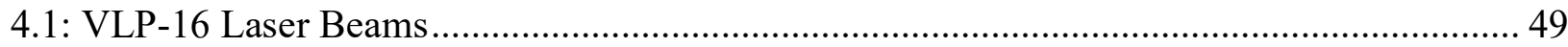

4.2: VLP-16 Axes; Horizontal and Vertical angles .................................................................. 51

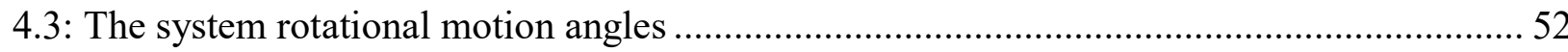

4.4: System Operation Method - Stationary Setup ................................................................. 54

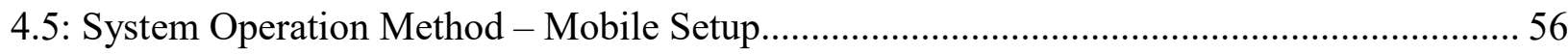

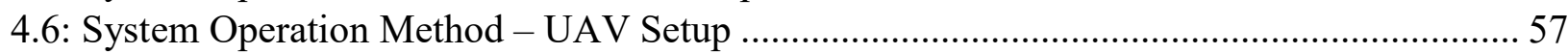

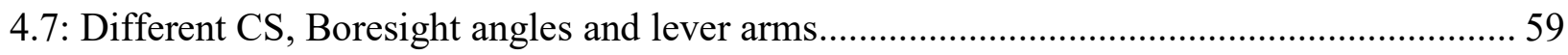

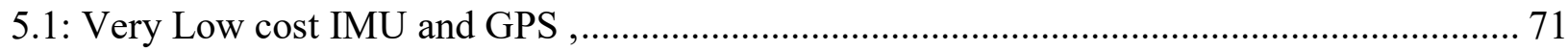

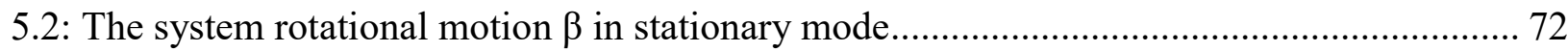

5.3: Original VLP-16 scan with vertical coarse resolution and limited FOV ............................... 74

5.4: EPH building processed point cloud and the corresponding optical imagery ........................ 75

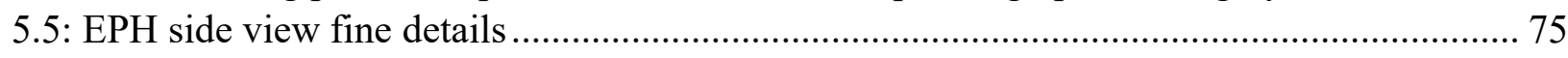

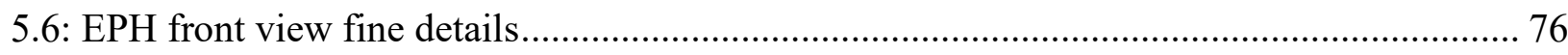

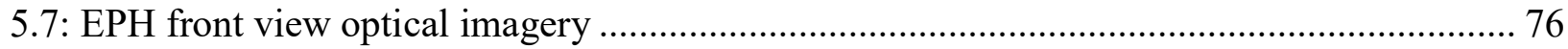

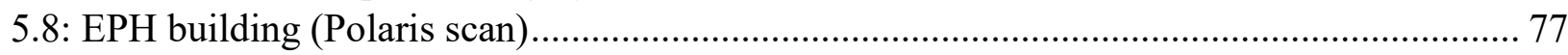

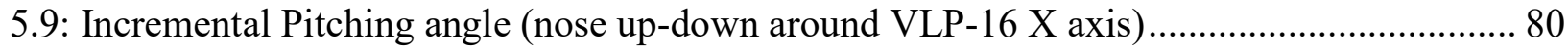

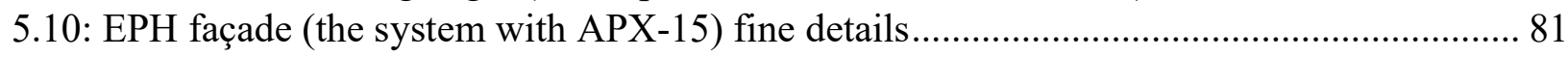

5.11: EPH façade SfM point cloud (images positions are the green circles)................................ 83 
5.12: Average automatic tie points ATP (darker color represents less points found) ............... 85

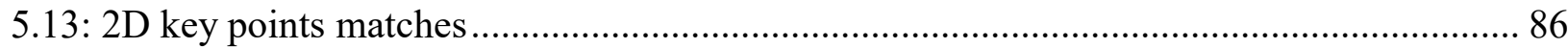

5.14: A satellite image; Gould St and Church St intersection ............................................ 88

5.15: A street-view image; Gould St and Church St intersection ........................................... 88

5.16: Gould St mapped with yostlabs AHRS and Garmin GPS 18x LVC (All frames)............. 89

5.17: Gould St mapped with yostlabs AHRS and Garmin GPS 18x LVC (One frame)............. 89

5.18: Gould St mapped with yostlabs AHRS and Garmin GPS 18x LVC Frames; \#50(blue)

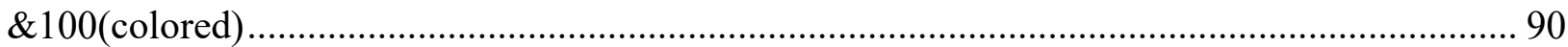

5.19: Gould St mapped with yostlabs AHRS and Garmin GPS 18x LVC (Every other 50th

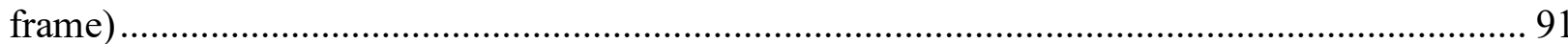

5.20: Gould St mapped with yostlabs AHRS and Garmin GPS 18x LVC (Every other 100th

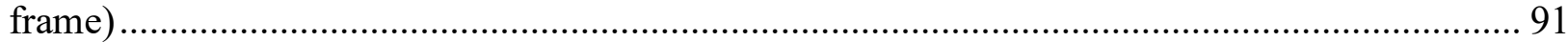

5.21: Gould St mapped with yostlabs AHRS and Garmin GPS 18x LVC .............................. 94

5.22: Deviation of the GPS readings to the accurate LIDAR ground truth .............................. 96

5.23: Timeline for the hardware integration a) Initial testing, b) Prototype version 1 ............... 98

5.24: System Car mounting a) initial b) practical and efficient ............................................. 99

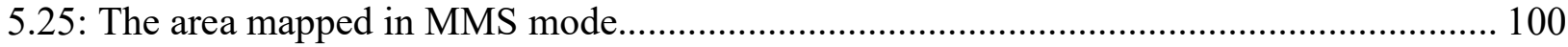

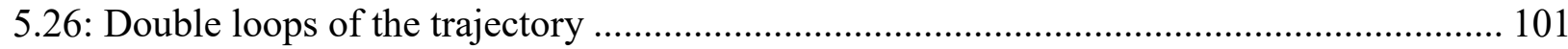

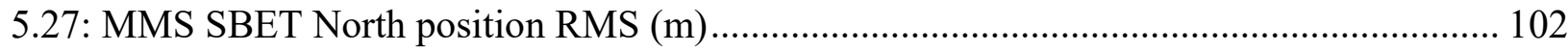

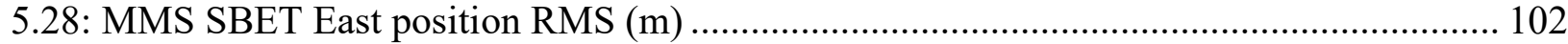

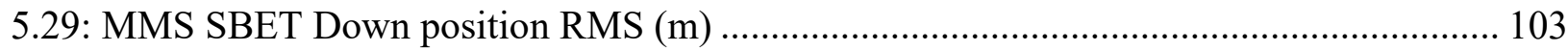

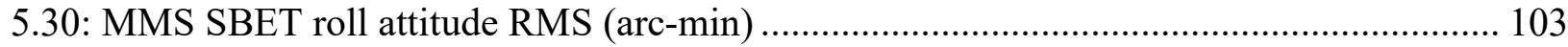

5.31: MMS SBET pitch attitude RMS (arc-min) ............................................................ 104

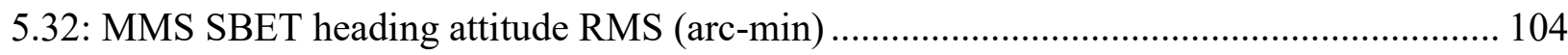

5.33: Aerial LiDAR data coverage (color coded by height) .................................................. 106

5.34: Aerial LiDAR data coverage (Missing areas); ........................................................... 106

5.35: Data mapped by the developed system \& Aerial LiDAR data coverage (color coded by

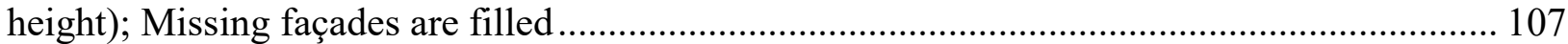

5.36: Aerial LiDAR data coverage 2 (color coded by height); Missing façades are white ........ 107

5.37: Aerial LiDAR data coverage 2 (color coded by height); Missing façades are filled......... 108

5.38: Aerial LiDAR data and the orthophoto deviations (same building side mapped by the

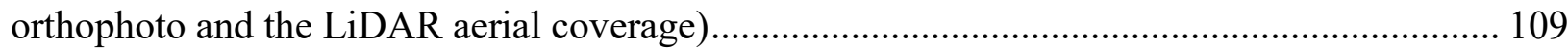

5.39: MMS data no deviation with Aerial LiDAR data; the orthophoto as background ............ 109

5.40: Area coverage (Aerial LiDAR only) ............................................................................ 110

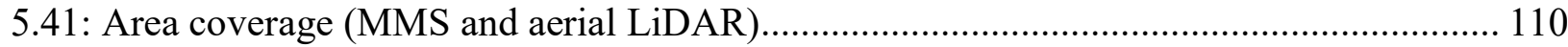

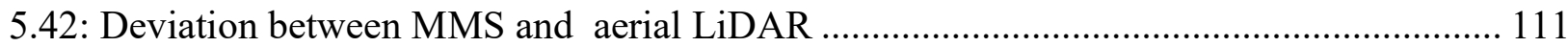

5.43: MMS \& aerial LiDAR complementary coverage ..................................................... 111

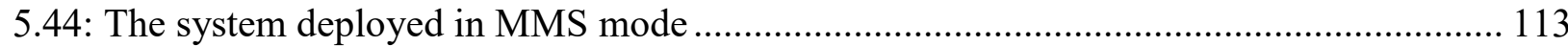

5.45: System sample reading (system horizontally mounted) ........................................... 114

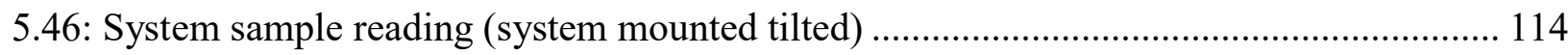

5.47: A sample Mapped area with tilted system mount...................................................... 115

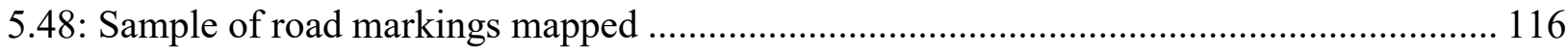

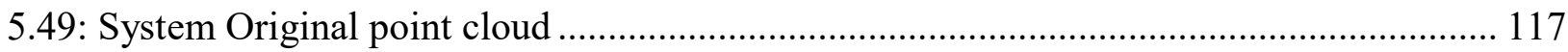




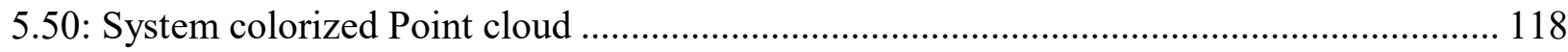

6.1: The System mounted in ULS mode on the DJI M600..................................................... 121

6.2: The System mounted in ULS mode with Hyperspectral camera........................................ 122

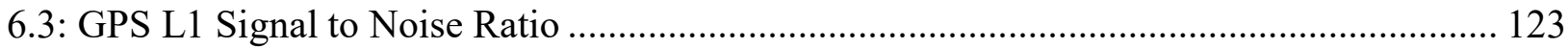

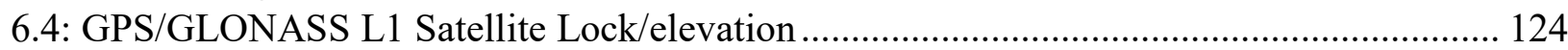

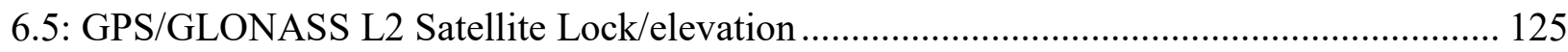

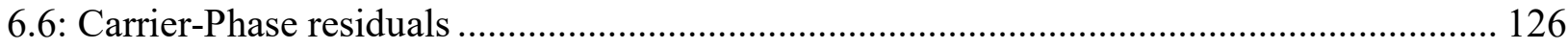

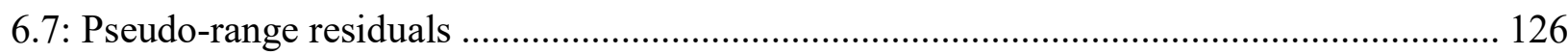

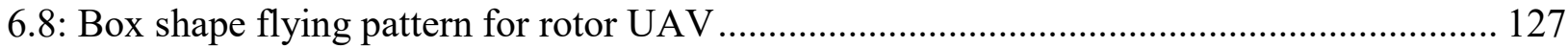

6.9: Lion's park area layout (mapped area with the system in ULS mode).............................. 129

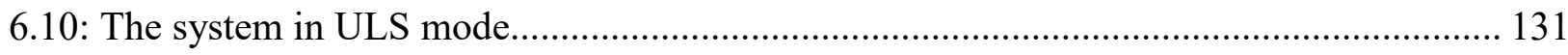

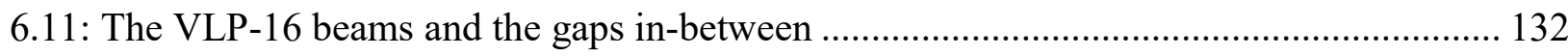

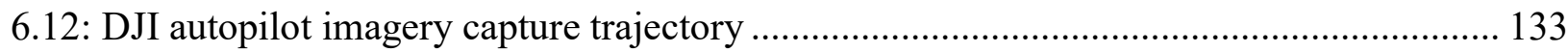

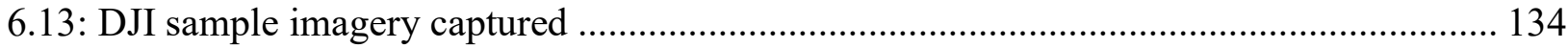

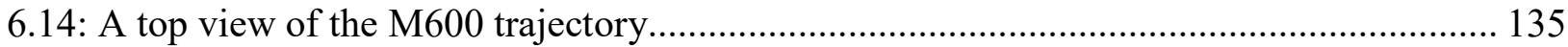

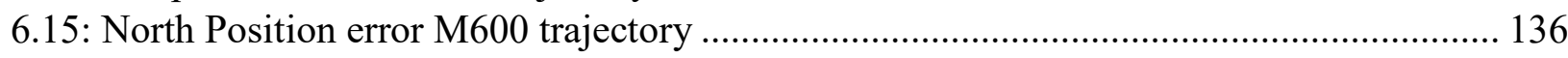

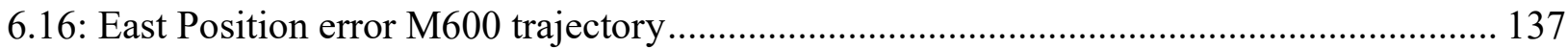

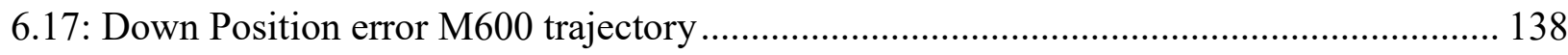

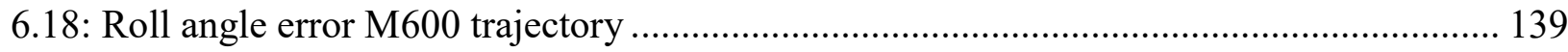

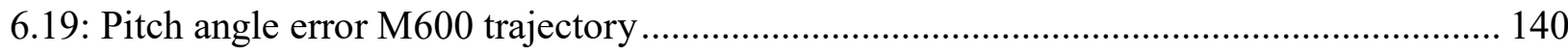

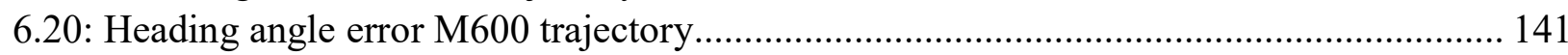

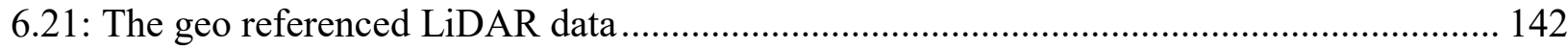

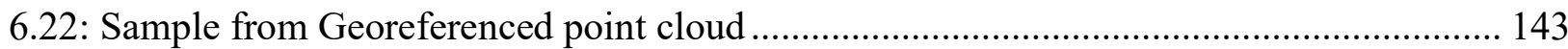

6.23: Georeferenced 3D points' distances to the mesh surface of the southern side of the park

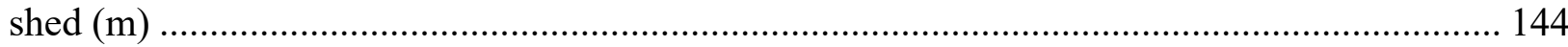

6.24: Histogram of georeferenced 3D points' distances to the mesh surface of the southern side of the park shed ............................................................................................................ 145

6.25: Georeferenced 3D points' distances to the mesh surface of the northern side of the park

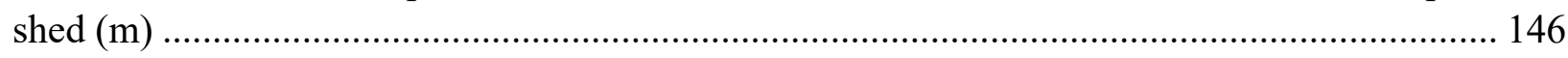
6.26: Histogram of georeferenced 3D points' distances to the mesh surface of the northern side

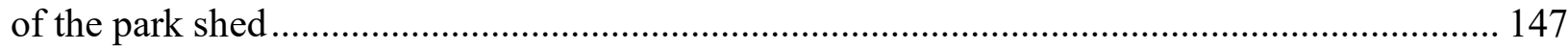

6.27: Distances to the mesh surface from ground georeferenced 3D points (m) ........................ 148

6.28: Histogram of Distances to the mesh surface from ground georeferenced 3D points ........ 149

6.29: Sample 3D point cloud from Polaris........................................................................... 150

6.30: Georeferenced 3D points' distances to the mesh surface of the northern side of the park

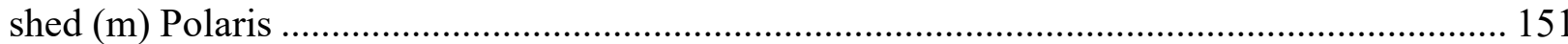

6.31: Histogram of the Georeferenced 3D points' distances to the mesh surface of the northern side of the park (Polaris) ........................................................................................................ 152

6.32: Distances to the mesh surface from ground georeferenced 3D points (Polaris)................ 153

6.33: Histogram of Distances to the mesh surface from ground georeferenced 3D points (Polaris)

154

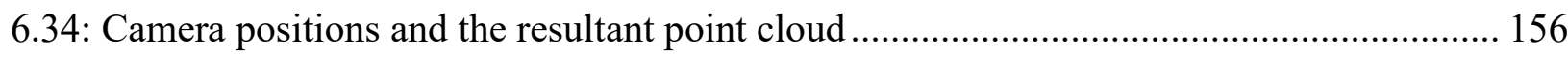

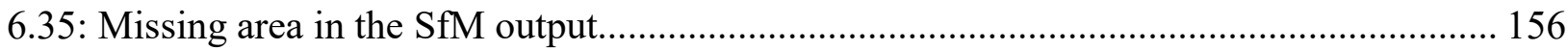




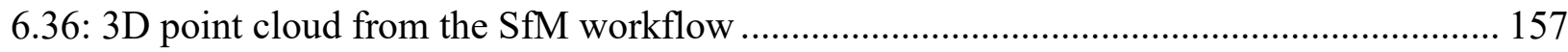

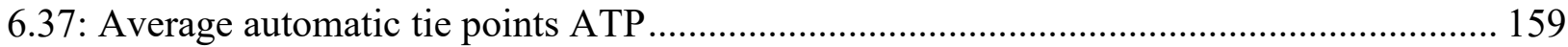

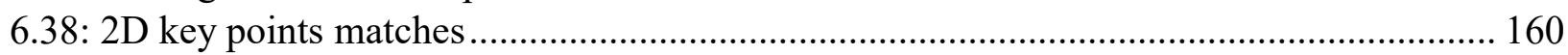

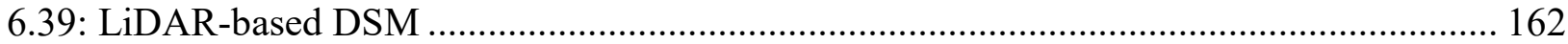

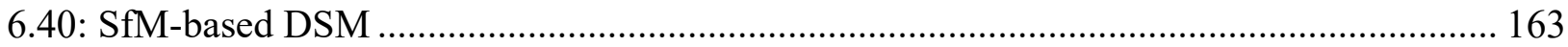

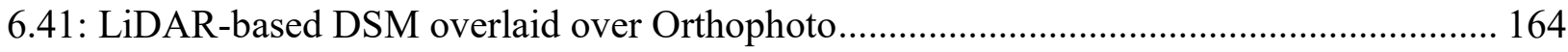

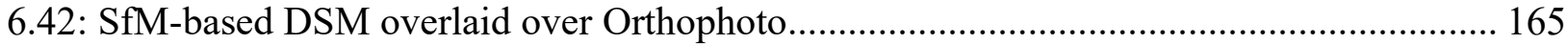




\section{List of Acronyms and Abbreviations}

$\begin{array}{ll}\text { ALS } & \text { Airborne Laser Scanning } \\ \text { APC } & \text { Antenna Phase Center } \\ \text { BBA } & \text { Bundle Block Adjustment } \\ \text { BIM } & \text { Building Information Model } \\ \text { BRIEF } & \text { Binary Robust Independent Elementary Features } \\ \text { CS } & \text { Coordinate System } \\ \text { CSF } & \text { Cloth Simulation Filtering } \\ \text { DEM } & \text { Digital Elevation Model } \\ \text { DG } & \text { Direct Georeferencing } \\ \text { DIM } & \text { Dense Image Matching } \\ \text { DSM } & \text { Digital Surface Model } \\ \text { DTM } & \text { Digital Terrain Model } \\ \text { ECEF } & \text { Earth Centered Earth Fixed } \\ \text { EMI } & \text { Electromagnetic Interference } \\ \text { FOG } & \text { Fiber Optic Gyros } \\ \text { GCPs } & \text { Ground Control Points } \\ \text { GNSS/INS } & \text { Global Navigation Satellite System /Inertial Navigation System } \\ \text { GPRMC } & \text { Global Position Required Minimum Common sentence } \\ \text { HFOV } & \text { Horizontal Field of View } \\ \text { LiDAR } & \text { Light Detection And Ranging } \\ \text { MCC } & \text { Multiscale Curvature Classification } \\ \text { MEMS } & \text { Micro Electro-Mechanical System } \\ \text { MMS } & \text { Mobile Mapping Systems } \\ \text { MoCap } & \text { Motion Capture } \\ \text { NMEA } & \text { National Marine Electronics Association } \\ \text { NTP } & \text { Network Time Protocol } \\ \text { ORB BRIEF } & \text { Oriented Fast and Rotated BRIEF } \\ \text { PMVS } & \text { Patch Multi-View Stereopsis } \\ \text { POS } & \text { Position and Orientation System } \\ \text { PPS } & \text { Pulse Per Second } \\ \text { RANSAC } & \text { RANdom SAmple Consensus } \\ \text { RLG } & \text { Ring Laser Gyros } \\ \text { RSS } & \text { Remote Sensing Sensor } \\ \text { RTK } & \text { Real Time Kinematic } \\ \text { SBET } & \text { Smoothed Best Estimate of Trajectory } \\ \text { SfM } & \text { Structure from Motion } \\ \text { SIFT } & \text { Scale Invariant Feature Transform } \\ \text { SIMD } & \text { Single Instruction to Multiple Data } \\ \text { SLAM } & \text { Simultaneous Localization and Mapping } \\ \text { SPS } & \text { Standard Positioning Service } \\ \text { STLS } & \text { Static Terrestrial Laser Scanning } \\ & \end{array}$


SURF Speed Up Robust Feature

SWaP Size, Weight, and Power

TLS Terrestrial Laser Scanning

UAV Unmanned Aerial Vehicle

VFOV Vertical Field of View

ZUPT Zero velocity Update 


\section{List of Appendices}

Appendix A: Derivation of the Developed System Uncertainty Predictive Model .................. 172 


\section{INTRODUCTION}

\subsection{Background}

Using maps is an integral part of our life, whether for navigation, geographic information systems, resources monitoring, surveying, or other pursuits and uses. Three-dimensional (3D) maps serve as an essential component in the aforementioned as well as in many new indoor and outdoor applications, such as smart cities, autonomous vehicles, asset management, virtual and augmented reality, as-built drawings, etc. Thus, the number of end-users who require 3D maps has expanded exponentially in recent years and is anticipated to expand even more in the future.

At the same time, the pace of technological progress in 3D scanning sensors is also rapidly accelerating. The two main remote sensing sensor (RSS) types typically used in the 3D mapping process are active and passive. The active sensors used in 3D mapping are mainly Light Detection And Ranging (LiDAR) sensors, while the passive sensors are mainly optical cameras. LiDAR sensors are currently considered the standard sensors used for 3D scanning. The quality of the scans obtained depends on a number of factors, including LiDAR sensor capabilities, data acquisition mode, scanned environment, target reflectivity, and the algorithms used to stitch multiple scans together. Advances in sensor technology allow a higher rate and volume of data acquisition, adding to the challenge of 3D mapping.

There are two main mapping approaches in the 3D mapping process. The first is the indirect georeferencing approach, where the relation between the mapped environment and the RSS intrinsic and extrinsic parameters is defined through the use of ground control points (GCPs). The second approach is the direct georeferencing (DG) approach, where a global navigation satellite system receiver/inertial navigation system (GNSS/INS) sensor is used to provide the position and orientation of the RSS. GNSS/INS-provided measurements enable the building of a relation between the RSS and the environment being mapped. Since the second approach can be utilized without the need of GCPs and is better suited to the irregular nature of the LiDAR data (reflective targets are needed if the first approach is to be used with LiDAR), DG is considered more practical and time-efficient, especially for mapping large areas. The choice of the second approach could also be attributed to recent technological advances in GNSS/INS sensors.

In the DG approach, the mapping system consists mainly of three components: 1) the active and/or passive RSS to sense the environment; 2) the GNSS/INS system to allow the DG; and 3) the data management unit for control, power and data acquisition storage. The mapping system is mounted on a platform which defines the acquisition mode. Single/multi-LiDAR scanners are used in LiDAR-based mapping systems. The acquisition mode may be either Terrestrial Laser Scanning (TLS) or Airborne Laser Scanning (ALS). More specifically, TLS can be categorized according to the platform onto which the mapping system is mounted, as being either a stationary STLS (usually a tripod) or Mobile Mapping Systems (MMS) (a kinematic platform, typically a vehicle or on an operator backpack); the ALS platforms may be either a manned aircraft or an Unmanned Aerial Vehicle (UAV). 
The mapping system characteristics differ significantly according to the intended acquisition platform used. For instance, the constraints regarding the mapping system Size, Weight, and Power (SWaP) consumption are much more stringent with a UAV-based mapping system than those for a STLS. There has been a paradigm transferral in the mapping process since the introduction of MMS almost two decades ago, as it allowed for rapid data acquisition over large mapping areas that is time- and labour-prohibitive with traditional TLS.

ALS is a proven aerial mapping technology that has been deployed for decades, typically using manned airplanes. The ability of multiple LiDAR return measurements proved beneficial in forestry applications and in the Digital Surface Model (DSM) and Digital Terrain Model (DTM) generation. ALS tends to be very expensive with high operational costs and extensive pre-mission planning and requirements. The recent technological advancement in this sector was the introduction of the Teledyne multispectral LiDAR that operates in three bands. However, as this version is still quite costly and also has extensive pre-deployment requirements, it suits few enduser segments and is more suited to specific types of applications such as bathymetry or land-water delineation. In contrast, recent advances in UAV technology, including its low cost as a mapping platform, relative ease of deployment, high maneuverability, ability to be deployed in human-risky environments and appeal to many end-user segments, could explain the rapid growth in the UAV market. A great deal of research has been geared towards the development of UAV photogrammetry-based mapping, despite LiDAR-based UAV mapping being a relatively new research area.

Another mapping sector that has experienced recent rapid growth is indoor mapping. For instance, the required data for the Building Information Model (BIM) and the new end-user segment that it represents has allowed for the introduction of a number of indoor mapping systems. Some of these systems are deployed as a handheld or backpack mapping systems. Typically, the mapping utilizes the Simultaneous Localization and Mapping (SLAM) approach, whether visual-SLAM or LiDARbased SLAM. However, these mapping systems are intended for indoor environments, so they are not well-suited for outdoor mapping.

Additionally, advances in computer vision algorithms are positively affecting the 3D mapping process. One workflow for 3D mapping from optical imagery is the Structure from Motion (SfM) technique. The recent advances in SfM photogrammetric technique, coupled with its relatively low cost compared to LiDAR scanners and its ability to produce dense point clouds and comparable results, prove it to be a viable alternative. Nevertheless, one shortcoming of the SfM is its relatively low performance in poorly textured areas and places with repetitive patterns, as well as its dependency on lighting conditions and imagery-capturing geometry to produce reliable results.

Digital Surface Model (DSM) is a crucial component in many fields, such as hydrological studies, watershed analysis, and orthophoto generation. With the recent advances in SfM workflow and UAV-photogrammetry, a number of studies were conducted utilizing UAV-SfM in the DSM generation. Nevertheless, its reliable application is dependent on the aforementioned conditions, as it is considered error-prone in poorly textured areas. The integration of SfM and LiDAR data will complement the strengths of each methodology while mitigating their drawbacks. 
Despite the increased demand for 3D mapping as more end-user segments require 3D maps for their respective domain applications, traditional mapping systems remain relatively costprohibitive. In addition, the technical resources required for the mobilization of these mapping systems adds to their impracticality to wider end-user groups. This is further complicated by the fact that a mapping system is usually developed to suit one mode of operation, whether STLS, MMS, or ALS. Moreover, despite the preference for UAV-SfM in DSM generation, its shortcoming in poorly textured areas and the adverse effect of vegetation cover need to be addressed. Thus, the primary focus of this research is the development of a generic framework for a LiDAR-based 3D mapping system that follows a plug-n-go concept with platform-switching capabilities. The research will also pursue the accurate 3D reconstruction of LiDAR data through a smart integration workflow at the hardware and software levels, along with a new mapping scheme.

\subsection{Motivation}

In today's highly integrated technological environment, more and more applications require 3D mapping products that traditional mapping systems are unable to deliver. Applications include precision agriculture, powerline mapping and inspection, pipeline mapping and inspection, forensic scene reconstruction, corridor mapping, mine monitoring, archeological site documentation, and DSM generation for small areas. The recent technology advances in mapping system hardware components and processing workflows provide an opportunity to address the non-traditional demand on 3D mapping from diverse end-users if the 3D mapping system components are effectively integrated on the hardware (HW) and software (SW) levels. Finding the right balance between accuracy and mapping system costs further adds to the 3D mapping challenge. The utilization of low-end system components will meet the cost constraints but will add to the complexity of the research effort and innovative nature required to maximize the attainable accuracy.

Unmanned aerial vehicles (UAVs) as a mapping platform have gained momentum in recent years to bridge the gap between the unfeasibility of using manned ALS for mapping small areas and the intensive time and labour requirements associated with using STLS. Research using UAV photogrammetry-based equipment for 3D mapping has accelerated rapidly over the past few years due to the introduction of the SfM workflow and technology advances in optical cameras. Nevertheless, few research efforts were directed towards LiDAR-based UAV mapping, as it is a new area of research.

Despite the latest technology advances (especially for DG sensors and LiDAR scanners, including their increased miniaturization), MMS is still generally bulky, expensive, difficult to deploy, and requires a higher level of expertise from the end-user. Major investment and operational costs, unease of deployment and the required level of expertise hinder the current MMS usage for many user segments that may need this technology. A crosscutting LiDAR-based 3D mapping system will address the needs of diverse end-users and meet the ever-increasing demand for 3D mapping that can be applied to a plethora of applications. The advances in computer vision algorithms (more specifically, SfM) provides a means to augment the interpretation of the mapped environment when adequately fused with LiDAR-based 3D mapping. 


\subsection{Problem Statement and Research Challenges}

An accurate cost-effective 3D mapping pipeline is a research challenge. The existing 3D mapping systems are out of reach of many end-users, as they are considered cost-prohibitive. In addition, the level of technical expertise required for the system mobilization hinders the system utilization by many end-users. Thus, meeting diverse end-users needs and the emerging new applications that requires 3D mapping with traditional 3D mapping systems is uncertain.

The utilization of the state-of-the-art compact LiDAR sensors, originally developed for the autonomous vehicles industry, as the RSS in 3D mapping systems is a new research area. A number of research challenges need to be addressed in order to meet the diverse ever-increasing need for 3D mapping with novel mapping systems. The efficient fusion of multimodal sensor data is yet another research challenge that needs to be resolved. The following research problems are of significant interest to the research community regarding non-traditional 3D mapping systems and their data integration algorithms.

i) The optimization of the mapping system sensors' selection and placement: the SWaP and placement of the mapping system components need to be prudently considered in order to allow optimal mobilization independent of the platform used, along with the proper handling of the Electro-Magnetic Interference (EMI) of system components.

ii) The precise signal synchronization of the different mapping system components; sensors synchronization is a major concern, as it is critical for ensuring the accurate outcome of the mapping system.

iii) Reliable system control and time-efficient data processing: the reliability and ease of controlling the mapping system in different data acquisition modes adds to its robustness and ease of deployment, so this is another important aspect that needs to be addressed. The time-efficient data processing is vital for minimizing the turnaround time of the 3D mapping outcome, especially for mapping large areas.

iv) A complete rigorous uncertainty predictive model to predict the uncertainty in the $3 \mathrm{D}$ georeferenced point cloud is essential to quantify the relation between the precision of the system components and the system data output uncertainty.

v) Enhancing the visual interpretation of the LiDAR data: the color encoding of LiDAR with optical imagery data serves to enhance visual interpretation, thus meeting wider user-base requirements.

vi) Enhancing the UAV-SfM DSM generation: research efforts need to be exerted to enhance the UAV-SfM 3D mapping and DSM generation workflow, especially in poorly-textured areas, to extend its applicability, serve as a new workflow for the fusion of LiDAR point cloud color encoding, and enhance its visualization. 


\subsection{Research Objectives}

As discussed in the previous sections, to address the ever-increasing 3D mapping demand from heterogeneous end-user types and to suit diverse applications, a new 3D mapping pipeline is essential. Accordingly, the objective of this dissertation is to develop a novel, accurate, costeffective 3D mapping framework that entails a unique LiDAR-based mapping system.

The design criteria for the LiDAR-based mapping system can be summarized as being i) costefficient with ii) survey-grade accuracy iii) platform-independent iv) plug-n-go capability, in addition to being v) versatile and modular, vi) flexible to be adopted indoor and vii) easily modifiable concerning the components used to easily integrate any upgraded sensors.

The steps to fulfill the design criteria can be summarized as i) starting with the optimization of the multimodal sensors selection, ii) relative placements of the system components, iii) synchronization and the iv) sensors integration on the hardware HW and software SW levels

Thus, this research main objectives are as follows:

- The design, development and implementation of a LiDAR-based DG generic 3D mapping system that can smoothly be applied in stationary, mobile and UAV-based mapping modes.

- The development of a new mapping scheme that enables platform-independent deployment ability

\subsection{Dissertation Outline}

This dissertation is organized as follows:

- Chapter 2 provides a literature review of the existing pertinent literature, starting with the LiDAR data acquisition principle, various types of LiDAR sensors, 3D mapping systems in different data acquisition modes, and system orientation optimization. This is followed by the SfM algorithm and DSM generation, along with more in-depth coverage of UAVbased DSM generation. Finally, LiDAR and optical imagery data fusion is reviewed.

- Chapter 3 introduces the proposed strategy for the new mapping scheme and the development of the LiDAR-based mapping system architecture.

- Chapter 4 presents the proposed mapping system's various modes of operation and the uncertainty predictive model.

- In Chapter 5, the mapping system realization in stationary and mobile modes of operation and the point cloud colorization are discussed. 
- The mapping system realization in the UAV mode of operation, along with SfM and DSM generation are investigated in Chapter 6.

- Finally, in Chapter 7, a summary of the dissertation and its major findings is provided, together with conclusions, key contributions and recommendations for future research work. 


\section{LITERATURE REVIEW}

\subsection{Overview}

As discussed in Chapter 1, the main objective of this research is the development of a novel, accurate, cost-effective 3D mapping framework utilizing an in-house-developed LiDAR-based mapping system, which can be operated in the three traditional mapping modes. The research will also test the ability of generating DSM as a derived product from the system output and comparing that to the SfM-based DSM generation workflow. The review of the existing literature pertaining to 3D mapping systems, the SfM algorithm, DSM generation and LiDAR-optical imagery data fusion is the focus of this chapter. The first part will cover the LiDAR data acquisition principle, various contemporary types of LiDAR sensors, 3D mapping systems in different data acquisition modes, and system orientation optimization. The second part of the chapter highlights the SfM algorithm and DSM generation, and provides a more in-depth coverage of UAV-based DSM generation and LiDAR and optical imagery data fusion.

\subsection{LiDAR Data Generation}

A LiDAR scanner is an active Remote Sensing Sensor (RSS) like radar and sonar, but it uses light as the source of target illumination. The LiDAR unit emits a pulsed light beam or a continuous wave that hits the target area and reflects back. The precise measurement of the Range (R) follows one of two main methods [1]. The first method is the accurate measurement of the Time Of Flight (TOF), which is the time interval that has elapsed between the emission of a short but intense light pulse by the sensor and its return after being reflected from an object. From this, R can be calculated, as illustrated in Equation (2.1). The precision of the time measurement determines the range measurement precision, as defined in Equation (2.2).

$$
R=v \cdot t / 2
$$

Where

$R$ is the range

$v$ is the speed of the electromagnetic radiation(very accurately known)

$t$ is the time interval measured

And the range precision can be determined as follows

$$
\Delta R=\Delta v \cdot t / 2+v \cdot \Delta t / 2
$$

Where

$\Delta R$ is the range precision

$\Delta v$ is the speed of the electromagnetic radiation precision

$\Delta t$ is the time interval 
In the second method, the sensor emits a continuous beam of laser radiation instead of a pulse. The accurate measurement of the phase difference between the emitted wave signal and the signal received by the sensor after being reflected from the target determines the slant range to the object, as shown in Equation (2.3). The first method is more common.

$$
R=(M \lambda+\Delta \lambda) / 2
$$

Where
$R$ is the range
$M$ is the integer number of wavelengths $(\lambda)$
$\Delta \lambda$ is the fractional part of the wavelength

The laser unit steers the light beam through a mirror or a prism mechanism to cover the vertical direction which, when coupled with a controlled and measured motion in the azimuth direction (in the STLS case), a sequence of profiles around the vertical axis of the laser unit is measured and a $3 \mathrm{D}$ point cloud of the area around the laser unit is generated. However, if the laser unit is mounted on a moving platform, the controlled and measured motion in the azimuth direction may be substituted by the platform movement, as it covers the third dimension.

Another factor that affects the 3D point cloud generated is beam divergence [1]. Beam divergence is an angular measure that relates the increase in the beam radius or diameter to the distance it travels after being emitted by the laser unit. Although the light beam is collimated when emitted from the laser unit, the beam will diverge as it propagates, and the divergence will affect the footprint that is measured by the beam. Thus, the measured distance will represent a wider area on the target and, in turn, will decrease the specificity of the measured distance, as it will miss any position variation within the footprint. The effect is further stressed with long-range sensors, such as those used in ALS. This explains the narrow beam divergence in the sensors used for ALS (which is typically $0.5 \mathrm{mrad}$ or less), as this translates to a laser footprint of $50 \mathrm{~cm}$ or less at a flying height of $1000 \mathrm{~m}$.

In addition, laser scanners can measure the amount of energy that has been reflected from the target after being illuminated with the scanner emitted pulse. The amount of reflected energy from each point measured constitutes the intensity as measured by the sensor. This measurement can prove valuable in a number of applications [2]. The intensity measured depends on the target reflectivity, which affects the amount of energy reflected that can be detected by the sensor [3]. As the target reflectivity decreases, the amount of reflected energy diminishes, thus weakening the signal returned to the sensor and deeming the target undetectable. The range and incidence angle to the target also affects the measured intensity [4].

Unlike 2D laser scanners that depend on the platform movement to cover the third dimension, a new type of laser scanners was introduced recently to meet autonomous vehicle industry requirements. These new LiDAR scanners are known as spinning multi-beam LiDAR scanners. Instead of having a single beam laser and a rotating mirror or prism, the new sensors have multiple beams with pairs of an emitter and a receiver for each beam. Each beam is oriented at a fixed 
vertical angle from the sensor origin. The multiple beams spin mechanically around a spinning axis with up to a frequency of $20 \mathrm{~Hz}$ with Velodyne LiDAR sensors covering a $360^{\circ}$ Horizontal Field of View (HFOV) [5]. This builds a fast and rich 3D point cloud of the vehicle's environment, enhancing its 3D perception. Velodyne laser VLP-32c and Quanergy M8 [6] are two examples of these sensors, as shown in Fig. 2.1.

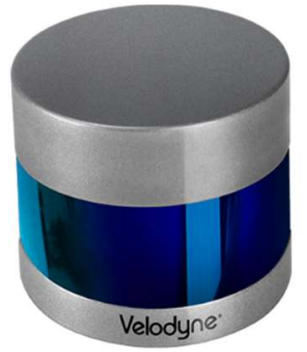

(a)

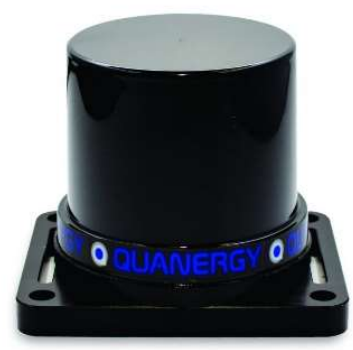

(b)

Fig 2.1: Examples of spinning multi-beam laser sensors,

a) The velodyne VLP-32c and b) Quanergy M8

Another laser scanning technology is the solid-state flash LiDAR. Unlike the previously discussed sensor data measurement mechanisms, this new technology illuminates large areas simultaneously and measures the reflected energy on a photonic phased array [7]. The measuring mechanism is an analogous resemblance of the digital camera complementary metal-oxidesemiconductor(CMOS) sensor. The sensor does not have any moving parts and the miniaturization allows on-chip lasers. Velodyne velarray, Quanergy S3 and Leddartech M16 are examples of these sensors, as shown in Fig. 2.2.

It is worth noting that the current rapid increase in laser sensor technology is being driven by the automotive autonomous vehicle application domain. The solid state LiDAR sensors are a very promising technology but are not yet well-suited for 3D mapping applications, as they are specifically designed for vehicle environment grid occupancy detection and collision avoidance [8], [9]. As the technology advances, however, it is anticipated that the solid-state LiDAR technology will have a positively disruptive effect on the 3D mapping field.

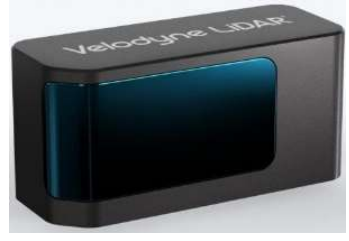

(a)

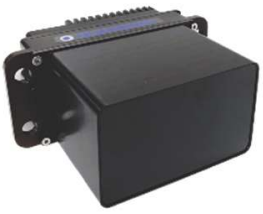

(b)

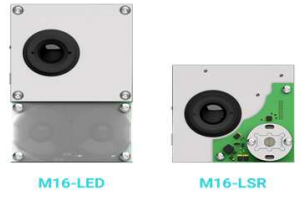

(c)

Fig 2.2: Examples of solid state LiDAR,
a) The velodyne Velarray
b) Quanergy S3 and (c) Leddartech M16-LSR 


\subsection{LiDAR-based Mapping Systems}

The introduction of MMS has dramatically altered the 3D mapping process since its introduction almost two decades ago. The rapid mobile terrestrial-based data acquisition and mapping of large areas in a short time has been considered a major breakthrough in the mapping field. One of the earliest research efforts in the development of a MMS is the work of the center of mapping at Ohio State University, where the MMS GPS-VAN was developed by integrating a code-only GPS receiver, passive sensors (CCD and video cameras), and dead-reckoning sensors [10]. LiDARbased mapping systems followed in the late 2000s and early 2010s. Large companies like TOPCON and Trimble along with medium-sized companies like OPTECH, RIEGL, MDL, SITECO, 3D LASER MAPPING and IGI are well-established suppliers of MMS for the mapping community $[10,11]$. Examples of these high-end commercially available MMS are the OPTECH Lynx system and the Trimble MX8, shown in Fig. 2.3. A detailed comparison between these two MMS and other high-end mapping systems can be found in [10]. Although these mapping systems provide a very dense accurate point cloud, they are still bulky and expensive and require skilled operators along with a lengthy time for data processing. These issues make them difficult to obtain for a wide spectrum of end-users that may need the technology.

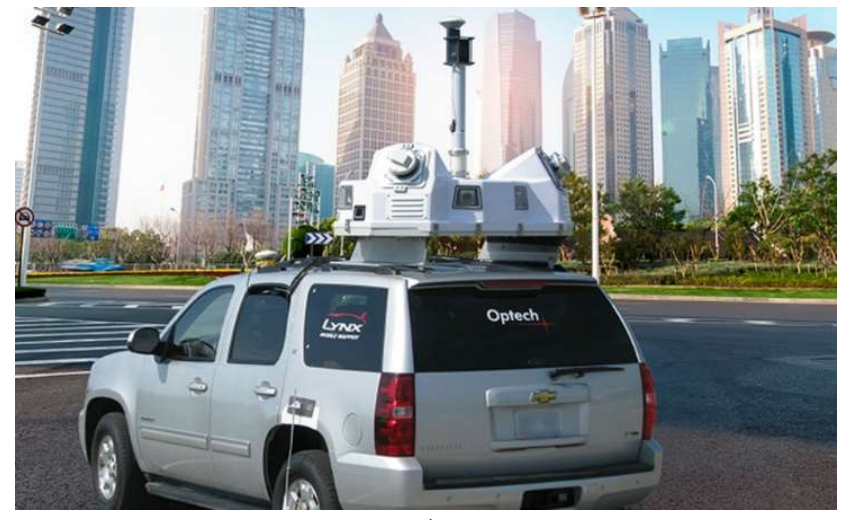

a)

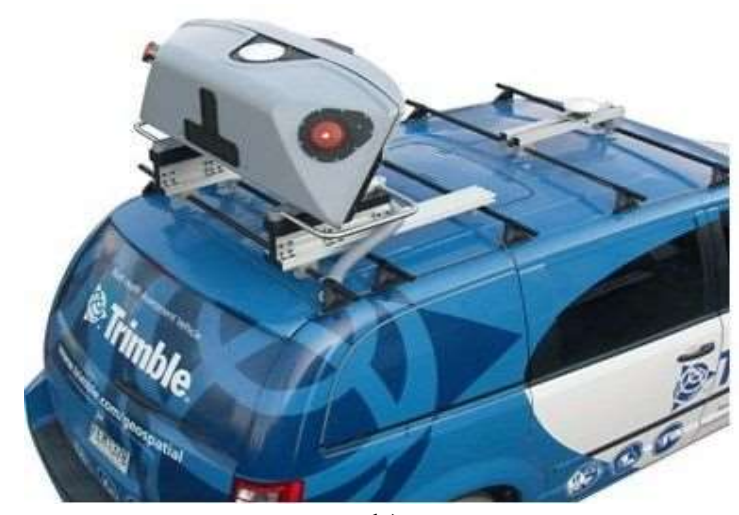

b)

Fig 2.3: Examples of Commercial MMS,

a) Optech Lynx and b) Trimble MX8

Some of the innovative MMS that were recently developed by commercial companies are the Optech Maverick, Trimble Mx2, and Topcon IP-S3 (Fig. 2.4). These three MMS have a smaller form-factor, and two of them (the Maverick and the IP-S3) use one of the new spinning multibeam LiDAR SMbL scanners. Mapping-grade MMS normally achieves sub-meter accuracy and costs $\sim \$ 400 \mathrm{k}$ US, while survey-grade MMS achieves cm-level accuracy and typically costs $\sim \$ 1$ million US [11]. Thus, these MMS are considered cost-prohibitive for many end-users, along with the difficulty of special considerations that are required for mounting. 


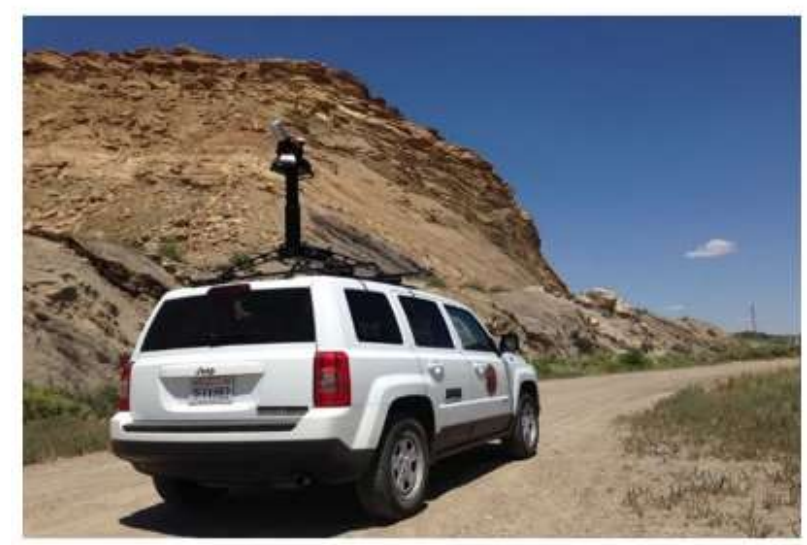

a)

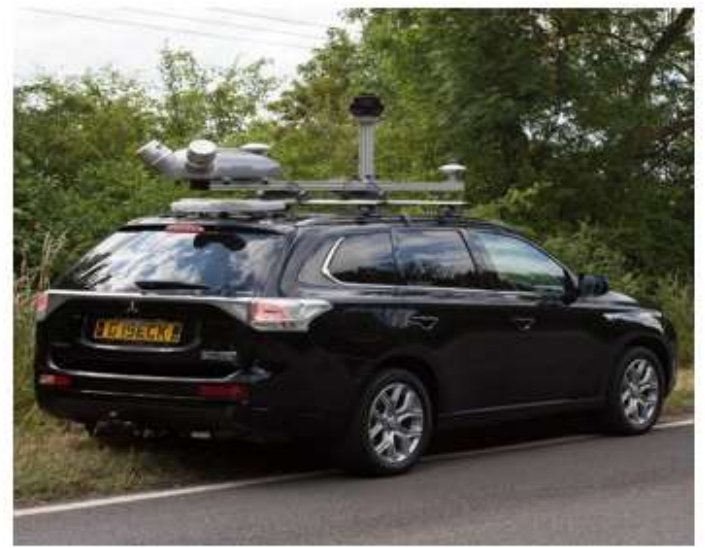

b)

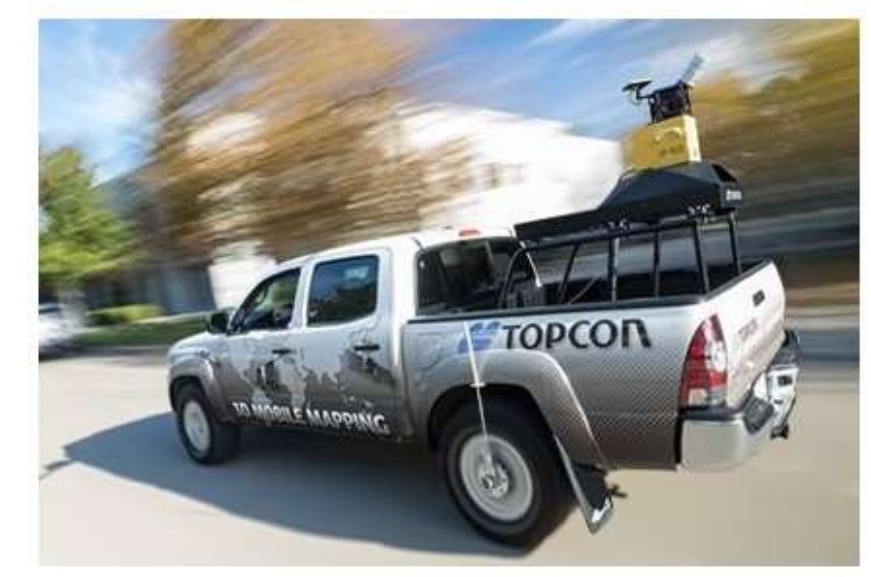

c)

Fig 2.4: Examples of recently developed commercial MMS,
a) Optech maverick
b) Trimble MX2 and c) Topcon IP-S3

The technological advances in LiDAR sensors coupled with the advances in the GNSSS/INS sensors prompted a number of recent research efforts to exploit the development of purpose-built mapping systems that utilize the new spinning multi-beam LiDAR SMbL scanners. This is a very new area of research, as these sensors were released only a few years ago. As discussed previously, a mapping system can be categorized according to the platform on which it is deployed. A few studies capitalized on the usage of the SMbL in MMS. Julge et al. [12] utilized a VLP-16 LiDAR sensor and an unspecified INS system to build an MMS, reporting errors of up to $5 \mathrm{~m}$ while cornering and swerving. Sairam et al. [13] used the HDL-32E LiDAR scanner and Geodetics GeoiNav INS system for road asset management. Julge et al. [14] employed a VLP-16 and an SBG Ellipse-D INS system, reporting accuracies of $15 \mathrm{~cm}$. These research efforts, however, only address the design and development requirements for systems to be utilized in the MMS mode, without taking into consideration the design and development challenges that need to be addressed 
in order to offer the crosscutting ability of the system to be utilized in all three mapping modes (i.e., stationary, mobile and ULS).

The development of UAV-based LiDAR ULS constitutes most of the new research efforts for the development of purpose-built mapping systems that utilize the new spinning multi-beam LiDAR SMbL scanners. This could be attributed to the compact size and light weight of the new spinning multi-beam LiDAR scanners, which prove advantageous, as they satisfy the stringent SWaP requirements for UAV deployment. UAV originated for military applications [15] and then quickly gained utilization in civilian applications almost a decade ago. UAV can be categorized according to different parameters. The most common one used is the UAV ready-to-fly system weight, as this is the weight that is compared to the aviation regulations. The different UAV platform classes are enumerated in Table 2.1. Note that the UAV platforms used in civilian mapping applications generally belong to the micro and mini classes [16].

Table 2.1: UAV classes, adopted after Rehak [16]

\begin{tabular}{|l|c|c|c|c|}
\hline & Mass $(\mathrm{kg})$ & Range $(\mathrm{km})$ & Flight Alt. $(\mathrm{m})$ & Endurance $(\mathrm{h})$ \\
\hline Micro & $<5$ & $<10$ & 250 & 1 \\
\hline Mini & $<20-25$ & $<10$ & 500 & $<2$ \\
\hline Close range & $25-150$ & $10-30$ & 3000 & $2-4$ \\
\hline Short range & $50-250$ & $30-70$ & 3000 & $3-6$ \\
\hline Medium-range & $150-500$ & $70-200$ & 5000 & $6-10$ \\
\hline
\end{tabular}

UAVs have a number of different types and sizes, with the selection of a UAV as a mapping platform depending on several factors. The key ones that need to be considered are endurance, payload capacity, type of equipment to be deployed, take-off and landing method, cost, and ease of use. The endurance will affect how long and how far the system can be deployed, consequently defining the area that can mapped. UAVs suitable for small-area mapping and close-range photogrammetry are different from those required to suit aerial mapping of large areas. Payload capacity is another defining factor in the selection of a UAV, as this will limit the type of sensors that can be deployed on the UAV. It also defines the total system weight, which has to meet the regulations. Additionally, vibration performance and system interference will affect the type of equipment that can be used. According to UAV type, special considerations may be required for take-off and landing, which affects the UAV selection process. Cost also plays a role. Lastly, how easy the UAV is to operate and whether or not manual flying of the UAV is required likewise affects the selection process [16].

The propulsion mechanism used for UAV operation can be used to classify UAVs. Fixed wings, multirotor and helicopters are the main airborne-based platforms used for ALS. Table 2.2 provides a comparison between the advantages and drawbacks of each type, along with the relevant applications. Some UAV examples are shown in Fig. 2.5. 
Table 2.2: Comparison of different UAV types, adopted after Chapman [17]

\begin{tabular}{|c|c|c|c|c|c|}
\hline & Advantages & Drawbacks & Application & Price & Example \\
\hline Multirotor & $\begin{array}{l}\text {-Accessibility } \\
\text {-Ease of use } \\
\text {-Good camera } \\
\text { control } \\
\text {-Can be } \\
\text { operated in } \\
\text { confined spaces }\end{array}$ & $\begin{array}{l}\text {-Short flight } \\
\text { times } \\
\text { - small } \\
\text { payload } \\
\text { capacity }\end{array}$ & $\begin{array}{l}\text {-Aerial } \\
\text { photography } \\
\text { and video } \\
\text { aerial } \\
\text { inspection } \\
\text { (recently } \\
\text { LiDAR as } \\
\text { well) }\end{array}$ & $\begin{array}{l}5 \mathrm{~K}-65 \mathrm{~K} \\
\mathrm{CAD} \$ \text { for } \\
\text { pro UAVs }\end{array}$ & $\begin{array}{l}\text {-DJI } \\
\text { M600 }\end{array}$ \\
\hline Fixed wing & $\begin{array}{l}\text {-Long flight } \\
\text { times } \\
\text {-Large area } \\
\text { coverage } \\
\text {-Fast flight } \\
\text { speed }\end{array}$ & $\begin{array}{l}\text {-A lot of } \\
\text { space } \\
\text { required for } \\
\text { take-off and } \\
\text { landing } \\
\text {-Harder to } \\
\text { fly, more } \\
\text { experience } \\
\text { needed } \\
\text {-Expensive }\end{array}$ & $\begin{array}{l}\text { Aerial } \\
\text { mapping, } \\
\text { Pipeline and } \\
\text { Power line } \\
\text { inspection }\end{array}$ & $\begin{array}{l}25 \mathrm{~K}-120 \mathrm{~K} \\
\text { CAD\$ for } \\
\text { pro UAVs }\end{array}$ & $\begin{array}{l}\text {-Sirius } \\
\text { Pro }\end{array}$ \\
\hline $\begin{array}{l}\text { Helicopter(more } \\
\text { used as a } \\
\text { manned } \\
\text { aircraft) }\end{array}$ & $\begin{array}{l}\text {-Long } \\
\text { endurance } \\
\text {-Heavier } \\
\text { payload } \\
\text { capacity }\end{array}$ & $\begin{array}{l}\text { - Harder to } \\
\text { fly, more } \\
\text { training } \\
\text { needed } \\
\text {-Expensive }\end{array}$ & $\begin{array}{l}\text { Aerial } \\
\text { Mapping }\end{array}$ & $\begin{array}{l}25 \mathrm{~K}-300 \mathrm{~K} \\
\mathrm{CAD} \$\end{array}$ & $\begin{array}{l}\text { Single- } \\
\text { rotor } \\
\text { heli }\end{array}$ \\
\hline
\end{tabular}




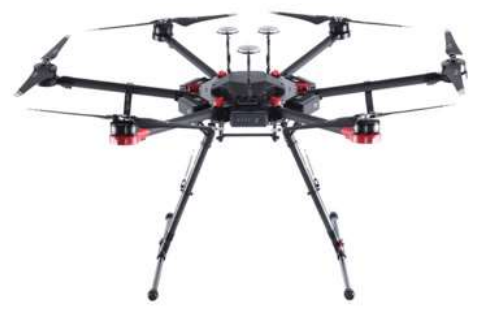

a)

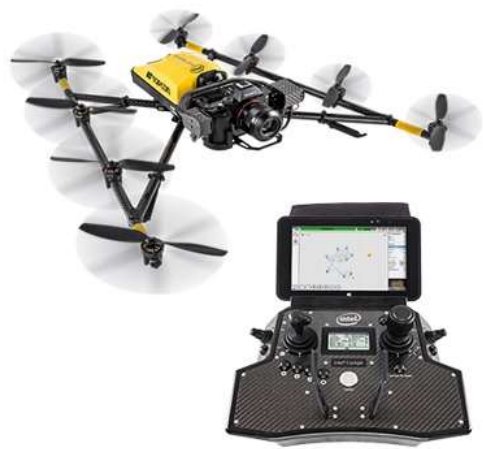

b)

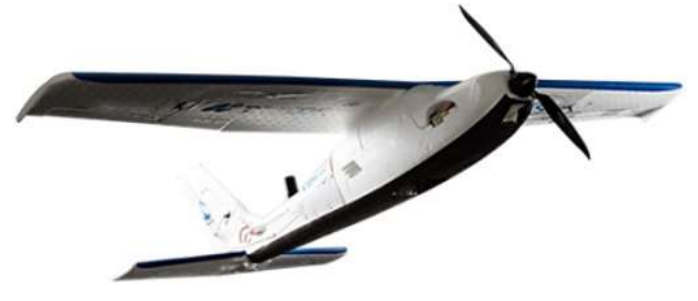

c)

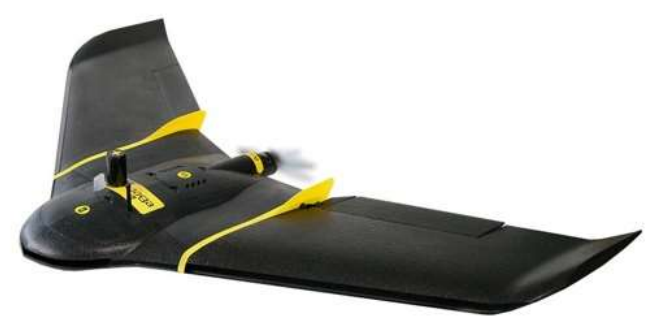

d)

Fig 2.5: Examples of different UAVs types, Multirotor a) DJI M600, b) Topcon Falcon and Fixed wing c) Topcon Sirius pro, d) ebee sensefly

A handful of research efforts are geared towards ULS LiDAR-based mapping systems. Li et al. $[18,19]$ integrated a VLP-16 LiDAR scanner along with Xsens MTI-300 INS on a DJI M600, utilizing the system in forest mapping applications, while Roca el al. used a 2D LiDAR scanner (a Hokuyo UTM-30LX along with a decoupled IMU and GNSS sensors [Spatial IMU and the Trimble BD920, respectively]). Christiansen et al. [20] used a VLP-16 scanner along with a vectornav INS deployed on a DJI Matrice 100 platform for agriculture surveying. Teng et al. [21] developed a UAV-based LiDAR mapping system, without specifying the component types used, and demonstrated its usage in power line inspection and sag determination.

A number of commercial state-of-the-art UAV-ready LiDAR systems have also been developed recently. For example, Phoenix LiDAR systems developed the Alpha AL3-32, integrating the HDL-32E and a fiber-optic INS and giving a position accuracy of $1 \mathrm{~cm}+1 \mathrm{ppm}$ RMS horizontal and attitude and heading RMS errors of $0.019 / 0.074^{\circ}$ [22]. This system is shown in Fig. 2.6. In addition, Routescene developed a compact UAV LiDAR-based system known as the Lidarpod, which encompasses a HDL-32E scanner along with an RTK GNSS/INS and a radio telemetry to send the RTK corrections from the provided ground base station. The stated absolute positional accuracy is given as $4 \mathrm{~cm}$ at a $20 \mathrm{~m}$ range [23]. Figure 2.7 illustrates the system. 

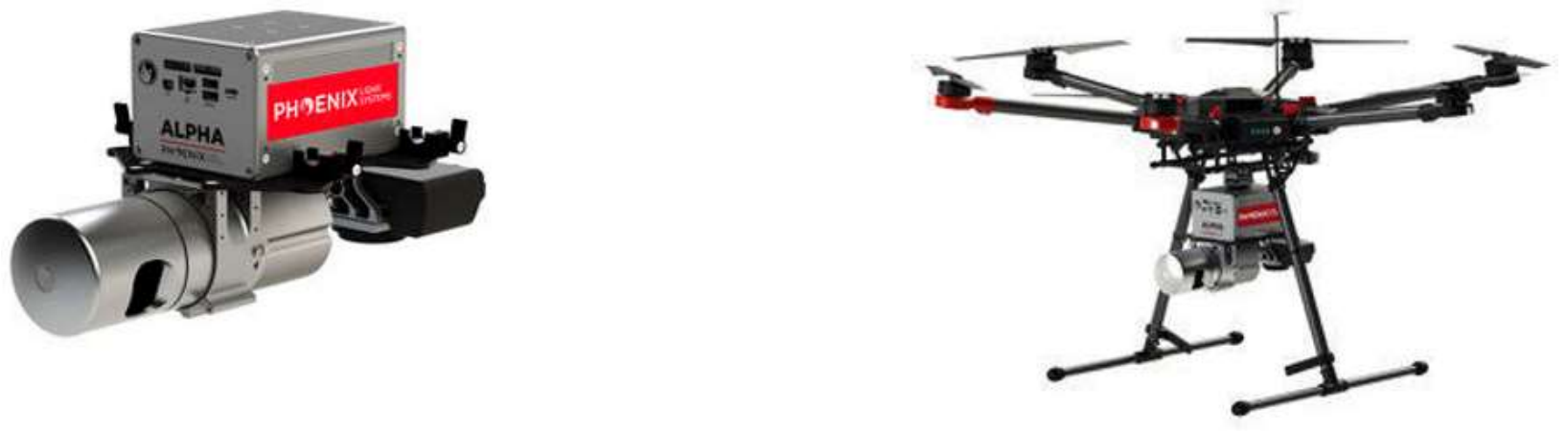

Fig 2.6: Phoenix AlphaAL3-32
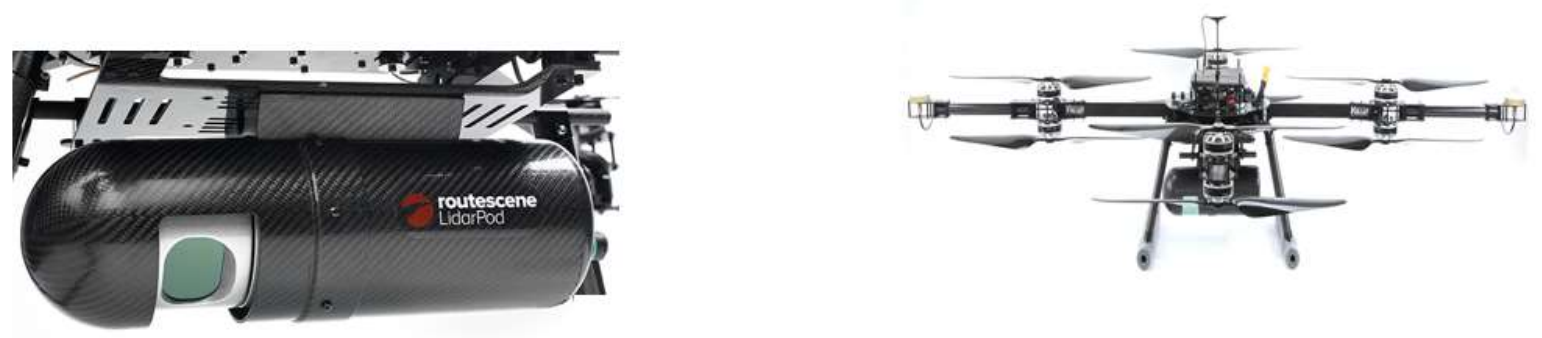

Fig 2.7: Routescene Lidarpod 
The Yellowscan surveyor is yet another UAV-ready LiDAR-based lightweight mapping system, thus adding to the suitability of its usage on UAVs with small payload capacity. The Yellowscan surveyor system employs a VLP-16 scanner and the APX-15 GNSS/INS system, giving a positional accuracy of $5 \mathrm{~cm}(1 \sigma$ at $50 \mathrm{~m})$ nadir [24], as shown in Fig. 2.8. The Rigel VUX-240 is a new LiDAR-based mapping system offered by Riegl which entails a high-end Riegl continuous waveform LiDAR scanner with a constantly rotating polygon mirror and a measurement rate of up to 1.5 million measurement/sec. It gives a reported accuracy of $2 \mathrm{~cm}(1 \sigma$ at $150 \mathrm{~m})$ [25] and is shown in Fig. 2.9.

One advantage of purpose-built mapping systems over commercially available ones is the ease of applying modification and upgrading system components as needed. Moreover, purpose-built mapping systems tend to have substantial cost reductions compared to commercial ones. However, it is worth noting that both the few research efforts and the commercially available aforementioned mapping systems were initially designed to serve one mode of operation (ULS), and so lack the flexibility and ease of deployment in the other two mapping modes (MMS and stationary).
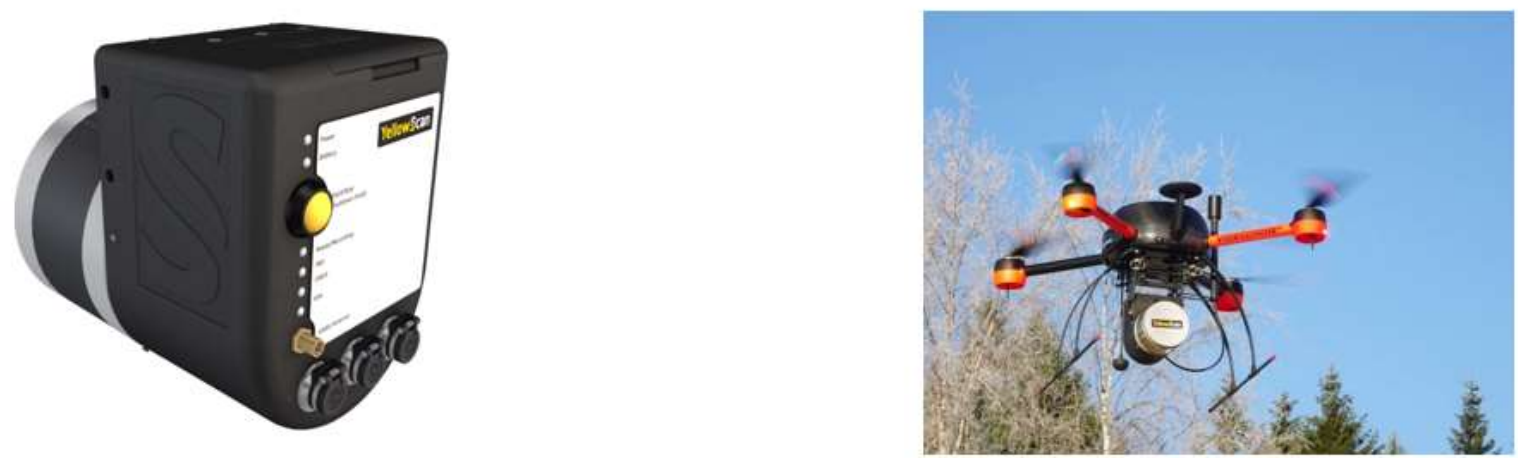

Fig 2.8: Yellowscan surveyor
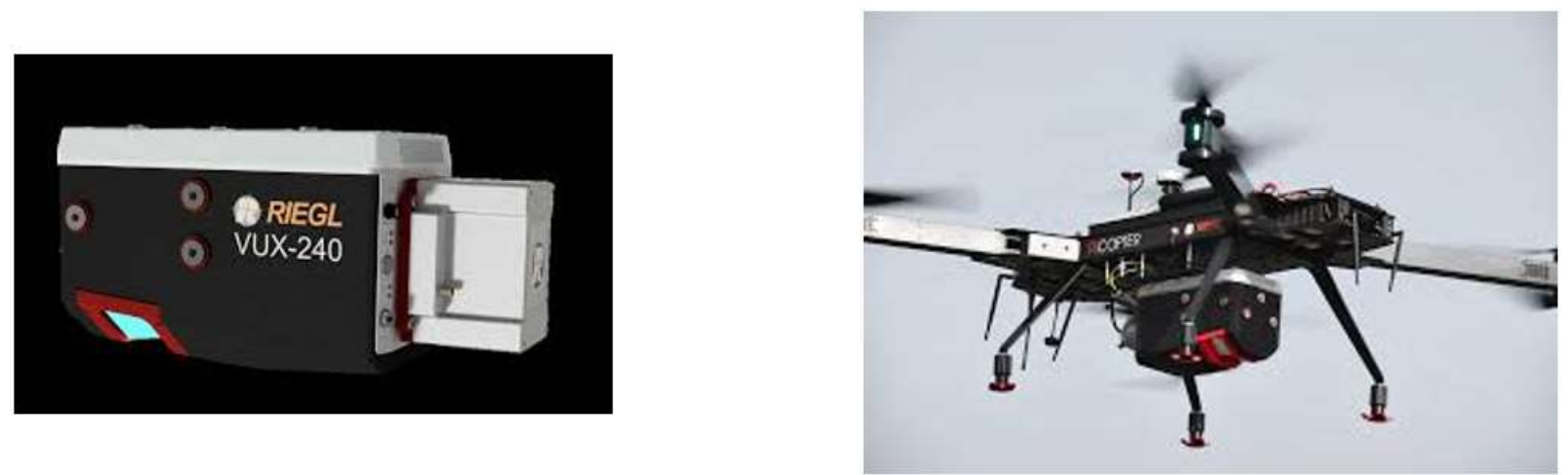

Fig 2.9: Riegl Vux-240 
Static Terrestrial LiDAR Systems (STLS) is another type of LiDAR scanners that is intended to be used in stationary mode. STLS technology is considered relatively new, as its development started in the early 2000s, mainly for surveying applications [26]. These scanners tends to have higher accuracy and richer 3D data than the MMS and ALS. Its enhanced accuracy is in the cm or even mm range [26]. The Trimble SX10, released October, 2016, has a maximum measurement range of $600 \mathrm{~m}$ and is one example of a recently developed STLS. It provides a reported accuracy of $2.5 \mathrm{~mm}$ at a $300 \mathrm{~m}$ measuring range [27]. Furthermore, the Faro focus-350 is another example of an STLS with a measuring range of $350 \mathrm{~m}$ and a stated accuracy of $1 \mathrm{~mm}$ [28].

It is worth noting that the weight of STLS is usually around $10 \mathrm{~kg}$, so the Faro focus-350 (weighing just $5 \mathrm{~kg}$ ) is considered a lightweight STLS. Moreover, Teledyne Optech recently released (March, 2017) Polaris a new STLS that can survey targets up to a 1,600 $\mathrm{m}$ range, with a stated range accuracy of $5 \mathrm{~mm}(1 \sigma$ at $100 \mathrm{~m})$. The scanner weight is $11.2 \mathrm{~kg}$ and a few of its usage applications are in civil engineering, construction, mining, geology and transportation [29].

STLS typically use indirect georeferencing techniques if real world coordinates are required. Another STLS recently (November, 2016) introduced by Leica is the BLK360, which has a very small form factor and is quite lightweight compared to the other scanners in the STLS category. The dimensions of this scanner are height $165 \mathrm{~mm}$, diameter $100 \mathrm{~mm}$, and weight $1 \mathrm{~kg}$, and it has a maximum range of $60 \mathrm{~m}$. The BLK360's stated accuracy is $7 \mathrm{~mm}(1 \sigma$ at $20 \mathrm{~m})$ [30]. All of these STLS (the Trimble SX10, the Faro focus-350, the Polaris and the BLK360) are enumerated in Fig. 2.10 .

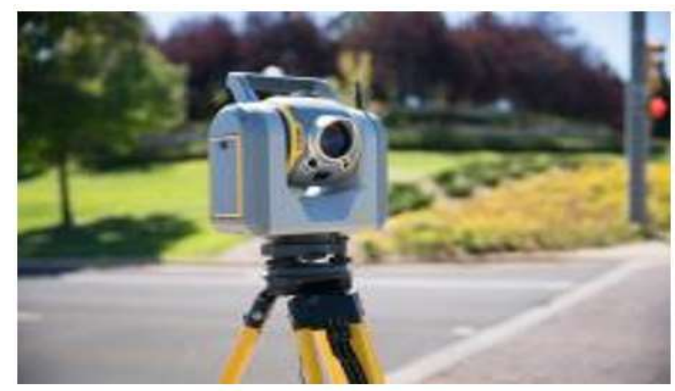

a)

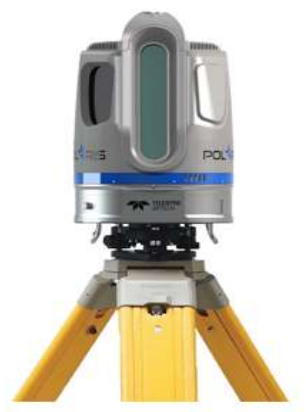

c)

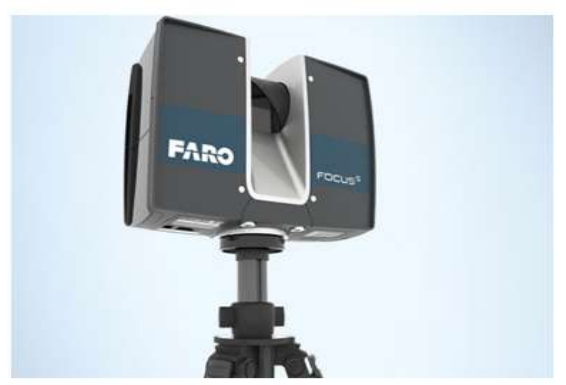

b)

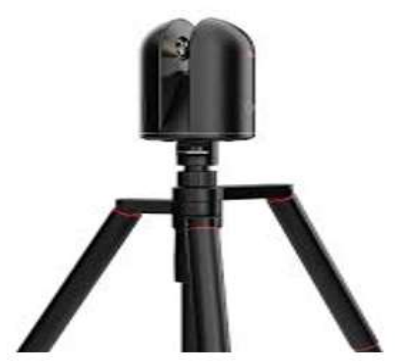

d)

Fig 2.10: Examples of different STLS, a) Trimble SX10, b) Faro Focus-350

c) Optech Polaris, and d) Leica BLK360 
As mentioned and highlighted, LiDAR-based mapping systems can be categorized according to the platform used either as MMS, ULS (a special case of ALS), and STLS. The turn-key mapping systems provided by the commercial vendors tend to be cost-prohibitive to many end-user segments and require a higher level of technicality from the operator. They also lack the flexibility that custom-made mapping systems offer regarding sensor manipulation and modification, thus leading to a limitation in the applications in which they can serve. To the best of our knowledge, a generic mapping system with crosscutting capabilities that can be readily deployed in the three different modes is non-existent. Hence, a primary objective of this dissertation is the design and development of a system architecture of a cost-efficient LiDAR-based mapping system that can be utilized in three different modes (STLS, MMS, and ULS). Such a system would not only provide the versatility of being able to be used in different modes, thereby addressing the needs of diverse end-user segments and application domains, but would also attain survey-grade accuracy.

\subsection{System orientation impact on the generated point cloud}

As discussed previously in section 2.3, there are a number of LiDAR-based mapping systems from commercial vendors, in addition to a few research attempts for developing new mapping systems that utilize the SMbL scanners. Although system orientation can help maximize the information that can be captured in the point cloud, limited information is available about the optimal system orientation for roadside surveys [31]. Moreover, after performing an exhaustive literature review in this regard, it was found that there is a lack of research efforts analyzing the impact of the mapping system orientation on the generated point cloud, more specifically on the point density, point spacing, and narrow features extraction capabilities.

Rieger et al. [32] studied the effect of MMS configurations on point density, showing a significant impact of the measurement range on the attainable density. The researchers found that the measurement range is affected by system orientation [31]. In addition, system orientation affects the characteristics of the point cloud that are needed to enable the successful features extraction through automated algorithms [33]. The work in [31-33] was performed on a 2D single beam fullcircle scanner, which exhibited a data acquisition mechanism that yields a simpler scanning pattern. On the other hand, the SMbL has a multi-beam scanning mechanism that yields a more complex scanning pattern [34], adding to the challenge of finding optimal system orientation. 


\subsection{Structure from Motion SfM Photogrammetry}

As highlighted in the previous sections, LiDAR scanners and platforms have experienced rapid technological advances in the past few years. At the same time, there has been swift progress in computer vision processes, contributing to the phases of photogrammetry workflows. Photogrammetric principles are embedded in the SfM workflow [35]. One of the landmarks in computer vision discipline is the 8-point algorithm developed by Higgins [36] for the reconstruction of a scene from two projections. Remarkably, it included only four references, one of which was the photogrammetric principle of relative orientation, as detailed in Thompson's work [37]. Hartley [38] further modified the 8-point algorithm, increasing its robustness. These classical algorithms, along with the more recent automatic feature corresponding algorithms, resemble the computer vision part in the SfM workflow. Though, the interior, exterior orientation parameters, coplanarity constraints, epipolar lines, self-calibration and bundle block adjustment entities used in the SfM workflow are deeply rooted in the photogrammetry domain.

Topographic mapping applications have traditionally dominated photogrammetry but require metric cameras and a number of well-established workflows [37]. This is traditionally associated with high capital and logistical costs [39]. One of the main photogrammetry applications is extracting 3D information from 2D imagery, but this requires overlapping imagery. SfM is used to build the 3D representation of an object by using overlapping 2D images acquired from a wide array of different viewpoints and by solving the correspondence and matching problem in photogrammetry the $3 \mathrm{D}$ representation is generated. Automatic image matching has been boosted by the influential work of Lowe [40], who developed the correspondence and matching Scale Invariant Feature Transform (SIFT) algorithm. The SIFT algorithm is invariant to change in scale, rotation, translation and to some extent is invariant to change in illumination and viewpoint [40]. It is one of the most popular and robust algorithms for solving the automatic correspondence and matching of features within images. Several other automatic image-matching techniques followed, mainly to speed up the processing time, as they are less computationally demanding. These techniques include Speed Up Robust Feature (SURF) [41], Robust Independent Elementary Features (BRIEF) [42], and Oriented Fast and Rotated (ORB) BRIEF [43].

SfM uses matching features in multiple overlapping images to solve for the interior and exterior orientation parameters and scene geometry simultaneously. This can be achieved by highly redundant matched features, along with a bundle block adjustment process [39]. Furthermore, camera-posing information and/or a sufficient number of ground control points are not a prerequisite to solve for scene triangulation and reconstruction in the SfM workflow. Nevertheless, in the absence of this information, the output of SfM will lack the scale and orientation provided by ground-control coordinates or the external camera pose measurements by a GNSS sensor [39]. The result of applying SfM only is a sparse 3D point cloud, which is typically followed by a multiview stereopsis (MVS) algorithm to densify the 3D point cloud to several orders of magnitude [35]. Knowing the camera parameters from the SfM workflow helps to simplify the matching between pixels to that constraint with the epipolar geometry. Thus, instead of doing a 2D search between pairs of images to find pixel correspondence, the search is reduced to a 1D search along the epipolar line. One of the most used MVS algorithms is the Patch MultiView Stereopsis (PMVS) [44]. 
The SfM algorithm consists of three main steps. The first step is to find the correspondence between features in the collected overlapping imagery. The Scale Invariant Feature Transform (SIFT) algorithm [40] is used to find the correspondence of features in the collected imagery. The second step is to calculate relative orientation and camera poses in an arbitrary 3D coordinate system after defining the tracks of matched features between images. The third step is to further refine the second step's output through Bundle Block Adjustment (BBA). SfM algorithm workflow, as defined in [45], can be summarized as follows.

The SIFT is used to solve the correspondence and matching of features within images. The algorithm starts by finding key-points. These are distinct points that can be localized within the image. This is achieved by applying Gaussian smoothing with different standard deviation, as shown in Equation 2.4. The smoothing is performed by convolving the original image with the Gaussian kernels and then applying the difference of Gaussians DoG between the smoothed images, according to Equation 2.5. This will find extrema (maximum or minimum) in the image space.

$$
G(x, y)=\frac{1}{2 \pi \sigma^{2}} e^{-\frac{x^{2}+y^{2}}{2 \sigma^{2}}}
$$

Where

$\mathrm{x}$ is the horizontal distance from the origin,

$\mathrm{y}$ is the vertical distance from the origin,

$\sigma$ is the standard deviation

The difference of Gaussian is calculated from

Where

$$
G_{\sigma, k \sigma}(x, y)=I * \frac{1}{2 \pi \sigma^{2}} e^{-\frac{x^{2}+y^{2}}{2 \sigma^{2}}}-I * \frac{1}{2 \pi k^{2} \sigma^{2}} e^{-\frac{x^{2}+y^{2}}{2 k^{2} \sigma^{2}}}
$$

$\mathrm{x}$ is the horizontal distance from the origin,

$\mathrm{y}$ is the vertical distance from the origin,

$\sigma$ is the standard deviation

I is the image intensity

$\mathrm{k}$ is a constant factor $>1$

Afterwards, pyramid layers are calculated to resemble different scales. The Gaussian smoothing is applied to the scaled images, followed by the difference of Gaussians of the scaled smoothed images. The extrema (maximum or minimum) are now calculated in the scale space. Thus, the distinct local key-points in image and scale space can now be identified. Figure 2.11 summarizes the steps for key-point identification. 


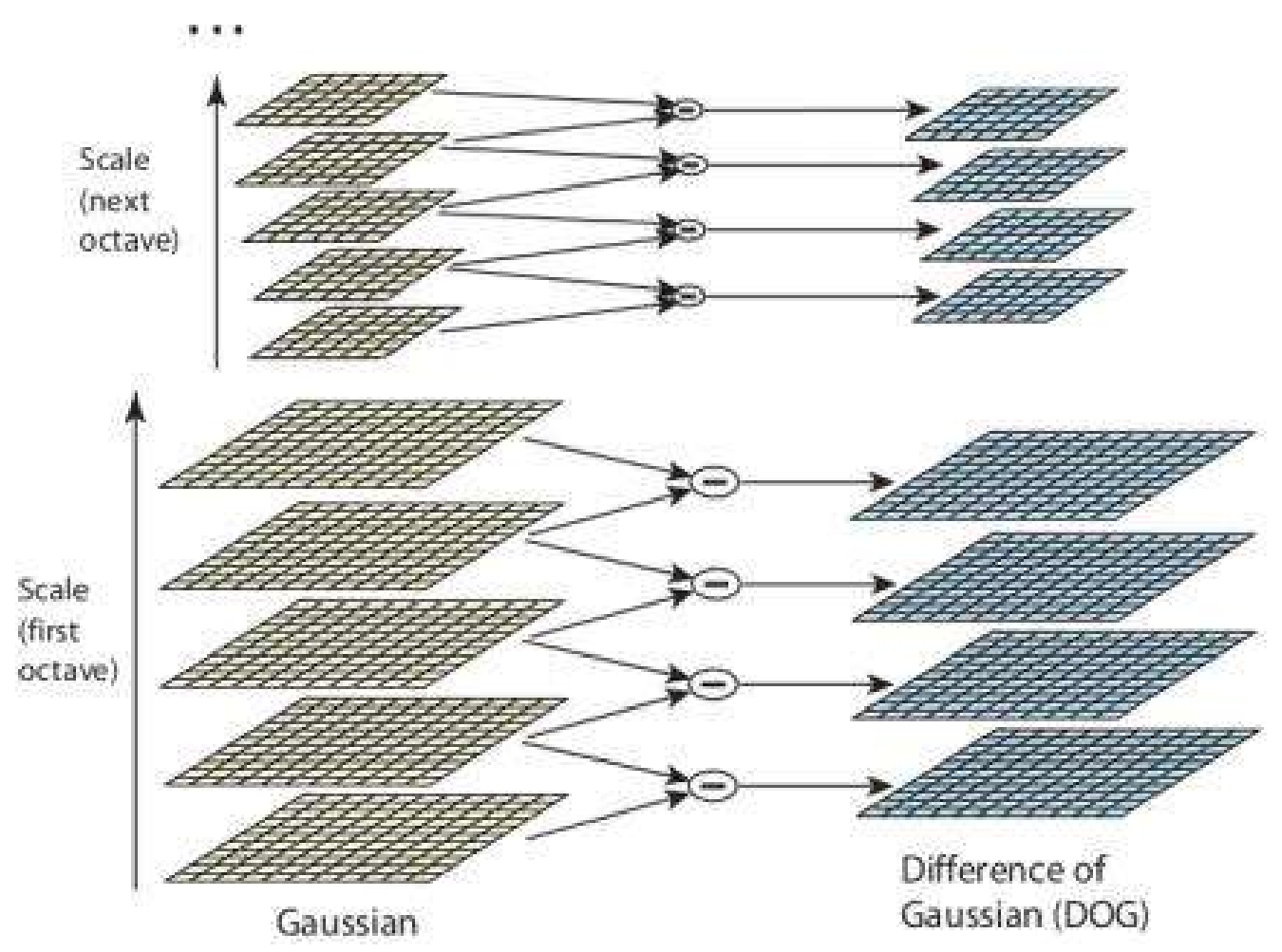

Fig 2.11: SIFT key-points identification (adopted from [40])

\section{Key-point descriptor}

The local 16*16 pixels around the key-point is used to build the key-point descriptor. The gradient direction is calculated for each pixel in the $16^{*} 16$ window. Then, a $4 * 4$ histogram with an 8 bin orientation (every $45^{\circ}$ and normalized) is calculated, forming an $8 * 4 * 4=128$ vector descriptor. Figure 2.12 shows the key-point descriptor according to [40]. 


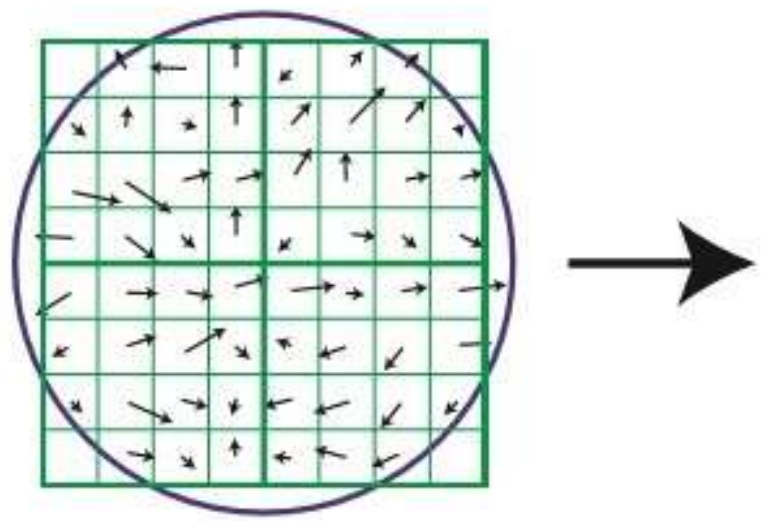

Image gradients

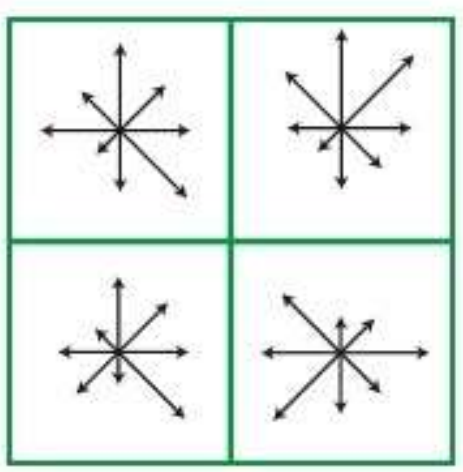

Keypoint descriptor

A keypoint descriptor is created by first computing the gradient magnitude and orientation at each image sample point in a region around the keypoint location, as shown on the left. These are weighted by a Gaussian window, indicated by the overlaid circle. These samples are then accumulated into orientation histograms summarizing the contents over $4 \mathrm{x} 4$ subregions, as shown on the right, with the length of each arrow corresponding to the sum of the gradient magnitudes near that direction within the region. This figure shows a $2 \times 2$ descriptor array computed from an $8 \times 8$ set of samples, whereas the experiments in this paper use $4 \times 4$ descriptors computed from a $16 \times 16$ sample array.

Fig 2.12: Key point descriptor (adopted from [40])

The next step, after identifying the key- points and defining the key-point descriptor, is the matching of key-points between images. The matching is performed by comparing the key-point descriptors and finding the nearest neighbor in the descriptor space. Let a key-point $\mathrm{z}$ in image I denoted by I (z) be compared to the key-points in image J. Only correct correspondences will satisfy the epipolar constraint. The epipolar constraint relates the corresponding features between the two pairs of images through the fundamental matrix F. The F-matrix, shown in Equation 2.6, encompasses the relative orientation and positions of the two cameras and the internal camera settings.

$$
\left[\begin{array}{lll}
x^{\prime} & y^{\prime} & 1
\end{array}\right] F\left[\begin{array}{l}
x \\
y \\
1
\end{array}\right]=0
$$

where x'y' and $x$ y are the feature coordinates in the images. RANdom SAmple Consensus (RANSAC) is used to model-fit the F-matrix and identify the correct matching correspondences. For every camera position, a set of six exterior orientation parameters and three interior orientation parameters are defined. For every correct matched correspondence, a set of three coordinates X, $\mathrm{Y}, \mathrm{Z}$ is defined. Tracks of corresponding features are calculated by creating a chain-like correspondence between the same features appearing in different images. The Bundle Block Adjustment (BBA) with self-calibration is then used as a non-linear least-square optimization to solve for the scene geometry and cameras pose information simultaneously by minimizing the reprojection errors of the features tracks presented in Equation 2.7, thereby solving the SfM problem. The BBA reprojection error is also shown in Fig. 2.13 


$$
G(C, X)=\sum_{i=1}^{n} \quad \sum_{j=1}^{m} w i j\left\|q_{i j}-P\left(C_{i}, X_{j}\right)\right\|^{2}
$$

Where

$\mathrm{n}$ is the number of views,

$\mathrm{m}$ is the number of tracks

wij=1 if $\mathrm{Ci}$ see $\mathrm{Xj}$ or zero otherwise

$\left\|\mathrm{q}_{\mathrm{ij}} \mathrm{P}\left(\mathrm{C}_{\mathrm{i}}, \mathrm{Xj}\right)\right\|^{2}$ is the reprojection error

$\mathrm{P}\left(\mathrm{C}_{\mathrm{i}}, \mathrm{Xj}\right)$ is the projection of $\mathrm{Xj}$ on the image of camera $\mathrm{Ci}$ through the perspective projection equations, where $\mathrm{Xj}$ is converted to the camera's coordinate system through a rigid transformation Equation 2.8, followed by perspective division and scaling with focal length $\mathrm{f}$, as shown in Equation 2.9.

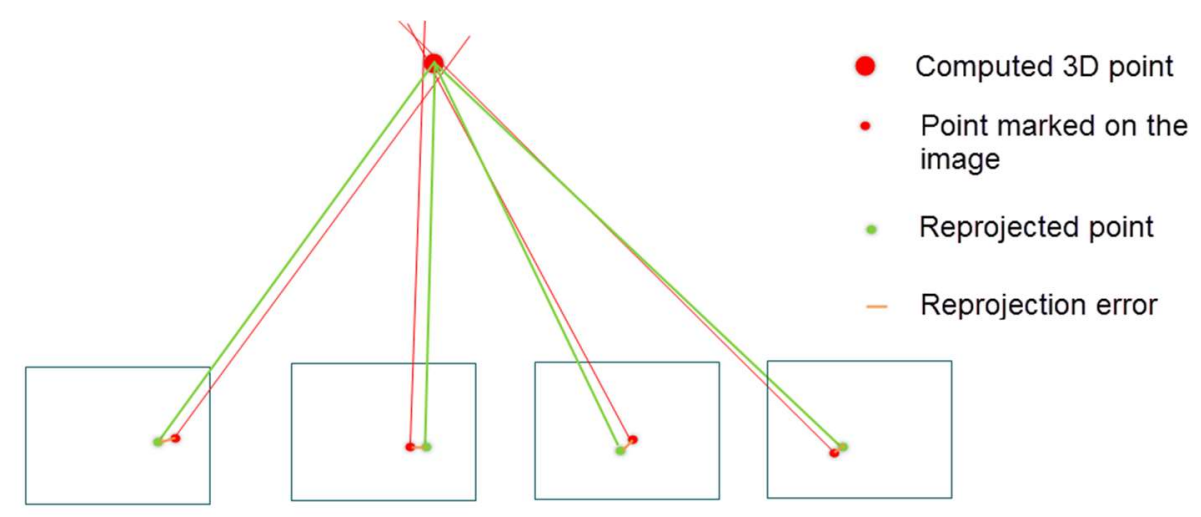

Fig 2.13: BBA Reprojection error

$$
\begin{aligned}
X^{\prime} & =\left[\begin{array}{l}
X^{\prime}{ }_{x} \\
X^{\prime}{ }_{y} \\
X^{\prime}{ }_{z}
\end{array}\right]=\mathrm{Ri}(\mathrm{Xj}-\mathrm{ci}) \\
x^{\prime} & =\left[\begin{array}{c}
\frac{f X^{\prime} x}{X^{\prime} z_{z}} \\
\frac{f X^{\prime}{ }_{y}}{X_{z}}
\end{array}\right]
\end{aligned}
$$

$\mathrm{x}^{\prime}$ is a $2 \mathrm{D}$ point on the image with coordinates $\left(\mathrm{x}^{\prime}{ }_{\mathrm{x}}, \mathrm{x}_{\mathrm{y}}{ }_{\mathrm{y}}\right)$. Sparse BBA is then implemented to solve for the scene geometry and camera poses while minimizing the reprojection error. The aforementioned steps are presented in detail in [45]. 


\section{Multi-view stereopsis MVS}

The SfM algorithm output is a sparse point cloud. This sparse point cloud is used as an input for MVS that increases the point cloud by about two orders of magnitude [35]. Knowing the camera parameters from the SfM workflow simplifies the matching between pixels to that constraint with the epipolar geometry. Thus, instead of doing a 2D search between pair of images to find pixel correspondence, the search is reduced to a 1D search along the epipolar line. The point correspondence may be densified up to each pixel in the image. One of the most used MVS algorithms is the Patch Multiview Stereopsis (PMVS).

The PMVS algorithm, as presented in [44], can be summarized as follows. The algorithm is defined through its main three steps: initial feature matching, patch expansion, and patch filtering. The expansion and filtering are iterated thrice to densify the patches and remove erroneous ones.

The first step is to apply the DoG and Harris operator to find corner features. For every feature $\mathrm{f}$ in the image Ii, the corresponding DoG/Harris feature along the epipolar line within two pixels are selected $\mathrm{f}^{\prime}$. Triangulation is done between ( $\left.\mathrm{f}, \mathrm{f}^{\prime}\right)$ to their $3 \mathrm{D}$ points. A patch candidate center $\mathrm{c}(\mathrm{p})$ is initialized from the (f, $\left.\mathrm{f}^{\prime}\right)$ triangulation and the normal $\mathrm{n}(\mathrm{p})$ is initialized from Equation 2.10.

$$
n(p) \text { from } \overrightarrow{c(p) O(I l) /} \overrightarrow{|c(p) O(I l)|}
$$

where $\mathrm{O}$ (Ii) is the optical center of the image Ii. The $\mathrm{c}(\mathrm{p})$ and $\mathrm{n}(\mathrm{p})$ parameters are then optimized by considering the visible images of $p, v(p)$. The images retained from the visible imagery are the ones with photo-consistency measurements with an Ii $>$ threshold. The patch is kept if the retained number of images is $>3$

The second step is the expansion step, which aims to find a patch for every image cell $\mathrm{Ci}(\mathrm{x}, \mathrm{y})$, where $\operatorname{Ci}(\mathrm{x}, \mathrm{y})$ is $2 * 2$ grid of pixels. Existing patches are used to generate new patches in empty places. The neighboring empty cells (p) to an existing patch $\mathrm{p}$ is identified, as in Equation 2.11.

$$
\operatorname{Cells}(p)=\left\{C i\left(x^{\prime}, y^{\prime}\right)\left|p \in Q i(x, y), Q i\left(x^{\prime}, y^{\prime}\right)=\emptyset,\right| x-x^{\prime}|+| y-y^{\prime} \mid=1\right\}
$$

where Qi(x, y) forms the set of patches that project to the cell $\mathrm{Ci}(\mathrm{x}, \mathrm{y})$. To generate a new patch $\mathrm{p}^{\prime}$, its $\mathrm{n}\left(\mathrm{p}^{\prime}\right)$ and $\mathrm{v}\left(\mathrm{p}^{\prime}\right)$ are initialized by the values for $\mathrm{p}$, while $\mathrm{c}\left(\mathrm{p}^{\prime}\right)$ is initialized as the point of intersection between the ray passing through the center of $\mathrm{Ci}(\mathrm{x}, \mathrm{y})$ and the plane containing $\mathrm{p}$. Then $c\left(p^{\prime}\right)$ and $n\left(p^{\prime}\right)$ are refined as shown previously. Images are removed from v (p') if their average pairwise photo-consistency is less than a threshold, and images are added if pairwise photoconsistency is more than the threshold. If the remaining images in $v\left(p^{\prime}\right)$ are $>3$, the patch is created and the patch set updated.

The third step is the filtering step. Two filters are used to filter out any erroneous patches that may arise from the previous two steps. The first filter uses visibility consistency to filter out erroneous patches. For patches p and p' to be neighbors, the distance along the normal should be less than a threshold, as shown in Equation 2.12.

$$
\left|\left(c(p)-c\left(p^{\prime}\right)\right) \cdot n(p)\right|+\left|\left(c(p)-c\left(p^{\prime}\right)\right) \cdot n\left(p^{\prime}\right)\right|<\gamma d
$$


Let $U(p)$ be the set of patches p' that do not satisfy Equation 2.12. Here, p. p' is shown in the same cell in one of the images where $p$ is visible. However, $p$ is filtered out if the inequality (Equation 2.13) is satisfied.

$$
|\mathrm{v}(\mathrm{p})|(1-\mathrm{C}(\mathrm{p}))<\sum_{p_{i \in U(p)}} 1-C\left(p_{i}\right)
$$

where $\mathrm{C}(\mathrm{p})$ is the average pairwise photo-consistency of $\mathrm{p}$. The second filter involves applying a weak form of regularization. If the proportion of neighboring patches to $p$ is $<0.25$ of the number of patches in the same cell as $p$ (plus its adjacent cells in all images in which $p$ is visible), the patch is filtered out. The output of the MVS is a dense point cloud.

As it has been highlighted, a key step in the SfM workflow is the correspondence and matching of features between different images. A successful SfM-MVS depends on the correct correspondences between features in different images; thus, its performance is failure prone in homogeneous areas (areas with poor textures) [39, 47-50]. In addition, the presence of vegetation cover adversely affects the accuracy of the generated point cloud from the SfM workflow [51, 52]. Moreover, Furukawa et al [44] lists the MVS algorithm limitations as its need for reliable texture information and that the algorithm does not perform well in narrow baseline cases. Thus, in the present research, a new data acquisition and fusion framework between the developed MMS and the SfM-MVS workflow is proposed to enhance the $3 \mathrm{D}$ reconstruction in such challenging scenarios. The following section presents a literature review of the state-of-the-art LiDAR and optical imagery fusion approaches, in addition to DSM/DEM generation.

\subsection{LiDAR and Optical imagery data Fusion}

Due to the complementary nature of the LiDAR data and optical imagery, a number of studies have exploited the fusion between these data sources to infer more accurate information [53]. Gneeniss et al. [54] utilized a robust least square surface matching algorithm to align the photogrammetric data to the LiDAR-based induced surface and extract LiDAR control points accordingly, which are then used in bundle adjustment to calibrate the optical camera. Lin et al. [55] introduced an efficient 3D registration method based on matching key features between the optical 2D images and 2D bearing angle images transferred from the point cloud. Persad and Armenakis [56] used a wavelet kernel, a log-polar descriptor for the key points and an NNDR (Nearest Neighbor Distance Ratio) to register optical-based DSM and LiDAR-based DSM. Li et al. [18] utilized SfM to enhance the trajectory used for the LiDAR point cloud georeferencing. Zieher et al. [53] employed a voxel-based approach with an expert-based rules criteria to integrate the point clouds from optical and LiDAR data for landslide monitoring.

Rizeei and Pradhan [57] proposed a 3D rational polynomial coefficients 3D RPC model for ortho rectifying a very high resolution imagery of the Worldview-3 satellite using derived ground control points GCP from LiDAR-based generated DSM. Yao et al. [58] registered LiDAR data and panoramic camera images through the selection of key points within different blocks of the point cloud. Javanmardi et al. [59] applied road-based features to register LiDAR-based data to optical data through a Gaussian mixture model GMM and a normal distribution transform NDT. Zhang et al. [60] used terrestrial façade images to generate a 3D point cloud and incorporated a modified coherent point drift algorithm with (surface) normal consistency to use 2D points outlined on 
buildings to register the optical data to ALS data. Berrio et al. [61] utilized a deep neural network to extract semantic information from optical imagery and superimpose that information on the LiDAR point cloud.

Habib et al. [62] extracted linear features from optical imagery and LiDAR datasets and used the extracted linear features in the registration between the LiDAR and optical data. Besides, Tochi et al. [63] investigated the precision of a hybrid sensor (the Leica citymapper), which consists of an active LiDAR and passive optical cameras, an all-in-one mapping solution, in two study areas reporting a $3 \mathrm{~cm}$ deviation between the LiDAR data point cloud and the optical data dense image matching DIM point cloud.

As can be deducted from the aforementioned studies, the extraction of conjugate features is mainly a prerequisite for the fusion process which may be suited to urban areas, where distinct features such as corners, building outlines and linear features are abundant. These features can be scarce in rural or other environments, hindering the applicability of these methods in such challenging environments. An alternative approach to the data fusion process would be the utilization of a very expensive hybrid sensor, as was done in [63], which may be out of reach for many end-users. Hence, in this research, a data fusion framework is proposed that utilizes the developed costefficient LiDAR-based mapping system and the SfM-MVS workflow, thus increasing the generality of the framework application in different environments and its utilization by diverse end-users.

\subsection{DSM and DEM generation Approaches}

High-resolution DSM and DTM are crucial geospatial products [64] that are used in many applications, such as hydrology [50,65-66], 3D city modeling and building change detection [67], geomorphology [68-69], glaciology [46, 70-71], modelling of mass movements, telecommunication, and many others [72]. A variety of methods may be applied to obtain elevation data used in DSM generation, with the approaches differing with regard to cost, equipment, labor and time requirements. The resolution and accuracy of the generated DSM depend on the method used, the complexity of the area, and the sampling interval.

The two main remote sensing techniques typically used for DSM generation are stereo imagery photogrammetry and LiDAR. In the 1980s and 1990s, optical imagery photogrammetry was the standard practice used for DSM generation, whereas over the past two decades, advances in LiDAR technology have made it the standard practice for DSM generation. Although very high resolution satellite stereo imagery is still used for DSM generation [73-74], the so-called standard practice for DSM generation is the utilization of LiDAR data [48, 75-79]. Millions or even billions of non-selective sampling points can be produced by using LiDAR scanners, whether airborne LiDAR ALS, Mobile LiDAR MMS or terrestrial LiDAR STLS [34, 48]. However, one main drawback of using such scanners is the associated high capital and operational costs [34, 50], especially if temporal analysis is needed. The measured sampling points are then converted into a regular grid representing DSM, which is a $2.5 \mathrm{D}$ representation of the environment, with each pixel storing the elevation $[62,80]$. 
In recent years, stereo imagery photogrammetry has gained momentum due to advances in the computer vision field. New approaches include automatic image matching techniques like SIFT and SURF, in addition to MVS algorithms and SfM photogrammetric techniques. One reason why SfM-MVS techniques have gained such momentum is that their output is $3 \mathrm{D}$ point cloud with higher point density than that from LiDAR scanners, but at a fraction of the cost and effort [48, $50,81]$. Another key advantage of the SfM is the greater number of viewpoints used, which minimize the occlusion and shadowing that may be experienced with STLS [34].

Nevertheless, reliable results are dependent on the accurate correspondence and matching of features between images $[62,82]$. Structure from motion as a tool for topographic mapping was used in a number of studies. Leitão et al. [83] generated a fine $(5 \mathrm{~cm})$ DSM that was used for overland flow modelling in urban areas, reporting 0.1 to $0.2 \mathrm{~m}$ vertical accuracy utilizing stereo imagery captured from a UAV for the DSM generation. Ouedraogo et al. [84] mapped the micro topography of an agriculture watershed using a generated $1 \mathrm{~m}$ resolution DEM and reporting a 0.5 m vertical RMSE.

Furthermore, Tejada et al. [85] was able to quantify tree heights from the DSM with a $0.35 \mathrm{~m}$ RMSE, while Reu et al. [86] conducted a thorough analysis of an archaeological site, generating a $3 \mathrm{~cm}$ resolution DSM and reporting a vertical accuracy of $0.06 \mathrm{~m}$. In addition, Ajayi et al. [87] was able to generate a DSM with a vertical accuracy of $0.1 \mathrm{~m}$ using images with approximately 15 $20 \%$ side overlap, while Aguera-Vera et al. [88] combined UAV images acquired horizontally and at a $45^{\circ}$ tilt to study a near-vertical road cut-slope.

One should note, however, that the environments where the above-referenced studies were applied are generally considered rich-textured. Another concern regarding these studies is the black-box approach used in applying the UAV-SfM algorithm. In such an approach, very few details are known about the underlying algorithms used, thus hindering a full understanding of the processing steps as well as the types of errors and their impact on the final product [89].

In order to cover every aspect, the Digital Elevation Model (DEM) generation method is also described. DEM, which is a bare land elevation model, can be extracted from the raw data point cloud or from the generated DSM. DEM extraction algorithms can be designated as slope-based or linear-prediction-based, according to the morphological filtering.

Moreover, directional scanning filters have also proved to be capable algorithms for DEM generation from ALS data [80]. A slope-based algorithm depends on the assumption that the slope change between ground points is less than the slope change between ground and non-ground points, while linear-prediction-based algorithms start by assuming rough terrain and height differences between points. The estimated rough terrain is then minimized by linear least squares interpolation. Classical morphological filtering such as erosion and dilation have been commonly implemented in DEM extraction [80].

Alternatively, the extraction of DEM from the raw 3D point cloud can be achieved by using filters such as i) Multiscale Curvature Classification (MCC), ii) surface-based filtering, iii) progressive TIN-based, or iv) physical simulation processing (Cloth Simulation Filtering [CSF]) [90]. The MCC filtering algorithm procedure begins by determining the vertical component $(Z)$, after which 
the thin plate spline interpolation is used to generate a surface model with user-defined scale parameters and tolerance. The surface-based filtering method begins by giving weight to individual points. It commences by assuming the whole point cloud is ground, after which conditions are checked iteratively and residuals are calculated for the points. The points satisfying the conditions are kept as ground; otherwise, they are labelled as non-ground. One disadvantage of the surfacebased filtering method is that it needs the user to define some parameters for the conditions generation, which requires that the user have some sort of experience with the field data.

In the progressive TIN-based approach, tiles or point cloud data blocks are created, while for each data block, points with the lowest elevation are selected as seed points. These seed points are then used to generate TIN surfaces. A distance and a height threshold for the points to these TIN surfaces are used to segment the points to either ground or non-ground. Lastly, the CSF strategy starts by inverting the LiDAR point cloud and simulating a cloth surface on the inverted point cloud, and then intersecting the generated surface with the LiDAR nodes. This yields the ground points, which are the points that are limited to move down [90].

Although there are a number of studies that used the multimodal point clouds from LiDAR-based and Dense Image Matching (DIM) workflows, the majority of the studies used the LiDAR-based data as a reference to the DIM data, whereas only a few studies used the multimodal data for comparison purposes [63]. As a consequence, and to test the ability of the developed system to produce derived data such as DSM, the generation of DSM surfaces from the two multimodal data is investigated and compared. 


\section{LIDAR-BASED PLATFORM-INDEPENDENT MAPPING SYSTEM ARCHITECTURE DEVELOPMENT}

\subsection{Introduction}

As discussed in the previous chapters, there is an ever-increasing demand for 3D mapping from diverse end-user segments towards a variety of applications. Due to technical and financial constraints, the existing traditional mapping systems are out of reach for many end-users. Moreover, the current mapping systems are designed to be utilized in one mode of operation, whether ULS, MMS or STLS. A generic mapping system that is accurate, cost-efficient, and can readily be used in the three aforementioned mapping modes is currently non-existent. Such a system would meet many end-user requirements and could be deployed across numerous application domains. The development of such a system is far from being easy, however, as several different challenges need to be addressed, especially with regard to the usage of low-end sensors, in order to be cost-efficient while maintaining surveying-grade accuracy. The challenge becomes even more complex, given the need for such a system to be platform-independent and to follow a plug-n-go concept. Furthermore, it has to be highly versatile in order to be easily deployed in the different acquisition modes in addition to having optimum SWaP characteristics to meet stringent ULS requirements. As well, the system should be modular to allow for extra sensors to be easily added, and should allow minimal modification to extend its applicability to indoor mapping. This chapter presents the development of the system architecture for a generic LiDAR-based mapping system, starting with the optimization of sensor selection, placement, synchronization, and integration at the Hardware (HW) and Software (SW) levels.

\subsection{Optimized Selection of Sensors}

The main building blocks of a mapping system that utilizes the Direct Georeferencing (DG) approach in 3D mapping are: i) the Remote Sensing Sensor (RSS), ii) the GNSS/INS module, and iii) the system management unit. The RSS may be an active and/or a passive sensor. Passive sensors may include digital cameras (monocular or stereo), multispectral cameras, or hyperspectral cameras, while active sensors may include LiDAR or radar sensors. The GNSS/INS module consists of a GNSS receiver and an Inertial Navigation System (INS). Higher accuracy is attained with multi-frequency GNSS receivers, as more errors can be corrected, such as ionospheric delay, which is corrected through ionosphere-free linear combinations. Moreover, with multi-frequency receivers, the GNSS signal is more immune to interference. In addition, if the GNSS receiver is a multi-constellation receiver, it can access signals from several constellations, such as GPS, GLONASS, BeiDou and Galileo, resulting in an increased number of satellites within the receiver field of view. The increase in the number of satellites that can be tracked has several benefits, such as reduced signal acquisition time, improved position and time accuracy and improved distribution of satellite geometry, resulting in improved dilution of precision.

An INS consists of an Inertial Measurement Unit (IMU) and a computer chip that uses the IMU readings to provide the position, orientation, and velocity relative to a known starting point. An IMU uses a number of accelerometers and gyros in a defined orientation in order to measure the movement of its body in 3D space [91]. Although there is no standard categorization of IMU types, they can generally be grouped according to their performance into four group types: marine and navigation grade, tactical grade, industrial grade, and consumer grade. Marine grade inertial 
sensors are the most expensive, but they also provide the highest performance. As expected, the lower the performance provided by the other IMU group types, the less their cost.

There are three main technologies used for IMU manufacturing, namely, the Ring Laser Gyros (RLG), the Fiber Optic Gyros (FOG), and the Micro Electro-Mechanical System (MEMS)-based gyros. The RLG are generally the most accurate, but are very expensive. The FOG sensors provide a lower-cost alternative to the RLG and give a high level of accuracy, but they are still relatively expensive and have a large form-factor and high power demands. The MEMS, on the other hand, are not only less expensive but generally have a small form-factor and consume less power. Moreover, with recent technology advances, MEMS have closed the performance gap on some FOGs. It is worth noting that the GNSS/INS module is generally the most expensive entity in a mapping system and is crucial for accurate $3 \mathrm{D}$ reconstruction of a mapped environment. The system management unit is responsible for the mapping system control and provides storage for the data collected by the sensory RSS and the GNSS/INS module.

\subsubsection{LiDAR RSS optimized selection}

LiDAR scanners are active RSS sensors. The LiDAR sensor measures the range to the surrounding environment. Hence, with precisely known azimuth and elevation angle of the LiDAR beam, the $3 \mathrm{D}$ coordinates of the illuminated target point can be calculated. The requirements for the autonomous vehicle domain stimulated the development of new LiDAR scanners. The new LiDAR scanners are generally lightweight, compact and relatively low-cost, making them an attractive option to be considered for the mapping system RSS.

The latest LiDAR scanners can be classified as either solid-state or SMbL. The solid-state versions are lower cost and have a smaller form-factor, but because of their unsuitability for 3D mapping (they are built to provide grid occupancy only), they were not considered in this research. As the technology advances, the solid-state LiDAR is a promising technology that may cause an archetype shift for the 3D mapping domain in the near future. Regarding the SMbL and the task at hand of selecting a LiDAR RSS for the mapping system, a number of commensurate SMbL sensors were investigated. More specifically, the Velodyne HDL-32E, the VLP-16, and the Quanergy M8 were compared. Tables 3.1[5], 3.2[5] and 3.3[6] show the specifications of the HDL-32E, the VLP16 and the M8 sensors, respectively, while Fig. 3.1 depicts the three scanners. 
Table 3.1: The HDL-32E specifications overview

\begin{tabular}{|l|l|}
\hline \multicolumn{1}{|c|}{ Attribute } & \multicolumn{1}{c|}{ Value } \\
\hline Number of LiDAR beams & 32 \\
\hline Range & $100 \mathrm{~m}$ \\
\hline Accuracy & $\pm 2 \mathrm{~cm}$ \\
\hline Vertical field of view (VFOV) & $+10^{\circ}$ to $-30^{\circ}$ \\
\hline Horizontal field of view (HFOV) & $360^{\circ}$ \\
\hline Spinning frequency & $5-20 \mathrm{~Hz}$ \\
\hline Angular resolution (horizontal) & $0.1^{\circ}-0.4^{\circ}$ \\
\hline Angular resolution (vertical) & $1.33^{\circ}$ \\
\hline Data Points Per Second & 695,000 points/sec (single return) \\
& $1,390,000$ points/sec (dual return) \\
\hline Power consumption & $12 \mathrm{w}$ \\
\hline Weight & $1 \mathrm{Kg}$ \\
\hline Scanner Diameter/Height & $85.3 \mathrm{~mm} / 144.2 \mathrm{~mm}$ \\
\hline LiDAR class type & Class 1 Eye-safe per IEC $60825-1: 2007 \&$ \\
& 2014 \\
\hline Wavelength used & $903 \mathrm{~nm}$ \\
\hline Operating Temperature & $-10^{\circ} \mathrm{C}$ to $+60^{\circ} \mathrm{C}$ \\
\hline
\end{tabular}

An overview of the specification of the VLP-16 scanner is provided in Table 3.2 below

Table 3.2: The VLP-16 specifications overview

\begin{tabular}{|l|l|}
\hline \multicolumn{1}{|c|}{ Attribute } & \multicolumn{1}{c|}{ Value } \\
\hline Number of LiDAR beams & 16 \\
\hline Range & $100 \mathrm{~m}$ \\
\hline Accuracy & $\pm 3 \mathrm{~cm}$ \\
\hline Vertical field of view (VFOV) & $+15^{\circ}$ to $-15^{\circ}$ \\
\hline Horizontal field of view (HFOV) & $360^{\circ}$ \\
\hline Spinning frequency & $5-20 \mathrm{~Hz}$ \\
\hline Angular resolution (horizontal) & $0.1^{\circ}-0.4^{\circ}$ \\
\hline Angular resolution (vertical) & $2^{\circ}$ \\
\hline Data Points Per Second & 300,000 points/sec (single return) \\
& 600,000 points/sec (dual return) \\
\hline Power consumption & $8 \mathrm{w}$ \\
\hline
\end{tabular}




\begin{tabular}{|l|l|}
\hline Weight & $830 \mathrm{~g}$ \\
\hline Scanner Diameter/Height & $103.3 \mathrm{~mm} / 71.7 \mathrm{~mm}$ \\
\hline LiDAR class type & Class 1 Eye-safe per IEC 60825-1:2007 \& \\
& 2014 \\
\hline Wavelength used & $903 \mathrm{~nm}$ \\
\hline Operating Temperature & $-10^{\circ} \mathrm{C}$ to $+60^{\circ} \mathrm{C}$ \\
\hline
\end{tabular}

An overview of M8 specifications is provided in Table 3.3 below.

Table 3.3: The M8 specifications overview

\begin{tabular}{|l|l|}
\hline \multicolumn{1}{|c|}{ Attribute } & \multicolumn{1}{c|}{ Value } \\
\hline Number of LiDAR beams & 8 \\
\hline Range & $100 \mathrm{~m}$ \\
\hline Accuracy & $\pm 5 \mathrm{~cm}$ \\
\hline Vertical field of view (VFOV) & $+3^{\circ}$ to $-17^{\circ}$ \\
\hline Horizontal field of view (HFOV) & $360^{\circ}$ \\
\hline Spinning frequency & $5-30 \mathrm{~Hz}$ \\
\hline Angular resolution (horizontal) & $0.03^{\circ}-0.13^{\circ}$ \\
\hline Angular resolution (vertical) & $3^{\circ}$ \\
\hline Data Points Per Second & 420,000 points/sec (single return) \\
& 840,000 points $/ \mathrm{sec}$ (dual return) \\
\hline Power consumption & $1,260,000$ points/sec (triple return) \\
\hline Weight & $18 \mathrm{w}$ \\
\hline Scanner Diameter/Height & $900 \mathrm{~g}$ \\
\hline LiDAR class type & $103 \mathrm{~mm} / 87 \mathrm{~mm}$ \\
\hline Wavelength used & $\begin{array}{l}\text { Class } 1 \text { Eye-safe per IEC } 60825-1: 2007 ~ \& \\
2014\end{array}$ \\
\hline Operating Temperature & $905 \mathrm{~nm}$ \\
\hline
\end{tabular}


By investigating the specifications of the three scanners, as shown in Tables 3.1, 3.2 and 3.3, the HDL-32E provides the highest accuracy and number of LiDAR beams with the best vertical angular resolution among the three scanners. However, this scanner also has the largest formfactor, heaviest weight, and a power consumption that is $50 \%$ higher than that of the VLP-16. Furthermore, it is the most expensive sensor among the three scanners. On the other hand, the M8, though providing more points/sec compared to the VLP-16, along with triple returns, has only 8 beams and thus can only scan among 8 planes. It also has the worst vertical angular resolution $\left(3^{\circ}\right)$ and the highest power consumption, more than double the power consumption of the VLP-16. In addition, the M8 provides a field of view of only $3^{\circ}$ above the sensor horizon compared to the VLP-16's field of view of $15^{\circ}$ above the horizon.

Based on the above comparison, the VLP-16 was found to be a cost-effective alternative for the mapping system RSS, given that it features the best Size, Weight And Power (SWaP) characteristics, which is crucial for the ULS. Moreover, it provides the largest field of view above the sensor horizon $\left(+15^{\circ}\right)$ among the three compared scanners and gives a very adequate accuracy of $\pm 3 \mathrm{~cm}$. Nonetheless, due to continuous technological advances, a SMbL scanner has been introduced recently (the Velodyne VLS-128) which features 128 LiDAR beams and a measuring range of $300 \mathrm{~m}$. However, as with many new devices, the sensor is very expensive (around 150,000 USD, which is almost twenty times more expensive than the VLP-16). Another recently introduced sensor is the VLP-16 LITE, which features the exact same specifications as the VLP-16 but with a lighter weight (only $530 \mathrm{~g}$ ). It is worth highlighting that one aspect of the design criteria for the system architecture proposed in this dissertation is its flexibility to easily integrate any upgraded sensors.

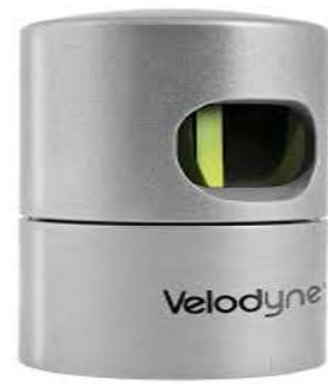

a)

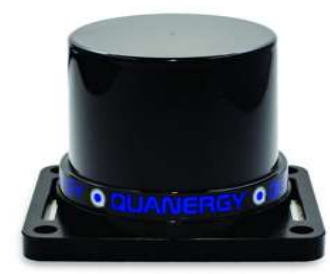

b)

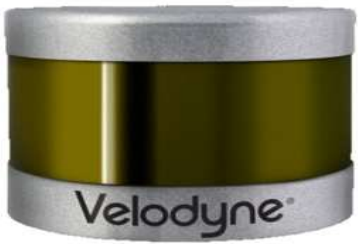

c)

Fig 3.1: Examples of SMbL scanners, a) Velodyne HDL-32E, b) Quanergy M8 and c) Velodyne VLP-16 


\subsubsection{GNSS/INS optimized selection}

In order to maintain survey-grade accuracy of the mapped environment, an accurate GNSS/INS module is essential. One of the key components of a DG 3D mapping system is the GNSS/INS module, as the positional and attitude accuracy attainable by the module has a direct impact on the accuracy of the generated point cloud. Integrated GNSS/INS modules provide a better option than separate GNSS and INS modules. The integrated module has the same timestamp for both the GNSS and the INS, thus facilitating the data fusion between the GNSS and the INS.

With regard to the optimized selection of the GNSS/INS module for the proposed mapping system, a number of GNSS/INS modules were investigated and compared. The comparative performance analysis of GNSS/INS integrated modules includes the SBG Ellipse2-D, Novatel PwrPak7D-E1, SBG Quanta UAV and Quanta UAV extra, Novatel SPAN CPT7, Novatel SPAN-IGM-A1 and Applanix APX-15 (version-3 V3). The specifications for the investigated modules are presented in Table 3.4. Images for the GNSS/INS modules are shown in Fig. 3.2. Note that all the modules utilize multi-constellation, multi-frequency GNSS receivers.

As highlighted in Table 3.4, the SPAN CPT7 provides the highest accuracy in the roll, pitch and yaw after post-processing of the data in the inertial explorer Novatel software. In addition, it features a $\pm 2.5 \mathrm{~cm}$ accuracy with the TerraStar-C pro corrections. Although its size is adequate, it has the highest power consumption and weighs $500 \mathrm{~g}$. Moreover, it is the most expensive among the investigated GNSS/INS modules. The Quanta UAV extra exhibits slightly less accurate attitude values, while only accepting RTK corrections for positions and weighing $345 \mathrm{~g}$. On the other hand, the SPAN-IGM-A1 and the PwrPak7D-E1 show a larger form-factor and an almost similar weight (510 g and $515 \mathrm{~g}$, respectively), while featuring comparable attitude accuracy values after postprocessing. The ellipse 2-D exhibits the lowest attitude accuracy of pitch and roll of $0.05^{\circ}$ and $0.2^{\circ}$ heading, while only accepting RTK corrections with $2 \mathrm{~cm}+2 \mathrm{ppm}$ positional accuracy and weighing $180 \mathrm{~g}$.

Both the Quanta UAV and the APX-15 V3 boast the best SWaP characteristics among the investigated modules, having identical attitude post-processing accuracy of $0.025^{\circ} \mathrm{pitch} / \mathrm{roll}$ with a heading accuracy of $0.08^{\circ}$. However, the APX-15 V3 has a slightly lighter weight (60 g) and a smaller form-factor. The key advantageous factor of the APX-15 V3 is its ability to directly receive the new satellite-based corrections (Trimble RTX CenterPoint with $\pm 2 \mathrm{~cm}$ accuracy). This feature boosts the practicality of the APX-15 utilization, as it lessens the need for base stations or the HW required for RTK corrections, which can be interrupted by radio or cellular network connectivity stability. Thus, the APX-15 V3 was found to be a cost-efficient practical GNSS/INS module that exhibits survey-grade accuracy with best-in-class SWaP characteristics, in addition to its ability to directly receive a satellite broadcast of the Trimble RTX CenterPoint corrections. Hence, for the present work, the selected GNSS/INS module will be the APX-15 V3 and, as mentioned earlier, the RSS will be the VLP-16. The system management unit, which is the last building block in the mapping system, is discussed in the next section. 


\begin{tabular}{|c|c|c|c|c|c|c|c|c|c|}
\hline 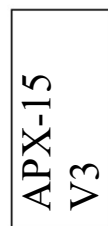 & 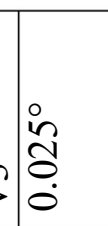 & 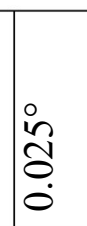 & $\mid \begin{array}{l}\infty \\
0 \\
0 \\
0\end{array}$ & 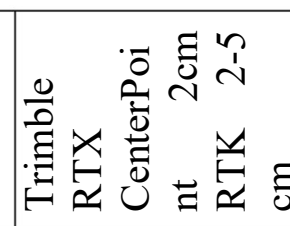 & 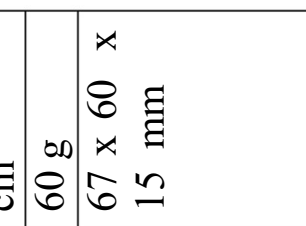 & 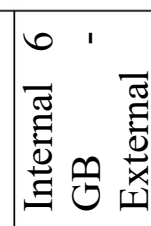 & $\begin{array}{ll}n \\
i \\
2 \\
9 \\
1 \\
0\end{array}$ & $\mid \begin{array}{ll}3 \\
3 \\
m \\
m \\
m\end{array}$ & $\begin{array}{l}3 \\
0 \\
0 \\
0 \\
0 \\
0\end{array}$ \\
\hline 究恋 & $\begin{array}{l}0 \\
\vdots \\
\vdots \\
0 \\
0\end{array}$ & $\begin{array}{l}\stackrel{0}{0} \\
0 \\
0 \\
0\end{array}$ & $\mid \begin{array}{l}0 \\
0 \\
0 \\
0\end{array}$ & 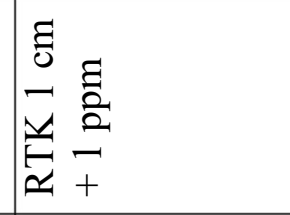 & 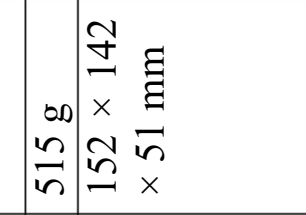 & 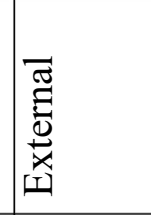 & $\begin{array}{l}n \\
2 \\
2 \\
\gamma_{0}^{1} \\
0\end{array}$ & & $\begin{array}{l}3 \\
0 \\
0 \\
0 \\
0 \\
0 \\
0\end{array}$ \\
\hline $\begin{array}{l}\frac{Z}{2}= \\
\hat{n}\end{array}$ & . & 售 & $\dot{0}$ & 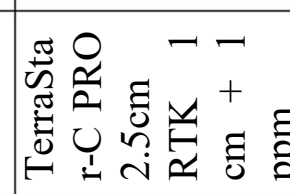 & $=$ & 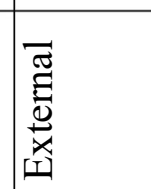 & $\begin{array}{l}n \\
0 \\
0 \\
0 \\
1 \\
1 \\
0\end{array}$ & & ${ }^{2}$ \\
\hline 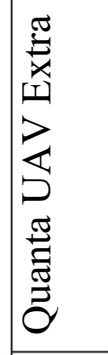 & 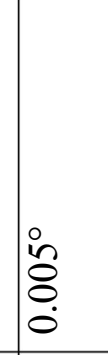 & . & $\begin{array}{l}0 \\
\tilde{a} \\
\tilde{c} \\
0 \\
0\end{array}$ & 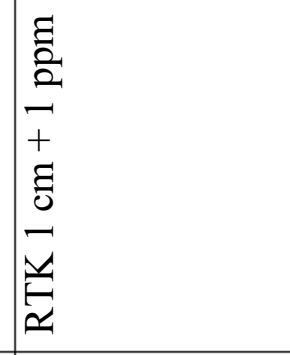 & 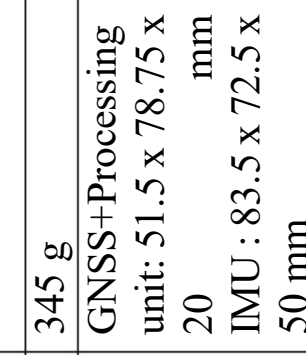 & 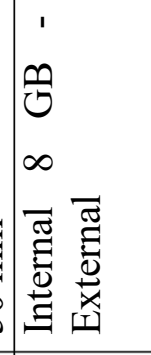 & $\begin{array}{l}0 \\
0 \\
0 \\
0 \\
0 \\
0 \\
1 \\
1 \\
\end{array}$ & $\begin{array}{l}3 \\
n \\
\text { in }\end{array}$ & $\begin{array}{l}3 \\
n \\
n \\
0 \\
m \\
n \\
n\end{array}$ \\
\hline 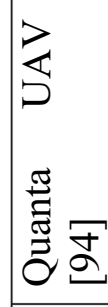 & {$\left[\begin{array}{l}i \\
0 \\
0 \\
0\end{array}\right.$} & $\begin{array}{l}\text { in } \\
0 \\
0 \\
0\end{array}$ & : & 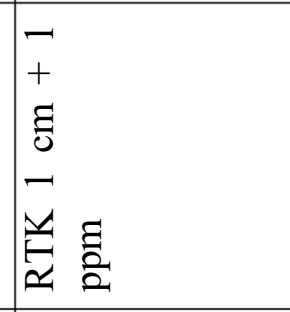 & 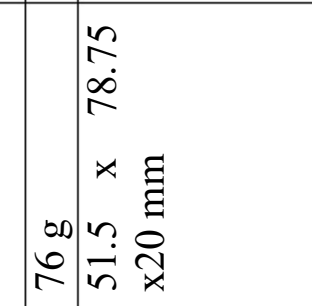 & 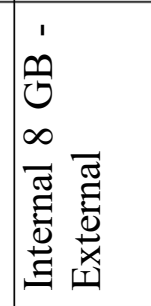 & $\begin{array}{l}0 \\
0 \\
0 \\
0 \\
0 \\
0 \\
1 \\
1 \\
1\end{array}$ & $\begin{array}{l}3 \\
n \\
n \\
m\end{array}$ & $\begin{array}{l}1 \\
n \\
n \\
0 \\
m \\
m \\
n\end{array}$ \\
\hline 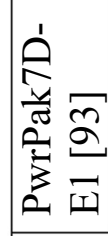 & $\begin{array}{l}1 \\
\vdots \\
0 \\
0 \\
0\end{array}$ & : & $\begin{array}{l}0 \\
\infty \\
0 \\
0 \\
0 \\
0\end{array}$ & 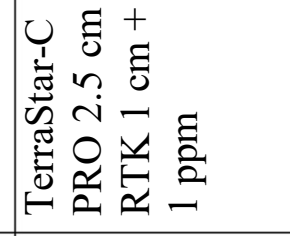 & 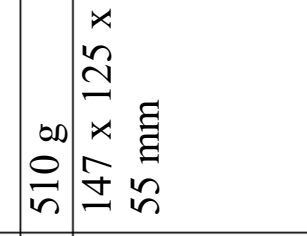 & 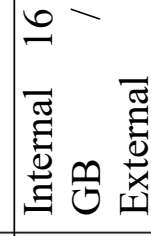 & $\begin{array}{l}0 \\
0 \\
n \\
0 \\
0 \\
9 \\
1\end{array}$ & $\begin{array}{l}3 \\
\infty \\
- \\
-\end{array}$ & $\begin{array}{l}3 \\
0 \\
0 \\
1 \\
a\end{array}$ \\
\hline 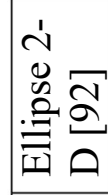 & {$\left[\begin{array}{lll}0 \\
0 \\
0 \\
0\end{array}\right.$} & $\begin{array}{l}i n \\
i \\
0 \\
0\end{array}$ & 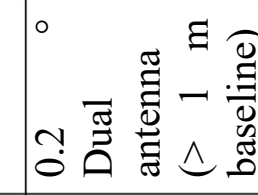 & 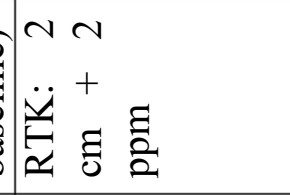 & 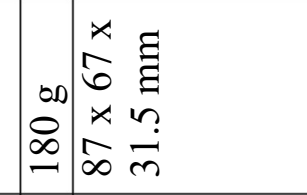 & 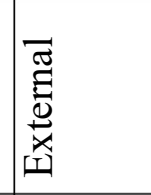 & $\begin{array}{l}n \\
0 \\
0 \\
0 \\
0 \\
0\end{array}$ & $\mid \begin{array}{lll}3 \\
n \\
-1\end{array}$ & $\begin{array}{l}3 \\
0 \\
0 \\
1 \\
a\end{array}$ \\
\hline 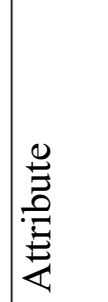 & 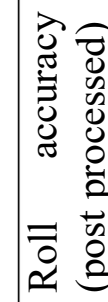 & & $\begin{array}{ll}0 \\
0\end{array}$ & 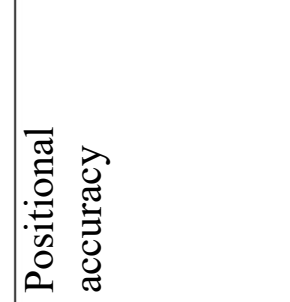 & 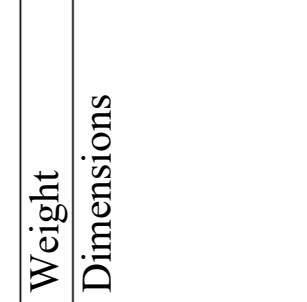 & 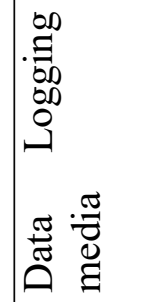 & 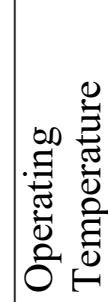 & 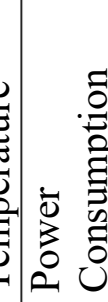 & 焉 \\
\hline
\end{tabular}




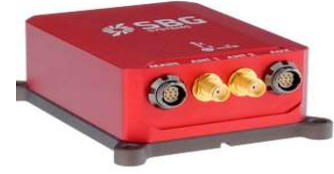

a)

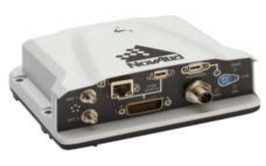

b)

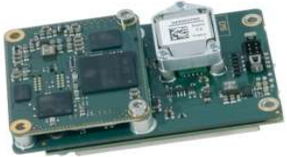

c)

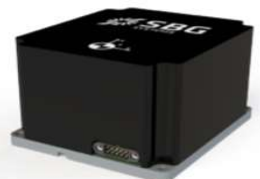

d)

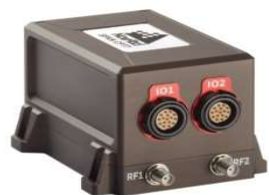

e)

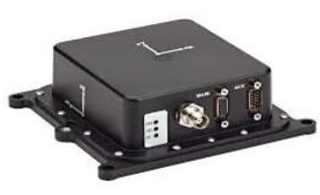

f)

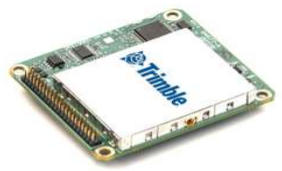

g)

Fig 3.2: Examples of GNSS/INS Modules, a) Ellipse2-D, b) PwrPak7D-E1, c) Quanta UAV

d) Quanta UAV extra, e) SPAN CPT7, f) SPAN-IGM-A1 and g) APX-15 V3 


\subsubsection{System Management Unit SMU optimized selection}

The SMU constitutes the third and final building block of the mapping system. The SMU allows for control of the mapping system and includes the power source that provides the power needed for the various system components. It also provides a means of storage for the RSS and the GNSS/INS. Minicomputers or ARM boards, such as the Raspberry-pi, were investigated as potential SMUs for the present study. The minicomputers weigh around $500 \mathrm{~g}$ and constitute almost $10 \%$ of a UAV mapping platform, like the DJI M600 payload capacity. On the other hand, although ARM boards like the Raspberry-pi weigh only $50 \mathrm{~g}$, which is much lighter than the minicomputers, they do not provide a user-friendly environment (e.g., a Windows-based minicomputer, more RAM, or advanced networking capabilities).

Thus, for the task at hand, a Windows-based minicomputer with a very small form-factor $(9.6 \mathrm{~cm}$ $\mathrm{x} 9.6 \mathrm{cmx} 1.98 \mathrm{~cm}$ ), weighing only $200 \mathrm{~g}$ and operating on just a $5 \mathrm{~V}$ power supply was selected. The minicomputer runs a Quad-Core Intel Atom x5-Z8350 processor with 2MB cache, a $2 \mathrm{~GB}$ RAM, and 64 GB of built-in storage with an expandable SD-card of up to $128 \mathrm{~GB}$. It also has a number of USB ports that support external storage devices.

The selected minicomputer features an Ethernet port that is essential to interface with the VLP-16 and receive-store its data packets. Additionally, a 3.5 inch screen and a mini wireless keyboard and mouse touchpad were selected to facilitate the practical control of the minicomputer and allow the efficient control of the mapping system as a whole. For the power supply source needed for the RSS, the GNSS/INS and the minicomputer, the power consumption from the different components was used to calculate the appropriate power supply. Two power supply sources were investigated: the anti-gravity XP-10 battery (Table 3.10 [95]) and the Talentcell rechargeable battery (Table 3.11 [96]).

Table 3.5: The XP-10 and Talentcell specifications overview

\begin{tabular}{|l|l|l|}
\hline \multicolumn{1}{|c|}{ Attribute } & \multicolumn{1}{|c|}{ XP-10 } & \multicolumn{1}{c|}{ Talentcell } \\
\hline Capacity & $18000 \mathrm{mAh}$ & $3000 \mathrm{mAh}$ \\
\hline Output voltage & $19 \mathrm{~V}+12 \mathrm{~V}+$ Two USB 5V & $12 \mathrm{~V}+\mathrm{USB} 5 \mathrm{~V}$ \\
\hline Weight & $500 \mathrm{~g}$ & $190 \mathrm{~g}$ \\
\hline Dimensions & $228.6 \times 81.3 \times 30.5 \mathrm{~mm}$ & $105 \times 63 \times 24 \mathrm{~mm}$ \\
\hline
\end{tabular}

As shown in Table 3.10, although the XP-10 provides a higher capacity $(18,000 \mathrm{mAh})$, it is still more than 2.5 times the weight of the talentcell and more than triple the size, since the components selected for the mapping systems are considered on the low-end regarding power consumption. After calculating the power demand from the mapping system's various components, it was found that the talentcell would satisfy the power demand for the mapping system and have a reduction of more than $60 \%$ of the weight and more than $70 \%$ of the size. Having a lighter weight and smaller size is crucial to satisfy stringent ULS requirements. In addition, the smaller size of the power supply adds to the flexibility of placement on the tight space within the ULS platforms. 


\subsection{Mapping system building blocks Signal Synchronization}

A precise integration of the mapping system building blocks on the HW level is essential to ensure an accurate mapping process. The HW integration is achieved by the proper wiring connections to be able to synchronize data streams from the various system components. Proper configuration between HW system components is implemented to ensure that proper signal communications are in place so that accurate synchronization can be attained. More specifically, the precise and accurate signal synchronization between the RSS and the GNSS/INS module is of utmost importance to allow for accurate 3D map production. The DG process requires accurate connection of the position and orientation data recorded by the GNSS/INS module and the laser beams fired by the RSS. In order to maintain this accurate connection, the VLP-16 and APX-15 data need to be time-stamped to the same time reference.

The APX-15 generates a sequential synchronization Pulse Per Second (PPS) signal and a NMEA \$GPRMC message. The VLP-16 then receives the PPS signal through a dedicated wire and the \$GPRMC message through a serial RS-232 interface at a baud rate of 9600. Upon signal reception and synchronization of the PPS and the \$GPRMC message, the VLP-16 data is time-stamped according to the embedded Coordinated Universal Time (UTC). In order to ensure precise synchronization, the \$GPRMC message reception should conclude no later than $500 \mathrm{~ms}$ after the rising edge of the PPS signal, as depicted in Fig. 3.3.

Subsequently, the VLP-16 time-stamped data is send to and stored on the minicomputer through the Ethernet interface, as the VLP-16 lacks an internal storage media. The measured data is sent at a rate of up to $16 \mathrm{Mbps}$. The connection between the VLP-16 and the minicomputer is secured by adjusting the corresponding link-local non-overlapping IP addresses of both the VLP-16 and the minicomputer. In the present study, an external mini Ethernet interface was utilized to simultaneously allow the monitoring of the APX-15 over a different IP local-link address.

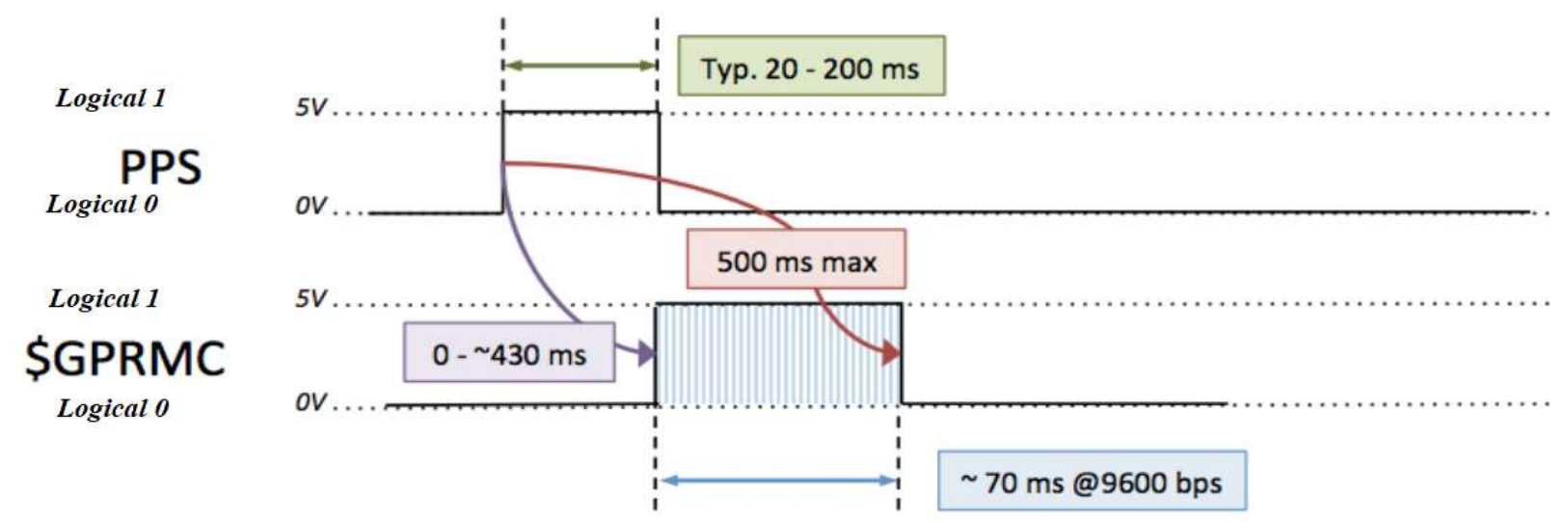

Fig 3.3: Signal Synchronization between the VLP-16 and APX-15 
Due to the significance of precise synchronization process on 3D mapping accuracy, the synchronization should be carefully monitored both in real-time and in the post-processing phase. Precise synchronization ensures that the firing time of the VLP-16 beam is accurately timestamped, thus allowing the corresponding position and orientation data to be matched and ensuring accurate mapping of the surrounding environment. The VLP-16 web UI allows the monitoring of the status of the PPS synchronization between the VLP-16 and APX-15, as shown in Fig. 3.4. Moreover, a data packets network reader, such as the Wireshark program, can be used to ensure that the \$GPRMC and PPS synchronization is valid through the position packet, as shown in Fig. 3.5.

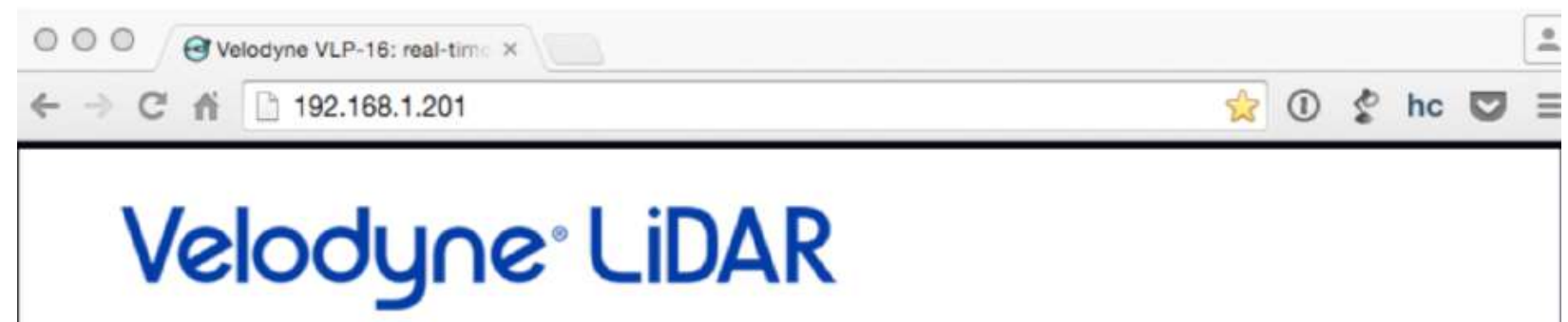

Sensor Model: VLP-16 S/N: AE02811163 MAC: 60-76-88-10-2b-9b

\section{VLP-16 USER INTERFACE}

Configuration

Laser: $\bullet$ On $\bigcirc$ Off

Return Type: Strongest $\mathrm{C}$

Motor RPM: $600+0$ Set

FOV Start: 00 End: $359+1$ Set

Phase Lock $\bigcirc$ On $\odot$ Off Offset:

Host (Destination) IP: 255.255.255.255 Data Port: $236 \xi$ Telemetry Port: $830 €$ Sef

Network (Sensor) IP: 192.168.1.201 Mask: 255.255.255.0 Gateway: 192.168.1.1

Save Configuration

Download Snapshot

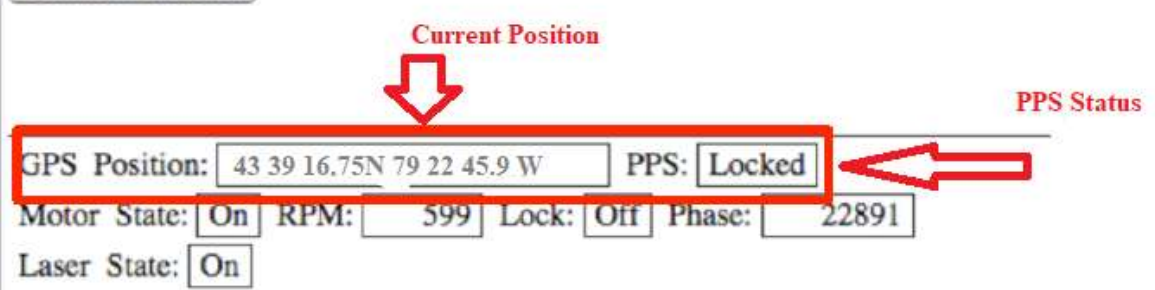

Laser State: On

Fig 3.4: Synchronization status through the VLP-16 web UI 


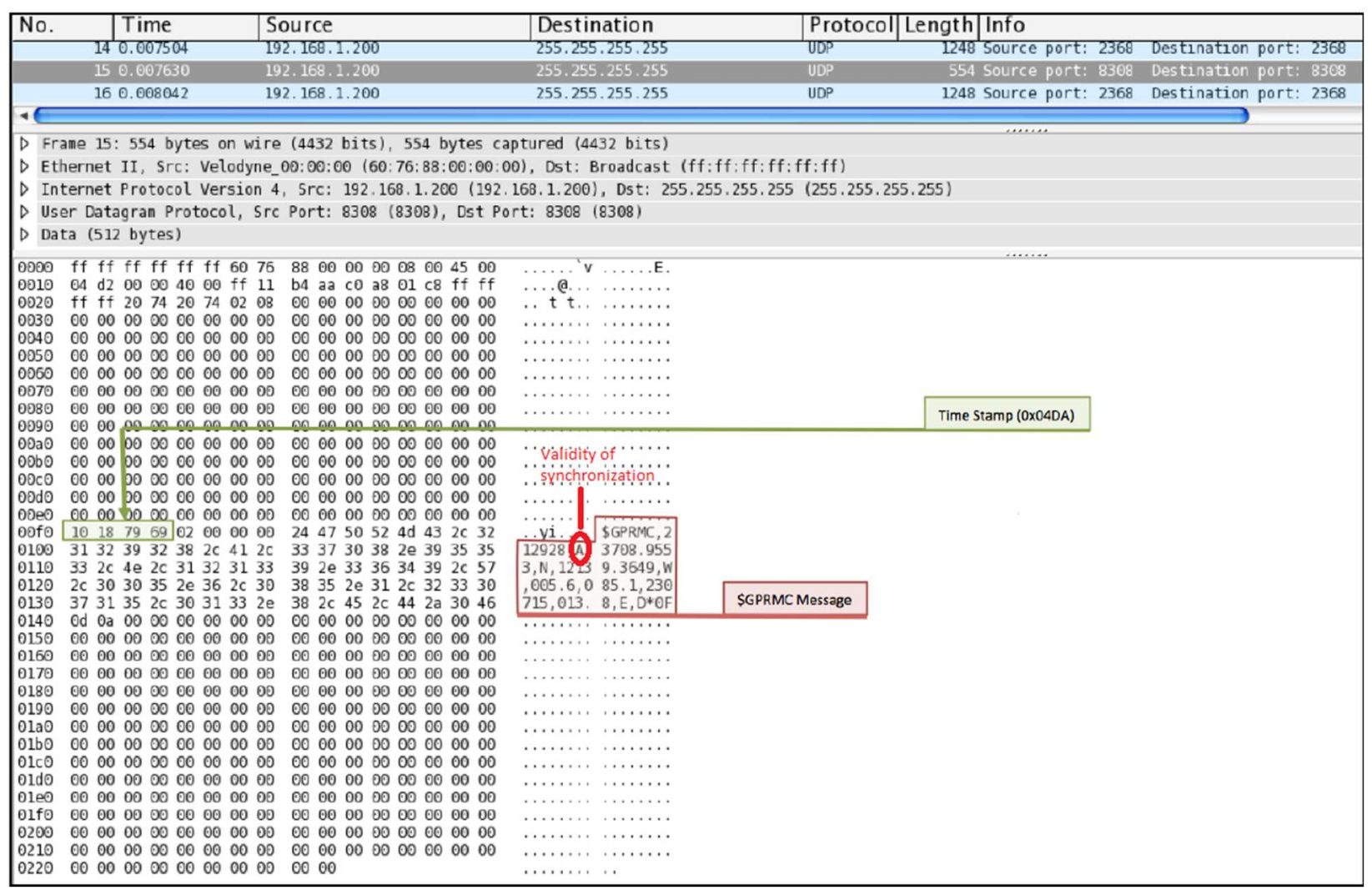

Fig 3.5: Synchronization status through Wireshark and position packet

As mentioned, precise wiring between the VLP-16 and the APX-15 circuit elements is essential to maintain the required synchronization. As experienced in the present research work, the VLP-16 pin responsible for the reception of the APX-15 PPS signal was defective by being slightly bent within the VLP-16 interface box, as shown in Fig. 3.6, causing imprecise synchronization (Fig. 3.7). After finding the cause of this imprecision, a fix was put in place by straightening the defective pin. The fix proved sufficient, as the synchronization status has been continuously monitored through the VLP-16 web UI and the position packets through the Wireshark packets reader. However, to increase the reliability of the connection, hardwiring of the APX-15 PPS signal directly to the VLP-16 circuit board has been applied.

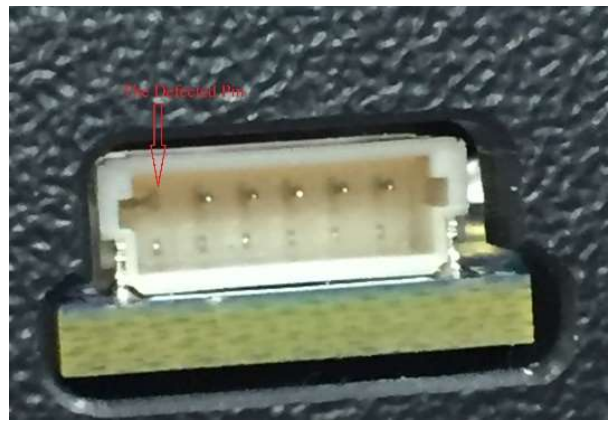

Fig 3.6: VLP-16 PPS defective pin 


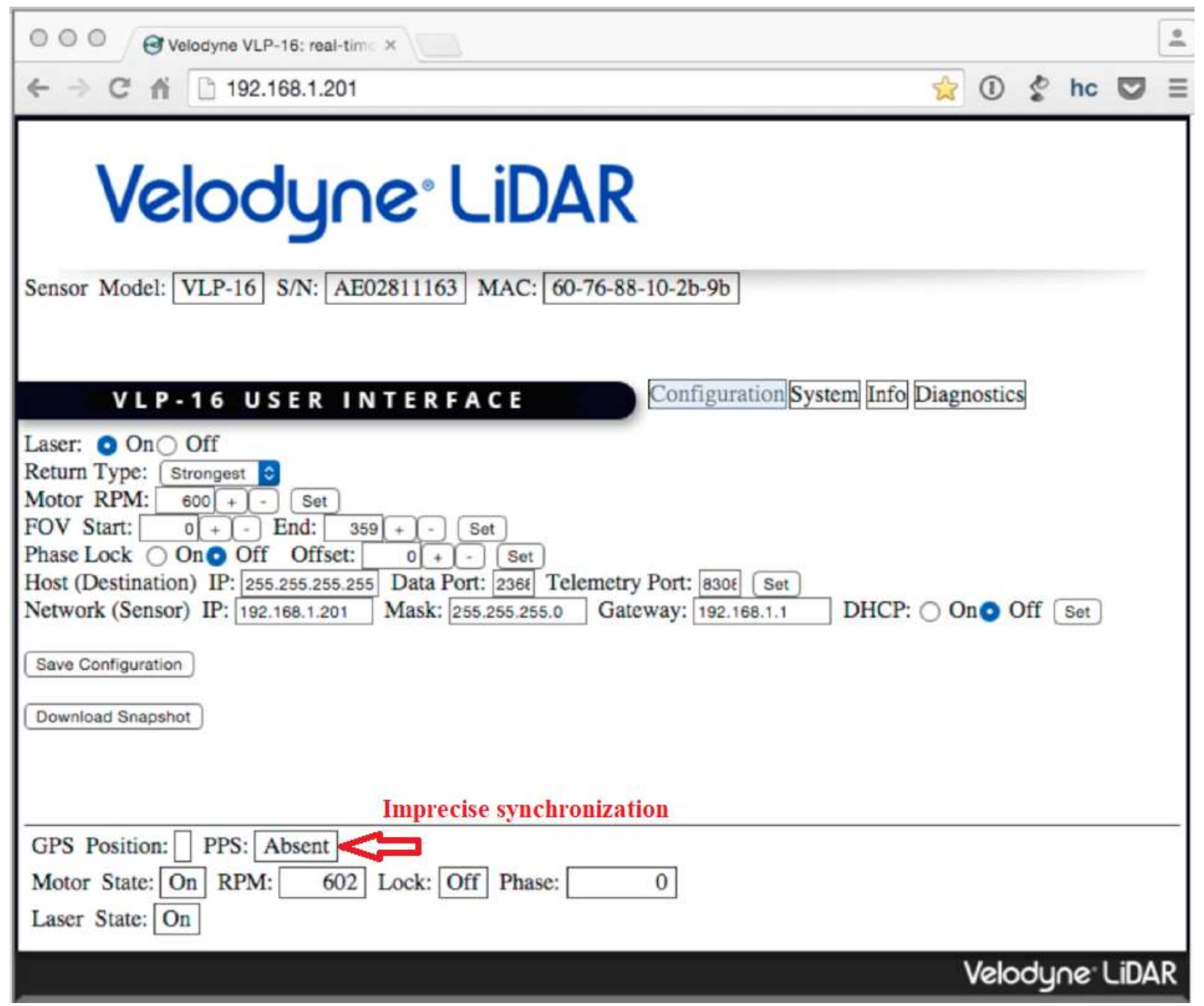

Fig 3.7: Imprecise Synchronization status

The building blocks diagram showing the signals connections, wiring and communications ports between the mapping system components is illustrated in Fig. 3.8. Note that the processing of the range data and the corresponding position and orientation data in the same time-stamp range is possible through the sensor data fusion on the SW level. 


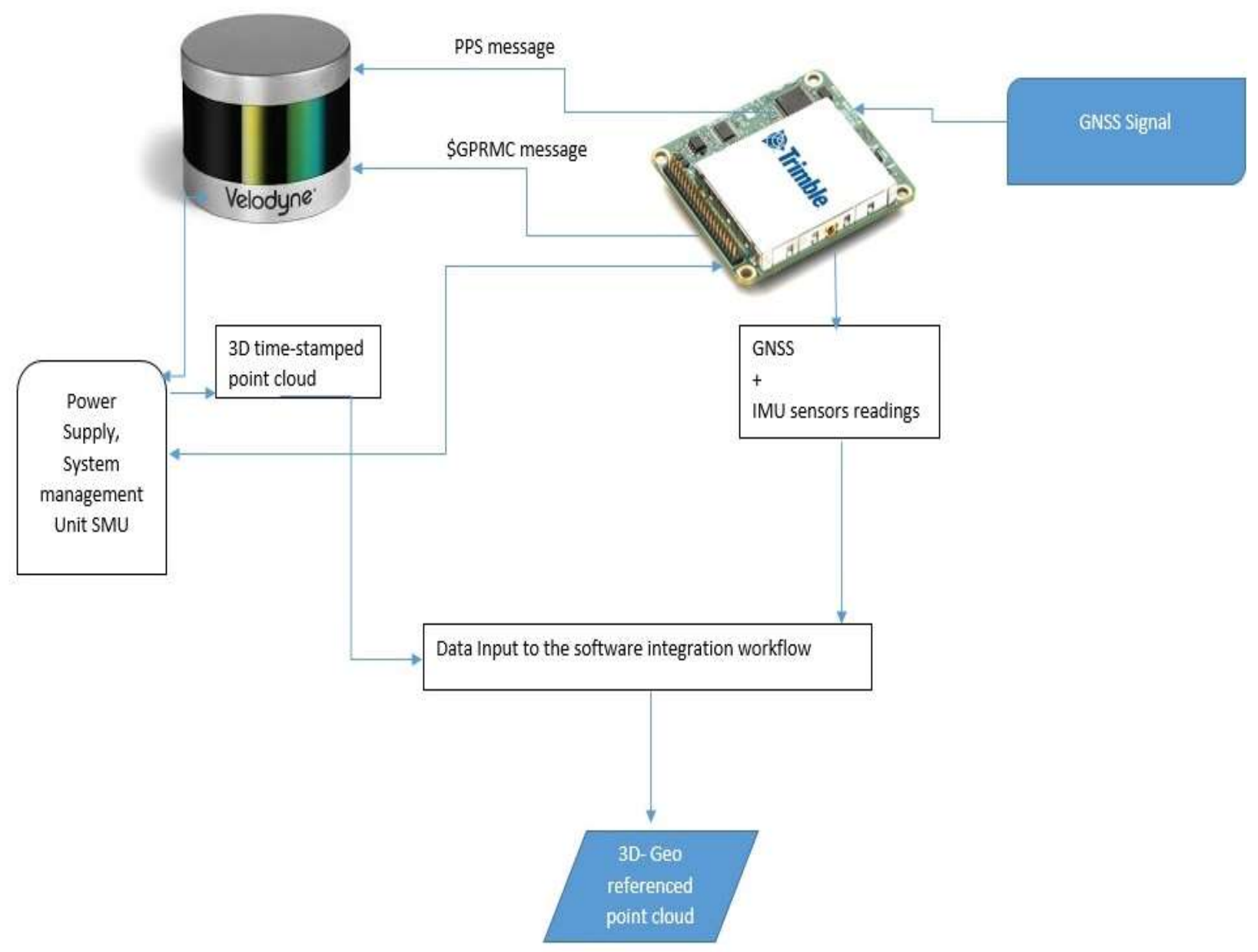

Fig 3.8: Mapping system building blocks Integration Scheme

To facilitate the deployment of the mapping system in the different data acquisitions modes, (namely, ULS, MMS and STLS), a carefully designed housing was developed, as depicted in Fig. 3.9. Moreover, smart placement of the mapping system components allowed for the minimization of the size of the mapping system while allowing for other sensors to be easily added. In addition, the smart placement enhanced the robustness of the system when mounted, as the system center of gravity is nearer to the mounting point. Additional sensors (active and/or passive) can be mounted on one of the free surfaces of the mapping system housing box. The side of the box to be used is decided upon the platform of the system (tripod, car, UAV) and the required field of view. 


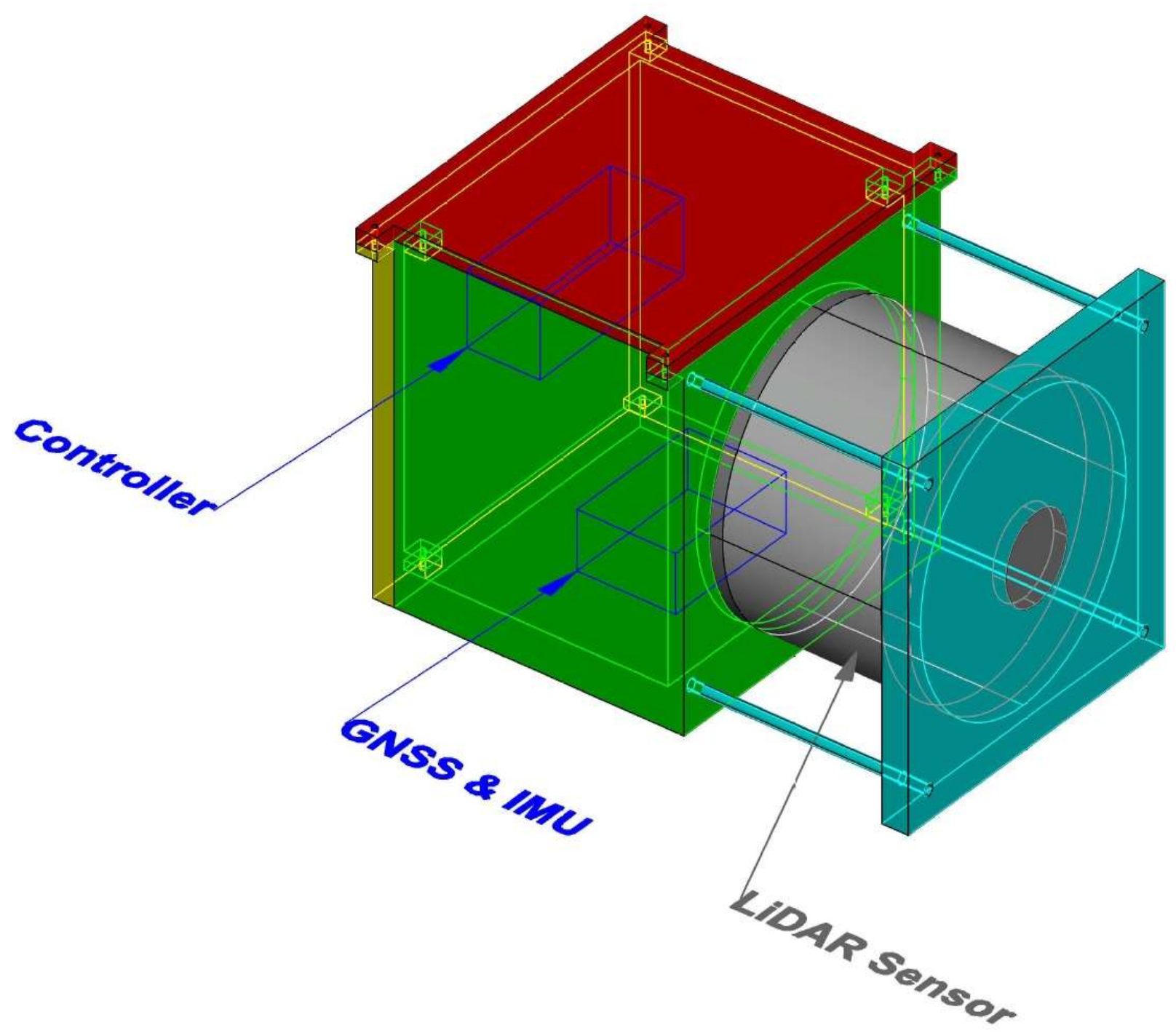

Fig 3.9: Mapping system housing

\subsubsection{Mapping system Components' EMI}

Due to the number of electronic components within the mapping system, Electromagnetic Interference (EMI) between the different electronics may occur. EMI is a disturbance caused by interactions between the electromagnetic fields generated by a circuit's elements. The GNSS antenna that receives the GNSS signals necessary for the GNSS receiver is subject to undue performance degradation caused by EMI. Furthermore, depending on the magnitude of EMI, the GNSS signals may be completely lost. The quality degradation of the received GNSS signals has a negative effect on the mapping process, as it hinders the ability to maintain an accurate position fix, which is crucial to ensure accurate $3 \mathrm{D}$ mapping. The close physical proximity of different mapping system circuit elements generally increases EMI. 
It is worth noting that EMI may be further complicated in the case of ULS mapping due to the limited space available on the UAV platform to deploy the VLP-16, the APX-15, and the SMU along with the UAV brushless motors and UAV electronics elements. In order to address the EMI challenge, precise wiring between the mapping system components must be ensured. Additionally, physically separating between the controller SMU and the GNSS antenna most often resolves the EMI issue. The housing designed to host the APX-15 and the wiring between the necessary electronic circuits may also contribute to lessening EMI. Physically separating the GNSS antenna and the other electronic components in the MMS case has been implemented by decoupling the controller SMU entity apart from the VLP-16 and the APX-15. This process greatly reduced the EMI effect on the GNSS antenna. In a ULS event, a mast may be utilized to elevate the GNSS antenna to a higher level than the UAV platform electronic components, motors, and mapping system elements.

\subsection{Mapping System Building Block Data Fusion}

The integration between the system's different components involved both hardware and software. The SW integration follows the HW integration implementation, as described in section 3.3. One of the main challenges of processing the data captured by a mapping system is the huge amount of data captured (i.e., hundreds of millions or even billions of points). Furthermore, as these data require processing, efficient data processing workflows are essential to ensure a reasonable turnaround time of the 3D map.

The technological advances in the big data processing realm such as multi-threading, parallel processing, and cloud computing allow for the efficient utilization of different HW resources to expedite the processing task of the mapping system's captured data. The proper workflows for the fusion of the multimodal sensor data were designed to be cloud-computing- and parallel processing-ready. Dedicated algorithms were also developed for the sensor data fusion on the SW level. The coding of the developed algorithms followed the vectorization scheme. Vectorization is an optimized method of coding which allows the utilization of the ability of modern CPUs to apply a single instruction to multiple data SIMD, thus leading to orders of magnitude performance gains. The main steps in the SW integration workflow are summarized as follows:

1- $\quad$ APX-15 GNSS and IMU integration

2- $\quad$ Processing and preparation of step 1, with output to be used in step 3

3- $\quad$ Integration of step 2 output (adjusted smoothed trajectory) and VLP-16

\subsubsection{Data processing of GNSS and IMU data}

In regard of the system integration on the SW level, there are three data streams involved. The first is the remote sensing sensor data stream (i.e., VLP-16), denoted here by $\left(\mathrm{S}_{1}\right)$. The second and third data streams are the two APX-15 entities: the GNSS sensor data stream $\left(\mathrm{S}_{2}\right)$, and the IMU sensor data stream $\left(\mathrm{S}_{3}\right)$. The GNSS data stream is characterized by a low update rate, but without suffering data drift. The IMU data stream, on the other hand, is characterized by a high update rate and highly accurate IMU calculated values; however, with time, it suffers data drift. GNSS readings may be used to constrain this drift.

The integration of GNSS and IMU data streams is achieved by using a Kalman filter algorithm. The forward and backward data smoothing is then applied to enhance the accuracy of the 
integration process, along with Zero velocity UPdaTes (ZUPT). These are points where the system is not moving and are used to enhance the calculated position. Note that the APX-15 is equipped with the proper sensors that can receive real-time GNSS corrections, whether satellite-based corrections or base station corrections embedded in the GNSS/IMU sensor type selected. The corrections can be included either directly in real-time or in post-processing, as the mapping system architecture enables this flexibility. The output from the second and third data streams, denoted here as $\left(\mathrm{S}_{2_{-}}\right)$, provide highly accurate trajectory and orientation values that can be integrated with the first data stream $\left(\mathrm{S}_{1}\right)$. A UTC timestamp is used to link $\left(\mathrm{S}_{2_{-} 3}\right)$ and $\left(\mathrm{S}_{1}\right)$.

\subsubsection{Preparation of LIDAR data}

The workflow developed for (S1) data preparation allows for the full control of the amount of data needed to be analyzed. This is achieved by having full access to each individual frame or sweep of the sensor in the post-processing phase. Applying the workflow this way has the following advantages:

i) More control is added to the operator to include the key frames or the convenient frame rate that is needed.

ii) Parallel processing for data analysis is allowed, thus expediting the analysis process.

iii) The SW workflow allows for cloud computation to be easily applied.

iv) The designed workflow provides a dramatic decrease of the time required for the turnaround of the final product while still having control up to the single sweep level, thus increasing the efficiency and productivity of the mapping process.

\subsubsection{Interpolation and joining with synchronized time-stamps}

Since S1, S2 and S3 data streams have different frequency, a time-step interval is chosen to interpolate the lower frequency (S2_3), which is then matched and joined with the higher (S1) one. Since the APX-15 data (S2_3) update rate is much lower than the VLP-16 collection rate (S1), the interpolation of S2_3 is indispensable, depending on the rate of change of the position or the orientation the time-step interval changes. The higher the rate of change of the system position or orientation, the smaller the time-step used for the interpolation. For example, the higher the vehicle speed, the smaller the interpolation step needed.

The developed code is parallel-computing-ready and provides the operator with full control over each individual sensor frame or sweep. Extracting each sweep and doing the processing for it enables the efficient usage of the computer resources and also allows the usage of multiple computers to process different parts of the data. In addition, to increase the user-friendly interaction environment for diverse end-users, the processing applied on each sweep can be checked and can exactly follow which frames are currently processed. Moreover, the algorithms developed enable the utilization of the parts of the data that are already processed while the other parts are still in process.

Additionally, the vectorization of the operations implemented within the developed code enhance the processing time required for (S2_3) and (S1) data stream integration. Instead of using the conventional loops to run the code on the data, each column of the data is treated as a vector and the processing run on the complete vector, thus considerably decreasing the processing time. A 
flowchart summarizing the workflow for the sensor data fusion is illustrated in Fig. 3.10. Note that the accurate matching between (S2_3) and (S1) data streams is an indispensable prerequisite for the direct georeferencing DG process that is discussed in more detail in the next chapter. 


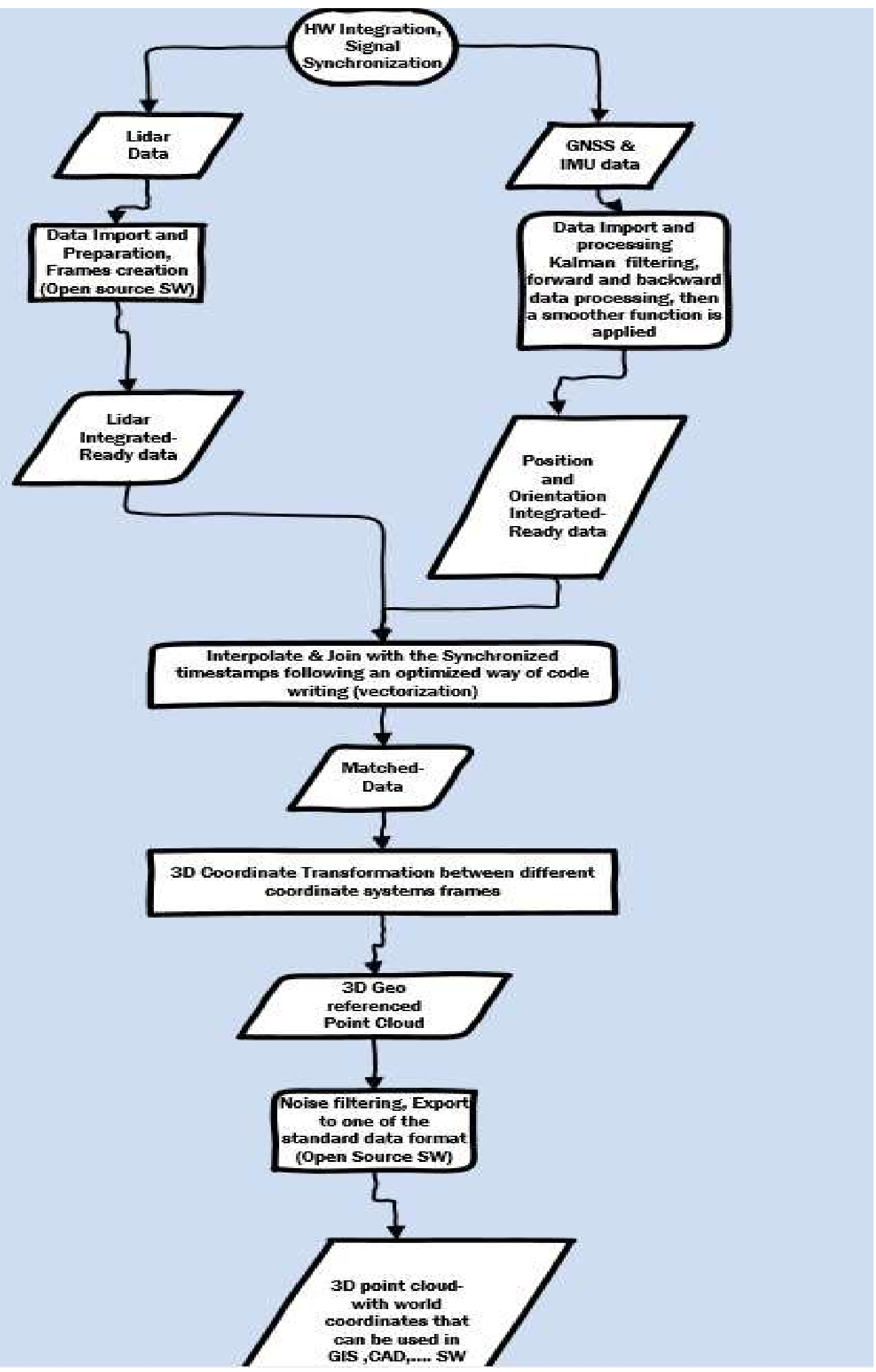

Fig 3.10: Workflow for the sensor data fusion 


\section{THE MAPPING SYSTEM'S DIFFERENT MODES OF OPERATION AND THE UNCERTAINTY PREDICTIVE MODEL}

\subsection{Overview}

As highlighted in the preceding chapters, a key objective of this dissertation is the development of a platform-independent, plug-n-go LiDAR-based mapping system that can be utilized in different mapping modes, namely STLS, MMS, and ULS. The selected LiDAR RSS - the VLP-16 - is a state-of-the-art sensor of this type. However, this option comes with a number of challenges, including a relatively less dense measured point cloud, a limited Vertical Field of View (VFOV), and a coarse vertical resolution. These challenges are further accentuated in the STLS mode, as the sensor is originally developed for the kinematic autonomous vehicle domain.

In order to overcome the aforementioned VLP-16 drawbacks and to increase the potential usage of the sensor, a thorough study of the sensor data capturing mechanism is conducted. A new VLP16 data capturing scheme is introduced to enable the usage of the mapping system in the STLS mode. Moreover, the assessment of the usage of a low-cost Motion Capture (MoCap) sensor ( 250 US\$) is carried out. The new data capture scheme is tested in few different representative environments, namely a building façade, and an outdoor area in a backpack platform mode. The new scheme proved successful in increasing the potential usages of the sensor and overcoming its drawbacks.

In addition, the usage of the mapping system in the MMS and the ULS is performed. Lastly, the rigorous analysis for the system error budget is developed. Detailed discussions and presentation are provided in the sections ahead.

\subsection{VLP-16 Data Capture Mechanism}

The VLP-16 consists of 16 pairs of emitters and receivers for 16 continuously-fired laser beams with fixed orientation within the sensor. The 16 beams spin around the sensor's internal spinning axis to cover $360^{\circ}$ at a customizable spinning rate of 5-20 HZ. The vertical angle of each laser beam is fixed and can be retrieved by the laser beam ID. The reload and firing of the 16 laser beams takes $55 \mu \mathrm{s}$, thus enabling the firing of 300,000 pts/sec. Figure 4.1 depicts the 16 laser beams, while Table 4.1 presents the fixed vertical angle for each beam. 


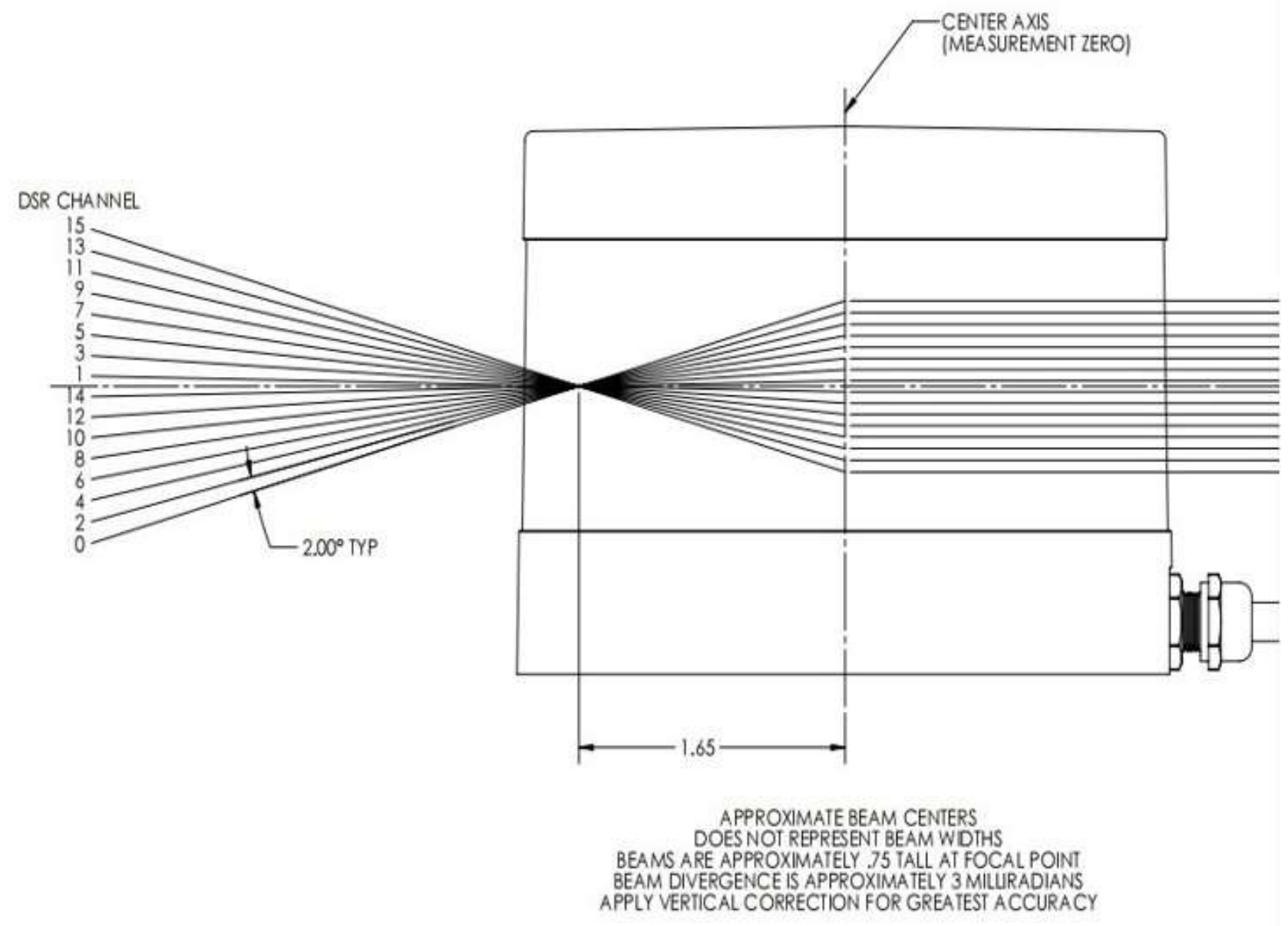

Fig 4.1: VLP-16 Laser Beams

[VLP-16 Manual] 
Table 4.1: VLP-16 Laser Beams Vertical Angles

[VLP-16 Manual]

\begin{tabular}{|c|c|}
\hline Laser ID & Vertical Angle \\
\hline 0 & -15 \\
\hline 1 & 1 \\
\hline 2 & -13 \\
\hline 3 & 3 \\
\hline 4 & -11 \\
\hline 5 & 5 \\
\hline 6 & -9 \\
\hline 7 & 7 \\
\hline 8 & -7 \\
\hline 9 & 9 \\
\hline 10 & -5 \\
\hline 11 & 11 \\
\hline 12 & -3 \\
\hline 13 & 13 \\
\hline 14 & -1 \\
\hline & 15 \\
\hline
\end{tabular}

As shown in Fig. 4.1 and Table 4.1, the beams below the sensor horizon are numbered as even numbers, starting with the Laser ID of zero as the furthest beam, with a vertical angle of $-15^{\circ}$. The beams above the sensor horizon are numbered as odd numbers and have positive vertical angles, with beam number 15 being the furthest beam, with a vertical angle of $15^{\circ}$. Thus the VFOV of the sensor is $\pm 15^{\circ}$, which is sufficient for the original usage of the sensor for the autonomous vehicles but the utilization of the sensor for 3D mapping will suffer from such a limited VFOV. Moreover, the vertical coarse resolution $(\phi)$ of $2^{\circ}$ between the laser beams results in vertical scan gaps $(\psi)$ in the scanned environment that can reach $3.5 \mathrm{~m}$ at the sensor max scanning range $(\rho)$ of $100 \mathrm{~m}$. The vertical scan gap $(\psi)$ is directly proportional to the sensor scanning range $(\rho)$ and vertical coarse resolution $(\phi)$. The relation between the $\psi, \phi$ and $\rho$ is expressed in Equation 4.1.

$$
\psi=2 \rho \sin (\phi / 2)
$$

Where

$\phi$ is the vertical scan gap

$\rho$ is the range to the target

$\psi$ is the vertical resolution 
The VLP-16 uses spherical coordinates in referencing the scanned environment to the sensor 3D coordinates frame. The VLP-16 3D coordinate frame axes - horizontal angle $(\alpha)$ and vertical angle $(\omega)-$ are shown in Fig. 4.2. As can be seen in the figure, the Y-axis is defined along the sensor cable direction going forward, the $\mathrm{Z}$-axis is defined upwards, and the $\mathrm{X}$-axis direction completes a right-hand $3 \mathrm{D}$ coordinate system. The horizontal angle $(\alpha)$ is reported in the transmitted data packet to the system management unit every other firing, while the vertical angle is fixed for each laser beam and can be retrieved through the laser beam ID, which is also transmitted through the data packets.
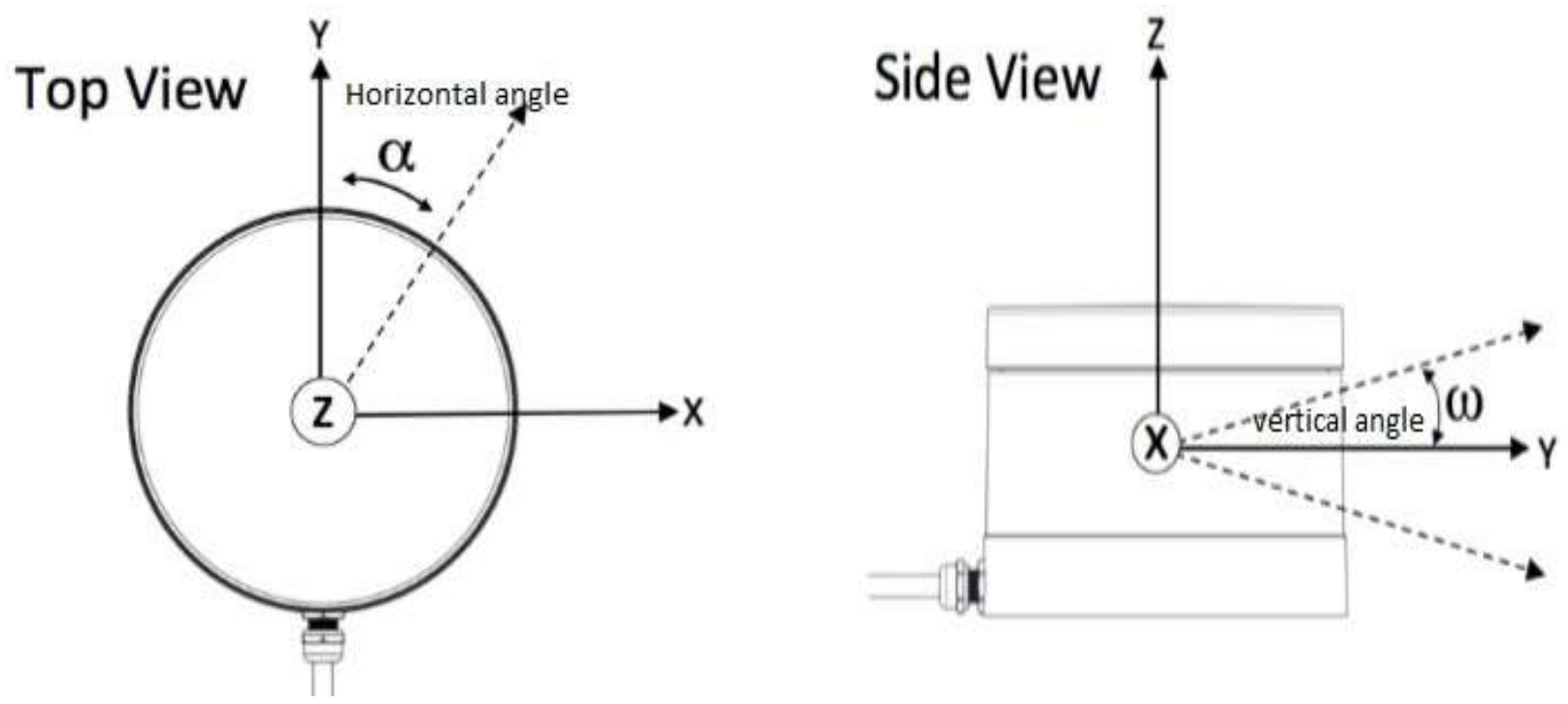

Fig 4.2: VLP-16 Axes; Horizontal and Vertical angles [VLP-16 Manual]

The measured point 3D Cartesian coordinate referenced to the sensor frame can be calculated according to Equation 4.2.

Where

$$
\left[\begin{array}{l}
x \\
y \\
z
\end{array}\right]=\rho\left[\begin{array}{c}
\cos (\omega) \sin (\alpha) \\
\cos (\omega) \cos (\alpha) \\
\sin (\omega)
\end{array}\right]
$$

$\omega$ is the vertical angle

$\rho$ is the range to the target

$\alpha$ is the horizontal angle 
As discussed and highlighted in Chapter 3, the 3D coordinates of the measured points which are time-stamped and synchronized can be matched with the GNSS and IMU data streams to produce a 3D georeferenced point cloud.

\subsection{Modes of Operation}

In order to overcome the limited VFOV and coarse vertical resolution, especially in the stationary mode of operation, a new mapping scheme is proposed. The system-positioning components (the position GNSS and orientation IMU system [POS]) are mounted in the housing and attached to the laser sensor in such a way that the possibility of having a gimbal lock is minimized. Three different angles can describe the rotational motion of the mapping system components, as shown in Fig. 4.3.

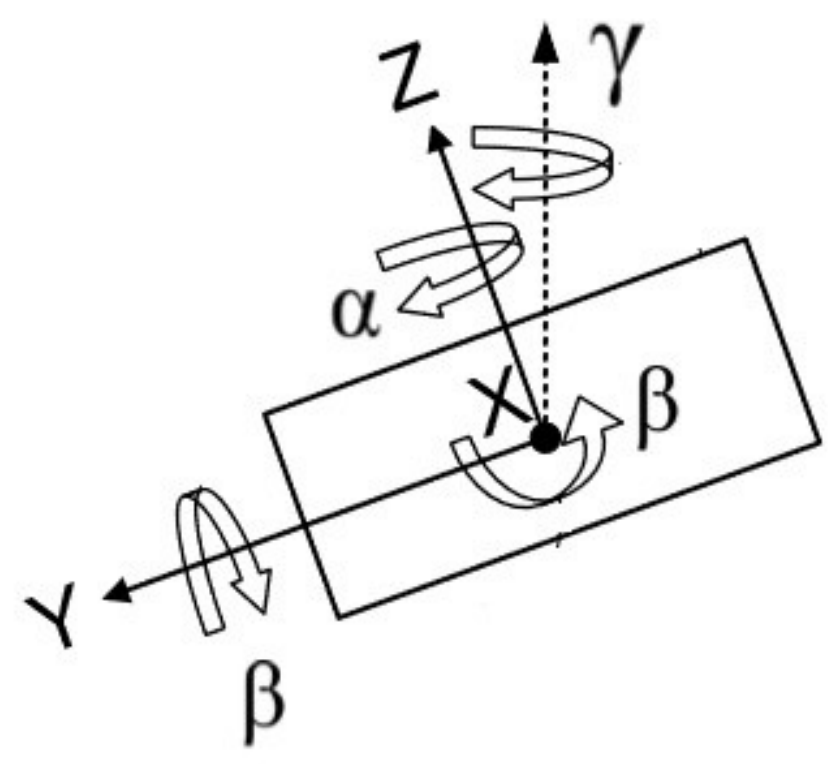

Fig 4.3: The system rotational motion angles

$\alpha$ is the laser sensor internal rotation angle

$\beta$ is a vertical rotation angle about $\mathrm{x}$-axis or $\mathrm{y}$-axis

$\gamma$ is a horizontal rotation angle about the vertical

The three sensor orientation angles present three degrees of freedom around the sensor. Angle $\alpha$ is the internal rotation angle around the LiDAR sensor spinning axis; angle $\beta$ is a vertical angle about $\mathrm{X}$ - or Y-axis; and angle $\gamma$ is a horizontal angle about the vertical. Both $\beta$ and $\gamma$ can be adjusted manually or automatically to fill the sensor multi-beam scanline gaps, increase the system field of view, and densify the point cloud. As well, $\beta$ and/or $\gamma$ can be adjusted according to the platform moving direction, the environment to be scanned, and the field of view directions, i.e., horizontal or vertical field of view (HFOV or VFOV)). This physical rotation of the system as a whole allows the efficient usage of the system for the three different scanning setups: 
1- $\quad$ Stationary Setup (STLS)

2- $\quad$ Mobile Setup (MMS)

3- $\quad$ Airborne (UAV) Setup (ALS)

\subsubsection{Stationary mapping Setup}

In stationary mode, the system is mounted in a fixed position, e.g., tripod. The physical setup allows for either manual or automated rotation. The multi-beam spinning LiDAR sensor (VLP-16) can be mounted with its spinning axis almost horizontal. The two components (the LIDAR and the POS) then rotate manually or automatically around the vertical axis, as shown in Fig. 4.4(b). In this way, the system has the widest possible coverage area ever, providing $360 \mathrm{HFOV}$ and 360 VFOV. In addition, the gaps between the scan lines are greatly eliminated, thus densifying the point cloud coverage. This is achieved by filling the coarse vertical resolution between the scanning lines with the rotation of the system as a whole.

Another configuration, the multi-beam spinning LiDAR sensor, can also be mounted with its spinning axis almost vertical. The two components (the LiDAR and the POS) then rotate manually or automatically around the $x$-axis or $y$-axis $(\beta)$, as depicted in Fig. 4.4(a). 




Fig 4.4: System Operation Method - Stationary Setup

It is worth noting that for the rotation process to be automated, a step-motor has been selected after a thorough comparison of multiple motor types. The selected motor is ultra-compact and lightweight, and its maximum payload exceeds the mapping system weight. Moreover, the motor is low-cost ( 200 US\$) and weighs only $245 \mathrm{~g}$. The reasonable cost and light weight add to the ease and practicality of the new mapping scheme and can also be utilized if needed in the ULS mode as well, since it meets the stringent UAV SWAP requirements. In addition, the motor can be controlled wirelessly using an iOS or Android app, further enhancing the versatility of the system usage and user-friendliness of the system operation. 


\subsubsection{Mobile mapping Setup}

The system may also be used in mobile mode, mounted on a vehicle or any other moving platform e.g., vehicle, backpack, etc. Unlike conventional mapping systems, which usually require specific characteristics for the vehicle the mapping system is mounted to (making it difficult to move the system from one vehicle type to another), the developed system's light weight and small formfactor add to the versatility and practicality usage of the system on any vehicle. A number of coupling mechanism to mount the developed system to the moving platform were studied, and a simple yet effective coupling mechanism has been chosen. A lightweight, compact, 3-point suction-cup mount has been selected to be used as the coupling mount of the developed system to any vehicle. The system's light weight and small form factor adds flexibility to the mounting location on the vehicle, enabling it to be mounted anywhere, whereas earlier MMS had to be mounted on the car roof. Moreover, the system can be used as a handheld device, on a backpack setup, or even attached to a person's waist belt. The light weight and small form-factor is achieved by opting for the smallest and lightest option for each component of the system, along with a smart placement scheme.

The rotation angle used to physically rotate the sensor around its axis does not need be changed, as the laser beams by default spin around the axis. Any initial orientation alignment of the system can be used for angle $\beta$, as shown in Figs. 4.5 (a), (b), (c). The sensor can also rotate around the vertical $(\gamma)$, as illustrated in Fig. 4.5 (d). The new mapping scheme that utilizes the new $\beta$ and/or $\gamma$ automated rotation would be available to be used in the mobile mapping mode as well, although the added advantage is less obvious than in the stationary mode case. 


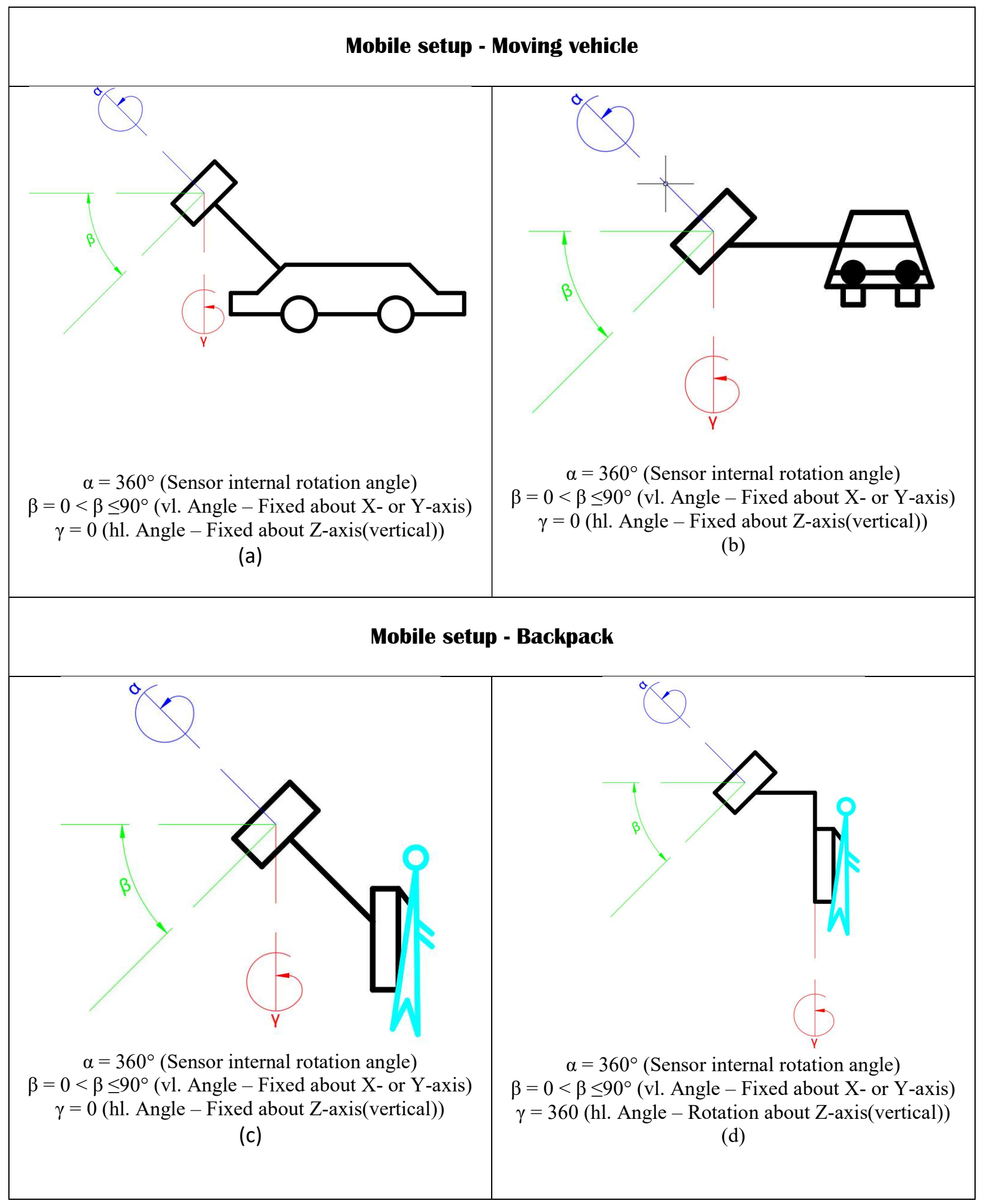

Fig 4.5: System Operation Method - Mobile Setup 


\subsubsection{UAV mapping Setup}

In the UAV-mapping mode, the system can be mounted on any UAV (subject to the payload capacity) or a plane. The system's light weight (less than $2 \mathrm{~kg}$ ) and small form-factor allow it to be mounted on small-sized UAVs with a payload capacity of more than $2 \mathrm{~kg}$. In the airborne mode, the rotation angle used to rotate the sensor physically around its spinning axis does not need be changed, as the laser beams, by default, spin around this axis. Any initial orientation alignment of the system can be used, as shown in Fig. 4.6. In a drone-based airborne mapping application, the ability to rotate the range sensor along these new rotation angles $\beta$ and/or $\gamma$ provides an expanded field-of-view to enable range data to be collected over a larger area when the drone is stationary. In contrast, a drone with a conventional sensor system needs a larger flight path to collect the same amount of range data. Thus, the new system provides more efficient ways to do UAV mapping and inspection.

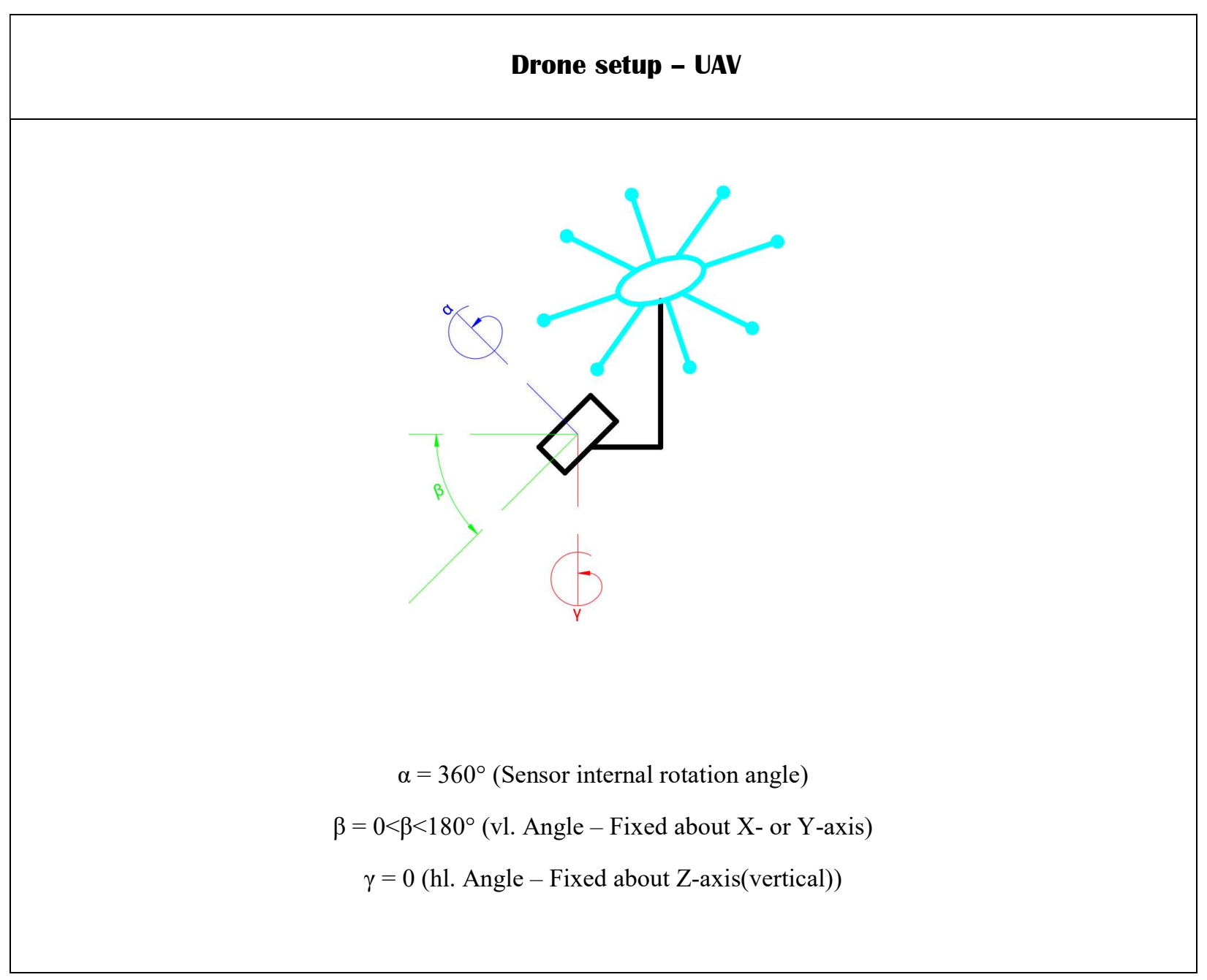

Fig 4.6: System Operation Method - UAV Setup 
It is worth noting that in typical UAV-mapping mode, the VLP-16 is mounted with its spinning axis almost horizontal and along the UAV flying direction. This allows for better resolution and less point spacing in the cross-track direction, while the UAV movement itself will enable the filling of scanline gaps along the track.

\subsection{System Uncertainty Predictive Model}

The quality of the scans obtained depends on a number of factors. These include LiDAR sensor capabilities, data acquisition mode, scanned environment, target reflectivity and the algorithms used to stich multiple scans together. The developed mapping system consists of a number of multi-modal sensors, each with a different level of precision, adding to the complexity of LiDARbased mapping as well as to the challenge involved in the verification and validation of the output products [97]. The output mapping accuracy of the system depends on the precision of the RSS and POS. A rigorous error propagation analysis is carried out in order to calculate the uncertainty attached with the utilization of the new developed system. In order to do the error propagation analysis, the georeferencing process is first explained, as follows.

The georeferencing process of the point cloud involves transforming the various sensor data sets from different Coordinate System (CS) frames to a common mapping coordinate system frame that uses real-world coordinates. The interpolated and matched data from the VLP-16 data stream and the APX-15 data streams have different coordinate system frames. The multi-sensor mapping system includes an IMU body CS frame, a GNSS CS frame ECEF, a local-level or navigational CS frame, a vehicle CS frame, and a (range) sensor CS frame. The IMU body CS frame can be used as a reference CS frame to which the other CS frames are related. For example, each point of the range data can be transformed to have the corresponding position and orientation of the orientation sensor through the boresight angles and level arms.

Figure 4.7 provides a visual representation of the different CS, boresight angles (e.g., relative orientation) and lever arms (e.g., offsets) between the different coordinate system frames used in the multi-sensor mapping system. The transformation may begin by detecting the relation between the IMU body frame and the North East Down (NED) local frame. Alternatively, two right-handed variants may be used in alternative implementations: East North Up (ENU) coordinates or North East Down (NED) coordinates. The boresight angles and lever arms between the IMU body frame and the sensor frame, and the boresight angles and lever arms between the sensor frame and the vehicle frame are used to relate the sensor frame and the vehicle frame with the local frame. The GNSS ECEF frame can be transformed to the local frame and used to aid in the pre-processing of the orientation data. The data processing unit continuously maps the relation between the different CS frames and combine the location and orientation data to map the trajectory and orientation of the range sensor with reference to the local frame. 


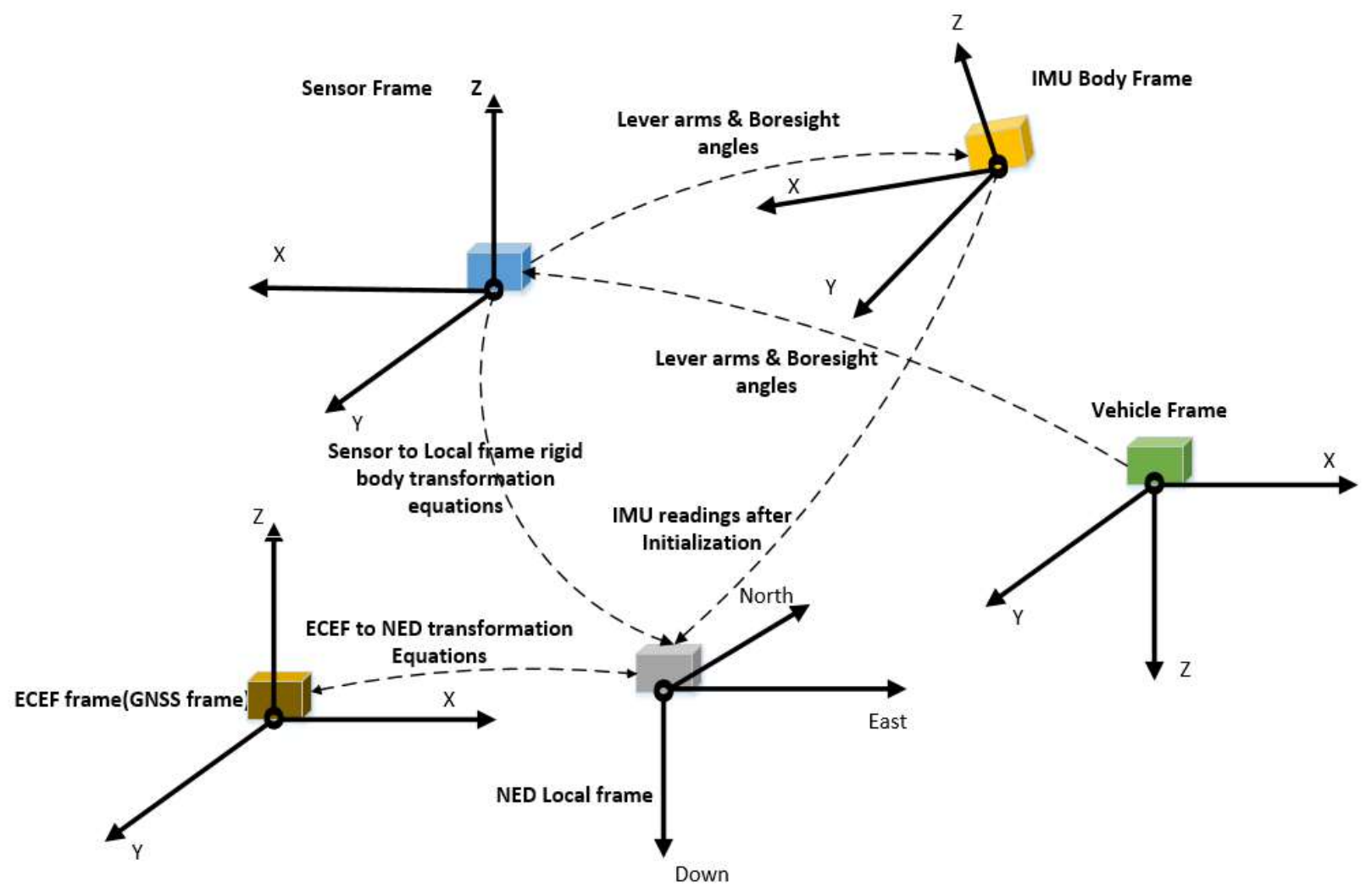

Fig 4.7: Different CS, Boresight angles and lever arms

The three data streams, i.e., the IMU (orientation) data, the GNSS (position/location) data and the range data, are matched by first performing signal synchronization between the POS (APX-15) and the range sensor (VLP-16). This is done through the PPS and the \$GPRMC message to obtain time-stamped range data that is on the same time-reference of the POS data. The GNSS and the IMU data may then be combined to obtain enhanced trajectory (i.e., positions) and orientation data. The enhanced trajectory and orientation data is then interpolated, as described previously, and matched to the range data based on finding the same time-stamps in the enhanced trajectory, orientation data, and range data. As a final step, the transformed trajectory and orientation data from APX-15 with the time-stamped range data from the VLP-16 are combined to generate a 3D geo-referenced point cloud data. At this point, the range data can be georeferenced using realworld mapping coordinates.

Thus, the relation between the RSS, POS measurements and the georeferenced point cloud can be expressed through the direct georeferencing DG mathematical model. The LiDAR-based DG mathematical model can be expressed as a functional model through Equation 4.3 and a stochastic model that is presented through Equation 4.4. The detailed derivation of the uncertainty predictive model is provided in Appendix A. 


$$
P_{l l}=r_{l l}(t)+R_{b}^{l l}(t) \cdot\left(R_{s}^{b} \cdot P_{s}(t)+l_{s}^{b}\right)
$$

Where

$P_{l l}$ is the $3 \mathrm{D}$ coordinates of the point in the local level frame

$r_{l l}(t)$ is the time dependant $3 \mathrm{D}$ coordinates of the body frame origin in the local level frame

$R_{b}^{l l}(t)$ is the time dependant rotation matrix from the body frame to the local level frame

(Defined through the time dependant roll, pitch, and yaw)

$R_{S}^{b}$ is the boresight rotation matrix between the body frame and the sensor frame

$P_{S}$ is the $3 \mathrm{D}$ point coordinates in the sensor frame

$l_{s}^{b}$ is the lever arm between the sensor CS frame origin and the body frame origin

While the stochastic part of the LiDAR-based DG mathematical model can be expressed through the law of error propagation, the uncertainty in the 3D coordinates of the georeferenced point cloud is related to the precision of the underlying VLP-16 and APX-15 measurements in addition to the precision in boresight angles and lever arms determination. Equation 4.4 presents the stochastic part of the model.

$$
C_{\text {sys }}=J C_{\text {par }} J^{T}
$$

Where

$C_{s y s}$ is the variance-covariance matrix of the georeferenced 3D coordinates;

It is a $[3 * 3]$ matrix

$C_{\text {par }}$ is the variance-covariance matrix of the random variables in the DG Equation (the POS position, POS attitudes, range, scan angles, boresight angles, lever arms); it is a $\left[15^{*} 15\right]$ matrix

$J \quad$ is the Jacobian matrix; the partial derivatives of the DG Equation with respect to the random variables involved; it is a [3*15] matrix 
In a more detailed representation and forward omitting the time dependent $(\mathrm{t})$ symbol just for clarity, the LiDAR-based DG Equation can be expressed as shown in Equation 4.5.

$$
\left[\begin{array}{c}
X_{p} \\
Y_{p} \\
Z_{p}
\end{array}\right]_{l l}=\left[\begin{array}{c}
X_{b} \\
Y_{b} \\
Z_{b}
\end{array}\right]_{l l}+R_{\theta_{x}} R_{\theta_{y}} R_{\theta_{z}} *\left(R_{\tau_{x}} R_{\tau_{y}} R_{\tau_{z}} *\left[\begin{array}{c}
\rho \cos (\omega) \sin (\alpha) \\
\rho \cos (\omega) \cos (\alpha) \\
\rho \sin (\omega)
\end{array}\right]+\left[\begin{array}{c}
l x_{s}^{b} \\
l y_{s}^{b} \\
l z_{s}^{b}
\end{array}\right]\right)
$$

Where

$X_{p}, Y_{p}, Z_{p}$ are the $3 \mathrm{D}$ coordinates of the point in the local level frame

$X_{b}, Y_{b}, Z_{b}$ are the body frame origin time dependant 3D coordinates in the local level frame

$R_{\theta_{z}}$ is the time dependant rotation matrix from the body frame to the local level frame around the $\mathrm{z}$ axis with a yaw angle $\theta_{z}$

$R_{\theta_{y}}$ is the time dependant rotation matrix from the body frame to the local level frame around the y axis with a pitch angle $\theta_{y}$

$R_{\theta_{x}}$ is the time dependant rotation matrix from the body frame to the local level frame around the $\mathrm{x}$ axis with a roll angle $\theta_{x}$

$R_{\tau_{z}}$ is the rotation matrix from the sensor to the body frame around the $\mathrm{z}$ axis with a boresight angle $\tau_{z}$

$R_{\tau_{y}}$ is the rotation matrix from the sensor to the body frame around the y axis with a boresight angle $\tau_{y}$

$R_{\tau_{x}}$ is the rotation matrix from the sensor to the body frame around the $\mathrm{x}$ axis with a boresight angle $\tau_{x}$

$\omega$ is the laser beam vertical angle

$\rho$ is the range to the target

$\alpha$ is the laser horizontal angle

$l x_{s}^{b}, l y_{s}^{b}, l z_{s}^{b}$ are lever arm components between the sensor and the body frame origin

It is worth noting that the rotation sequence applied is a rotation around z-axis, followed by a rotation around the once-rotated $y$-axis, and lastly a rotation around the twice-rotated $x$-axis. 
Revisiting Equation 4.4 to calculate the uncertainty attached with the developed mapping system.

$$
C_{\text {sys }}=J C_{\text {par }} J^{T}
$$

The 15 random variables involved in Equation 4.4 can be grouped as shown in table 4.2

Table 4.2: The LiDAR DG Equation 15 random variables

\begin{tabular}{|l|c|}
\hline Group & Variables \\
\hline POS position & $X_{b}, Y_{b}, Z_{b}$ \\
\hline POS attitudes(roll, pitch, yaw) & $\theta_{x}, \theta_{y}, \theta_{z}$ \\
\hline $\begin{array}{l}\text { LiDAR(range, vertical angle, horizontal scan } \\
\text { angle) }\end{array}$ & $\rho, \omega, \alpha$ \\
\hline $\begin{array}{l}\text { Calibration-based parameters: } \\
\text { (Boresight angles) } \\
\left.\text { (lever arm sensor and body frame } l_{s}^{b}\right)\end{array}$ & $\tau_{x}, \tau_{y}, \tau_{z}$ \\
& $l x_{s}^{b}, l y_{s}^{b}, l_{s}^{b}$ \\
\hline
\end{tabular}

The Jacobian matrix $J$ is computed through the partial derivatives of the DG Equation with respect to the 15 random variables involved. The $J$ matrix is a [3*15] matrix. The $J$ matrix would be given by Equation 4.6. 
$J=\left[\begin{array}{lllllllllllllll}\frac{\partial X_{p}}{\partial X_{b}} & \frac{\partial X_{p}}{\partial Y_{b}} & \frac{\partial X_{p}}{\partial Z_{b}} & \frac{\partial X_{p}}{\partial \theta_{x}} & \frac{\partial X_{p}}{\partial \theta_{y}} & \frac{\partial X_{p}}{\partial \theta_{z}} & \frac{\partial X_{p}}{\partial \rho} & \frac{\partial X_{p}}{\partial \omega} & \frac{\partial X_{p}}{\partial \alpha} & \frac{\partial X_{p}}{\partial \tau_{x}} & \frac{\partial X_{p}}{\partial \tau_{y}} & \frac{\partial X_{p}}{\partial \tau_{z}} & \frac{\partial X_{p}}{\partial x_{s}^{b}} & \frac{\partial X_{p}}{\partial l y_{s}^{b}} & \frac{\partial X_{p}}{\partial l z_{s}^{b}} \\ \frac{\partial Y_{p}}{\partial X_{b}} & \frac{\partial Y_{p}}{\partial Y_{b}} & \frac{\partial Y_{p}}{\partial Z_{b}} \frac{\partial Y_{p}}{\partial \theta_{x}} & \frac{\partial Y_{p}}{\partial \theta_{y}} & \frac{\partial Y_{p}}{\partial \theta_{z}} & \frac{\partial Y_{p}}{\partial \rho} & \frac{\partial Y_{p}}{\partial \omega} & \frac{\partial Y_{p}}{\partial \alpha} & \frac{\partial Y_{p}}{\partial \tau_{x}} & \frac{\partial Y_{p}}{\partial \tau_{x}} & \frac{\partial Y_{p}}{\partial \tau_{z}} & \frac{\partial Y_{p}}{\partial l x_{s}^{b}} & \frac{\partial Y_{p}}{\partial l y_{s}^{b}} & \frac{\partial Y_{p}}{\partial l_{s}^{b}} \\ \frac{\partial Z_{p}}{\partial X_{b}} & \frac{\partial Z_{p}}{\partial Y_{b}} & \frac{\partial Z_{p}}{\partial Z_{b}} \frac{\partial Z_{p}}{\partial \theta_{x}} & \frac{\partial Z_{p}}{\partial \theta_{y}} & \frac{\partial Z_{p}}{\partial \theta_{z}} & \frac{\partial Z_{p}}{\partial \rho} & \frac{\partial Z_{p}}{\partial \omega} & \frac{\partial Z_{p}}{\partial \alpha} & \frac{\partial Z_{p}}{\partial \tau_{x}} & \frac{\partial Z_{p}}{\partial \tau_{x}} & \frac{\partial Z_{p}}{\partial \tau_{z}} & \frac{\partial Z_{p}}{\partial l x_{s}^{b}} & \frac{\partial Z_{p}}{\partial l y_{s}^{b}} & \frac{\partial z_{p}}{\partial l z_{s}^{b}}\end{array}\right]$

Differentiating $X_{p} Y_{p} Z_{p}$ w.r.t the 15 random values, and evaluating at the initial values for the 15 random variables as listed in Table 4.3 allows populating the Jacobian matrix.

Table 4.3: The 15 random variables used in the error propagation and their initial values

\begin{tabular}{|c|c|c|}
\hline Group & Variables & Initial values \\
\hline POS position & $X_{b}, Y_{b}, Z_{b}$ & $0,0,0$ \\
\hline POS attitudes(roll, pitch, yaw) & $\theta_{x}, \theta_{y}, \theta_{z}$ & $0^{\circ}, 0^{\circ}, 0^{\circ}$ \\
\hline $\begin{array}{l}\text { LiDAR(range, vertical angle, horizontal } \\
\text { scan angle) }\end{array}$ & $\rho, \omega, \alpha$ & $100,0^{\circ}, 0$ \\
\hline Calibration-based parameters: & & \\
\hline (Boresight angles) & $\tau_{x}, \tau_{y}, \tau_{z}$ & $0^{\circ}, 0^{\circ}, 0^{\circ}$ \\
\hline (lever arm sensor and body frame $l_{s}^{b}$ ) & $l x_{s}^{b}, l y_{s}^{b}, l z_{s}^{b}$ & $0,0,0$ \\
\hline
\end{tabular}

The variance-covariance matrix $C_{\text {par }}$ can be populated with the 15 random variables variances, which are typically reported as $(1 \sigma)$. Assuming that the variables are independent, the off-diagonal elements for the $C_{\text {par }}$ matrix would be zero. The developed system uncertainty predictive model can be used to estimate the uncertainty of any DG LiDAR-based mapping system.

The newly developed mapping system at hand uses the APX-15 as its POS and the VLP-16 as its RSS. The APX-15 reported typical precision performance is shown in Table 4.4. The manufacturer reports the precision values as RMS. Assuming unbiased normally distributed data, it is considered that the reported precision is the standard deviation $\sigma$. 
Table 4.4: APX-15 precision in different processing levels

\begin{tabular}{|l|l|l|l|l|}
\hline & SPS & RTK & PP_RTX & Post_Processed \\
\hline Position (m) & $1.5-3.0$ & $0.02-0.05$ & $0.03-0.06$ & $0.02-0.05$ \\
\hline Roll \& Pitch (deg) & 0.04 & 0.03 & 0.025 & 0.025 \\
\hline True Heading (deg) & 0.30 & 0.18 & 0.08 & 0.080 \\
\hline
\end{tabular}

As shown in Table 4.4, the position precision when using only the Standard Positioning Service (SPS) ranges from 1.5-3 m, while in the RTK using a base station and radio link, the position accuracy ranges from $0.02-0.05 \mathrm{~m}$. In addition, the PP_RTX uses a post-processed CenterPoint ${ }^{\circledR}$ RTX service from Applanix, yielding a typical positional accuracy in the range of 0.03-0.06 m. The highest positional accuracy can be achieved with post-processing ranging $0.02-0.05 \mathrm{~m}$, using a short baseline operation. The roll and pitch precision is $0.025^{\circ}$ for the PP_RTX and the Post_Processed, and 0.04 and 0.03 in the SPS and RTK, respectively. The true heading precision can reach 0.08 in the PP_RTX and Post_Processed, but remains 0.3 and 0.18 in the SPS and RTK, respectively. The least precise reported value in the SPS mode will be used for the least favorable uncertainty in the points' georeferenced 3D coordinates.

According to the reported precision in the different processing levels for the APX-15, the preferable case would achieve $0.02 \mathrm{~m}$ precision in the position and $0.025^{\circ}$ in roll and pitch, while achieving 0.08 precision in the heading with a post-processed solution. On the other hand, the least precise would be using it in SPS mode and would achieve $3.0 \mathrm{~m}$ precision in the position, $0.04^{\circ}$ in roll and pitch, and $0.3^{\circ}$ in the heading. To study the effect of the different achievable precisions on the uncertainty of the georeferenced 3D coordinates of the point cloud, several simulation runs have been carried out using different values of precision for the 15 parameters applied into the developed uncertainty predictive model. Starting with the most precise values reported for the APX-15, the precision value for each parameter was degraded and the effect on the output 3D point coordinates calculated. The results are presented in the next section.

Starting with the best performance, of the APX-15 in a post processed solution with values shown in Table 4.4 for the roll, pitch, yaw and position. For the LiDAR sensor, the VLP-16 range precision is reported as $3 \mathrm{~cm}$, assuming a calibrated sensor with very precise elevation angle $\omega$. The VLP-16 horizontal angle precision is not reported and so it is assumed to be half the angular resolution. For the calibration parameters, calibrated boresight angles precision of $0.03^{\circ}$ and lever arm precision of $0.02 \mathrm{~m}$ are assumed.

Subsequently, the uncertainty of the system with the least performance of the APX-15 in a SPS solution using the values shown in Table 4.4 for the roll, pitch, yaw and position can be calculated. The same precision for the VLP-16 as used in the favorable scenario. For the calibration parameters it is assumed a less precise calibrated boresight angles of $0.06^{\circ}$ and lever arms precision of 0.04 $\mathrm{m}$. Both the system components' precision values for the favorable (Post processed) and least favorable (SPS) scenario are shown in Table 4.5. 
Table 4.5: The System favorable precision (post-processed) \& least favorable SPS

\begin{tabular}{|c|c|c|}
\hline & $\begin{array}{l}\text { Post-processed } \\
\text { achievable } \\
\text { precision }\end{array}$ & $\begin{array}{l}\text { SPS achievable } \\
\text { precision }\end{array}$ \\
\hline$X_{b}$ & $0.02 \mathrm{~m}$ & $3 \mathrm{~m}$ \\
\hline$Y_{b}$ & $0.02 \mathrm{~m}$ & $3 \mathrm{~m}$ \\
\hline$Z_{b}$ & $0.02 \mathrm{~m}$ & $3 \mathrm{~m}$ \\
\hline$\theta \mathrm{x}$ & $0.025^{\circ}$ & $0.04^{\circ}$ \\
\hline$\theta \mathrm{y}$ & $0.025^{\circ}$ & $0.04^{\circ}$ \\
\hline$\theta z$ & $0.08^{\circ}$ & $0.3^{\circ}$ \\
\hline$\rho$ & $0.03 \mathrm{~m}$ & $0.03 \mathrm{~m}$ \\
\hline$\omega$ & $0^{\circ}$ & $0^{\circ}$ \\
\hline$\alpha$ & $0.05^{\circ}$ & 0.05 \\
\hline$\tau \mathrm{X}$ & $0.03^{\circ}$ & $0.06^{\circ}$ \\
\hline$\tau y$ & $0.03^{\circ}$ & $0.06^{\circ}$ \\
\hline$\tau \mathrm{z}$ & $0.03^{\circ}$ & $0.06^{\circ}$ \\
\hline$l x_{s}^{b}$ & $0.02 \mathrm{~m}$ & $0.04 \mathrm{~m}$ \\
\hline$l y_{s}^{b}$ & $0.02 \mathrm{~m}$ & $0.04 \mathrm{~m}$ \\
\hline$l z_{s}^{b}$ & $0.02 \mathrm{~m}$ & $0.04 \mathrm{~m}$ \\
\hline
\end{tabular}

The corresponding system performance metrics for three different ranges, namely, $25 \mathrm{~m}, 50 \mathrm{~m}$, and $100 \mathrm{~m}$ are shown in Table 4.6 below for the favorable and least favorable scenarios. The diagonal elements represent the uncertainty in the $X_{p} Y_{p} Z_{p}$ coordinates respectively, while the off-diagonal elements represent the co-variance values. 
Table 4.6: The system performance metrics (m), @ ranges 25m, 50m, 100m (favorable / least favorable scenario; diagonal elements uncertainty in the $X_{p} Y_{p} Z_{p}$ georeferenced coordinates respectively)

\begin{tabular}{|c|c|c|c|c|c|}
\hline \multicolumn{3}{|c|}{ Favorable scenario } & \multicolumn{3}{|c|}{ Least favorable scenario } \\
\hline \multicolumn{3}{|c|}{$25 m$} & \multicolumn{3}{|c|}{$25 m$} \\
\hline 0.05 & 0.01 & 0.01 & 3.00 & 0.03 & 0.03 \\
\hline 0.01 & 0.04 & 0.01 & 0.03 & 3.00 & 0.03 \\
\hline 0.01 & 0.01 & 0.03 & 0.03 & 0.03 & 3.00 \\
\hline \multicolumn{3}{|c|}{$50 m$} & \multicolumn{3}{|c|}{$\rho=50 m$} \\
\hline 0.09 & 0.03 & 0.03 & 3.01 & 0.05 & 0.05 \\
\hline 0.03 & 0.05 & 0.03 & 0.05 & 3.00 & 0.05 \\
\hline 0.03 & 0.03 & 0.04 & 0.05 & 0.05 & 3.00 \\
\hline \multicolumn{3}{|c|}{$100 m$} & \multicolumn{3}{|c|}{$\rho=100 m$} \\
\hline 0.18 & 0.05 & 0.05 & 3.05 & 0.10 & 0.10 \\
\hline 0.05 & 0.07 & 0.05 & 0.10 & 3.00 & 0.10 \\
\hline 0.05 & 0.05 & 0.07 & 0.10 & 0.10 & 3.00 \\
\hline
\end{tabular}

As shown in Table 4.6, for the favorable scenario, the system output vertical uncertainty at $25 \mathrm{~m}$ is $3 \mathrm{~cm}$ and the total horizontal uncertainty is $6 \mathrm{~cm}$, while the corresponding vertical uncertainty at the $100 \mathrm{~m}$ range is $7 \mathrm{~cm}$ and the total horizontal uncertainty is $19 \mathrm{~cm}$. These results and the horizontal-to-vertical uncertainty of a ratio of around two agrees very well with the results in [97], [98] and [99]. Moreover, the results conform to what is known of LiDAR's vertical accuracy being better than the horizontal one [97]. In the case of the least favorable SPS scenario, the position errors dominate the errors in the resulting point cloud, independently from the range which conforms to the results reported in [100].

It is worth noting that the beam divergence uncertainty will add to the resulting uncertainty of the point cloud, as the position will be recorded along the beam centerline, whereas the actual point location may be anywhere within the beam footprint. The beam divergence uncertainty effect is directly proportional to the range to the targets. The VLP-16 beam horizontal divergence is $3 \mathrm{mrad}$ and the vertical beam divergence is $1.5 \mathrm{mrad}$. By integrating the beam divergence to the uncertainty of the resultant point cloud and assuming that the peak power occurs near the beam centerline, one-quarter of the beam diameter would be a conservative estimate for the beam divergence uncertainty [101]. Thus, integrating the VLP-16 beam divergence to the uncertainty of the resultant point cloud yields the total uncertainty for ranges of $25 \mathrm{~m}, 50 \mathrm{~m}$, and $100 \mathrm{~m}$, as shown in Table 4.7 for the favorable/least favorable scenarios 
Table 4.7: The system performance metrics (m), @ ranges 25m, 50m, 100m (favorable/ least favorable scenario; uncertainty in the $X_{p} Y_{p} Z_{p}$ georeferenced coordinates including beam divergence uncertainty)

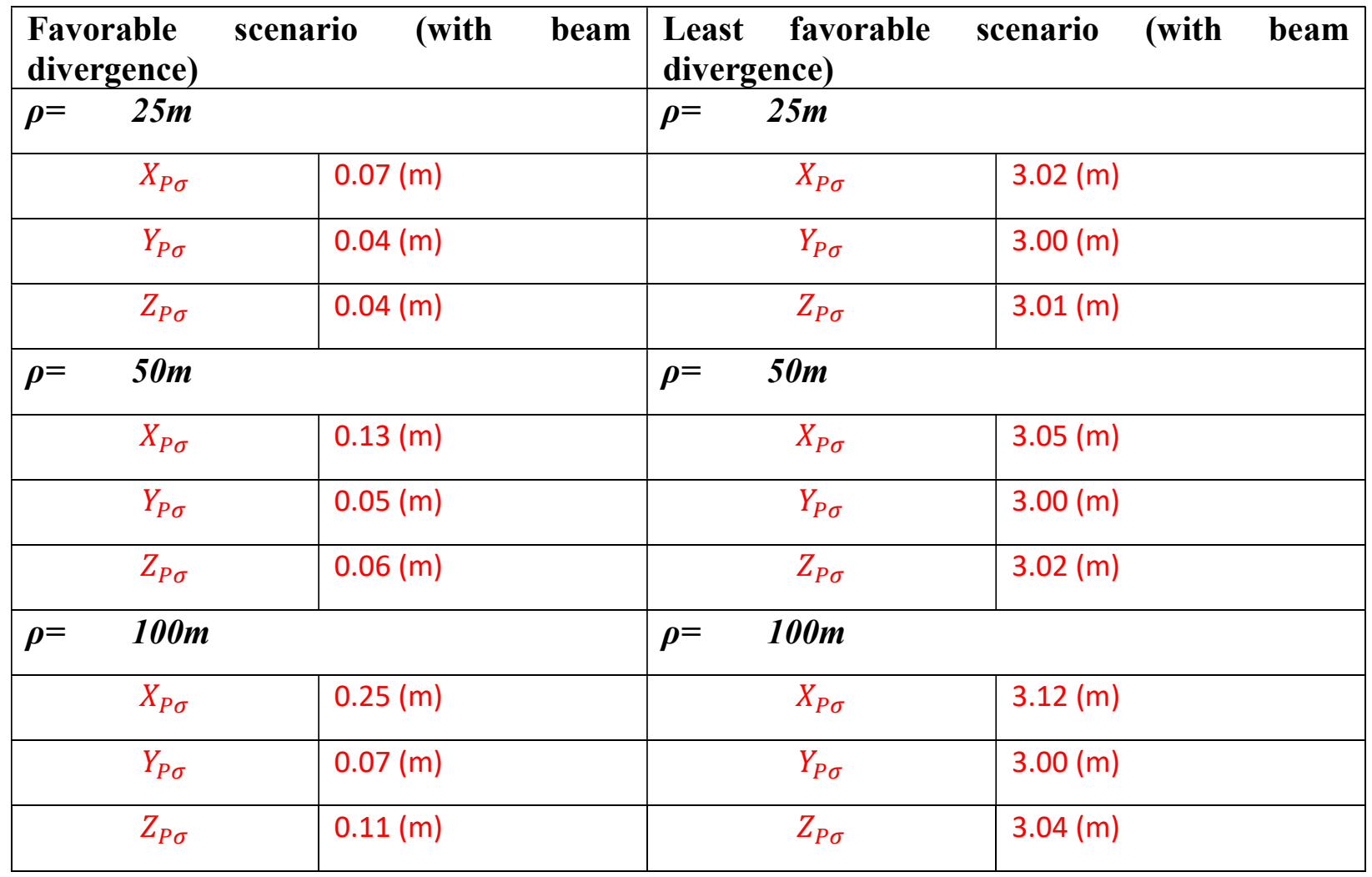

Again, as shown in Table 4.8, the most dominant error source affecting the resultant point cloud is poor GNSS positioning accuracy. In this case, the beam divergence effect is almost negligible even with the 100 range, which is the max achievable range for the VLP-16. The rigorous uncertainty predictive model developed in this study helps to provide a tool for the resultant point cloud accuracy estimate, which is especially useful in instances where it is difficult to find a more accurate data source for the accuracy-checking process

Applying the developed uncertainty predictive model with the favorable scenario and including the beam divergence, the total horizontal uncertainty is $8 \mathrm{~cm}$ and the vertical uncertainty is $4 \mathrm{~cm}$ $(1 \sigma @ 25 \mathrm{~m})$. Thus, combining the specifications for the system components and its uncertainty the system specifications can be summarized as shown in Table 4.8. 
Table 4.8: The developed mapping system specifications

\begin{tabular}{|l|l|}
\hline Attribute & Value \\
\hline Scanner & VLP-16 (easily upgradable) \\
\hline POS & APX-15 V3 \\
\hline Wavelength & $903 \mathrm{~nm}$ \\
\hline System uncertainty $(1 \sigma @ 25 \mathrm{~m})$ & Horizontal: $8 \mathrm{~cm}$, Vertical: $4 \mathrm{~cm}$ \\
\hline Dimensions & Dia $10.3 \mathrm{~cm} /$ height $15 \mathrm{~cm}$ \\
\hline HFOV & $360^{\circ}$ \\
\hline VFOV & $\begin{array}{l}+15^{\circ} \text { to }-15^{\circ} \text { (up to } 360^{\circ} \text { with the new mapping } \\
\text { scheme) }\end{array}$ \\
\hline Data Pts/Sec & 300,000 (single return); 600,000 (dual return) \\
\hline Power consumption & $19 \mathrm{~W}$ (Autonomy $\sim 1.2 \mathrm{hr})$ \\
\hline Operating Temperature & $-10^{\circ} \mathrm{C}$ to $+60^{\circ} \mathrm{C}$ \\
\hline Mapping Modes & $\mathrm{ALL}$; stationary, mobile, ULS \\
\hline
\end{tabular}

It is worth highlighting that for the data processing of the system realization in the next chapters the processing of the GNSS/INS data has been carried out in the POSPAC environment.

Four sets of measurements has been determined and programed in POSPAC:

1- The mounting angles from the Reference frame (the VLP-16 in the research at hand) to the IMU body frame (APX-15), following the Tate-Bryant sequence of rotations (i.e rotation around $\mathrm{z}$, then rotation around $\mathrm{y}$, then rotation around $\mathrm{x}$ )

2- The mounting angles from the Vehicle frame to the reference frame following the TateBryant sequence of rotations (i.e rotation around $\mathrm{z}$, then rotation around $\mathrm{y}$, then rotation around $\mathrm{x}$ )

3- The lever arm between the Reference frame and the IMU body frame resolved in the Reference frame

4- The lever arm between the Reference frame and the antenna phase center APC resolved in the vehicle frame

POSPAC then uses these values in the trajectory calculation, then reports the position and orientation considering the LiDAR sensor as the reference frame (i.e directly relating the position and orientation of the LiDAR sensor to the ground), thus the applied equation for the data geo referencing is as follows: 


$$
P_{m}=r_{m}(t)+R_{s}^{m}(t) \cdot P_{s}(t)(4.7)
$$

Where

$P_{m}$ is the $3 \mathrm{D}$ coordinates of the point in the mapping frame

$r_{m}(t)$ is the time dependant $3 \mathrm{D}$ coordinates of the sensor frame origin in the mapping frame $R_{S}^{m}(t)$ is the time dependant rotation matrix from the sensor frame to the mapping frame $P_{S}$ is the $3 \mathrm{D}$ point coordinates in the sensor frame 


\section{MAPPING SYSTEM REALIZATION: STATIONARY, MOBILE MODES OF OPERATION, AND POINT CLOUD COLORIZATION}

Several different data collection missions utilizing the developed mapping system have been carried out in diverse environments using the three modes of operation discussed in section 4.3 (stationary, mobile and airborne). The uniqueness of the developed system is the ability to readily use it in different modes of operation, independent of the platform used and following a plug-n-go concept. A detailed discussion of the data collection missions performed and the system performance metrics for the different modes of operation is presented in the following section. In addition, the usage of a very low-cost ( 250 USD) MoCAP sensor in a new mapping scheme is presented and evaluated.

\subsection{Data Collection in Stationary Mode (LiDAR)}

In order to test the system performance in stationary mode, a data collection mission has been carried out in the form of the mapping of a building façade. The building chosen is the EPH building façade located at the intersection of Gerrard and Church Street on the Ryerson University campus in downtown Toronto, Canada. As described in section 4.3.1, the VLP-16 sensor pitch (nose up-down around the sensor's X-axis) is incrementally changed to densify the resulting point cloud by filling the gaps caused by the sensor coarse vertical resolution. This also serves to increase the sensor's limited vertical field of view. According to the results from the uncertainty predictive model developed in this proposal, the short range to the target $(\sim 6 \mathrm{~m})$ will limit the uncertainty caused by the less precise measurements for the attitude angles, and thus a very low-cost IMU has been tested.

The IMU selected after a thorough search is the yostlabs 3-Space ${ }^{\mathrm{TM}}$ Data Logger sensor. It is a miniature Attitude and Heading Reference System (AHRS). The sensor specifications according to its specifications sheet is presented in Table 5.1. The AHRS sensor was attached on top of the VLP-16, with the aim of maintaining alignment between the VLP-16 and IMU centers. A customer-grade Garmin GPS 18x LVC sensor has been used for the position recording and for the time-stamping of the VLP-16. Both the IMU and the GPS sensors used are shown in Fig. 5.1. The GPS 18x LVC sensor output data is given in NMEA 0183 format and provides a pulse-per-second logic-level output with a rising edge aligned to within 1 microsecond of UTC to time-stamp the VLP-16 data. Because it accepts the WAAS and EGNOS corrections, the position accuracy is in the m-level. The interfacing of the GPS 18x LVC sensor and the VLP-16 has been carried out as described in section 3.3. The system was mounted on a camera tripod with its spinning axis almost vertical and the incremental pitch of the system $\beta$ angle has been applied. 

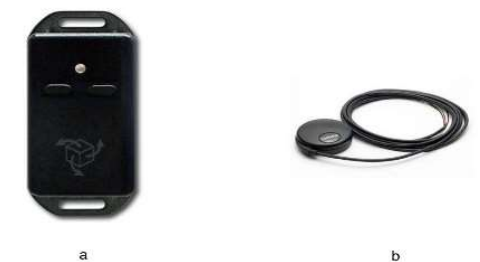

Fig 5.1: Very Low cost IMU and GPS ,

a) The Yostlabs Data logger AHRS and b) Garmin GPS18x LVC

Table 5.1: The Yostlabs Datalogger AHRS specifications

\begin{tabular}{|l|l|}
\hline \multicolumn{2}{|c|}{ Attributes } \\
\hline Dimensions & $35 \mathrm{~mm} \times 60 \mathrm{~mm} \times 15 \mathrm{~mm}$ \\
\hline Weight & 28 grams \\
\hline Supply voltage & $+5 \mathrm{v}$ USB \\
\hline Battery technology & rechargeable Lithium-Polymer \\
\hline Battery lifetime & $5+$ hours continuous use at full performance \\
\hline Storage interface & \\
\hline Communication interfaces & SD card \\
\hline Orientation range & USB 2.0 \\
\hline Orientation accuracy & $360^{\circ}$ about all axes \\
\hline Orientation resolution & $\pm 1^{\circ}$ for dynamic conditions \& all orientations \\
\hline Orientation repeatability & \\
\hline
\end{tabular}


The original VLP-16 readings, limited field of view, the incremental pitching applied and an optical imagery of the façade are shown in Fig 5.2.
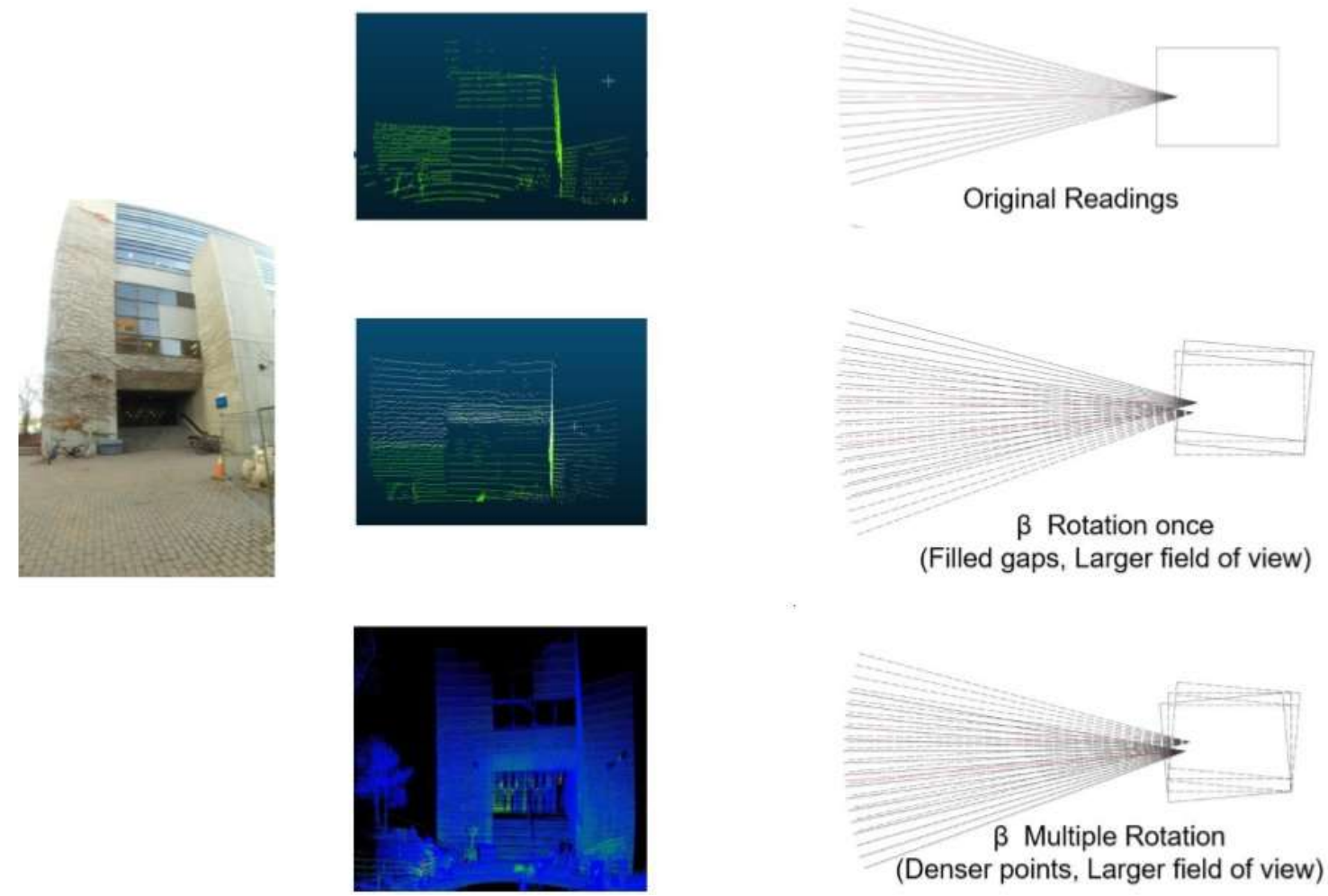

Fig 5.2: The system rotational motion $\beta$ in stationary mode

(Façade image (left), original sensor reading (top row), $\beta$ rotation once (middle row), Multiple $\beta$ rotations (bottom row))

As can be seen in Fig. 5.2, the original reading of the sensor (shown in the top row) suffers from the vertical gaps between the sensor scanlines and has a limited vertical field of view that does not allow the scan of the building façade. The rotation of the sensor around its X-axis with an angle $\beta$ once (as shown in the middle row) increases the sensor field of view and densifies the point cloud; it also decreases the vertical scanline gaps of the sensor readings. The incremental $\beta$ rotation of multiple times allows a much more densified point cloud of the building façade. As well, to a very large extent, it mitigates the vertical scanline gaps between the sensor scan lines, thus densifying the point cloud and allowing the mapping of the building façade (as shown in the bottom row).

The challenge to meet with the usage of the separate AHRS and GPS sensors is that the data must be first referenced to the same time in order for the different data streams from the AHRS, GPS and VLP-16 to be fused. The GPS sensor time-stamps the VLP-16 with UTC, but the AHRS has a very imprecise internal clock, and the imprecise synchronization will negatively impact the data fusion process. In response to this problem, the AHRS internal clock timing has been augmented by the corrections from the Network Time Protocol (NTP) strata 1 servers to obtain a UTC time 
with higher precision, which is then linked to the time-stamped VLP-16 data. The NTP was originally developed to synchronize the clocks of network infrastructure devices (e.g., servers, routers, switches, and computers) over a network. The NTP synchronizes the time of the network infrastructure devices to an external accurate time source (typically an atomic clock). Time accuracies ranging from 1 to $50 \mathrm{~ms}$ are possible with NTP, based on different network conditions. The DG equation is then applied to obtain a georeferenced point cloud for the building façade. A sample of the raw readings of the VLP-16 point cloud is shown in Table 5.2 and the corresponding façade scan is shown in Fig. 5.3.

Table 5.2: VLP-16 point cloud sample raw data

\begin{tabular}{|c|c|c|c|c|}
\hline Intensity & Laser_id & Horizontal angle & Distance $m$ & Adjusted time \\
\hline 7 & 0 & 15 & 5.112 & 73791131933 \\
\hline 9 & 1 & 16 & 10.544 & 73791131935 \\
\hline 9 & 2 & 17 & 5.822 & 73791131937 \\
\hline 2 & 3 & 17 & 9.83 & 73791131940 \\
\hline 7 & 4 & 18 & 6.722 & 73791131942 \\
\hline 2 & 5 & 19 & 15.796 & 73791131944 \\
\hline 3 & 6 & 20 & 7.954 & 73791131946 \\
\hline 2 & 7 & 21 & 12.588 & 73791131949 \\
\hline 21 & 8 & 22 & 8.646 & 73791131951 \\
\hline 3 & 9 & 23 & 12.664 & 73791131953 \\
\hline 15 & 10 & 23 & 8.896 & 73791131956 \\
\hline 3 & 11 & 24 & 12.688 & 73791131958 \\
\hline 10 & 12 & 25 & 9.424 & 73791131960 \\
\hline 9 & 14 & 27 & 9.962 & 73791131965 \\
\hline 5 & 15 & 27 & 11.784 & 73791131967 \\
\hline 6 & 0 & 35 & 5.098 & 73791131988 \\
\hline 4 & 1 & 36 & 9.48 & 73791131990 \\
\hline 9 & 2 & 37 & 5.82 & 73791131992 \\
\hline 3 & 3 & 37 & 12.462 & 73791131995 \\
\hline 9 & 4 & 38 & 6.736 & 73791131997 \\
\hline 5 & 5 & 39 & 12.464 & 73791131999 \\
\hline 6 & 6 & 40 & 7.95 & 73791132002 \\
\hline 5 & 7 & 41 & 12.54 & 73791132004 \\
\hline 19 & 8 & 42 & 8.656 & 73791132006 \\
\hline 3 & 9 & 43 & 12.596 & 73791132009 \\
\hline 15 & 10 & 43 & 8.886 & 73791132011 \\
\hline 3 & 11 & 44 & 12.688 & 73791132013 \\
\hline 12 & 12 & 45 & 9.442 & 73791132016 \\
\hline 34 & 14 & 47 & 9.482 & 73791132020 \\
\hline
\end{tabular}




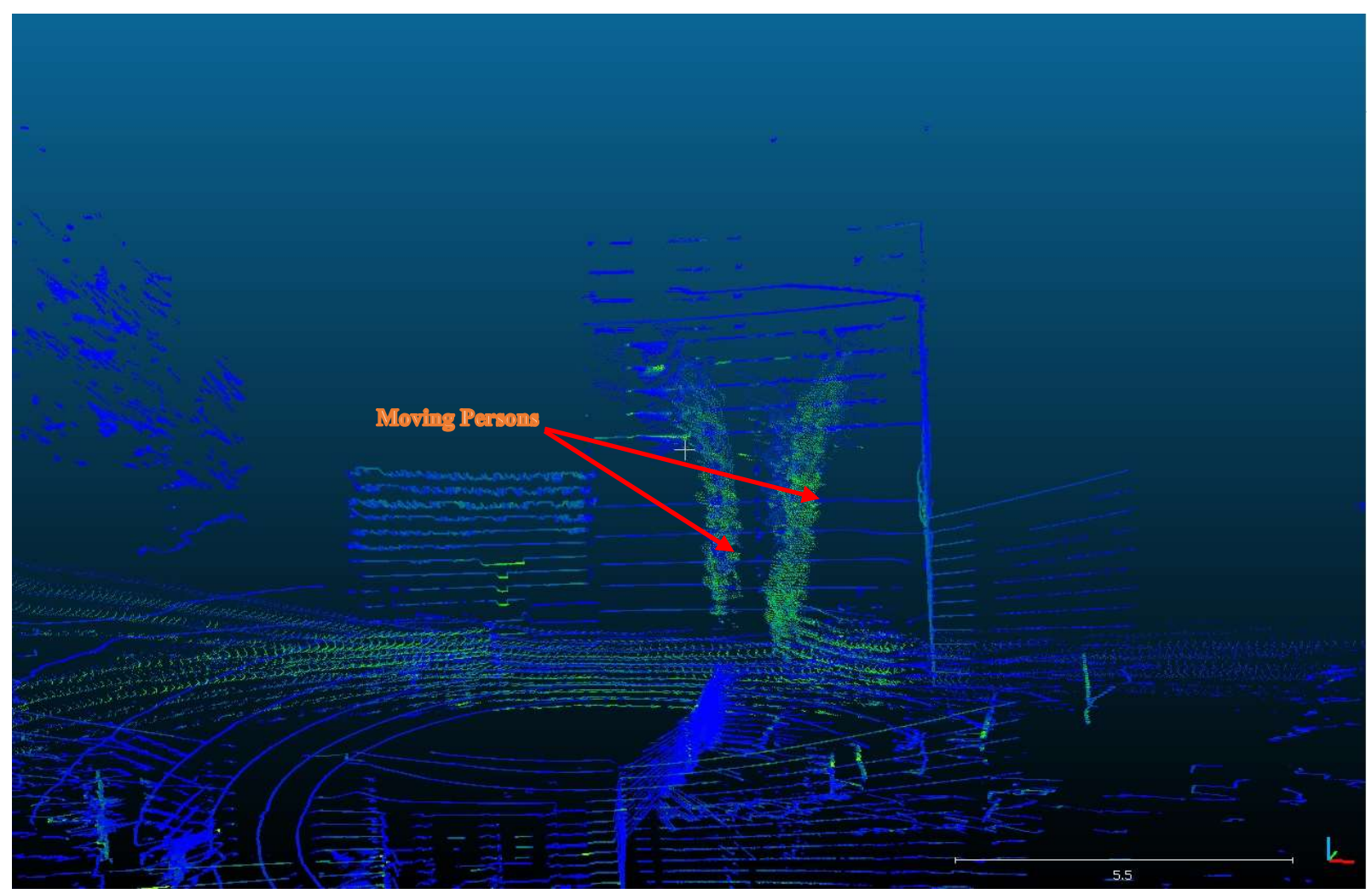

Fig 5.3: Original VLP-16 scan with vertical coarse resolution and limited FOV

As can be seen in Fig. 5.3, the VLP-16 scanning mechanism originally designed for autonomous navigation will not fit its utilization in stationary mode to map static objects, as its coarse vertical resolution and limited VFOV will hinder its usability in such an application. According to the new mapping scheme proposed in section 4.3.1, the sensor limitations in terms of the coarse vertical resolution and limited VFOV are greatly mitigated. Consequently, the laser data was parsed and the laser ID was used to retrieve the beam vertical angle $\omega$, the horizontal angle $\alpha$, and the range p. Equation 4.2 was then applied to calculate the points scanned referenced to the VLP-16 reference frame, and a virtual coordinate system for the AHRS was defined to align with the VLP16 axes directions. The measured attitudes from the AHRS, which is time-stamped with the augmented NTP corrections, were then used to record the sensor orientation for each firing. The NMEA received by the VLP-16 from the GPS18x LVC sensor was used to record the sensor position at each beam-firing. Interpolating the recorded AHRS attitudes and joining them with the VLP-16 beam-firing according to the linked time-stamp was then carried out. The DG equation is applied to georeference the resultant point cloud. The resultant point cloud and the corresponding optical imagery are depicted in Fig. 5.4. 


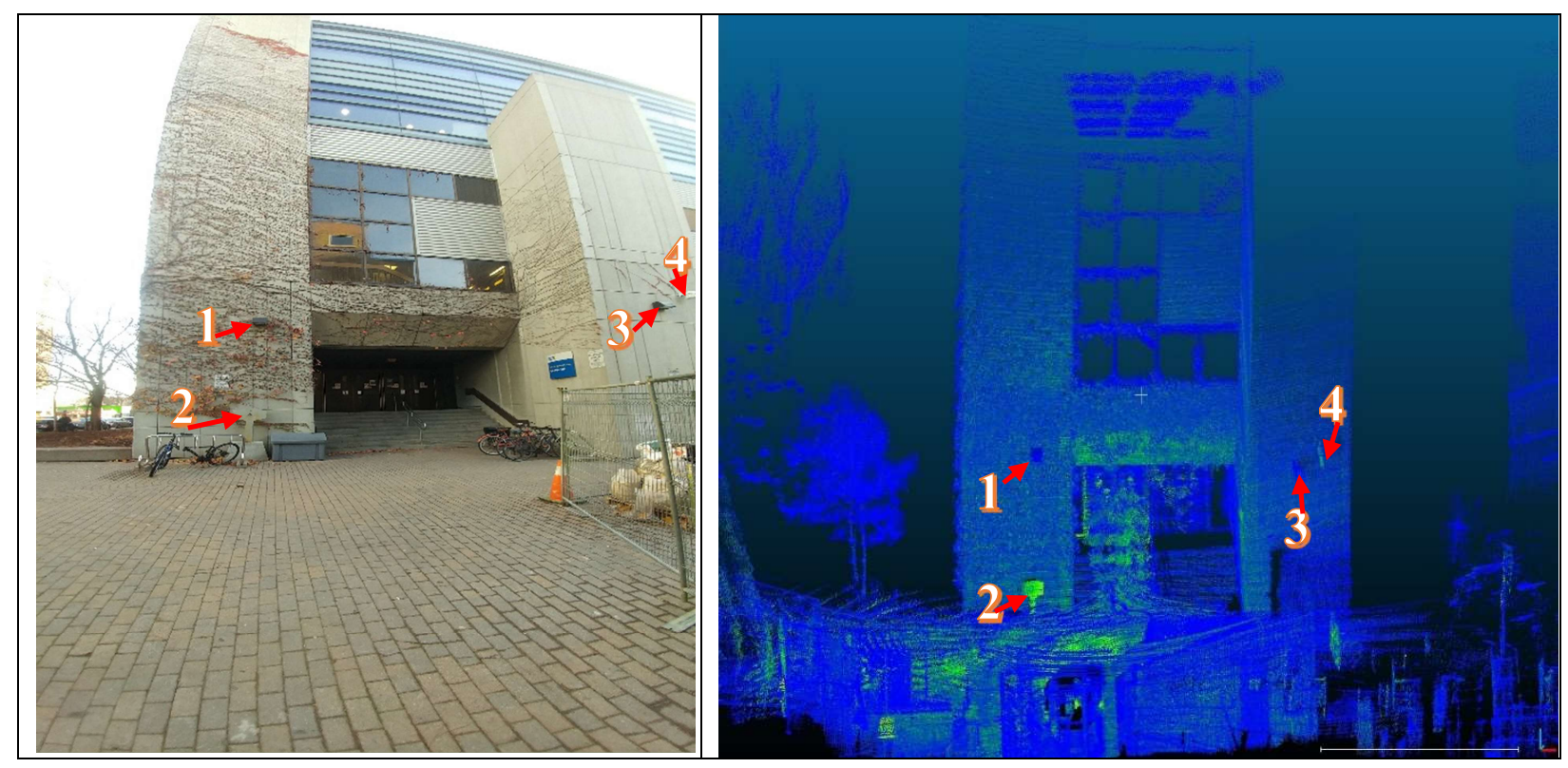

Fig 5.4: EPH building processed point cloud and the corresponding optical imagery

Both qualitative and quantitative assessments of the resultant point cloud have been implemented. First, the qualitative assessment of the new mapping scheme has been done by comparing Figs. 5.3 and 5.4. The comparison of the two figures clearly proves that the new mapping scheme mitigates the VLP-16 vertical coarse resolution and increases its vertical field of view. In addition, small objects are detectable, as highlighted by the numbered objects 1,2,3 and 4 in the optical imagery and the corresponding mapped objects in the georeferenced point cloud. Some fine details captured by the proposed new mapping scheme are highlighted in a side and front view in Figs. 5.5 and 5.6, respectively. The objects denoted by the number 1 in Fig. 5.5 are the returns measured from objects inside the building, as the laser beams penetrated the glass areas on the entrance doors.

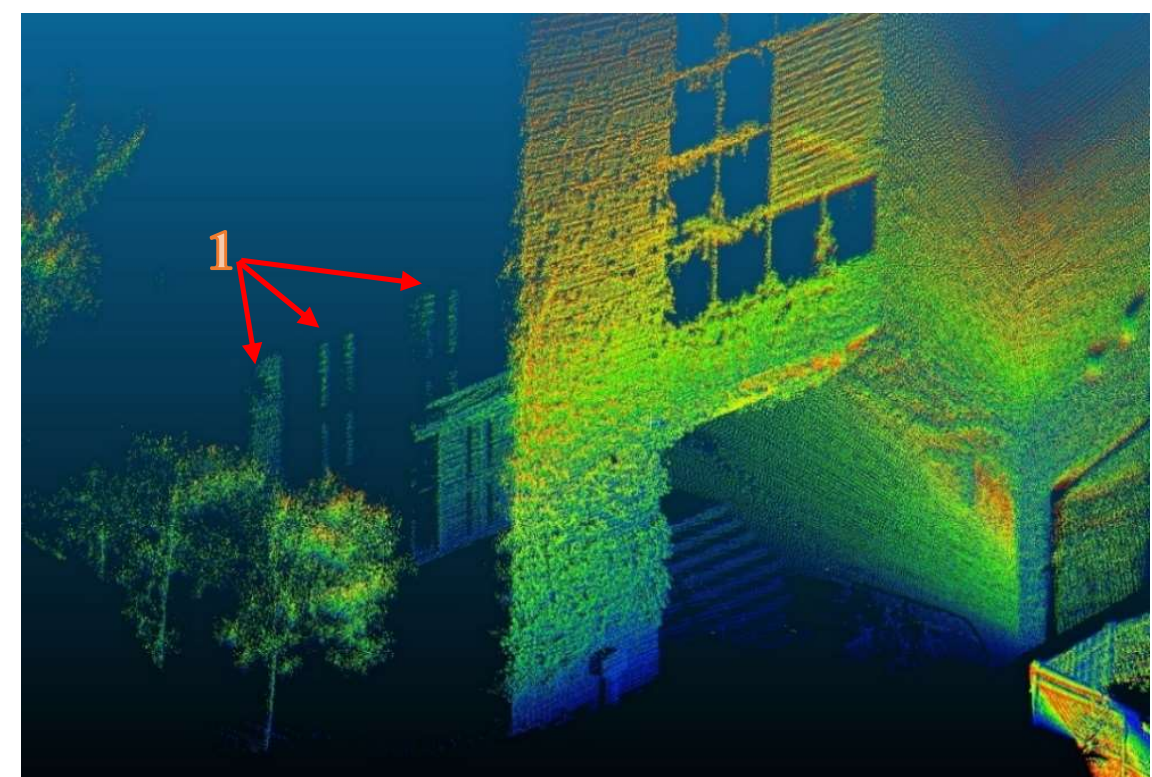

Fig 5.5: EPH side view fine details 


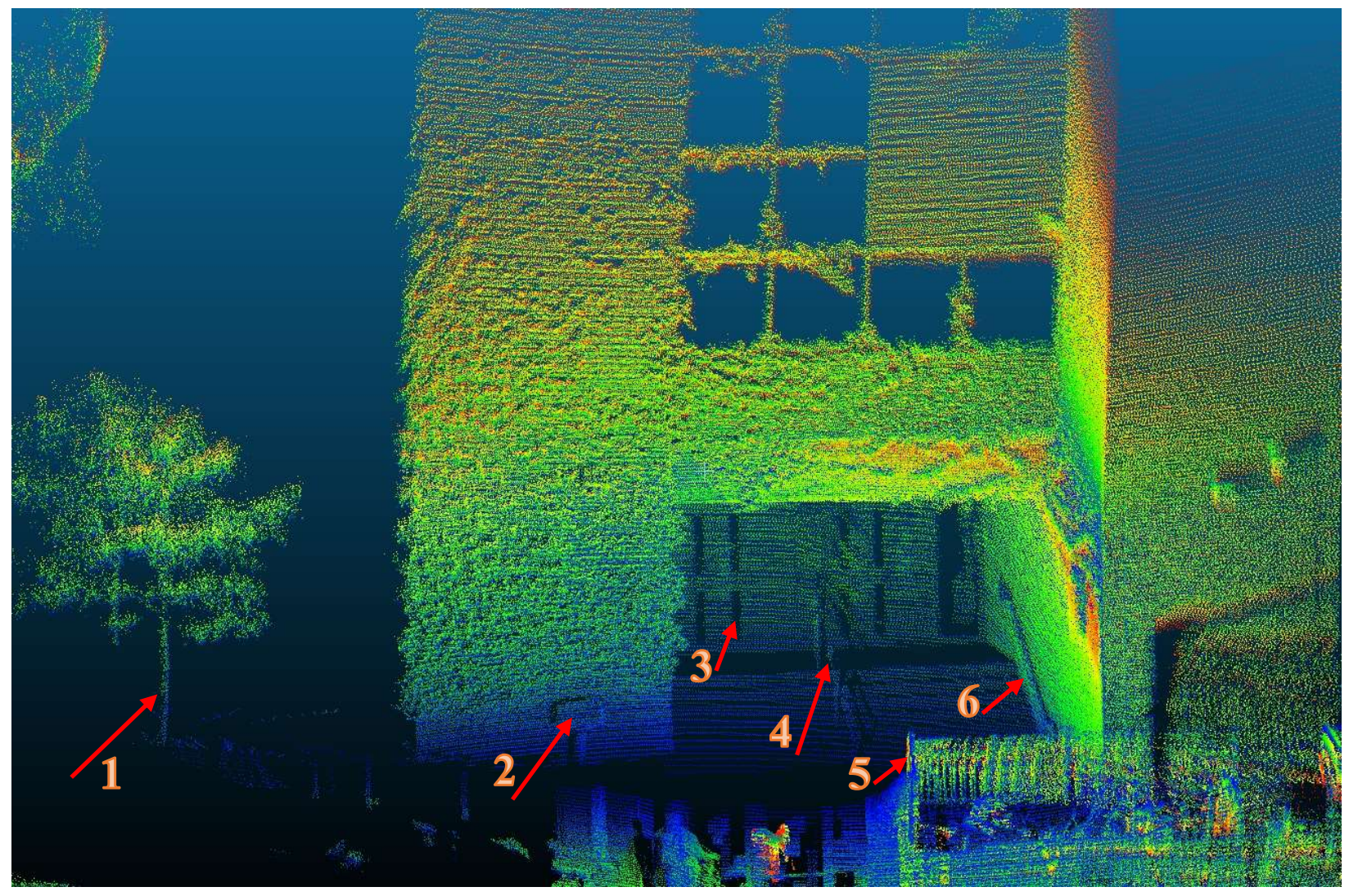

Fig 5.6: EPH front view fine details

Figure 5.6 shows a number of fine details that are captured by the new mapping scheme. A narrow tree stem (1), a sign (2), door openings (3), a fence (5), and even fine details such as the stair rails (denoted by numbers 4 and 6) have been captured by the new mapping scheme. An optical imagery for the EPH building entrance is depicted in Fig. 5.7.

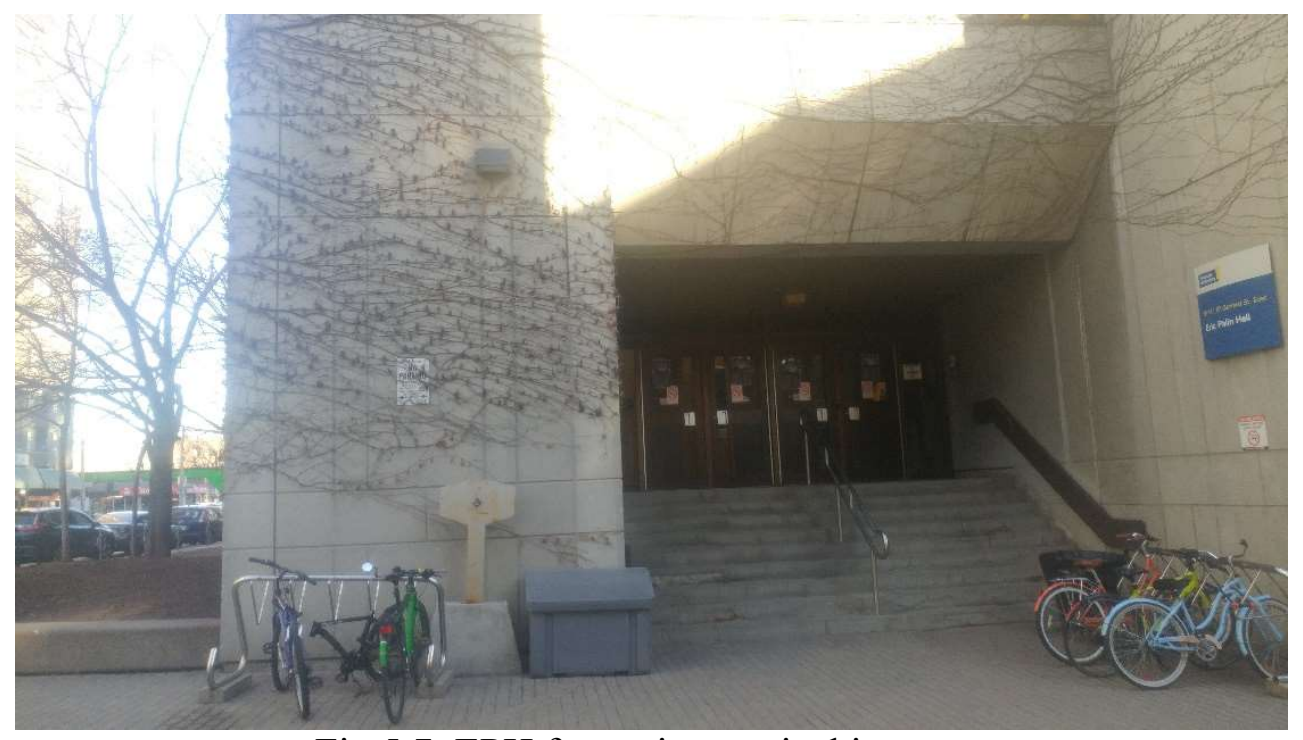

Fig 5.7: EPH front view optical imagery 
Moreover, a quantitative assessment has been carried out by comparing the resultant point cloud to a state-of-the-art terrestrial laser scanner point cloud. The Polaris scanner used for the quantitative assessment is a high-end terrestrial laser scanner that costs $~ 150,000$ USD and has a $5 \mathrm{~mm}$ accuracy. The Polaris scan is presented in Fig. 5.8.

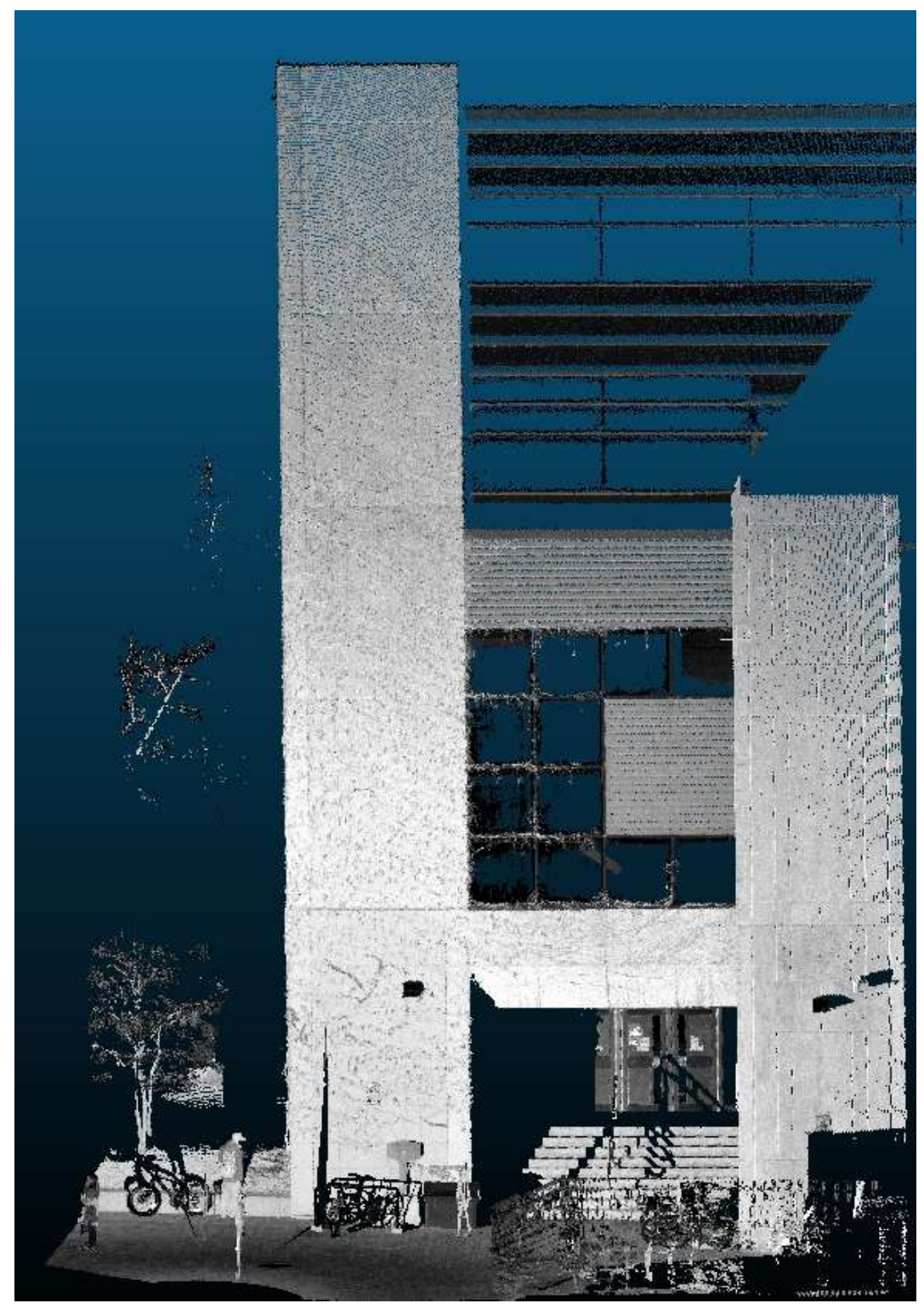

Fig 5.8: EPH building (Polaris scan)

Figure 5.8 shows the level of fine details expected from a high-end terrestrial laser scanner designed specifically to be used in stationary mode. However, its cost is considered prohibitive to many end-user segments. Moreover, its ease of deployment is questionable, given its relatively heavy weight and bulky size. The proposed alternative new mapping scheme costs less than onetenth of the price of the high-end laser and features ease of deployment as well as the ability to capture enough fine details to be deemed sufficient for many applications, such as 3D models of 
buildings, statues and objects; video fly-throughs of a scene; and inspection-related tasks. As some applications might be more interested in the geometry measurements of the façade abstraction, plane-fitting using RANdom SAmple Consensus (RANSAC) has been carried out for the point cloud, along with measured dimensions for both the proposed system resultant point cloud and the corresponding ground truth data. The comparison and deviation are presented in Table 5.3.

Table 5.3: Sample Measurements (point cloud-ground truth)

\begin{tabular}{|l|l|l|}
\hline Dimension & $\begin{array}{l}\text { Measured (Ground } \\
\text { Truth) }\end{array}$ & $\begin{array}{l}\text { Measured (3D point } \\
\text { Cloud) }\end{array}$ \\
\hline Basket Dia & $0.7 \mathrm{~m}$ & $0.6 \mathrm{~m}$ \\
\hline Sign Width & $0.7 \mathrm{~m}$ & $0.67 \mathrm{~m}$ \\
\hline Sign column & $0.16 \mathrm{~m}$ & $0.18 \mathrm{~m}$ \\
\hline $\begin{array}{l}\text { MON entrance } \\
\text { Width }\end{array}$ & $3.45 \mathrm{~m}$ & $3.4 \mathrm{~m}$ \\
\hline $\begin{array}{l}\text { MON entrance } \\
\text { Height }\end{array}$ & $2.44 \mathrm{~m}$ & $2.32 \mathrm{~m}$ \\
\hline EPH entrance width & $5.52 \mathrm{~m}$ & $5.56 \mathrm{~m}$ \\
\hline $\begin{array}{l}\text { Half EPH entrance } \\
\text { width }\end{array}$ & $2.86 \mathrm{~m}$ & $2.84 \mathrm{~m}$ \\
\hline $\begin{array}{l}\text { Height EPH } \\
\text { entrance }\end{array}$ & $2.63 \mathrm{~m}$ & $2.65 \mathrm{~m}$ \\
\hline Fence(p1) & $2.34 \mathrm{~m}$ & $2.43 \mathrm{~m}$ \\
\hline Fence(p2) & $2.36 \mathrm{~m}$ & $2.3 \mathrm{~m}$ \\
\hline Fence(p3) & $2.24 \mathrm{~m}$ & $2.3 \mathrm{~m}$ \\
\hline Wall Behind the sign & $3.63 \mathrm{~m}$ & $3.64 \mathrm{~m}$ \\
\hline
\end{tabular}


Calculating the RMSE for the sample measurements extracted from the resultant point cloud and the ground truth data yields a value of $0.06 \mathrm{~cm}$, which is a highly promising result, considering the very low cost of the IMU sensor used and the lack of boresight calibration. The rotation angles within the new mapping scheme application (pitching the sensor around its X-axis) is depicted in Fig. 5.9. 


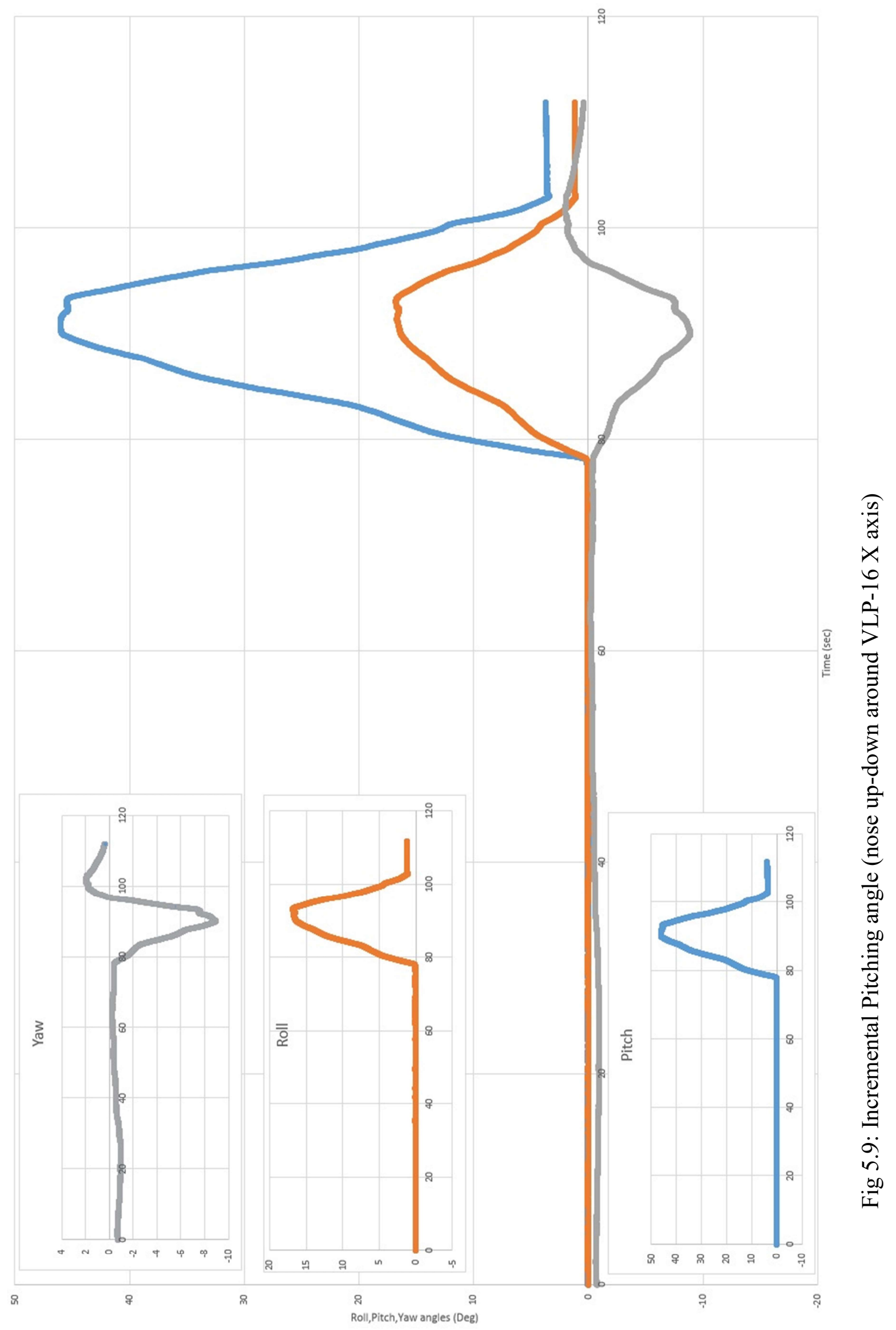


As can be seen, Fig. 5.9 shows the incremental change in the pitching angle. This change is intended to fill the gaps in the VLP-16 scanning due to the coarse vertical resolution; it is also intended to increase its total VFOV. As well, the figure shows a small change in the yaw and roll angle, caused by the interactive rotation applied to the system as a whole. The rising edge of the incremental change in the pitching angle has been used in the analysis in order to decrease the probability of duplicate points to be scanned, which proved sufficient in the façade mapping by increasing the VLP-16 total VFOV and densifying the resultant point cloud. The absolute geolocation position of the scanned façade will ultimately depend on the accuracy of the GNSS receiver used.

The APX-15 POS has also been tested for façade mapping. Although this device is originally optimized to be used in UAV dynamic application, its new utilization in stationary mode for façade mapping yielded a clear resultant point cloud, as depicted in Figs. 5.10. As can be seen, the geometry measurements from the utilization of the APX-15 with the system are almost identical to those obtained with the AHRS low-cost sensor, due to the proximity of the system to the mapped target. On the other hand, the geolocation precision using the APX-15 even without a base station reaches the decimeter level.

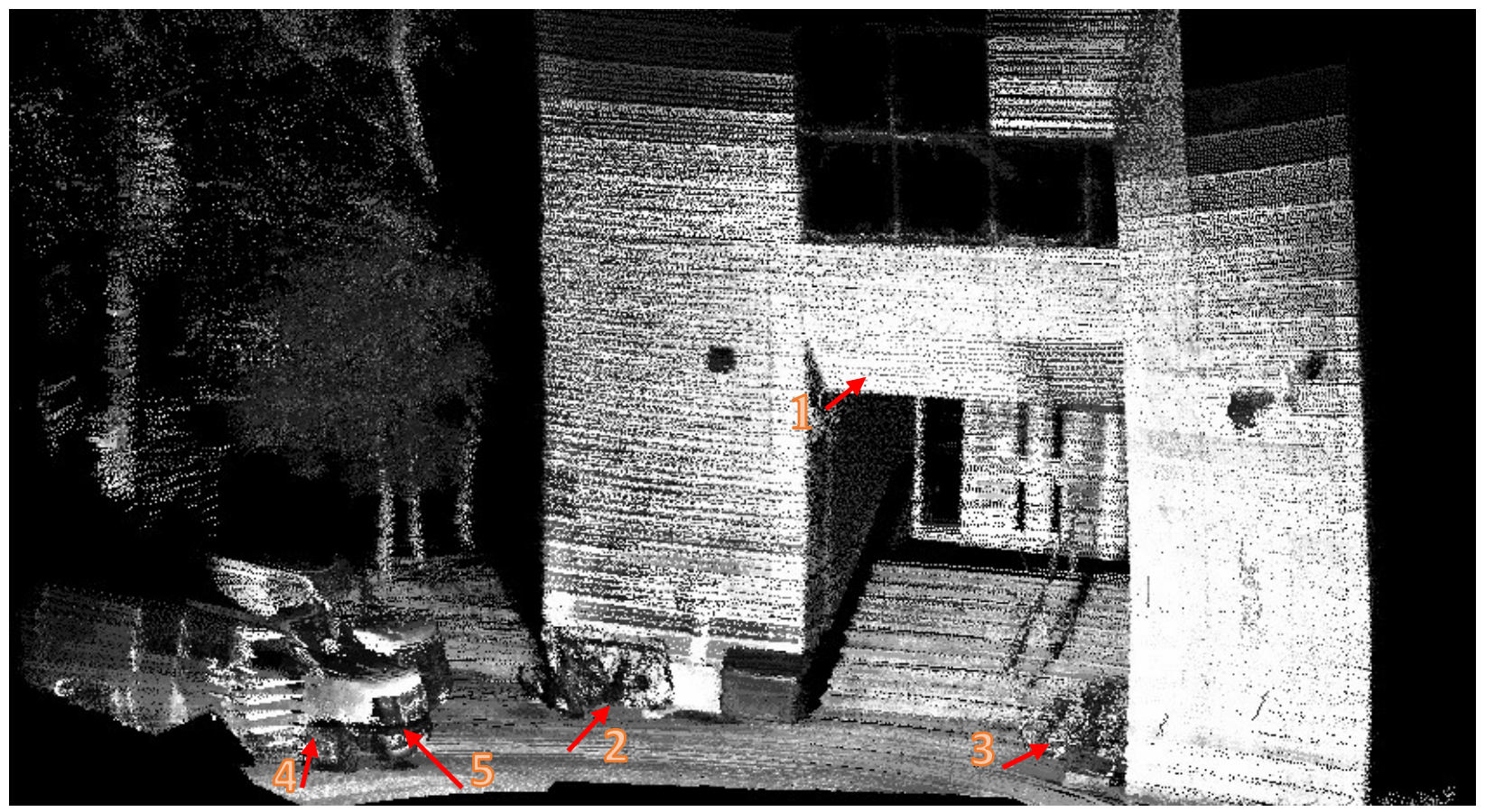

Fig 5.10: EPH façade (the system with APX-15) fine details

As it is shown in Fig 5.10 fine details are correctly mapped with the new system despite that the APX-15 is originally designed for UAV dynamic application and its utilization in a stationary application is a new type of its utilization. Object numbered 1 in Fig 5.10 correctly represent a sloped surface, object 2,3 shows bike racks and bikes, while object number 4 clearly present the van tire while object 5 represent the van's front lights. 
It is worth noting that the Polaris scanner has a $120^{\circ}$ vertical field of view, which is four times that of the VLP-16; furthermore, it has an angular resolution of $12 \mu \mathrm{rad}$, which is orders of magnitude better than that of the VLP-16. These enhanced features explain the precise verticality of the objects depicted by the Polaris. On the other hand, to be able to use the low-cost VLP-16 in such an application, a complicated process has to be implemented as presented and summarized in Chapter 3 and Fig 3.10.The process includes, to synchronise its readings with the attitudes and position readings from the multimodal sensors and to apply a kinematical change to the sensor pitch angle to be able to map the building façade. Additionally, a calibration mission is required to solve for the boresight and lever arm misalignments.

Overall, fine details have been correctly mapped with the proposed system, and reasonably precise measurements can be made with a RMSE of $0.06 \mathrm{~m}$ as calculated from the data presented in Table 5.3. The introduction of a very low-cost AHRS and customer-grade GPS sensor proved successful. Thus, the Polaris output only depends on the scanner characteristics, whereas to be able to map the building façade with the new mapping system using the VLP-16, its pitch angle needs to be incrementally changed with a POS. The new mapping scheme adds to the versatility of the developed mapping system architecture and introduces a low-cost alternative through the combination of the VLP-16, yostlabs AHRS, and the Garmin 18x LVC. The novel scheme allows the VLP-16 and the APX-15, which were both developed for kinematic environments, to be used in stationary mode. 


\subsection{Data Collection in Stationary Mode (Optical Imagery)}

The utilization of an SfM workflow to build a 3D point cloud from multiple overlapping imagery has been implemented for the EPH building façade. The façade has been captured with optical imagery from a cellphone camera (specifications shown in Table 5.4). The images have been captured from different positions and viewing angles to enhance the geometry needed for better 3D point geometry retrieval through bundle adjustment. The SfM model workflow was run in a Pix 4D environment. The produced 3D point cloud model is presented in Fig. 5.11.

Table 5.4: Cellphone camera specs used to capture EPH façade imagery

\begin{tabular}{|l|l|}
\hline Camera Model & Sony IMX234 Exmor \\
& LG-H831_4.4_5312x2988 (RGB), \\
& LG-H831_1.5_3840x2160 (RGB) \\
\hline Sensor size & f/1.8, 26mm (wide), 1/2.6", Laser AF, 3-axis \\
& OIS \\
& 8 MP, f/2.4, 9mm, 1/3.6", no AF \\
\hline Features & LED flash, panorama, HDR \\
\hline
\end{tabular}

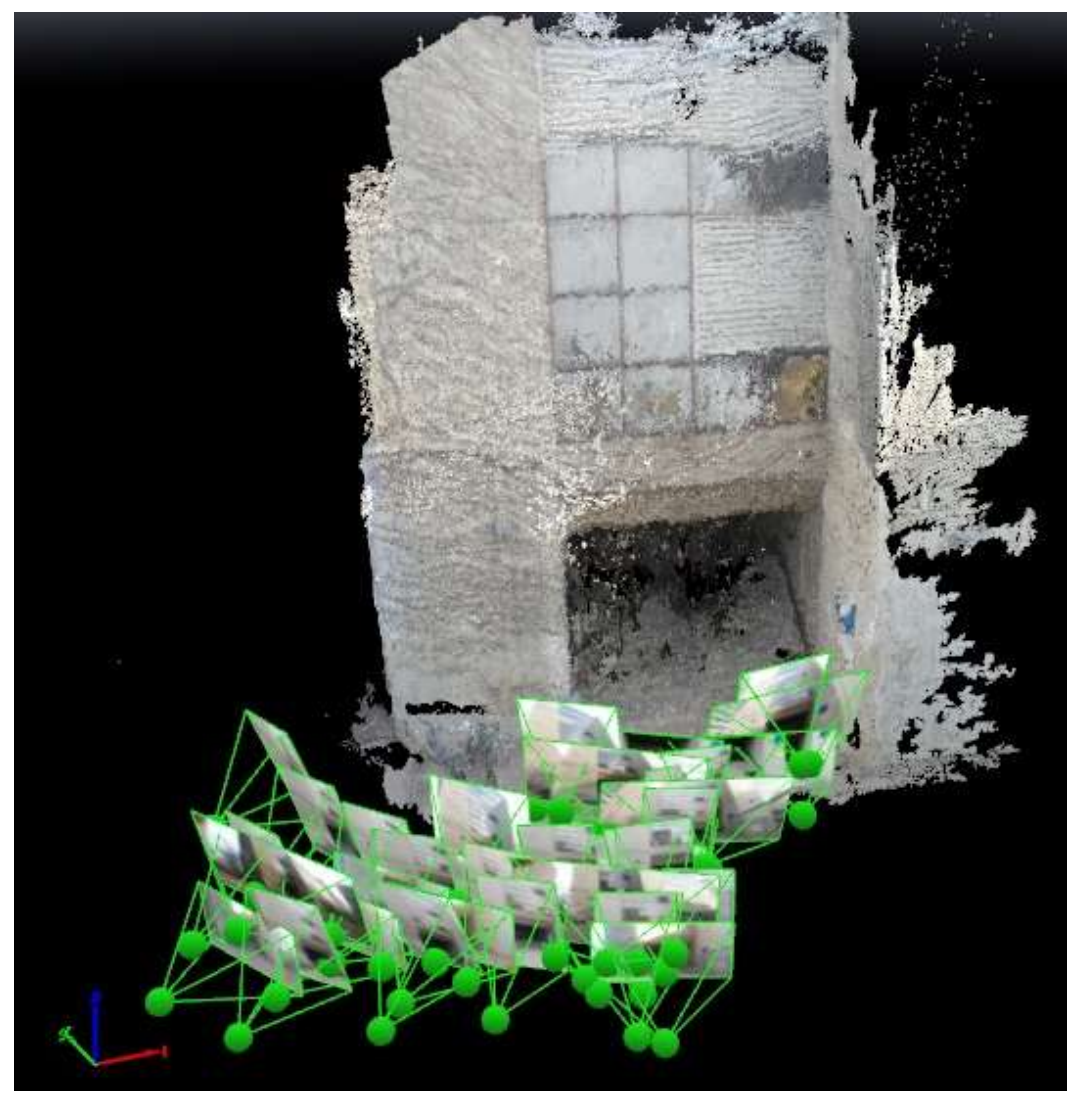

Fig 5.11: EPH façade SfM point cloud (images positions are the green circles) 
Qualitatively assessing the 3D point cloud output from SfM shown in Fig 5.11 shows the general layout and colors of the façade are depicted, however, there are areas that are obviously problematic which can be attributed to the poor lighting or texture conditions for example at the EPH entrance, highlighting the dependability of the model created on the lighting and camera imagery captures conditions. In addition, Quality check parameters for the SfM model processed in the Pix4D processing environment are presented in Tables 5.5. While the bundle block adjustment results is presented in Table 5.6. Table 5.7 presents the number of 3D points calculated from $2 \mathrm{D}$ key points matches. Visiting the values presented in the three tables indicates an adequate resultant model without alarming values as defined in the Pix4D processing environment. A visualization of the average automatic tie points ATP pixel re-projection error magnitude and direction is presented in Fig 5.12.

Table 5.5: SfM Quality check

\begin{tabular}{|l|l|}
\hline Images & 42 out of 44 images calibrated (95\%) \\
\hline Dataset & median of 22639 key points per image \\
\hline Camera Optimization & $\begin{array}{l}2.76 \% \text { relative difference between initial and } \\
\text { optimized internal camera parameters }\end{array}$ \\
\hline Matching & median of 5814 matches per calibrated image \\
\hline
\end{tabular}

Table 5.6: Bundle block adjustment results

\begin{tabular}{|l|l|}
\hline $\begin{array}{l}\text { Number of 2D Key point Observations for } \\
\text { Bundle Block Adjustment }\end{array}$ & 273823 \\
\hline $\begin{array}{l}\text { Number of 3D Points for Bundle Block } \\
\text { Adjustment }\end{array}$ & 108700 \\
\hline Mean Re-projection Error [pixels] & 0.325 \\
\hline
\end{tabular}

Table 5.7: 3D points calculated from 2D key points matches

\begin{tabular}{|ll|ll|l|}
\hline \multicolumn{2}{|l|}{ Number of 3D Points Observed } \\
\hline In 2 Images & 81432 & In 12 Images 139 & In 22 Images & 5 \\
In 3 Images & 16514 & In 13 Images 119 & In 23 Images & 6 \\
In 4 Images & 4805 & In 14 Images 80 & In 24 Images & 3 \\
In 5 Images & 2237 & In 15 Images 41 & In 25 Images & 3 \\
In 6 Images & 1242 & In 16 Images 27 & In 26 Images & 4 \\
In 7 Images & 798 & In 17 Images 20 & In 27 Images & 2 \\
In 8 Images & 448 & In 18 Images 19 & In 28 Images & 1 \\
In 9 Images & 318 & In 19 Images 12 & In 30 Images & 1 \\
In 10 Images & 220 & In 20 Images 10 & In 32 Images & 1 \\
In 11 Images & 186 & In 21 Images 7 & & \\
\hline
\end{tabular}




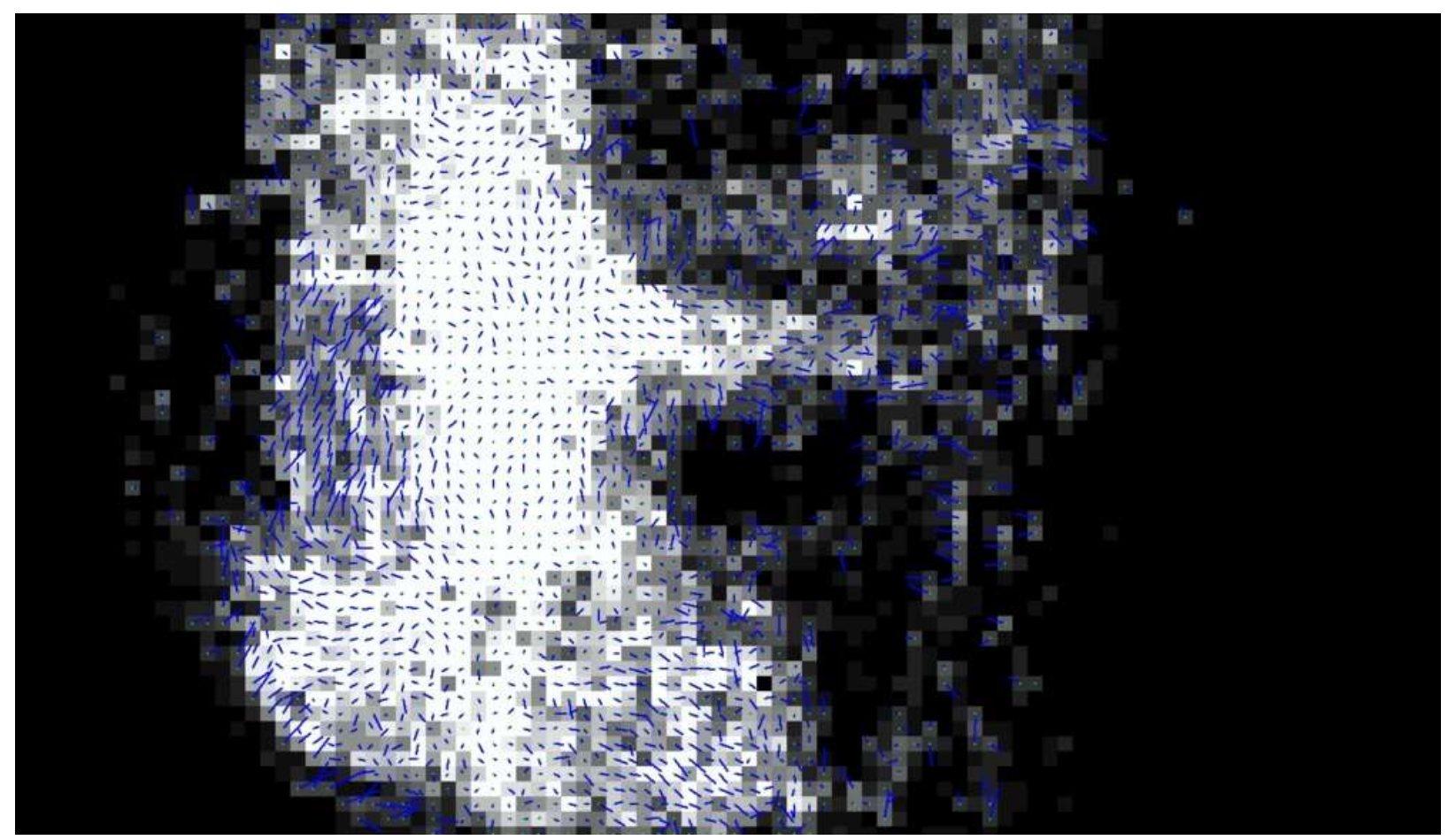

Fig 5.12: Average automatic tie points ATP (darker color represents less points found)

Figure 5.12 shows the number of ATPs per pixel, averaged over all images of the camera model. The image is color-coded between black and white. White indicates that, on average, more than 16 ATPs have been extracted at the pixel location, while black indicates that, on average, 0 ATPs have been extracted. A larger dark portion indicates less ATP and a less optimal 3D calibrated model, which is associated with sub-optimal imagery conditions. As will be shown later with the SfM model in airborne mode, the geolocation information through the imagery EXIF geotags aids in the $3 \mathrm{D}$ reconstruction of the environment. The strength of the ATP matches and the camera positing uncertainty is highlighted in Fig. 5.13. 


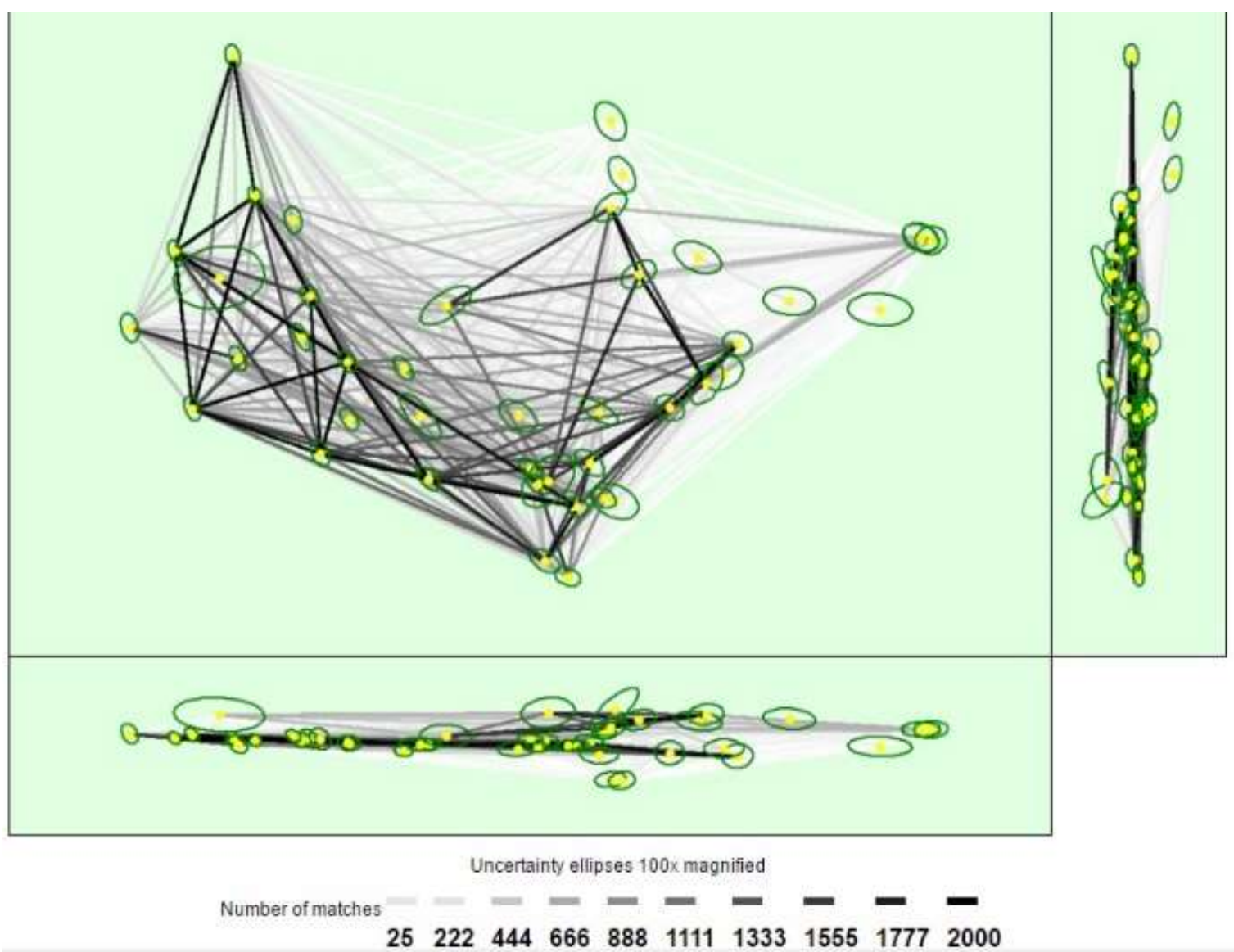

Fig 5.13: 2D key points matches

Figure 5.13 shows the strength of the matches between the images through the number of point matches; specifically, the darker the link, the more point matches are present. On the other hand, dark green ellipses indicate the relative camera position uncertainty of the bundle block adjustment. The light color links represent the need for manual tie point additions or to acquire more images. The figure highlights the inversely proportional relation between the number of matches and the uncertainty of the camera position solution from the SfM workflow. This emphasizes the dependency of the SfM-resultant model on the key point matches between the images. Note that, due to the close proximity of the image exposure stations and the unreliable GPS readings from the cell phone used, the GPS readings were not incorporated into the SfM model derivation and thus the resultant model is up to scale. This would be a disadvantage if measurements needed to be taken from the resultant model.

The dependency of the resultant point cloud on the key point matches between the images reemphasizes one of the SfM's disadvantageous characteristics. In addition, unless the scale is constrained by GPS or a known measured distance, the output is up to scale. It is, nevertheless, a cheap alternative and has a true color visualization which might appeal to wider end-user segments. On the other hand, in using the new LiDAR-based system, the 3D geometry is directly captured but that it lacks the texture and color information. Thus, the fusion between both will mitigate the drawbacks of each approach. 


\subsection{System in Handheld Mode}

The promising results acquired with the usage of the very low-cost yostlabs AHRS and the GPS 18x LVC sensor in stationary mode motivated to test the system in handheld moving mode. A portion of Gould Street near the Church and Gould streets intersection on the Ryerson University campus, Toronto, Canada, has been mapped. Figures 5.14 and 5.15 show a satellite image of the area and a street-view image, respectively, as depicted from Google Earth. The area features trees, building facades with different geometries and building materials, light poles, and parked vehicles

The system setup was similar to the one used in the stationary mode of operation with the yostlabs AHRS and the Garmin GPS 18x LVC sensor attached to the top of the VLP-16. A virtual coordinate system for the AHRS was defined to align with the VLP-16 axes directions. Further, the VLP-16 firing synchronized and time-stamped with GPS position was linked to the AHRS recorded attitudes through the aid of the NTP time synchronization.

Being light-weight and compact, the system was easily mounted on a small handheld camera tripod. The pitch angle of the system (nose up-down around the sensor X-axis) has been incrementally changed to densify the resultant point cloud and increase the total VFOV. The recorded GPS positions in latitude- longitude format has been transformed to a local-level frame of reference through an intermediate transformation to a Cartesian Earth-centered, Earth-fixed ECEF coordinate system. Equation 4.2 has been applied to calculate the points scanned referenced to the VLP-16 reference frame, while DG equation was applied after incorporating the timedependent attitudes and position data recorded by the AHRS and the GPS sensor. The resultant point cloud is shown in Fig. 5.16. As anticipated, with the imprecise low-cost sensors used for the DG of the LiDAR point cloud, features are unrecognizable within the resultant point cloud. Nevertheless, applying the newly developed workflow allows examining each LiDAR data frame individually revealing a clearer depiction of the features within the resultant point cloud, as shown in Fig 5.17. 


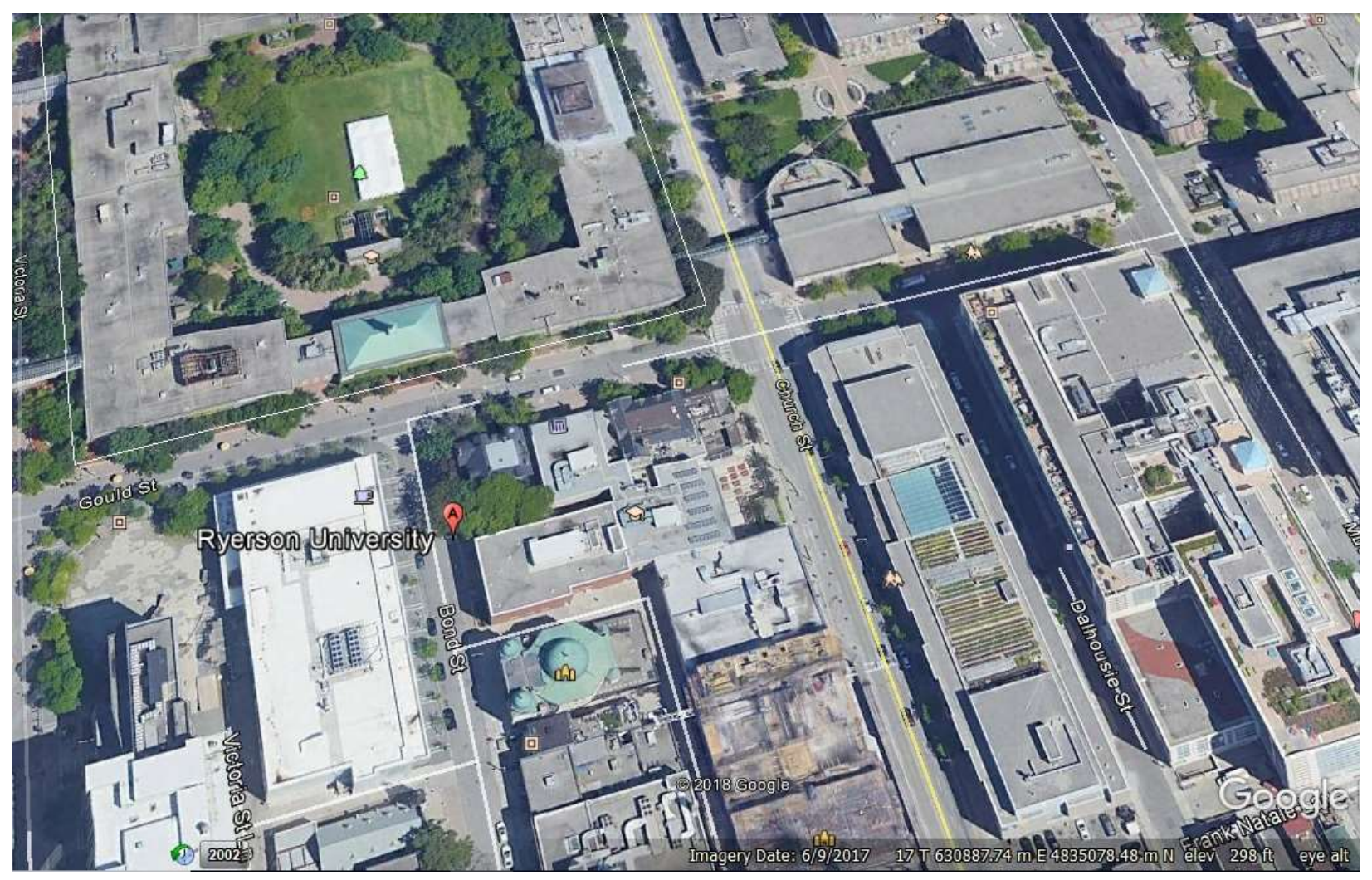

Fig 5.14: A satellite image; Gould St and Church St intersection

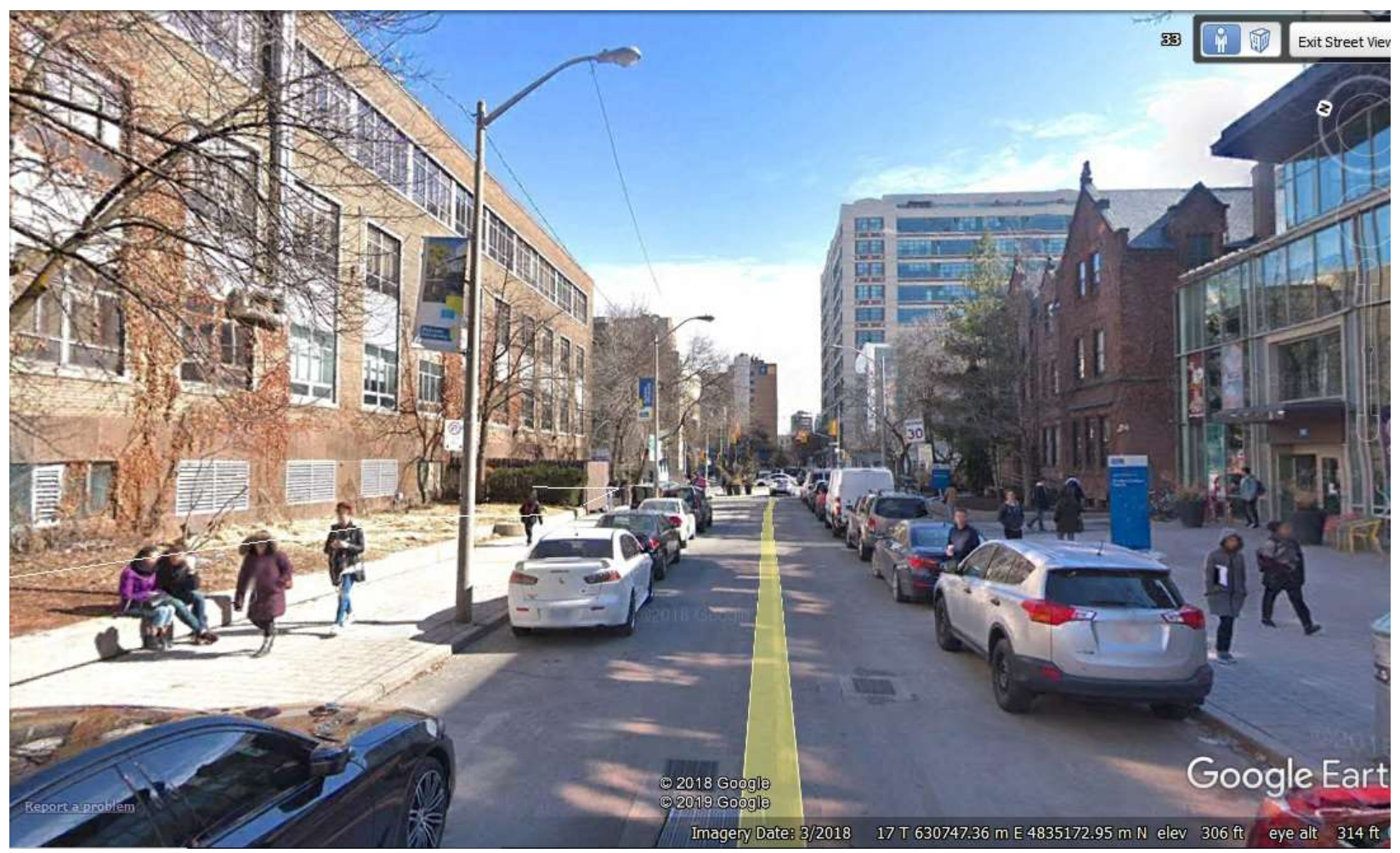

Fig 5.15: A street-view image; Gould St and Church St intersection 


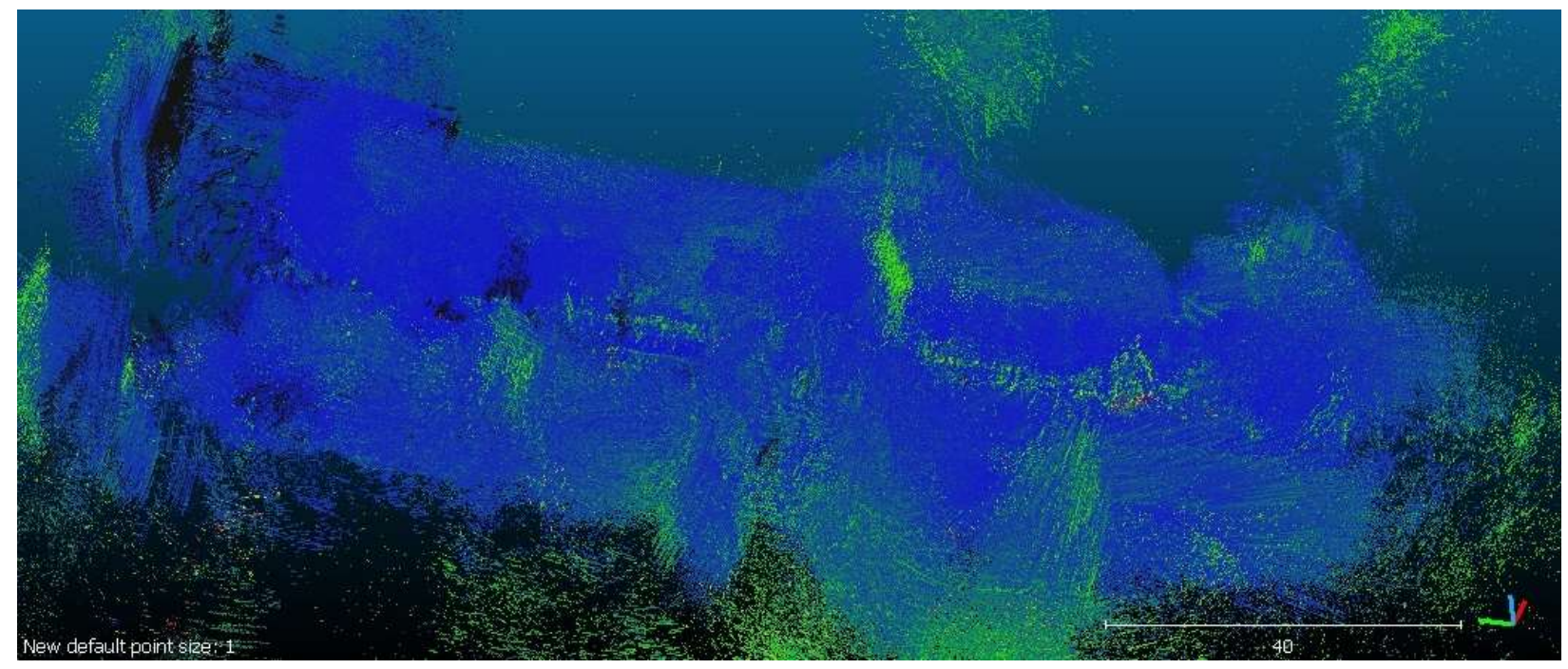

Fig 5.16: Gould St mapped with yostlabs AHRS and Garmin GPS 18x LVC (All frames)

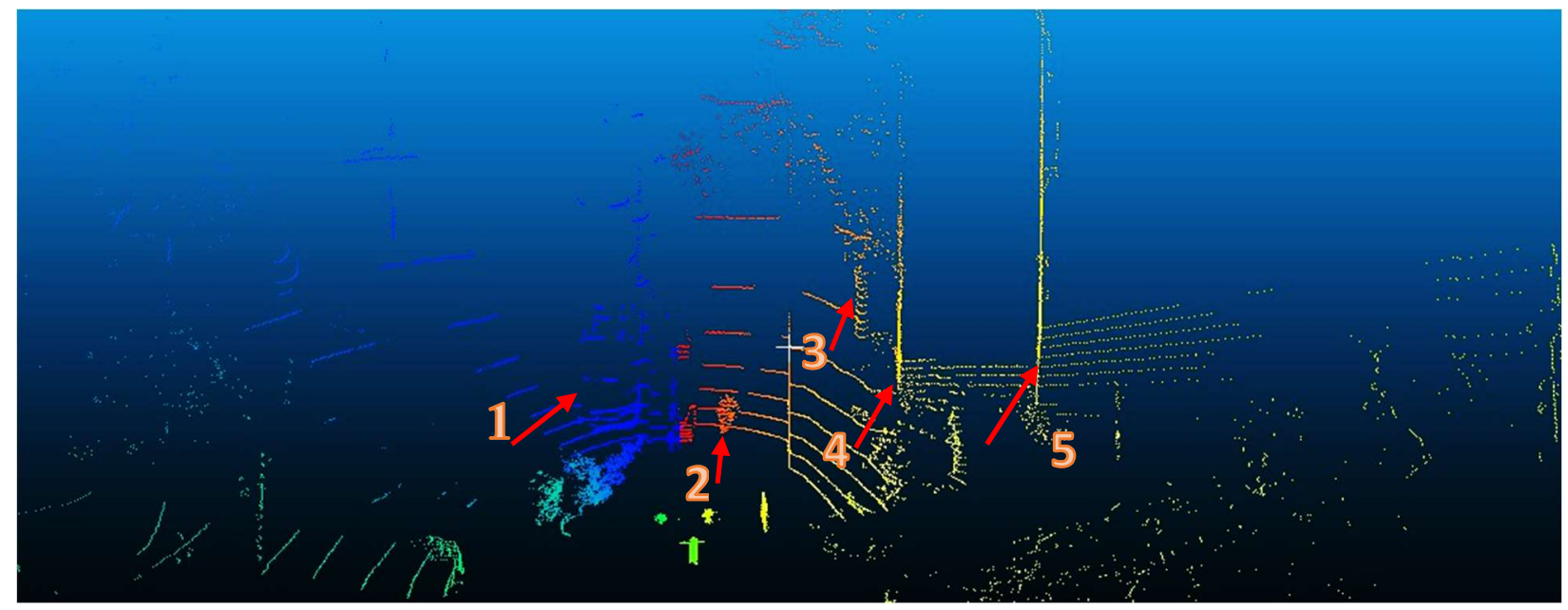

Fig 5.17: Gould St mapped with yostlabs AHRS and Garmin GPS 18x LVC (One frame)

The results depicted in Fig. 5.16 and the frames' misalignment may be explained by the following factors, namely, the imprecise measurements of the sensor attitudes (but more critically the imprecise measurements of the sensor position) are due to the very low-cost sensors used, coupled with multiple readings from the VLP-16 for almost the same area due to its scanning mechanism and the sensor horizontal orientation mounting. The utilization of the new workflow of data fusion on the SW level allowed for examining up to one frame of the resultant georeferenced point cloud, revealing a clear depiction of the features, as shown in Fig. 5.17. A parked vehicle is labeled as object number 1 , a person is object number 2, a tree is object number 3 , the fence corner is shown as object number 4 , and the building corner is successfully mapped as object number 5 . Frames number 50 and 100 are depicted in Fig. 5.18 to show the misalignment of the same objects due to the imprecise position and attitude measurements in addition to the boresight and lever arm 
misalignment effect, which can be alleviated with a calibration data collection mission.

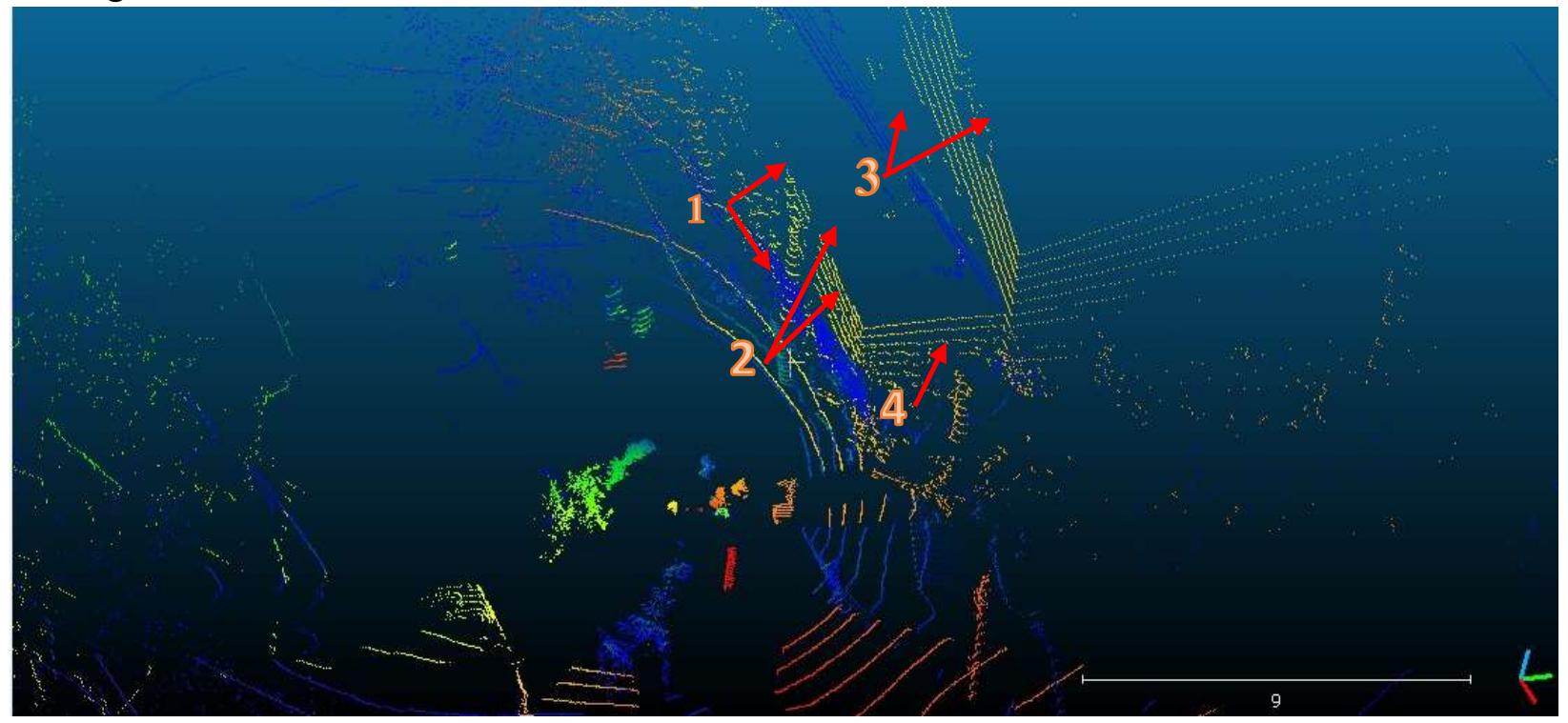

Fig 5.18: Gould St mapped with yostlabs AHRS and Garmin GPS 18x LVC Frames; \#50(blue) $\& 100$ (colored)

As shown in Fig. 5.18, the objects that are remapped from different sensor positions and attitudes are misaligned due to the low precision associated with the low-cost sensors used and the VLP-16 multiple scanning of the same object. Examples of such objects are: a tree (object number 1), the side of a fence (object number 2), and the side of building (object number 3 ). On the other hand, a tree (object number 4) was only mapped once from the frame number 50. As it was out of the scanning view of frame number 100, it is only mapped once and can be clearly depicted. Taking into consideration the thousands of frames recorded by the VLP-16 and the misaligning of objects which occurs, we can better understand the results shown in Fig. 5.16. The ability to analyse each frame of the LiDAR data georeferenced through the new approach of frame discretization proposed and implemented in this research for the DG process of LiDAR data adds full control to the operator to better interpret the results at hand and to select the level of details and abstraction needed for a specific application purpose. Figures 5.19 and 5.20 show different levels of details and abstraction of the area by using every other 50th frame, and every other 100th frame of the georeferenced data. 


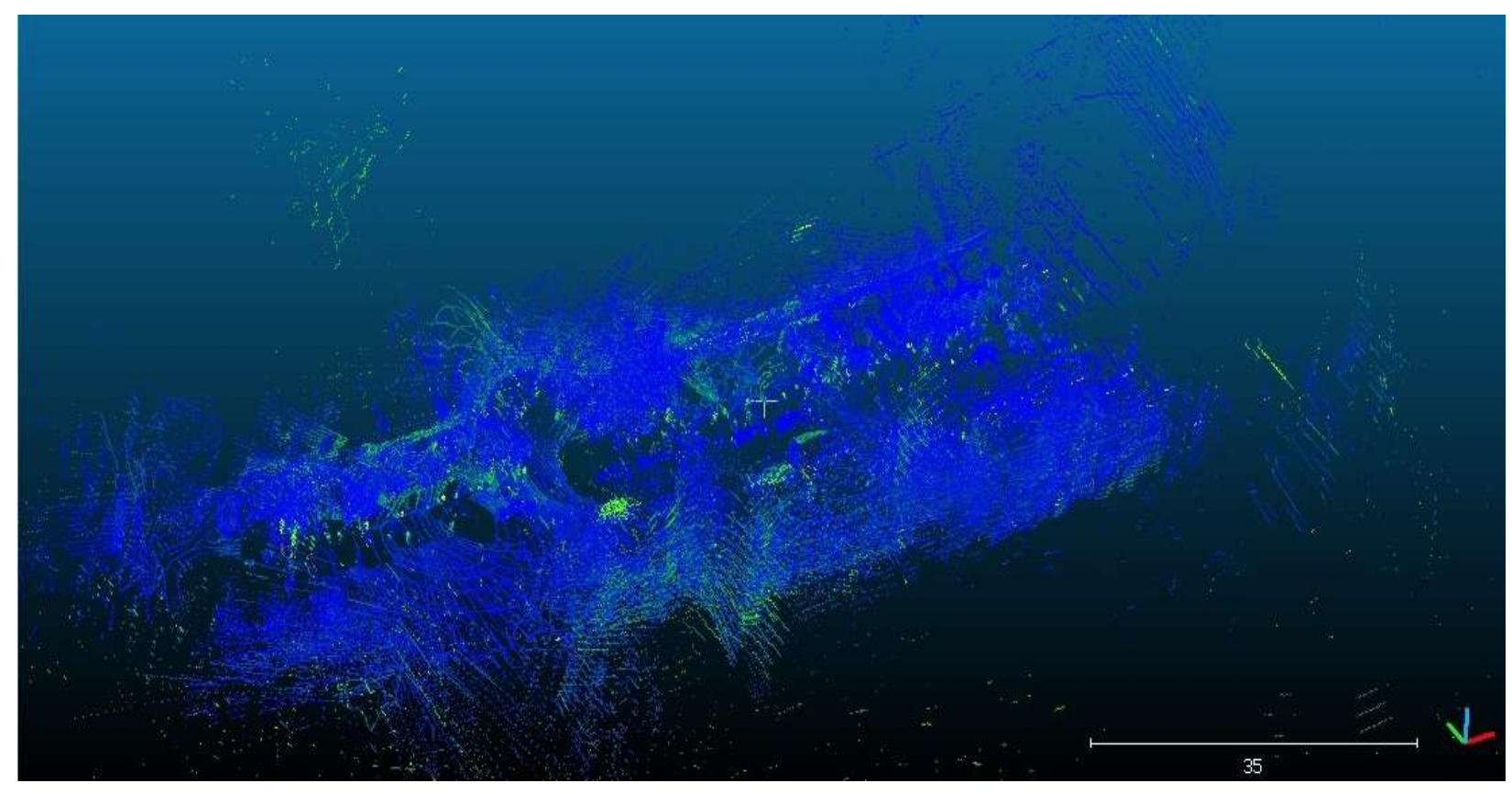

Fig 5.19: Gould St mapped with yostlabs AHRS and Garmin GPS 18x LVC (Every other 50th frame)

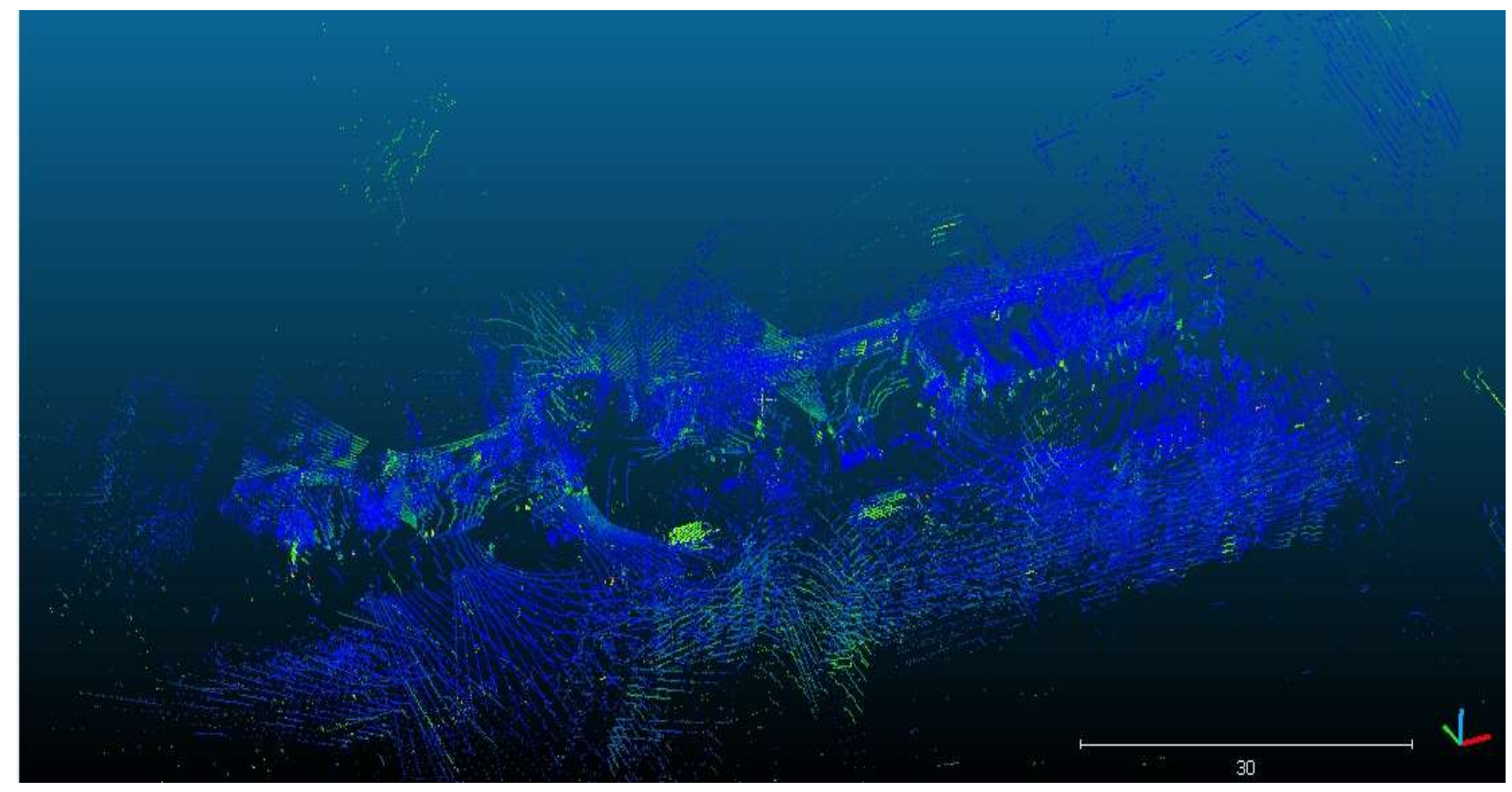

Fig 5.20: Gould St mapped with yostlabs AHRS and Garmin GPS 18x LVC (Every other 100th frame) 
The new SW integration workflow, as explained in section 3.4, allowed for control up to the sensor's single sweep, adding flexibility to the abstraction and level of details that can be utilized. As shown in Figs. 5.19 and 5.20, the level of detail and the point cloud density vary according to the number of frames used in the area mapping. This level of control and flexibility in the output data abstraction is made possible by applying the new SW integration workflow.

In order to decrease the misalignment between the different frames mapped, a new incremental frame-to-frame registration applying the Iterative Closest Point (ICP) algorithm is implemented. To decrease the effect of the noise within the point cloud data and its negative effect on the ICP algorithm, a statistical outlier removal filter is applied to the data in advance of the ICP algorithm application. The statistical outlier removal filter incorporates the information from the neighbouring points, as it measures the distance to the surrounding points using filter criteria presented in Equation 5.1. The ICP algorithm is then recursively applied to successive frames.

$$
D_{\text {max }}=\mu_{d}+n * \sigma
$$

Where

$$
\begin{aligned}
& D_{\text {max }} \text { is the max distance } \\
& \mu_{d} \quad \text { is the mean distance } \\
& n \quad \text { is the n. sigma to be used for the filtering } \\
& \sigma \quad \text { is the standard deviation }
\end{aligned}
$$

To speed up the processing applied on the point cloud data, which can easily reach millions of points, octree portioning of the point cloud is applied beforehand. A summary of the ICP algorithm is presented according to [101], as follows. The ICP is a simple yet effective method for point cloud registration. An initial good alignment of the point clouds is required in order for the ICP to perform adequately. The initial alignment is achieved through the direct georeferencing. Hence, the problem of point cloud registration can be defined as an optimization problem aimed at finding the best transformation that aligns the target dataset to the reference dataset. Let $\mathrm{P}_{\mathrm{A}}$ be the points of the target dataset defined in an A coordinate system, and let $\mathrm{Q}_{\mathrm{B}}$ be the points of the reference dataset defined in a $\mathrm{B}$ coordinate system. The aim of the registration is to minimize an error function in order to find the best required transformation of $\mathrm{T}\left(\mathrm{P}_{\mathrm{A}}\right)$ to $\mathrm{Q}_{\mathrm{B}}$. Thus, the transformation (Equation 5.2) can be expressed as:

$$
T_{A}^{B}=\operatorname{argmin}_{T}\left(\operatorname{error}\left(T\left(P_{A}\right), Q_{B}\right)\right)
$$

The error function is computed on the match between point pairs. Let the match

$$
M=\operatorname{match}(P, Q)=\{(p, q): p \in P, q \in Q\}
$$


The match is done on the bases of the closest point. Thus the error function can be defined as

$$
\operatorname{error}(P, Q)=\sum_{(p, q) \epsilon M} d(p, q)
$$

The outliers are identified and removed through the pre-applied statistical outlier removal filter to make the error function more robust. The ICP iterates through transformation, minimizing the error every run and thereby enhancing the matching process. In this way, the ICP builds a sequence of transformations. Let iteration ${ }_{i-1}^{i} T$ indicate the sequence of iteration. From the points' relative positions, a new match Mi is formed and a new transformation ${ }_{i}^{i+1} T$ is calculated by minimizing the error:

$$
{ }_{i}^{i+1} T \Longleftarrow \operatorname{argmin}_{T}\left(\operatorname{error}\left(T\left(P_{A}\right), Q_{B}\right)\right)
$$

The required transformation is the composition of the intermediate transformations

$$
T_{A}^{B}=\left(\Pi_{i} \quad{ }_{i-1}^{i} T\right) T_{\text {init }}
$$

Where $\left(\Pi_{i}{ }_{i-1}^{i} T\right)$ is the composition of intermediate transformations and $T_{\text {init }}$ is the initial transformation.

Applying the incremental frame-to-frame registration as described above reduces the misalignment between the different frames, as illustrated with the whole data frames in Fig. 5.21. The georeferenced point cloud shown in the figure depicts the mapped area mapped as the augmented version of the georeferenced point cloud shown in Fig. 5.16, after the application of the incremental frame-to-frame registration. A clearer depiction of the details within the area mapped is thus achieved. 


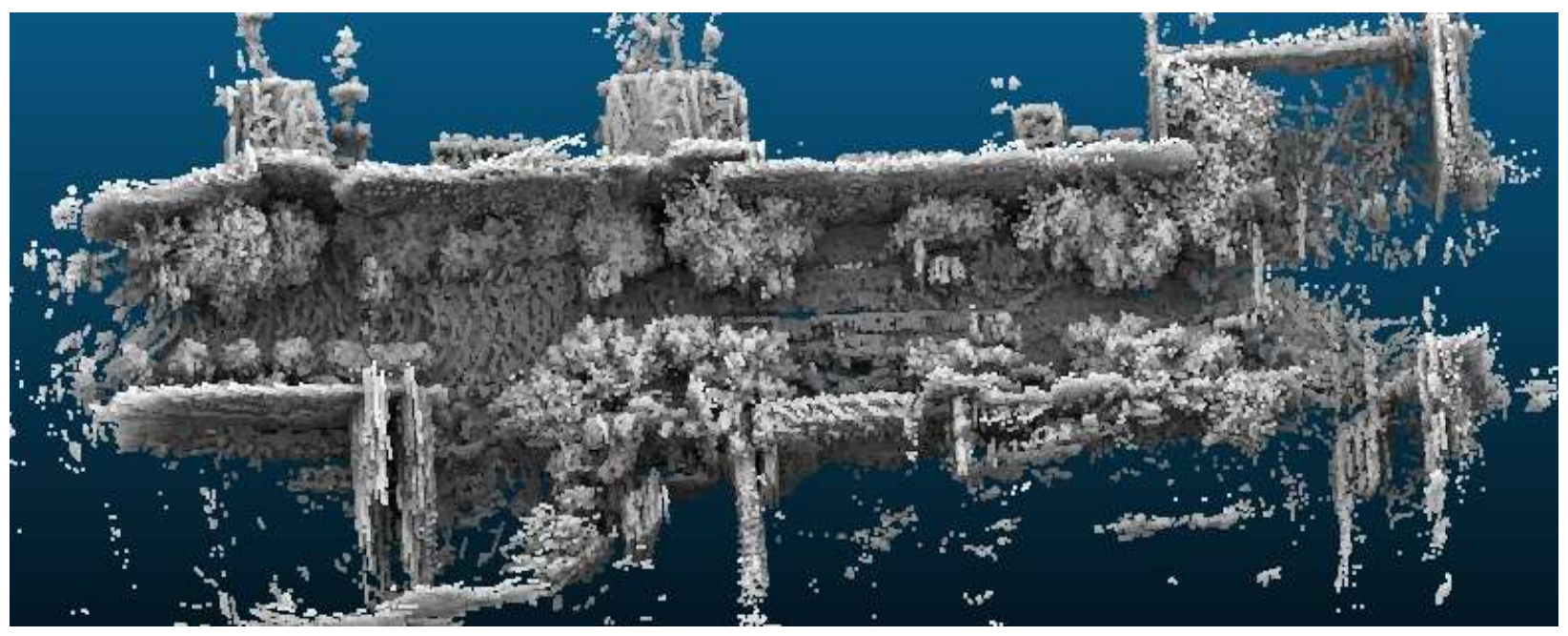

Fig 5.21: Gould St mapped with yostlabs AHRS and Garmin GPS 18x LVC

Enhanced with the recursive ICP application

To obtain a quantitative assessment of the mapped area, the trajectory has been superimposed on accurate LiDAR data captured through an aerial flying mission covering the Greater Toronto Area (GTA). The summary of the aerial mission is presented in Table 5.8.

Table 5.8: The summary of the Aerial LiDAR mission used for the quantitative validation

\begin{tabular}{|c|c|c|c|c|c|c|c|c|c|}
\hline \multicolumn{10}{|c|}{ Acquisition Parameters } \\
\hline $\begin{array}{c}\text { Date } \\
\text { (MM/DD/YY) }\end{array}$ & Mission & $\begin{array}{c}\text { Flying } \\
\text { Height } \\
(\mathrm{m})\end{array}$ & $\begin{array}{c}\text { Flying } \\
\text { Speed } \\
\text { (knots) }\end{array}$ & \begin{tabular}{|c|} 
Pulse \\
Rate \\
Rep \\
(kHz) \\
\end{tabular} & $\begin{array}{l}\text { Scan } \\
\text { Freq } \\
(\mathrm{Hz}) \\
\end{array}$ & $\begin{array}{c}\text { Scan } \\
\text { Angle } \\
\text { (degree) }\end{array}$ & $\begin{array}{c}\text { Side } \\
\text { Lap } \%\end{array}$ & $\begin{array}{c}\text { Point } \\
\text { Density } \\
\left(\mathrm{pts} / \mathrm{m}^{2}\right)\end{array}$ & LiDAR System \\
\hline $04 / 06 / 15$ & $7915096 a$ & 1300 & 160 & 400 & 52 & 40 & 50 & 10.0 & Leica ALS70 \\
\hline $04 / 07 / 15$ & $7915097 a$ & 1300 & 160 & 400 & 52 & 40 & 50 & 10.0 & Leica ALS70 \\
\hline $04 / 11 / 15$ & $7915101 a$ & 1300 & 160 & 400 & 52 & 40 & 50 & 10.0 & Leica ALS70 \\
\hline $04 / 25 / 15$ & $7915115 b$ & 1300 & 160 & 400 & 52 & 40 & 50 & 10.0 & Leica ALS70 \\
\hline \multicolumn{3}{|c|}{ Multiple Return Capabilities: } & \multicolumn{2}{|c|}{ YES } & \multicolumn{4}{|c|}{ Number of returns recorded: } & Maximum 4 \\
\hline \multicolumn{10}{|c|}{ Geodetic Control } \\
\hline \multicolumn{2}{|c|}{ Horizontal Datum: } & \multicolumn{3}{|c|}{ Nad83 CSRS } & \multicolumn{3}{|c|}{ Vertical Datum: } & \multicolumn{2}{|r|}{ CGVD28 } \\
\hline \multicolumn{2}{|c|}{ Geoid Model: } & \multicolumn{3}{|c|}{$\mathrm{HT} 2.0$} & \multicolumn{3}{|c|}{ UTM Zone: } & \multicolumn{2}{|r|}{17} \\
\hline \multicolumn{10}{|c|}{ Note: We established a local geodetic network fixed to the following control: } \\
\hline \multicolumn{2}{|c|}{ Station ID } & \multicolumn{3}{|c|}{ Lat } & \multicolumn{3}{|c|}{ Long } & \multicolumn{2}{|c|}{ Ellp Height } \\
\hline \multicolumn{2}{|c|}{61313} & \multicolumn{3}{|c|}{$4346 \quad 05.44812$} & \multicolumn{3}{|c|}{-793849.15723} & \multicolumn{2}{|c|}{154.971} \\
\hline \multicolumn{2}{|c|}{653196} & \multicolumn{3}{|c|}{433530.99772} & \multicolumn{3}{|c|}{-793611.54776} & \multicolumn{2}{|r|}{92.610} \\
\hline
\end{tabular}

Airborne Imaging performs a complete calibration on every LiDAR acquisition flight, data is acquired over a calibration site flown with at least two passes in opposite directions before and after the flight. Any error in the attitude of the aircraft (roll, pitch and heading) can be observed and corrected for within system specifications. To statistically quantify the accuracy, we compare the LDAR elevations with independently surveyed ground points. A GPS mounted truck collects data while driving on an open road. The kinematic positions on the road are post-processed from a nearby base station (common to the aerial survey)

\begin{tabular}{|c|c|}
\hline Accuracy & \\
\hline Horizontal Accuracy, $95 \%$ or $2 \sigma:$ & $30 \mathrm{~cm}$ \\
\hline Fundamental Vertical Accuracy (on flat hard surfaces), $95 \%$ or $2 \sigma$ : & $10 \mathrm{~cm}$ \\
\hline
\end{tabular}


The dataset used for the quantitative assessment is an aerial LiDAR dataset captured in 2015 by Airborne Imaging, a Canadian (Calgary-based) company. The datasets feature a point density of $10 \mathrm{pts} / \mathrm{m}^{2}$, a reported horizontal accuracy of $30 \mathrm{~cm}$ and vertical accuracy of $10 \mathrm{~cm}$, both reported with $95 \%$ confidence or $2 \sigma$. The horizontal datum is NAD83 CSRS and the vertical datum is CGVD28 with the geoid model HT2.0. The data is projected to the UTM projection zone $17 \mathrm{~N}$.

As expected with the low-cost customer-grade GPS receiver used, the position readings deviate from the accurate positions. The deviations are shown in Fig. 5.22. 


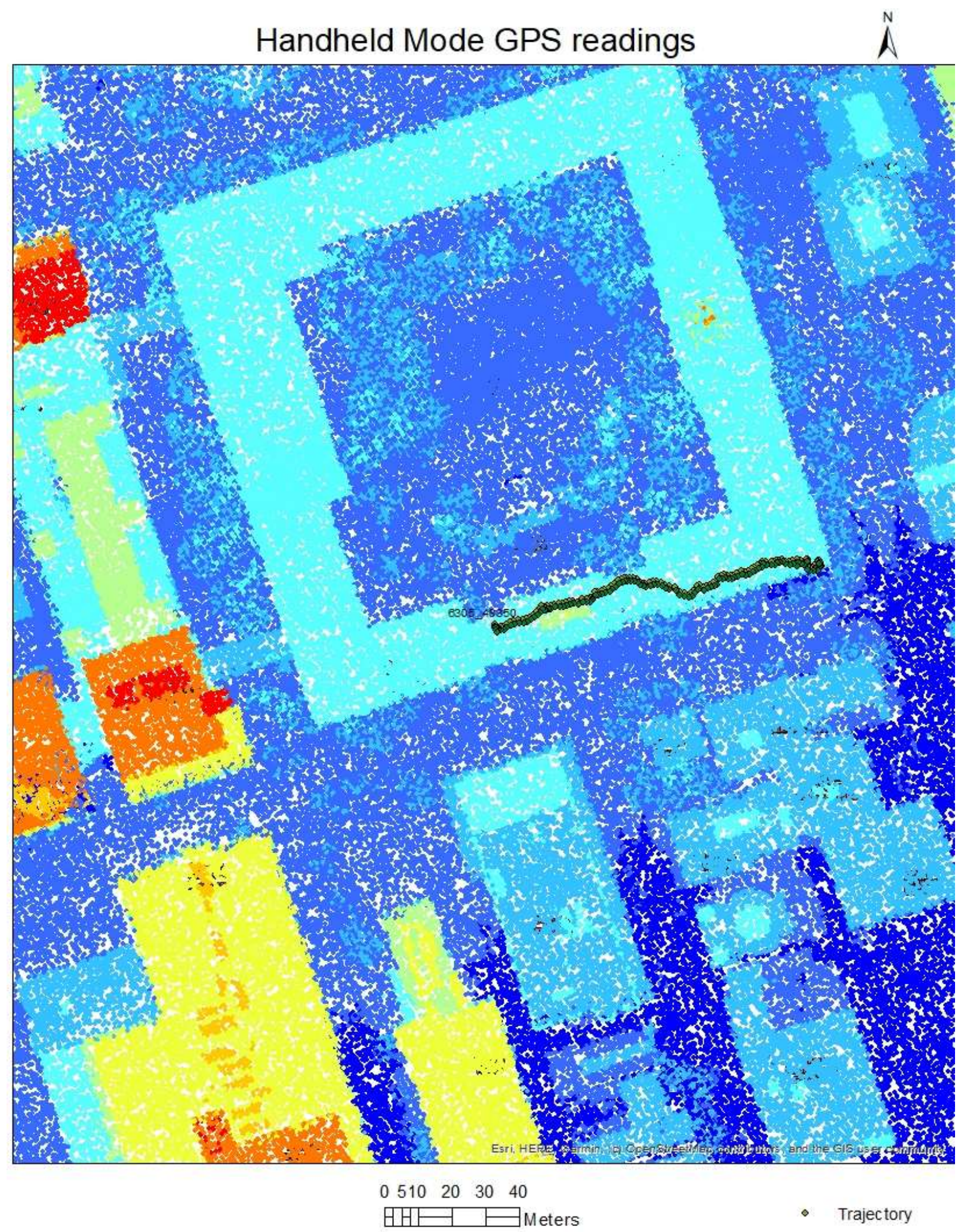

Fig 5.22: Deviation of the GPS readings to the accurate LIDAR ground truth (Aerial captured 3D point cloud, color coded by height) 
Figure 5.22 indicates the positions recorded by the Garmin 18x LVC sensor positions reading. The readings deviate considerably from the accurate ground truth data represented by the accurate aerial LiDAR dataset. The path followed during the data collection has been interpreted and extracted from the aerial LiDAR data and the deviation to the superimposed GPS trajectory has been measured. On average, the deviation is $15 \mathrm{~m}$. Although the Garmin 18x LVC is a customergrade GPS sensor, the expected accuracy should still have been better than the deviation experienced.

The large deviation in the readings may suggest the occurrence of unnoticed EMI while collecting the data. In addition, part of the deviation could be attributed to the different datum used in each of the two datasets, as the Garmin 18x LVC NMEA is referenced to WGS84 geographic, while that of the aerial LiDAR dataset is referenced to NAD83 CSRS datum. Although the absolute geolocation accuracy deviates from the ground truth data, the relative geolocation accuracy is quite adequate, as depicted in the details captured and presented in Fig. 5.21. From a practical perspective, the Garmin 18x LVC sensor could be used only for time-stamping the VLP-16 data.

\subsection{The System as a Mobile Mapping System}

In order to test the system in MMS data collection mode, a data collection mission has been implemented. It is linked to the dynamics associated with using the system on a moving platform. The original setup of the system uses the APX-15 as the POS sensor is used. Interestingly, the APX-15 was originally developed and optimized for a UAV mapping application with an open sky view for the GNSS signal reception. The new utilization of the sensor in an urban environment

with the multipath problem for the GNSS signal reception adds to the challenge of using it within an MMS. Before reflecting on the MMS data collection mode of operation for the newly developed system, a timeline of the system hardware integration versions is highlighted in Fig. 5.23 . 


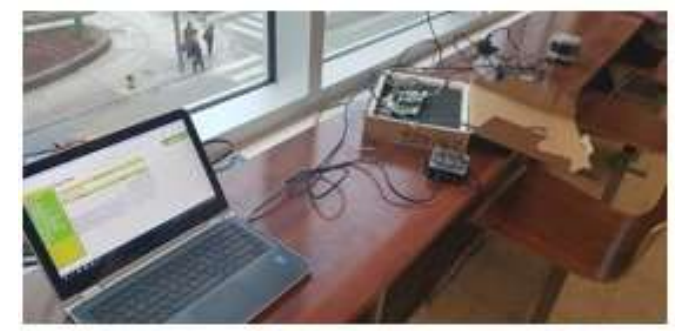

a)

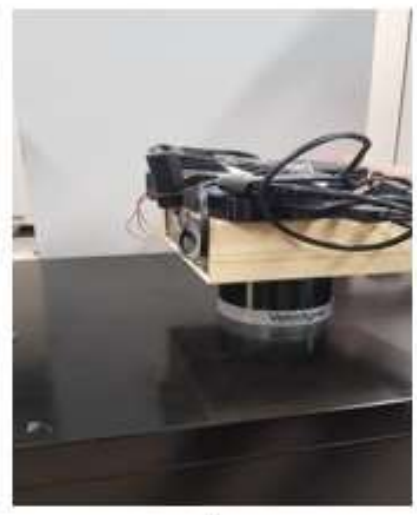

c)

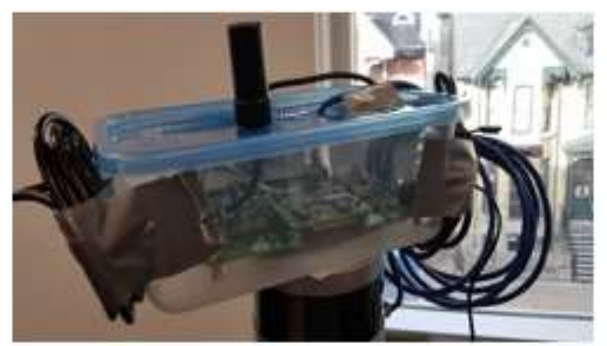

b)

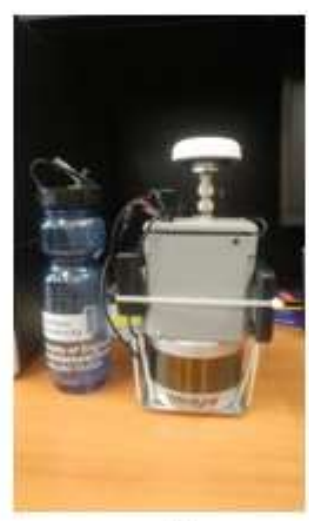

d)

Fig 5.23: Timeline for the hardware integration a) Initial testing, b) Prototype version 1

c) Prototype version 2, d) Self-contained system

Initially, the system mounting on the vehicle relied on a tripod that was extended outwards from the car sunroof panel, as illustrated in Fig. 5.24(a). Subsequently, a more practical and efficient coupling has been achieved by introducing an ultra-light and compact three-arm suction-cup mounting system originally used for DSLR cameras, as shown in Fig. 5.24(b). This practical and efficient mounting system is achievable through the lightweight and small-form factor of the system developed. The new mechanism enables the mounting of the system on any part of a moving platform, which means it no longer has to be mounted on the car roof. Moreover, it does not require a van, as is typically the case with the commercial mobile mapping systems currently available on the market. 


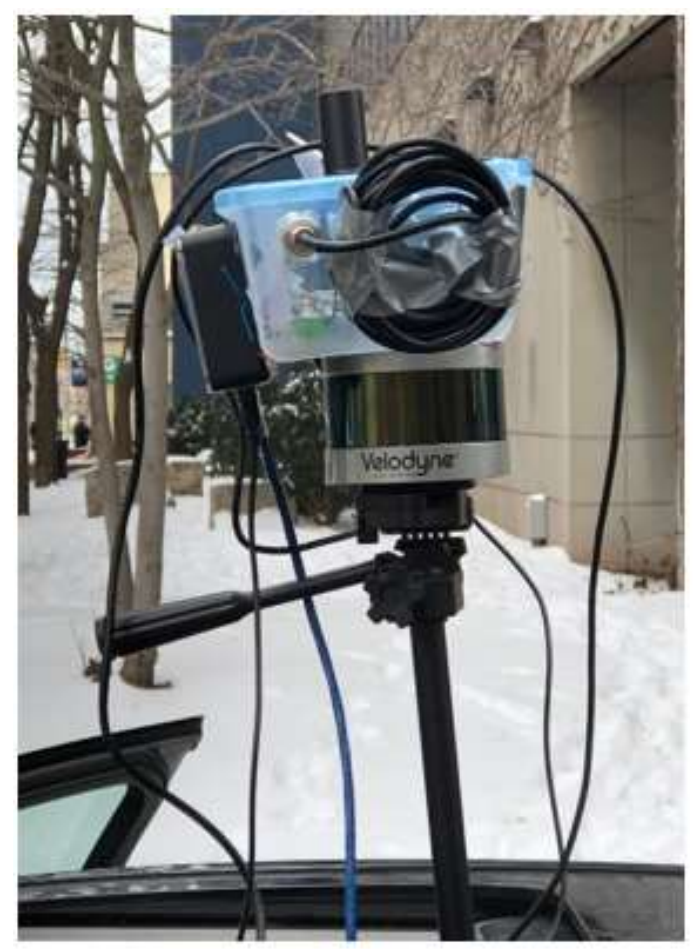

a)

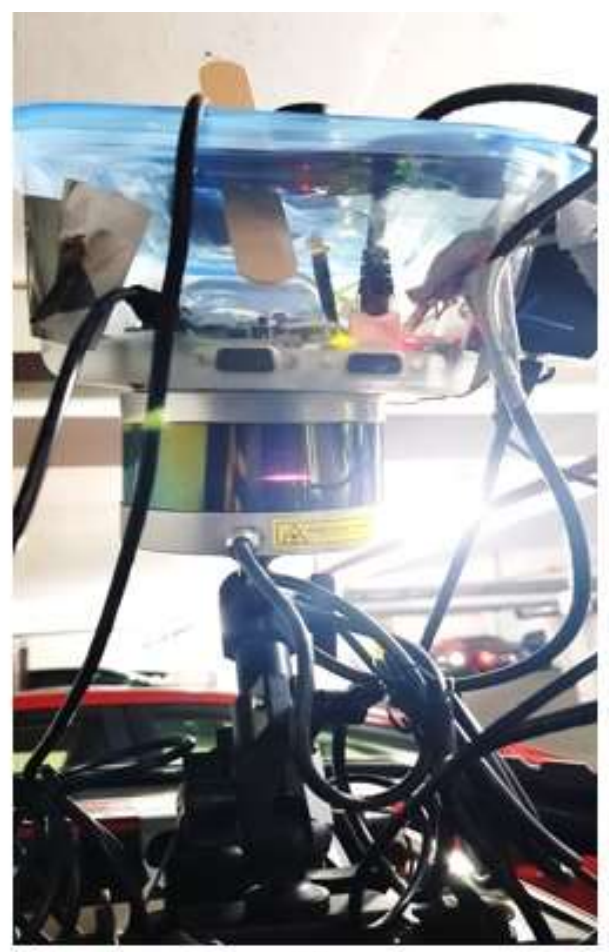

b)

Fig 5.24: System Car mounting a) initial b) practical and efficient

The data collection mission with the developed system deployed as an MMS has been carried out, and a block within the Ryerson University campus has been mapped. The mapped area is depicted through a satellite image from Google earth in Fig 5.25.

A base station was deployed in the middle of the area as shown in Fig 5.25 for around two hours to enhance the accuracy of the post-processing trajectory solution. The Smoothed Best Estimate of Trajectory (SBET) was calculated in a POSPac environment. The workflow depicted in Fig. 3.10 was followed to georeference the resultant point cloud. It is worth noting that the POSPac environment was optimized for the UAV-based mapping application, as the utilization of the APX15 in an MMS as implemented in the present research is a new application for the sensor. 


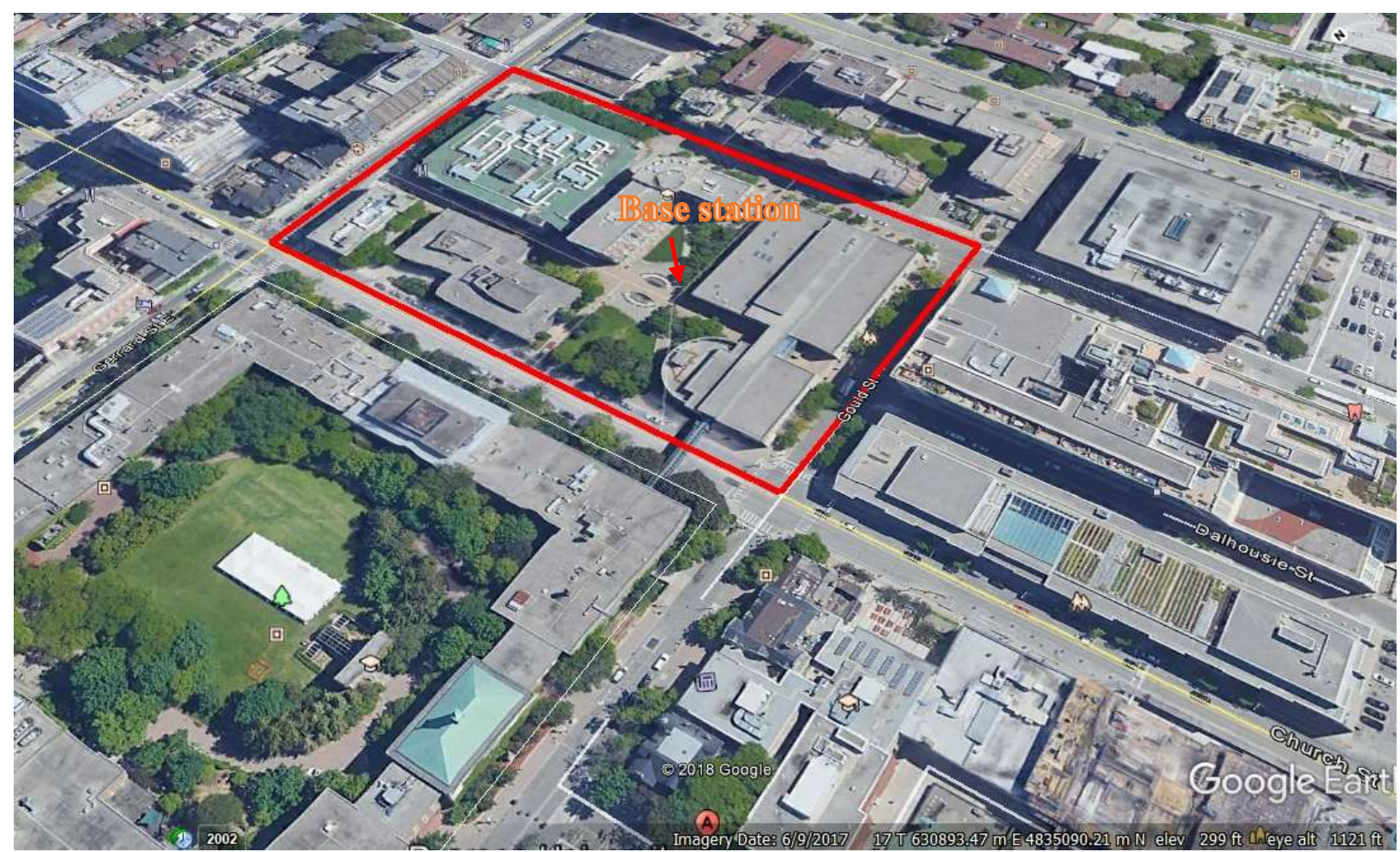

Fig 5.25: The area mapped in MMS mode

To quantitatively assess the output of the trajectory, it was superimposed on an orthophoto depicting the area. The data collection followed two opposite direction movements of the car, as is clearly depicted on the map by the double loops of the trajectory shown in Fig. 5.26. 


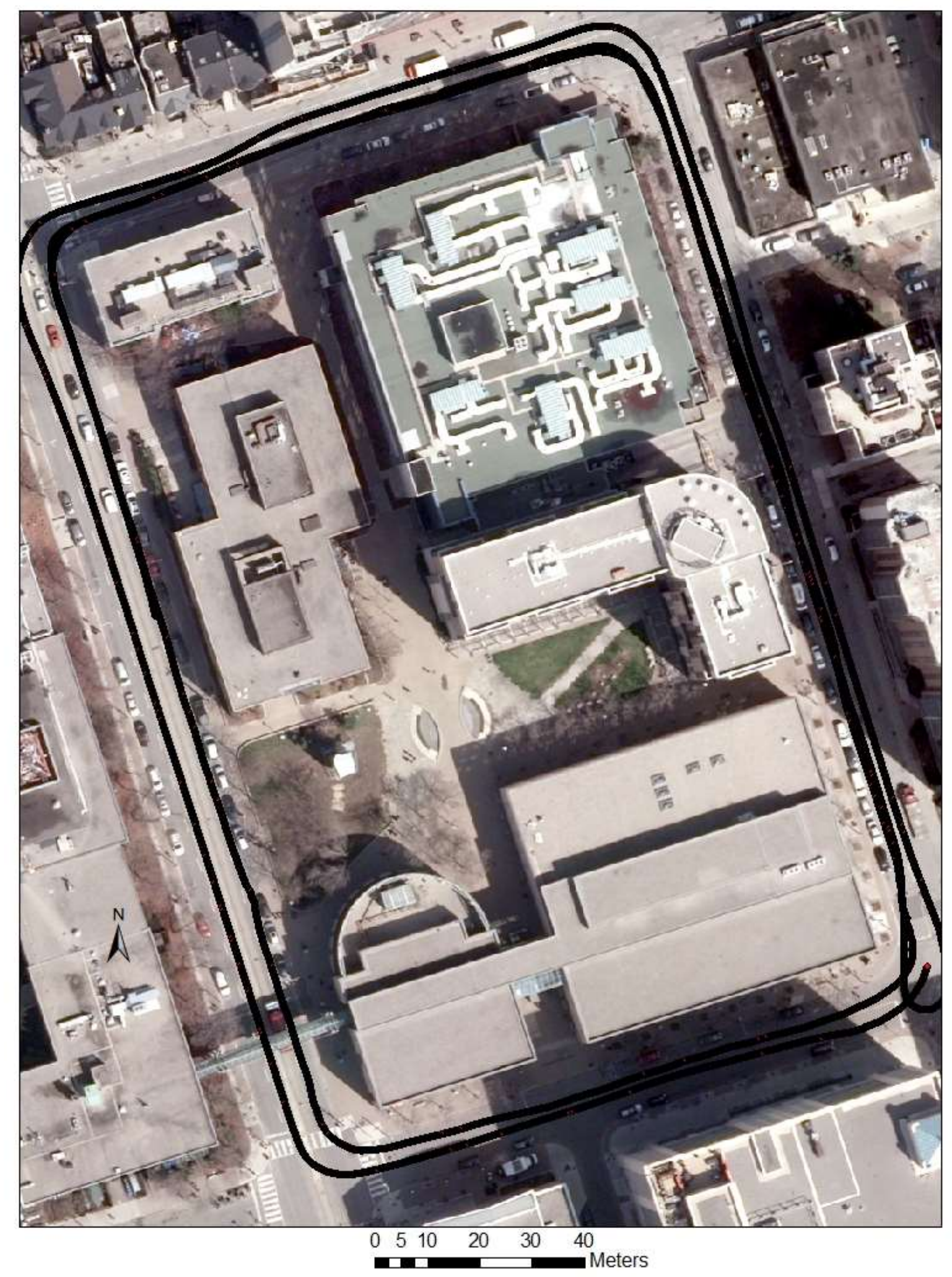

Fig 5.26: Double loops of the trajectory 
The performance metrics for the position and attitude determination from the SBET calculation for the north, east, down position and the roll, pitch and heading attitudes are presented in Figs. 5.27 to 5.32 , respectively.

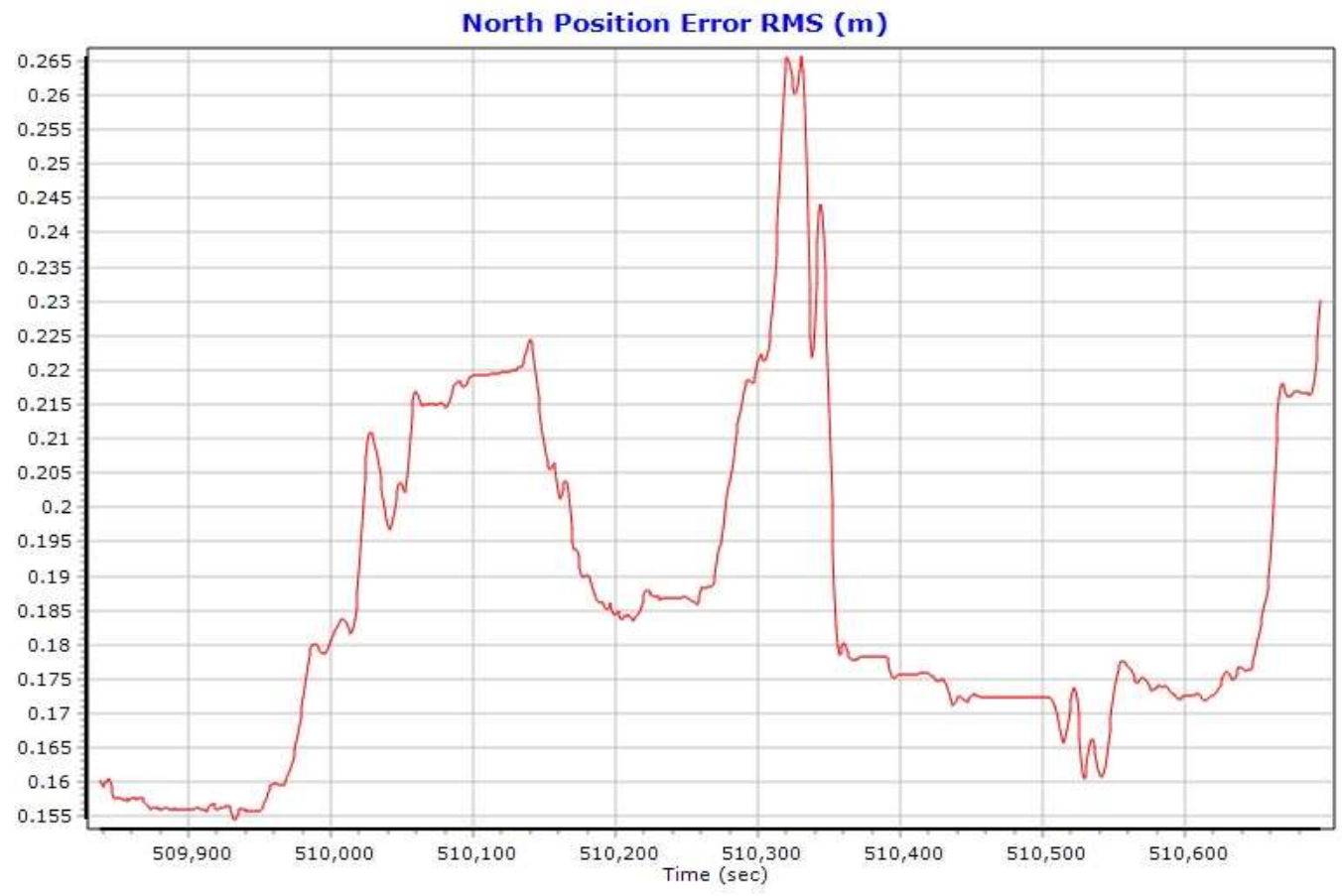

Fig 5.27: MMS SBET North position RMS (m)

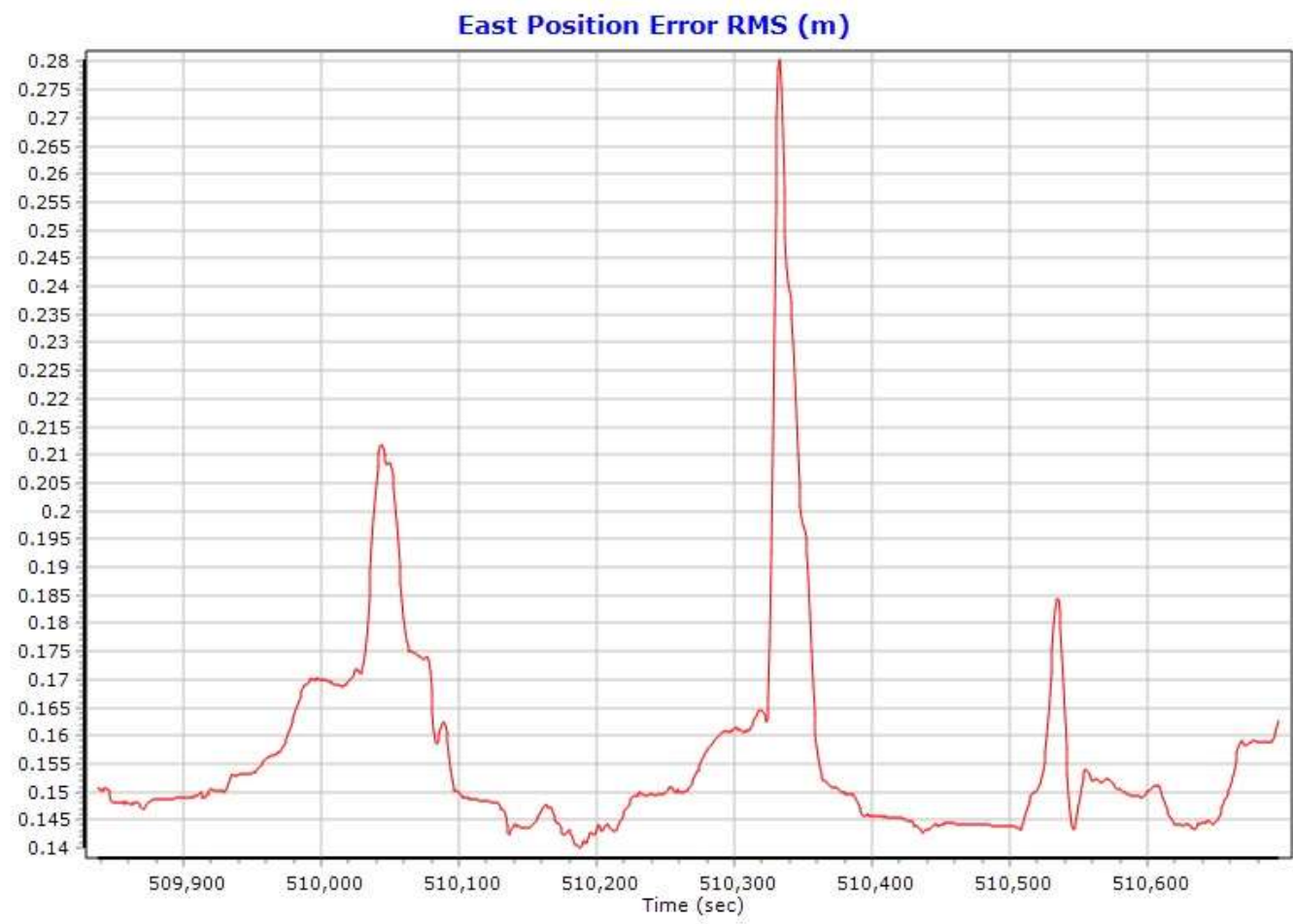

Fig 5.28: MMS SBET East position RMS (m) 
Down Position Error RMS (m)

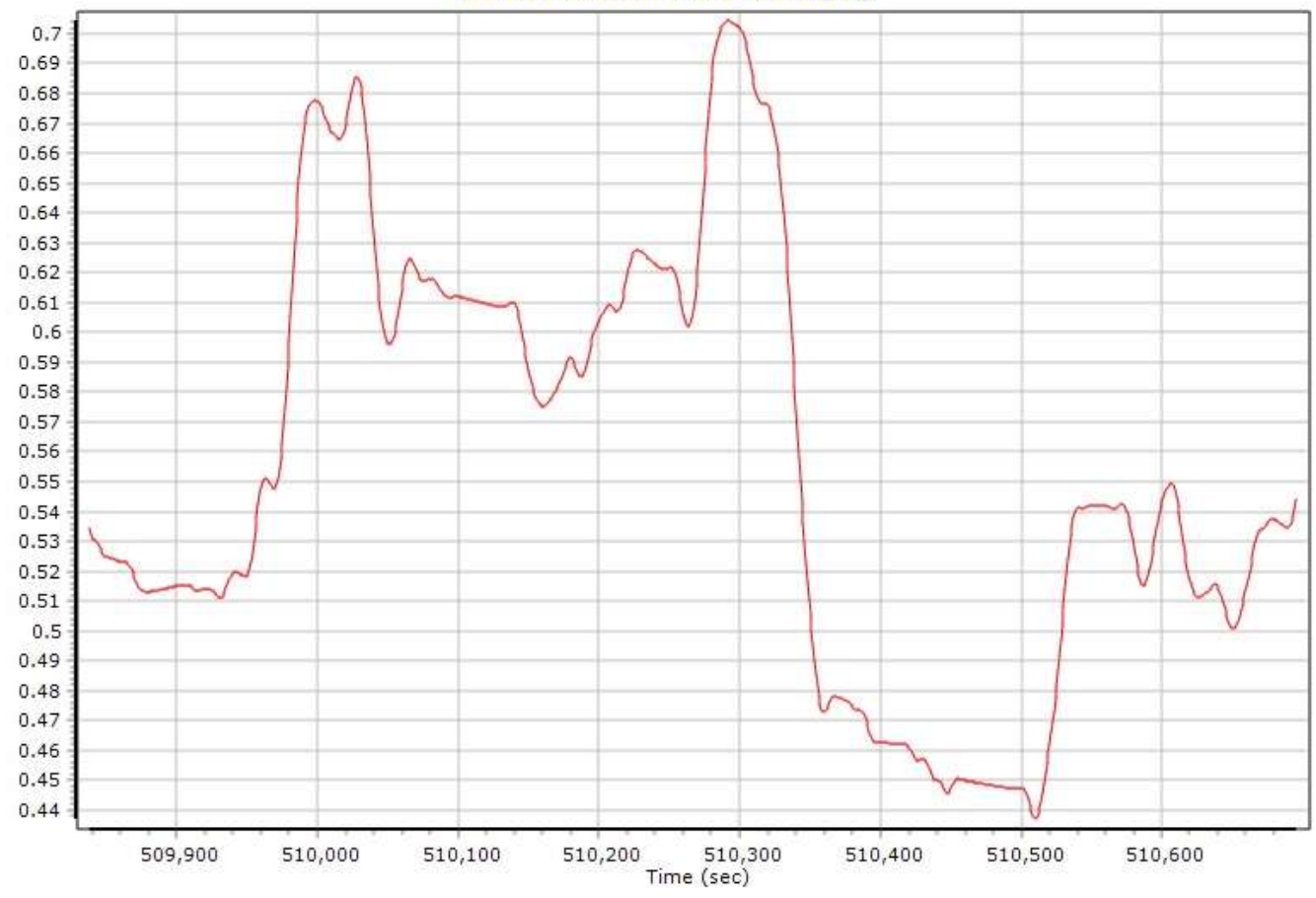

Fig 5.29: MMS SBET Down position RMS (m)

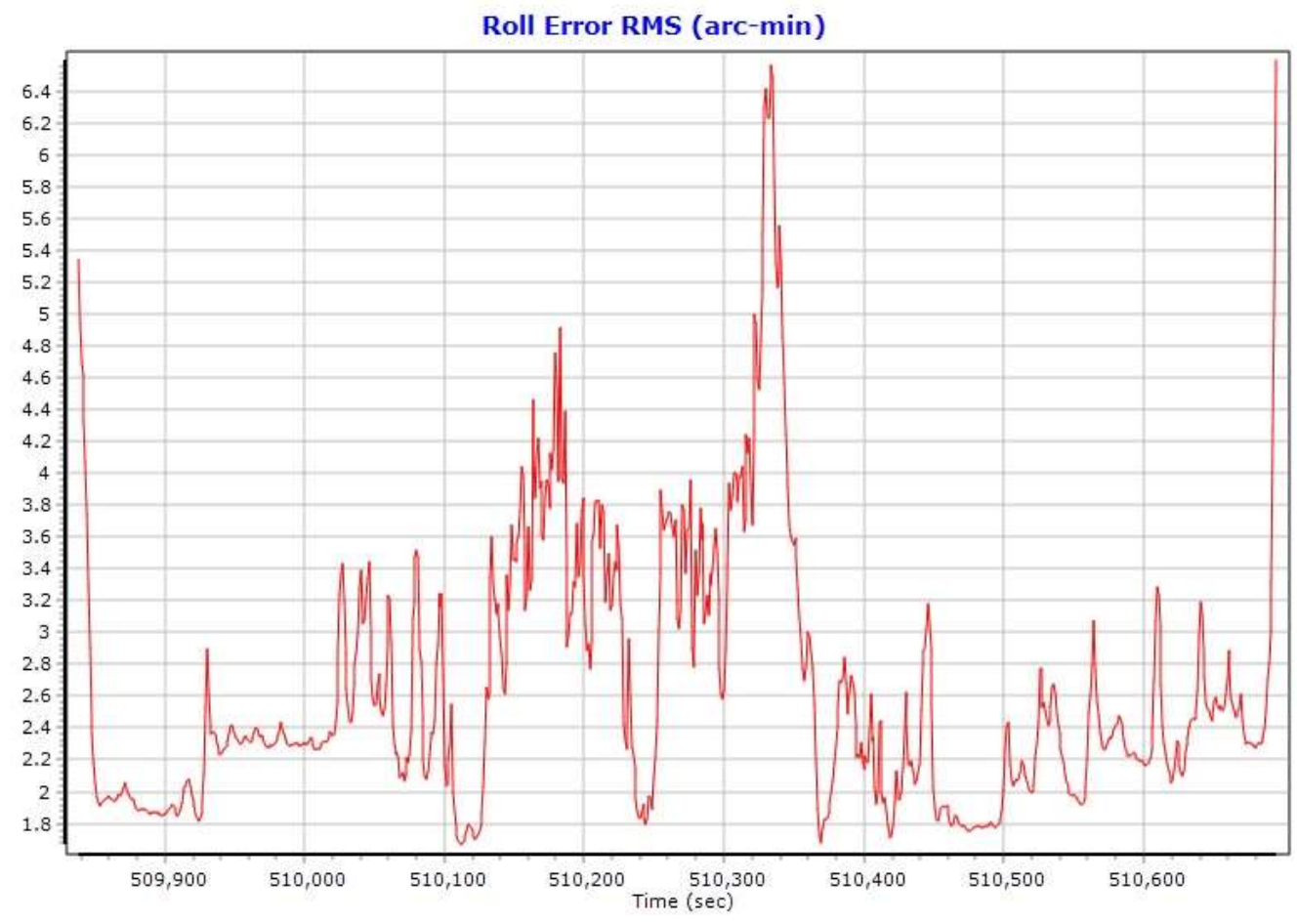

Fig 5.30: MMS SBET roll attitude RMS (arc-min) 
Pitch Error RMS (arc-min)

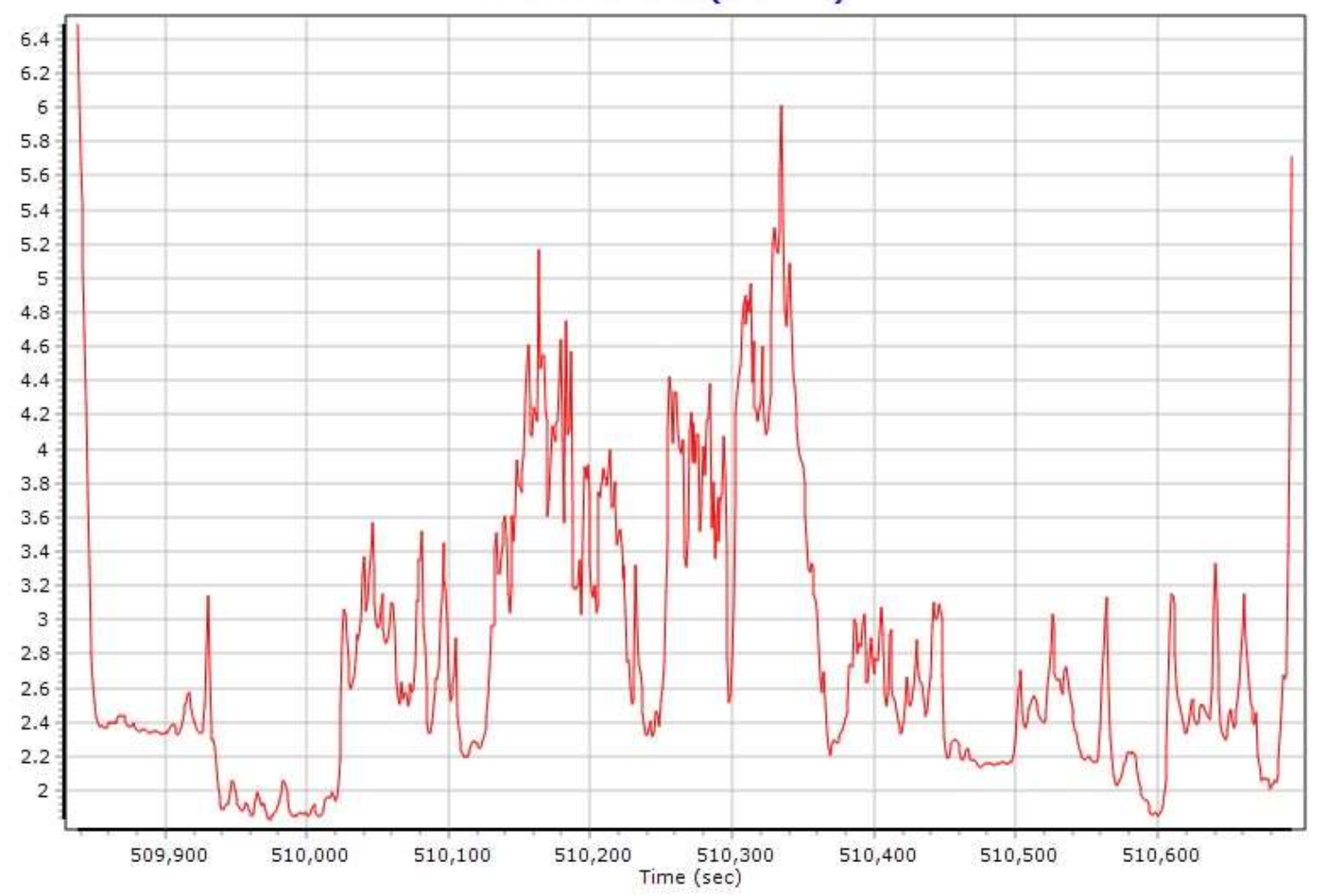

Fig 5.31: MMS SBET pitch attitude RMS (arc-min)

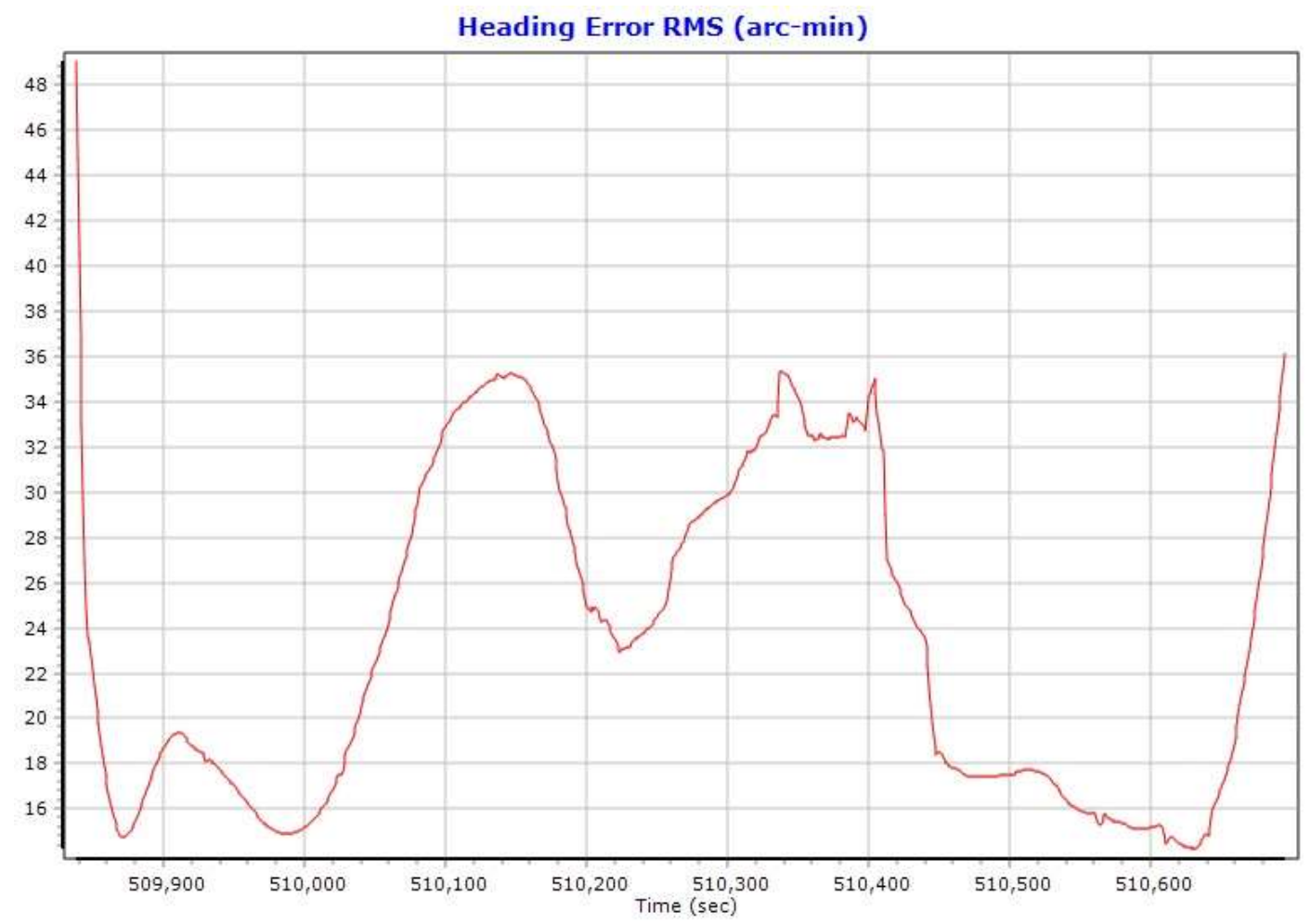

Fig 5.32: MMS SBET heading attitude RMS (arc-min) 
As anticipated and shown in Figs. 5.27 to 5.32, the least precise measurements occur in the down position and in the heading attitude determination. The north and east position RMS errors are 19 and $16 \mathrm{~cm}$, respectively, while the down position RMS error is $0.57 \mathrm{~cm}$. On the other hand, the attitude RMS errors are $0.04^{\circ}$ for the roll, $0.05^{\circ}$ for the pitch, and $0.4^{\circ}$ for the heading, which is one order of magnitude worse than the roll and pitch precision. Keep in mind that the base station was not deployed on a previously known control point, which means that the precision reported should be regarded a measure of the relative accuracy.

To better enhance the absolute geolocation accuracy, the base station recorded readings have been further processed through the NRCAN PPP service to provide decimeter-level absolute accuracy. The assessment of the LiDAR data point cloud accuracy is challenging due to the non-selective nature of the points captured. The challenge is further complicated if the assessment involves more than one data source. A few of the differences between the data mapped by the developed system and the accurate aerial LiDAR can be seen in the horizontal and vertical datum, the point spacing, and the coverage field of view.

The resultant point cloud of the mapped area using the developed system is superimposed on the accurate aerial LiDAR dataset along with the accurate orthophoto covering the area.

Comparing both of the data sources reveals that the aerial data misses the details of the building sides, especially buildings of a few stories, whereas the system developed mainly captures the building sides but lacks the top of the roofs. The orthophoto provides realistic true color imagery. The data from the aerial LiDAR mission is shown in Fig. 5.33. Besides the ability to check the accuracy of the mapped data by the fusion of the mapped data from the developed system and the LiDAR aerial coverage, superimposing the data mapped by the developed system at hand obviously fills in the missing building façades from the aerial data coverage, proving the benefits gained towards data completeness. 


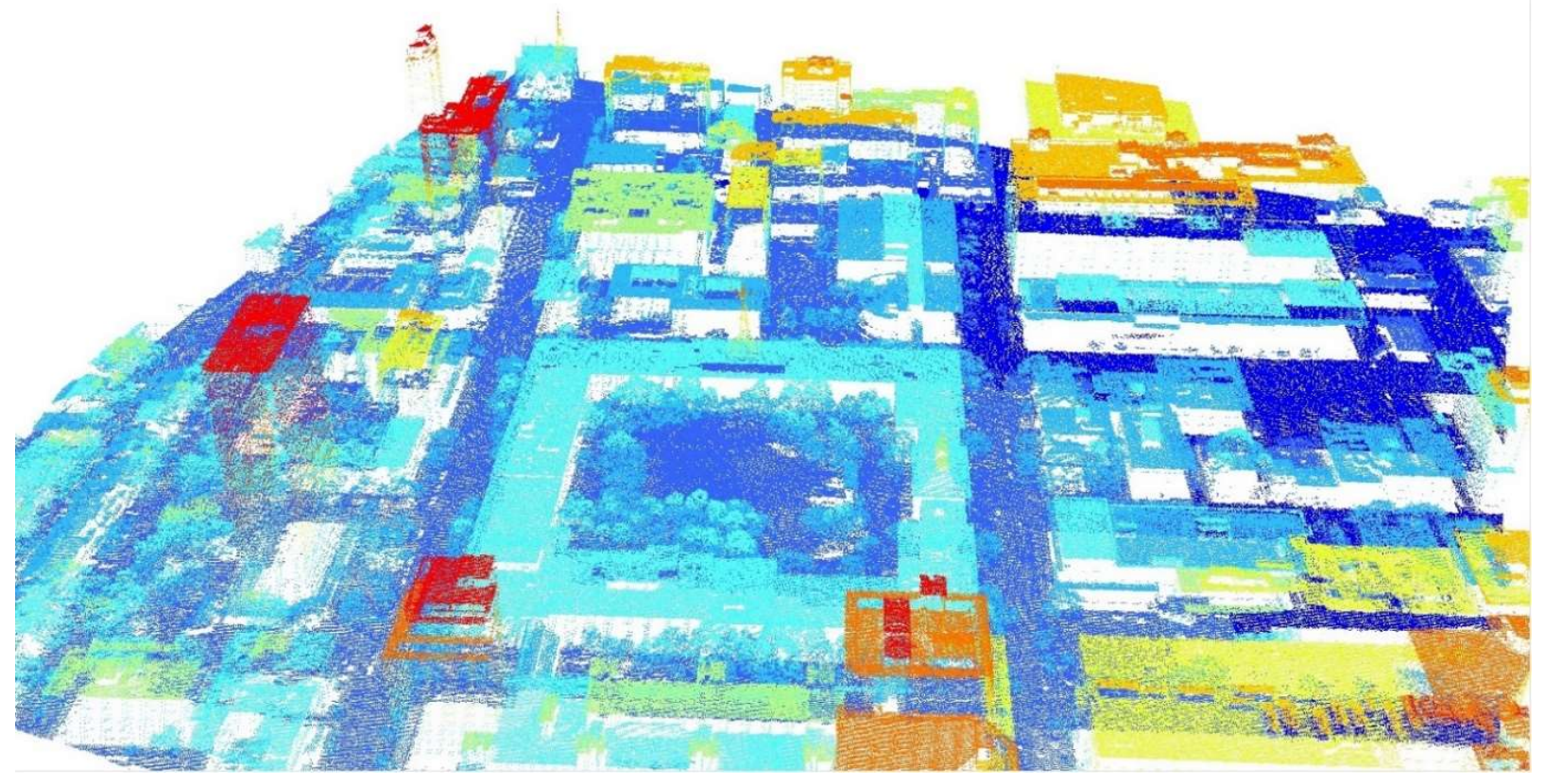

Fig 5.33: Aerial LiDAR data coverage (color coded by height)

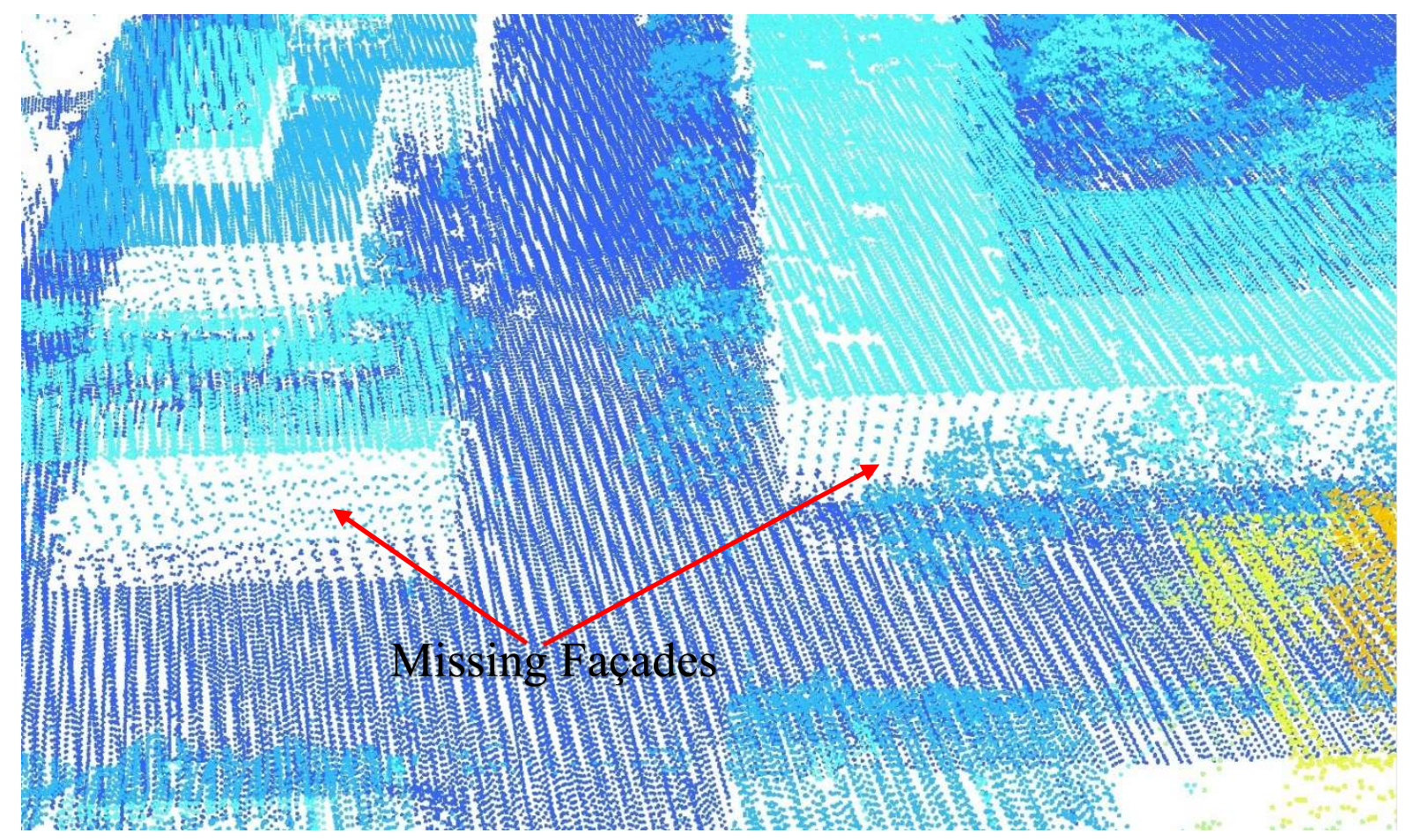

Fig 5.34: Aerial LiDAR data coverage (Missing areas);

Missing façades are white 


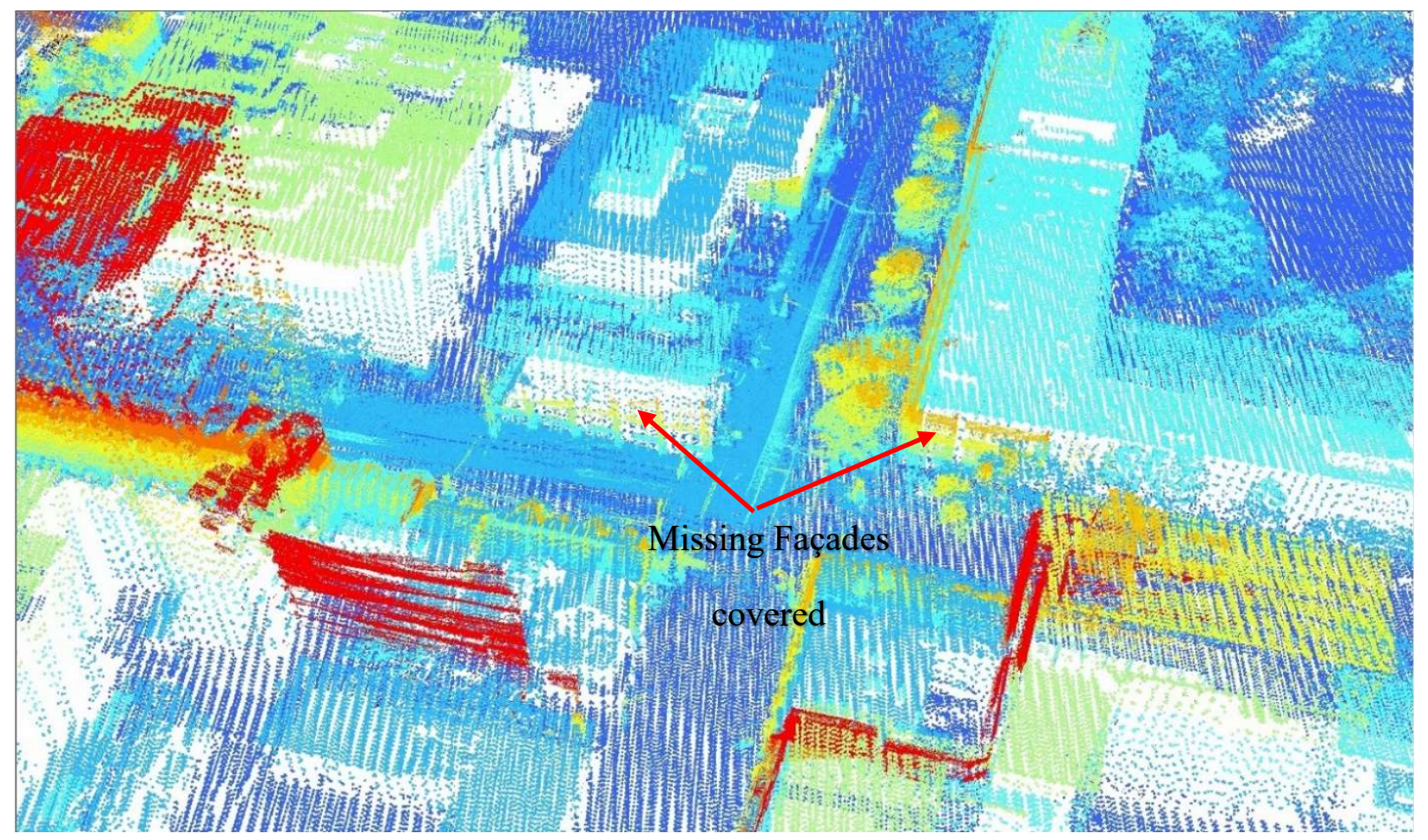

Fig 5.35: Data mapped by the developed system \& Aerial LiDAR data coverage (color coded by height); Missing façades are filled

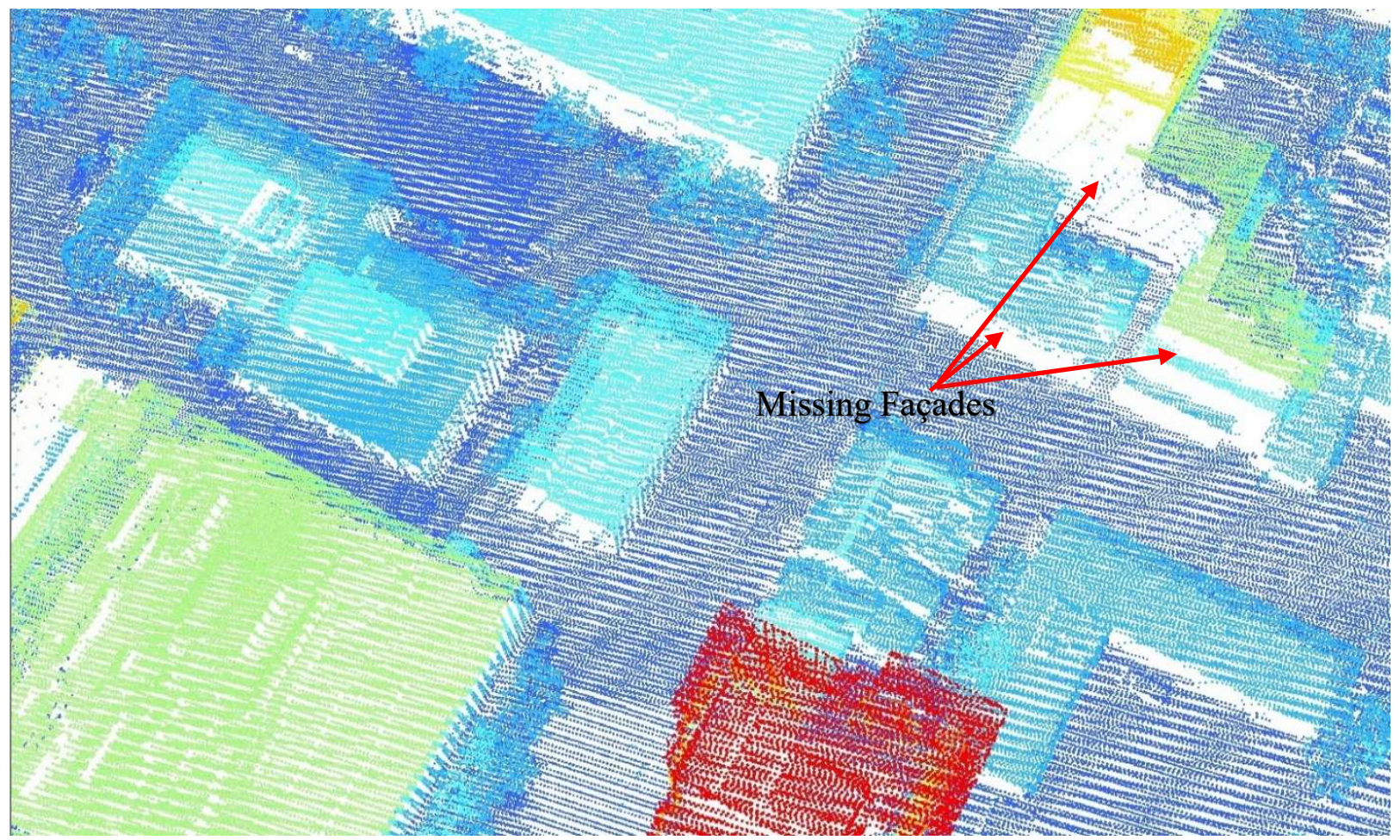

Fig 5.36: Aerial LiDAR data coverage 2 (color coded by height); Missing façades are white 


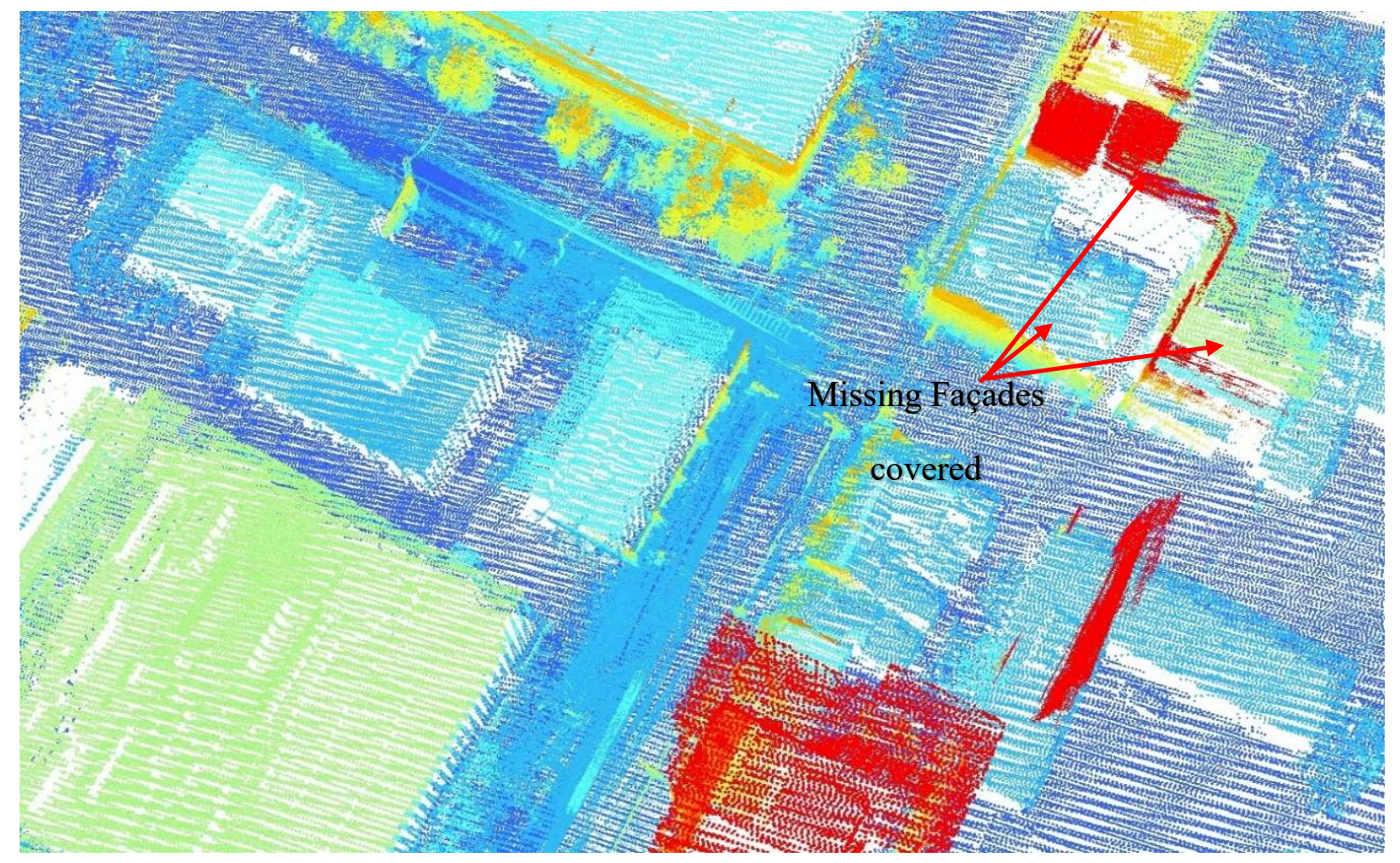

Fig 5.37: Aerial LiDAR data coverage 2 (color coded by height); Missing façades are filled

The qualitative assessment revealed a very good alignment between the two datasets, as highlighted in the four immediate preceding figures. An added advantage is achieved by the fusion of the two data sources to give coverage of both the façades and the roof tops. Moreover, the street furniture (e.g., traffic signs and light poles) is clearly mapped by the developed system while being obviously missed by the aerial coverage. Due to the difference in vertical datum associated with the two different datasets (the aerial LiDAR and the data mapped by the developed system), and in order to estimate the height offset between the two datasets, a ground plan representing road surfaces in both datasets has been extracted. Based on this, the vertical difference between the two planes in the up direction has been measured and applied to the data mapped using the developed system. Upon checking the aerial LiDAR data and the orthophoto, deviations in the order of $\sim 2 \mathrm{~m}$, especially at the roof edges of high-rise buildings, as illustrated in Fig. 5.38 was observed.

Concerning the assessment of the mapped area using the developed system, the aerial LiDAR data has been deemed the ground truth data and the orthophoto has been used for the better visual interpretation for both point clouds (the aerial LiDAR and the MMS from the developed system). In addition, the system repeatability and relative performance has been assessed by the utilization of two opposite data collection directions. The deviations between the same features mapped from the two opposite directions have been assessed as well, showing a very good alignment other than the calibration for the boresight angles and lever arm misalignments. A sample of the results of the assessment is shown in Figs. 5.39 to 5.43. . 


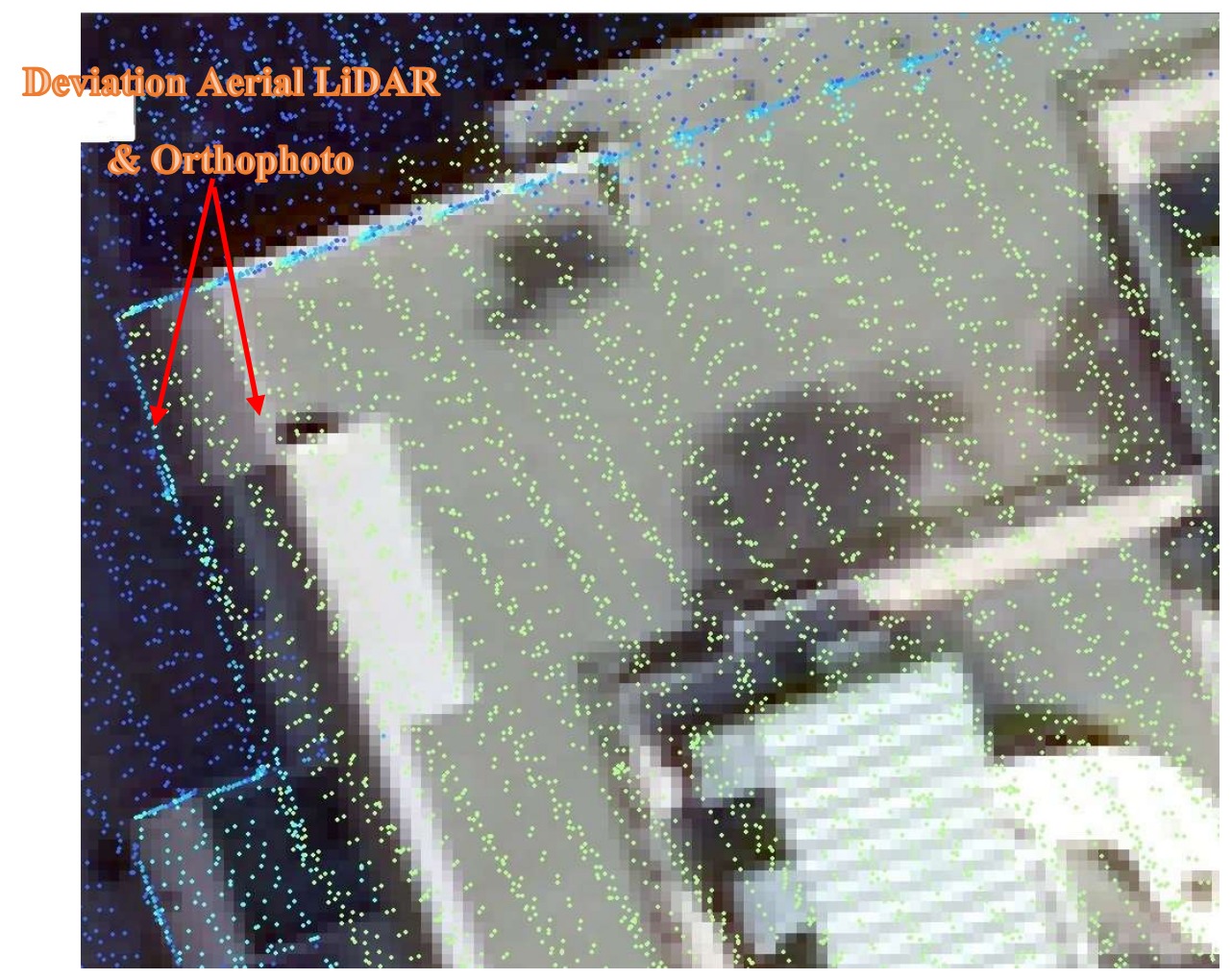

Fig 5.38: Aerial LiDAR data and the orthophoto deviations (same building side mapped by the orthophoto and the LiDAR aerial coverage)

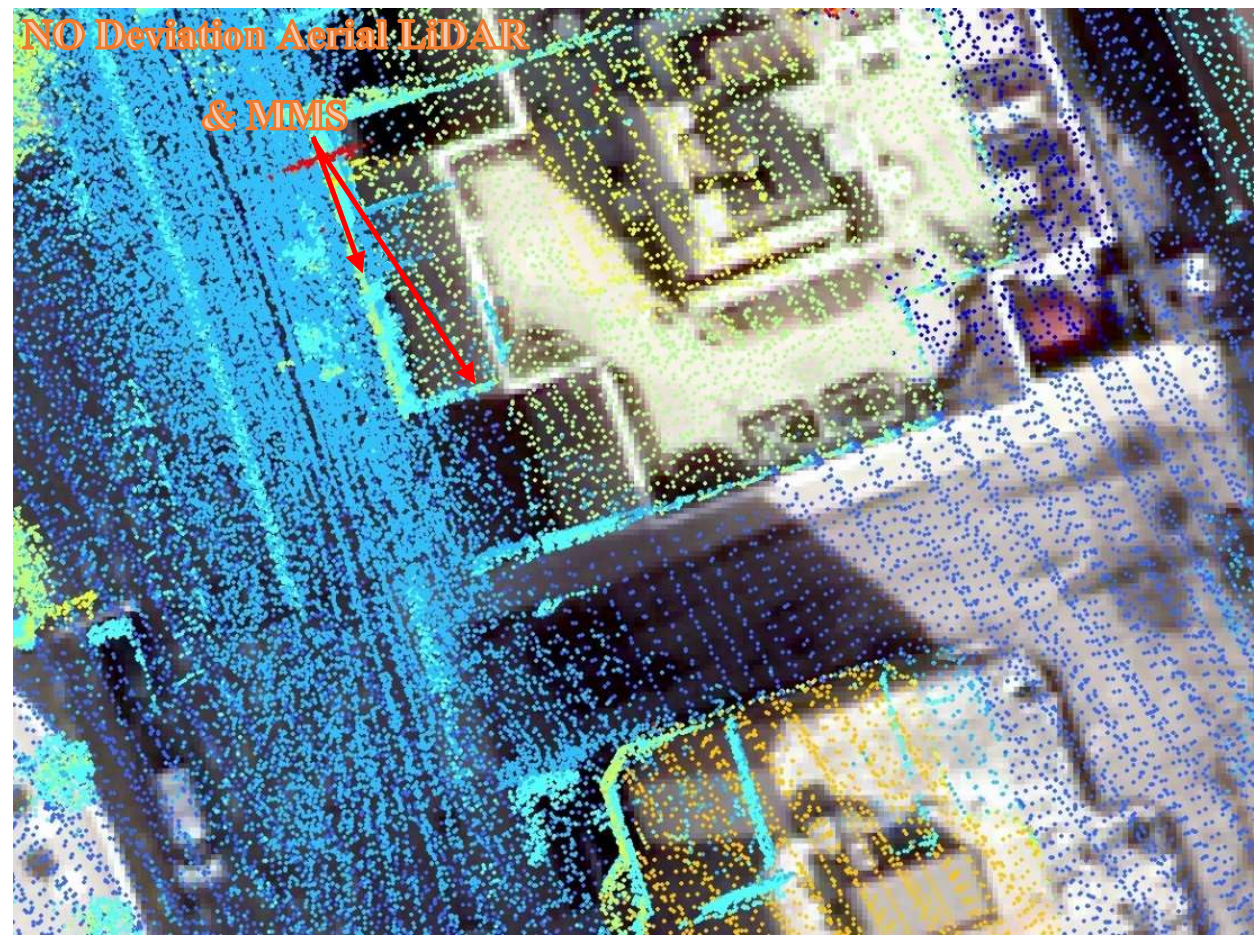

Fig 5.39: MMS data no deviation with Aerial LiDAR data; the orthophoto as background (MMS data shown in yellow and Aerial coverage shown in cyan) 


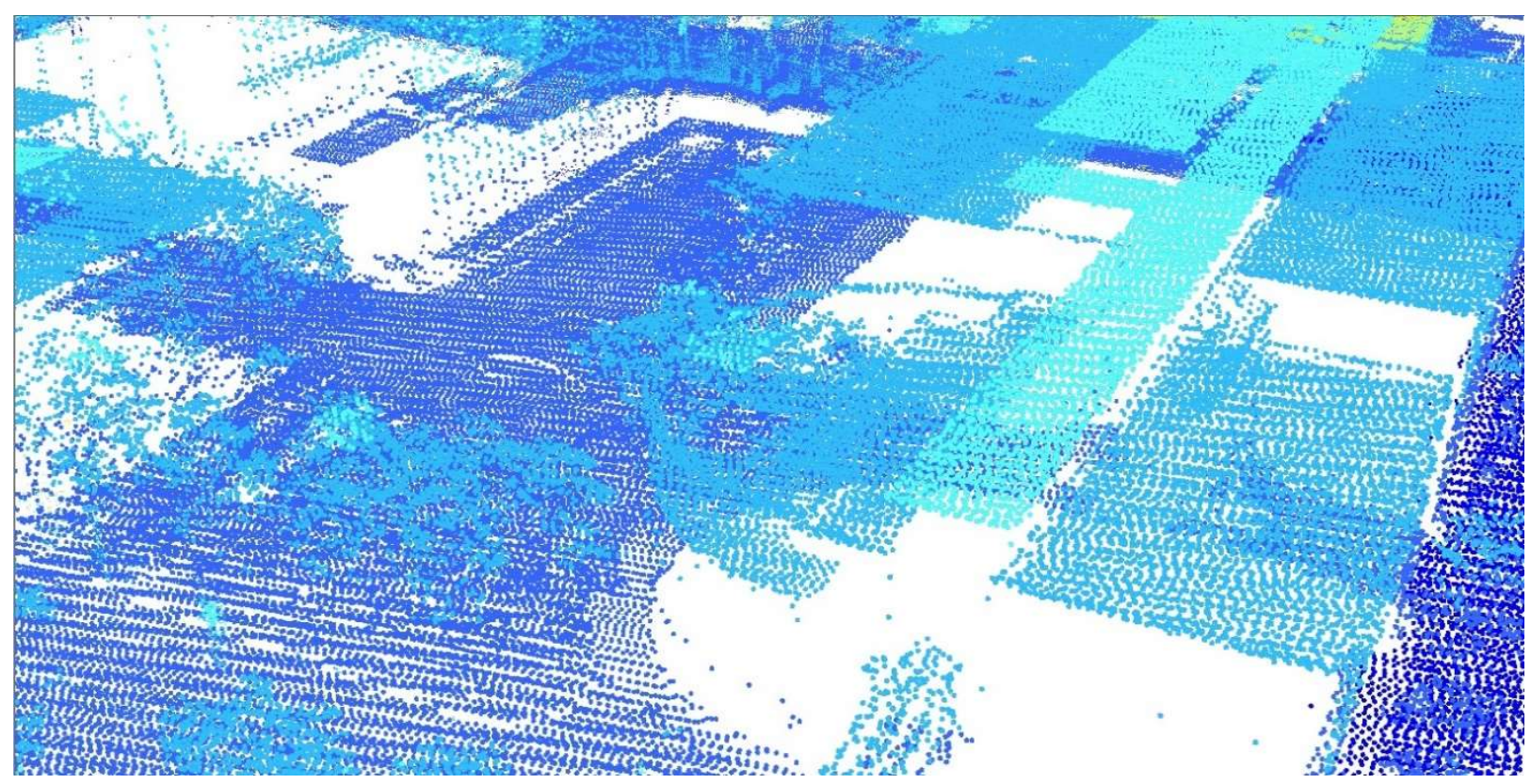

Fig 5.40: Area coverage (Aerial LiDAR only)

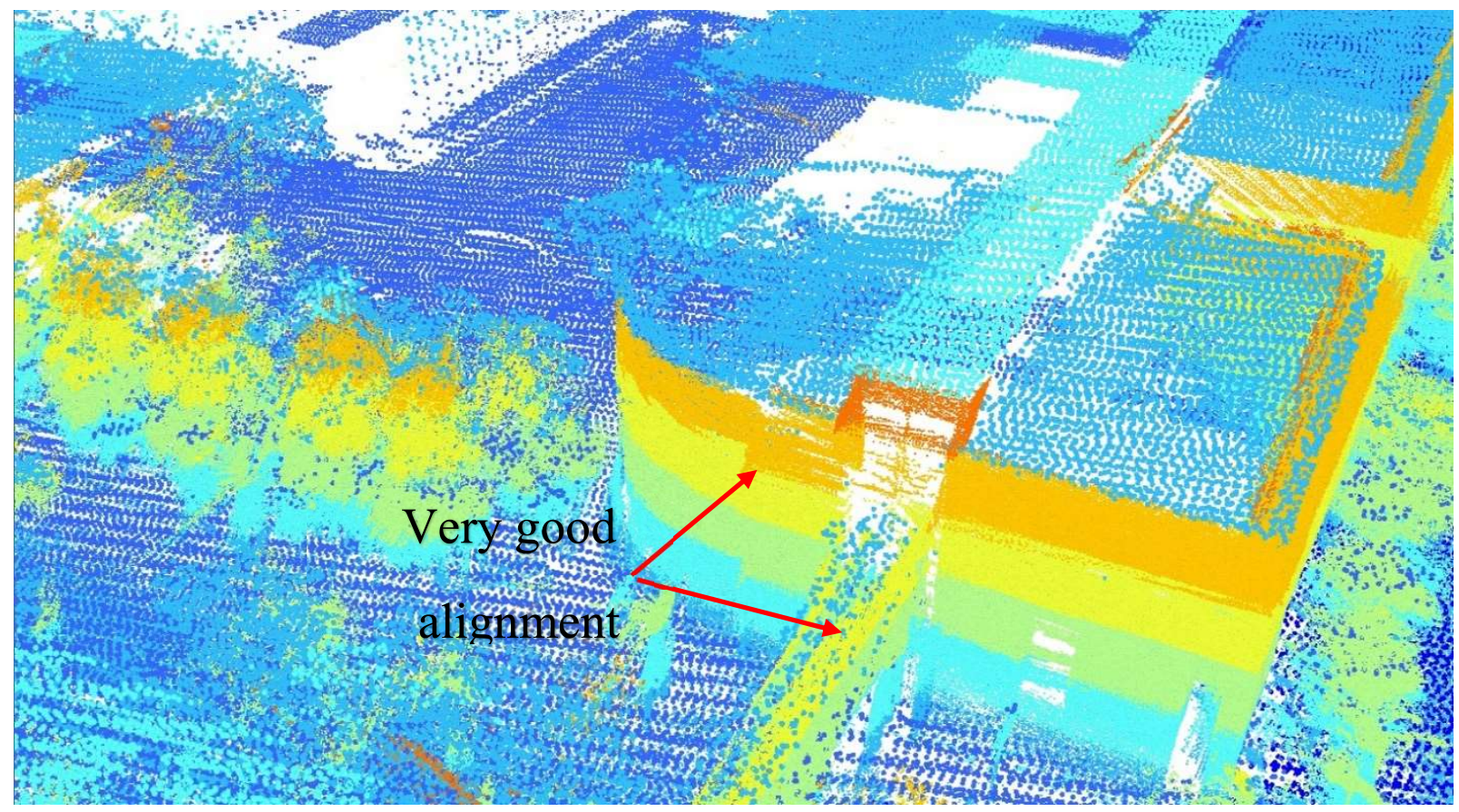

Fig 5.41: Area coverage (MMS and aerial LiDAR) 


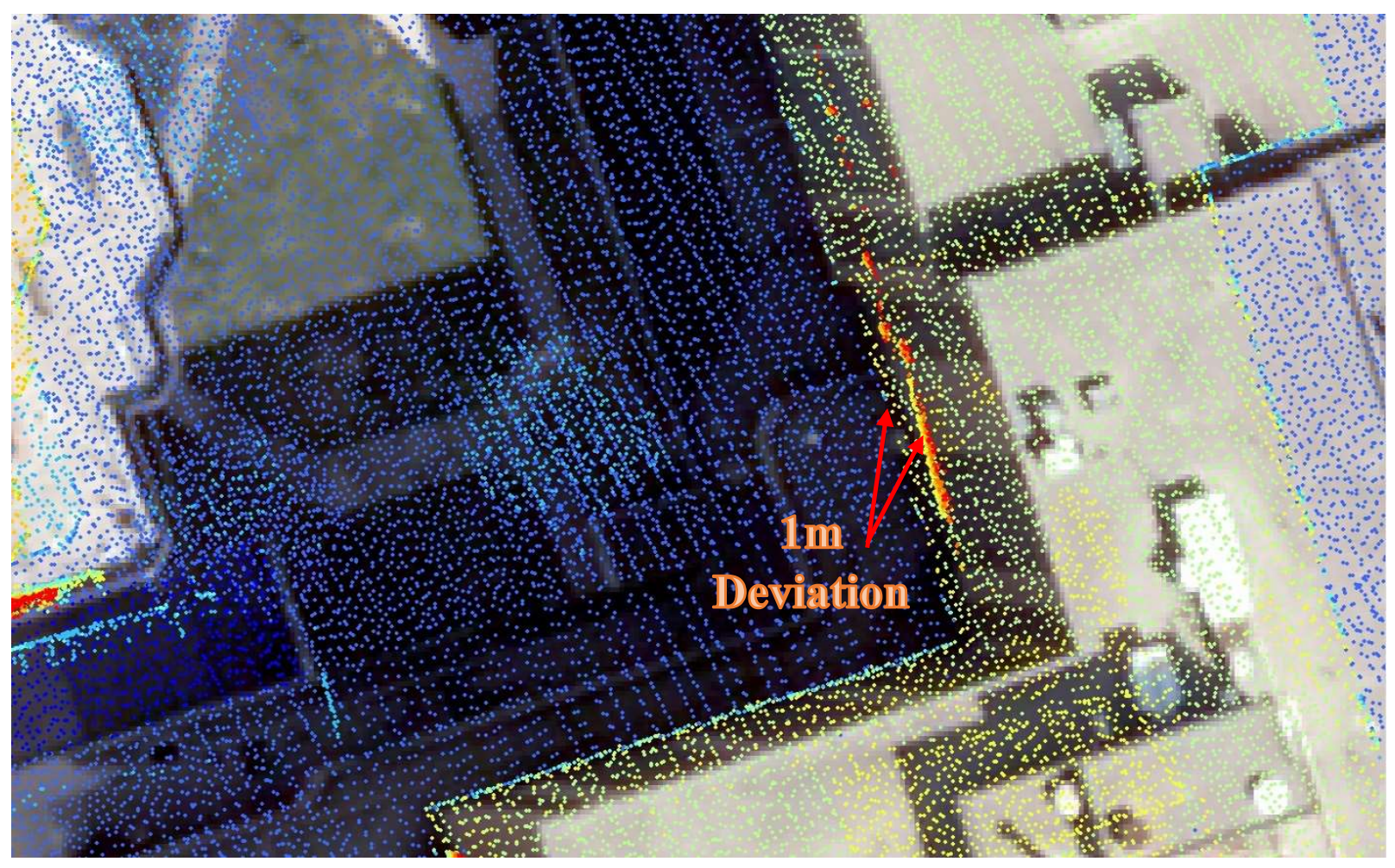

Fig 5.42: Deviation between MMS and aerial LiDAR

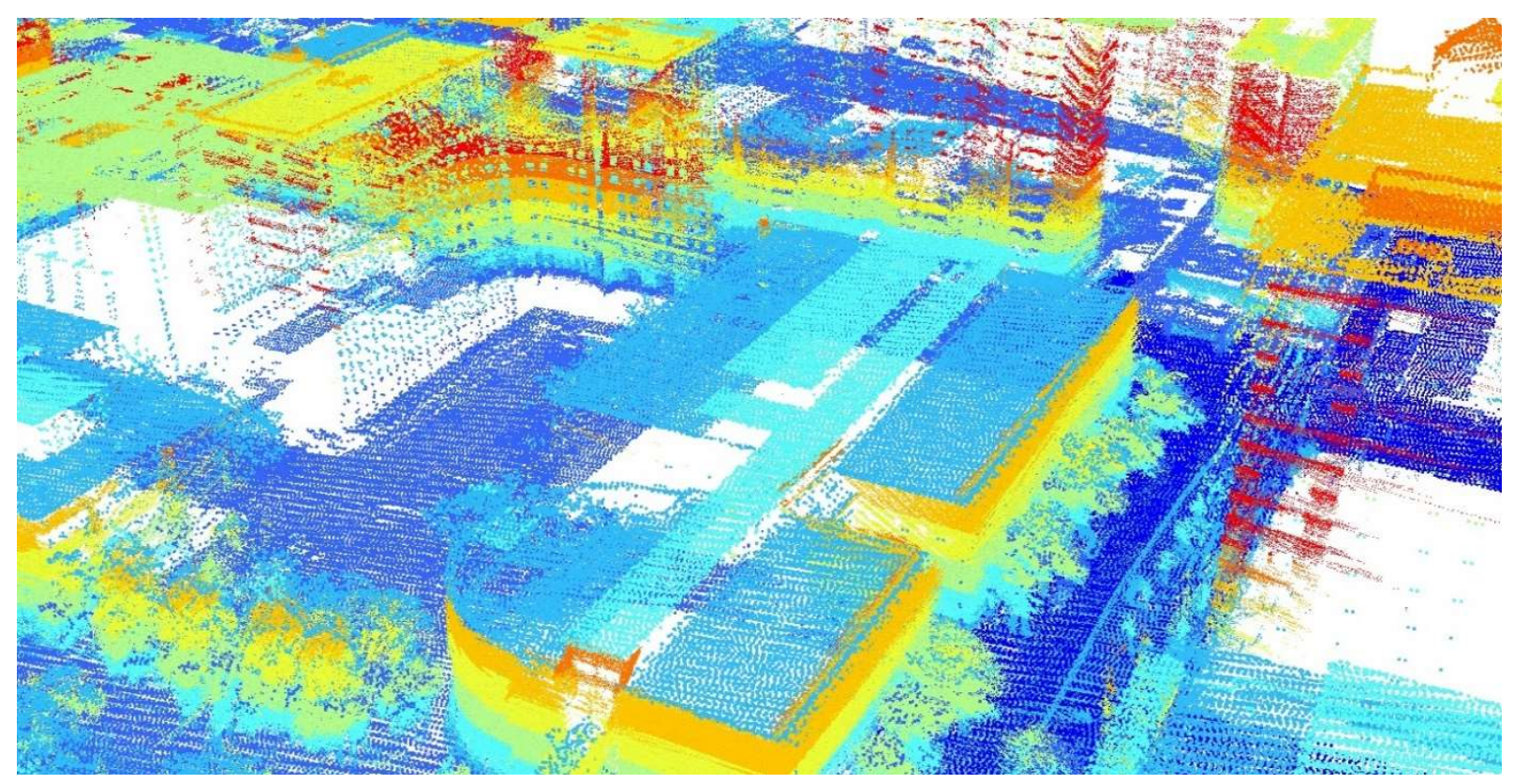

Fig 5.43: MMS \& aerial LiDAR complementary coverage 
Qualitatively, the deviation between the data of the area mapped with the developed system and the aerial LiDAR data has been assessed and found to align well in many areas as highlighted in Fig 5.41. On the other hand, the quantitative assessment of 3D point clouds is challenging. The developed uncertainty model provides a statistically-based model that can be used for the data validation for the $3 \mathrm{D}$ point gerorefrencing. The application of the uncertainty model provides the accuracy estimate covering the whole point cloud area. This is in contrast with using reference points. The accuracy estimated using reference points may not apply to the whole 3D point cloud coverage and it requires more resources that may not be readily available.

Using the precision values reported for the position and attitude for the mission trajectory and the precision values for the VLP-16 as described in section 4.4, the corresponding 3D georeferenced point cloud positional accuracy is $64 \mathrm{~cm}(1 \sigma$ at $25 \mathrm{~m})$. Moreover, by the aid of the orthophoto interpretation a number of measured distances between recognizable linear and planar primitives in both the MMS point cloud and the aerial LiDAR ground truth data has been measured. The deviation observed in many areas was in the decimeter level and the vertical deviation was less, but still some areas with a deviation of around sub meter $-2 \mathrm{~m}$ horizontal has also been observed as shown in Fig 5.42. This may be attributed to the different reference datum both horizontally and vertically for both MMS and the aerial LiDAR datasets, the base station has been on a non-known coordinates control point. In addition, the APX-15 is originally optimized to be used in UAVapplications with a clear open sky view for the GNSS signals, thus the multipath effect within the urban area mapped will degrade the performance. The vibration and shaking of the system while enclosed in the initial enclosure used. Figure 5.41 exhibits the very good alignment between the aerial LiDAR data and the MMS data collected, while in Fig. 5.43, the added benefit of the complementary coverage between both the MMS and the aerial coverage is clear.

The utilization of the new developed mapping system and its deployment as an MMS has been proven to be successful, as illustrated by both the qualitative assessment of the visual interpretation of the mapped area and the quantitative assessment with the validation against a survey-grade aerial LiDAR dataset.

Moreover, the combination of two different coverages (aerial and MMS) shows complementary data coverage with a higher percentage of data completion. This is another important factor that highlights the significance of the developed architecture, as the developed system is platformindependent and the same mapping system can readily be applied in MMS and ULS mode. 


\subsection{MMS Tilted Orientation}

The VLP-16 is a LiDAR sensor developed originally for autonomous vehicles navigation. The deployment of the sensor with its spinning axis almost vertical is deemed more optimal, as it allows for the use of the full range of the sensor to see objects far from the vehicle, enabling the autonomous control system to act accordingly. This orientation of deployment is considered suboptimal for the mapping purposes, since it will still allow for vertical gaps between the LiDAR beam readings. In addition, the relatively long range of scanning would map the features multiple times, as the vehicle pass-by, which magnifies the effect of any imprecision in the DG mapping process

In order to test the benefits gained in the mapping process and the effect on the mapped data, a tilted orientation of the system is deployed in the MMS mode. The inclination of the system such that the VLP-16 spinning axis is nearly vertical will flip the $360^{\circ}$ field of view for the sensor across track to scan the buildings and other features on both sides of the street, which is typically the main focus of any MMS application. Using the same approach, the degradation caused by having coarse gaps between the beams in the horizontal direction of the mapped objects (which may result in missing thin objects) can be compensated for as the vehicle naturally moves along the track direction.

The setup of the system with the applied orientation mounted using the practical efficient threearm suction cups mount is depicted in Fig 5.44.

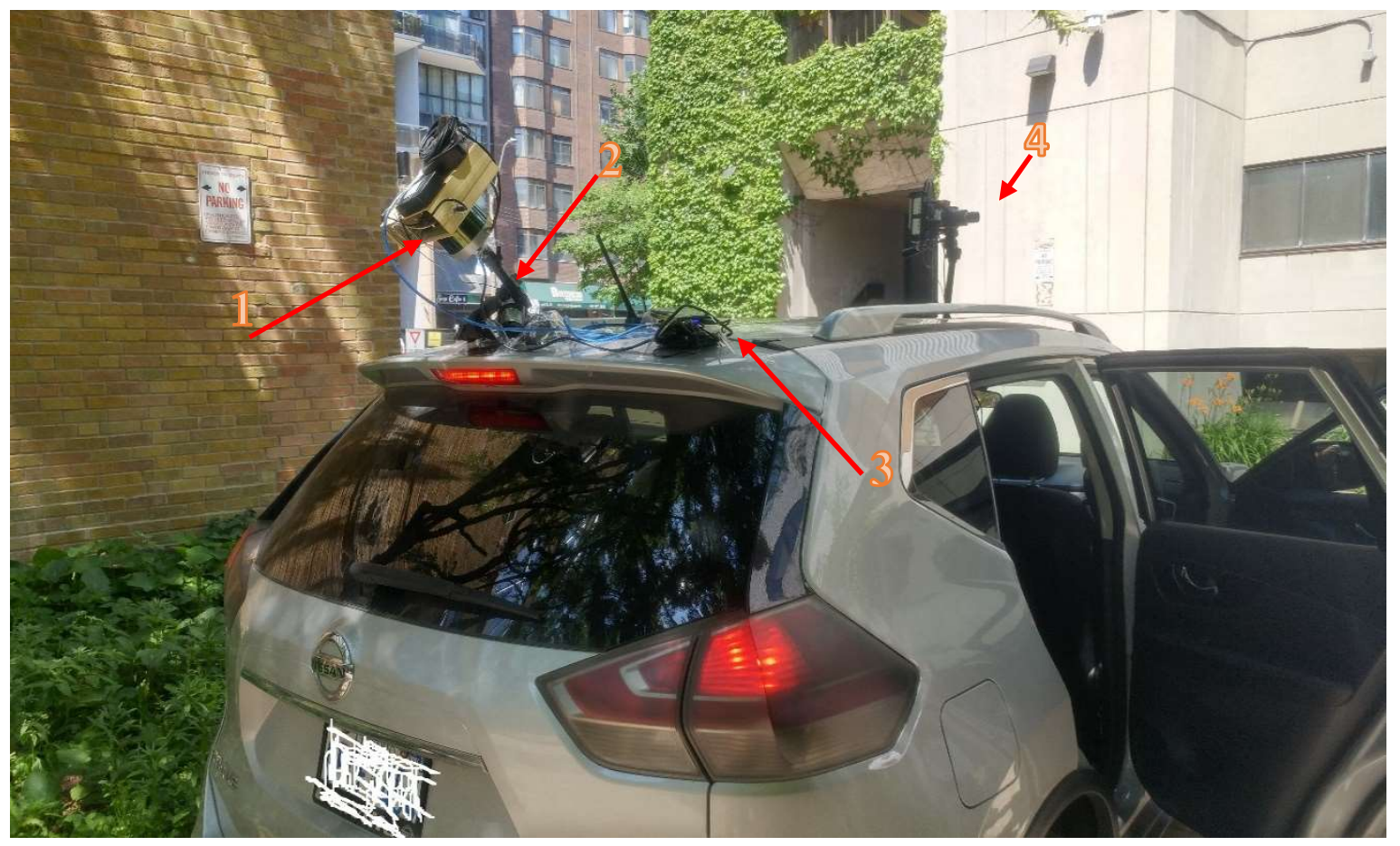

Fig 5.44: The system deployed in MMS mode

1) The system , 2) The car mount, 3) The control unit, 4) Hyperspectral camera 
Figure 5.44 shows the orientation applied to the system while mounted in MMS mode of operation. The control unit is decoupled from the system and placed apart to lessen the interference it may cause to the light small GNSS antenna used. Furthermore, the control unit allows for the full control and operation of the system without the need to use a laptop or any other device. The interfacing with the control unit is achieved through a 3.5-inch screen. This adds to the ease of deployment and practicality of the system usage. In addition, Fig. 5.44 show a hyperspectral camera whose data would be integrated with the mapping system in a future phase.

The system's multimodal sensor data integration follows the scheme described in Chapter 3, after which the direct georeferencing procedure (as detailed in Chapter 4) is applied. The original horizontal orientation sample reading is portrayed in Fig. 5.45, while the reading according to the orientation at hand applied is illustrated in Fig. 5.46. In both of these figures, we can see that differences between the horizontal and vertical fields of view in the two orientations are obvious.

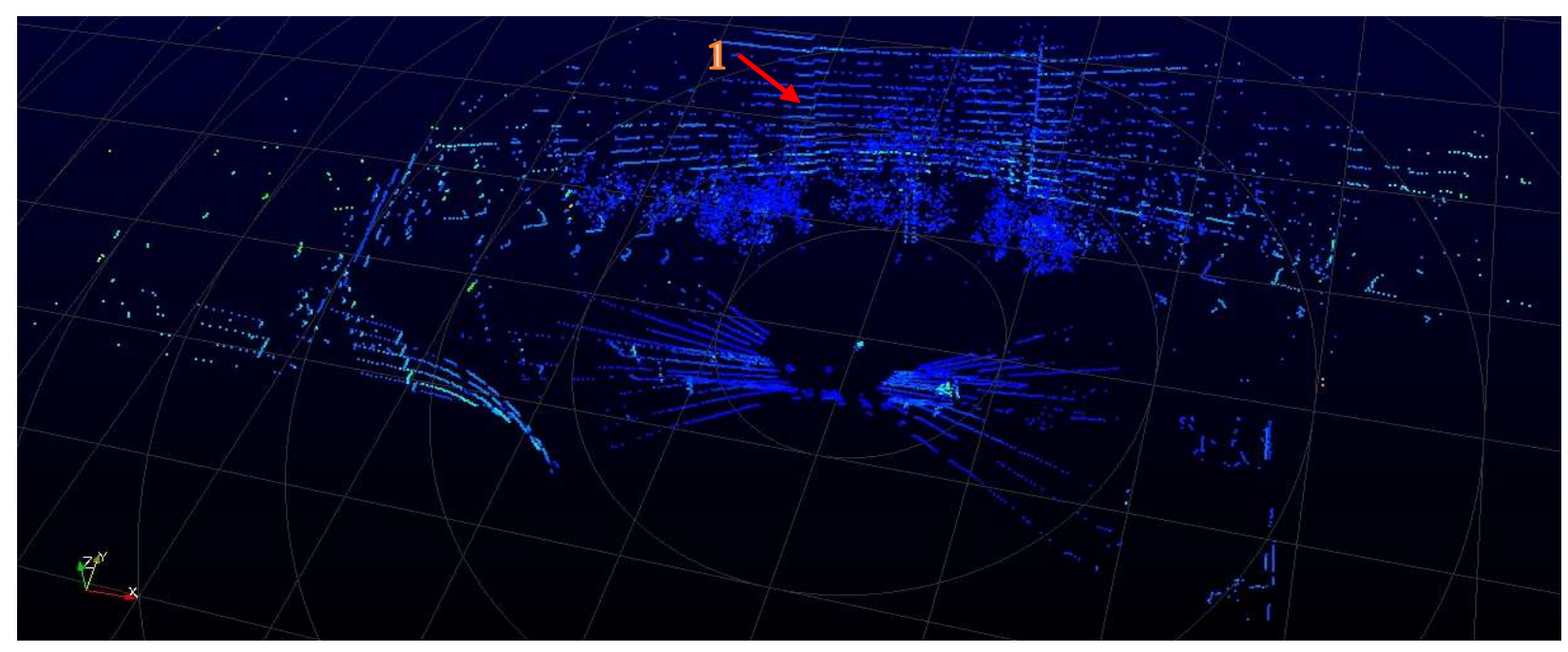

Fig 5.45: System sample reading (system horizontally mounted)

1) Vertical gaps

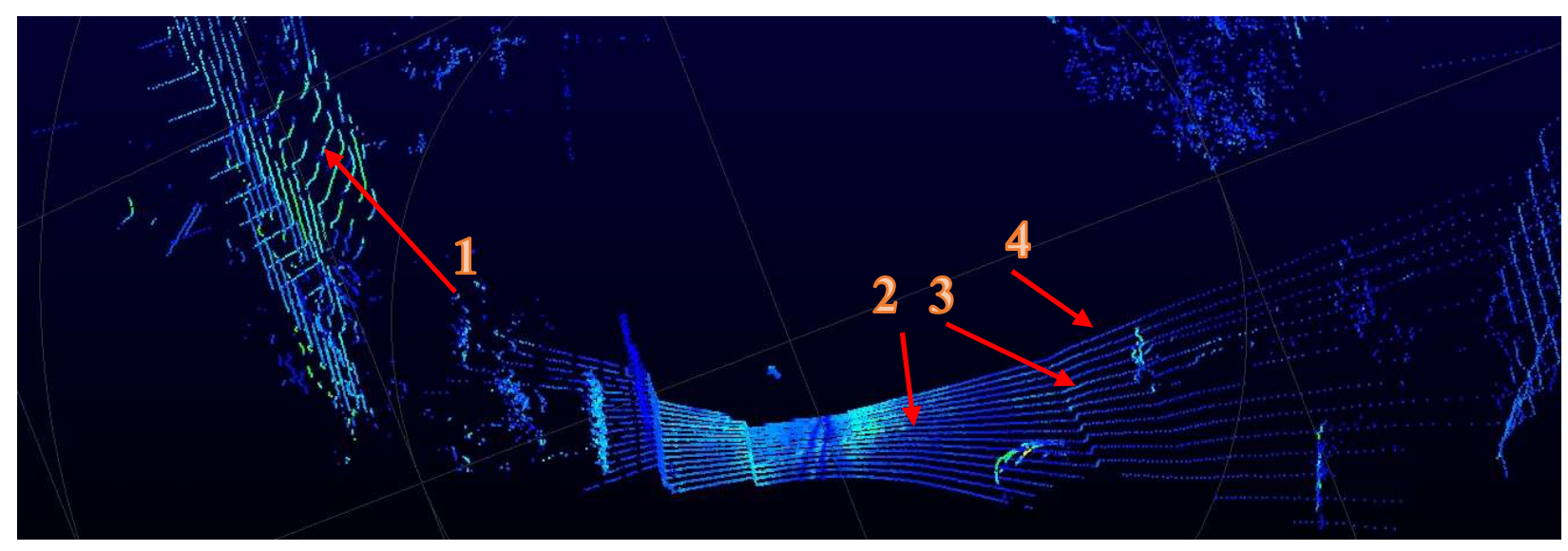

Fig 5.46: System sample reading (system mounted tilted)

1) Better mapping for higher objects 2) street floor 3) fine details ,curb, 4) horizontal gaps 
Figure 5.45 shows a longer range in the along-the-track direction, meaning that the same area will be mapped multiple times as the vehicle moves. This introduces cluttering in the resultant point cloud, as the imprecision in the attitudes and position will be emphasized with the multiple scanning of the same features while moving. Moreover, it misses mapping the street ground. On the other hand, as indicated in Fig. 5.46, the frame-reading occurs when the system is oriented and the street level floor is correctly mapped. The view in the across-track direction is considerably shrunken compared to the horizontal orientation of the system, thus decreasing the cluttering associated with mapping the features multiple times. In addition, fine details such as the street curb are mapped, with higher features being better mapped.

It is worth noting that the vertical gaps experienced with the horizontal orientation shown in Fig. 5.45 are now flipped to horizontal gaps that are naturally filled by the car movement itself. This is further illustrated in Fig. 5.47. The Fig portray a building with a very clear representation, a traffic sign, a lamp post; even the intersection markings and hanging wires are correctly mapped as shown in Fig 5.48.

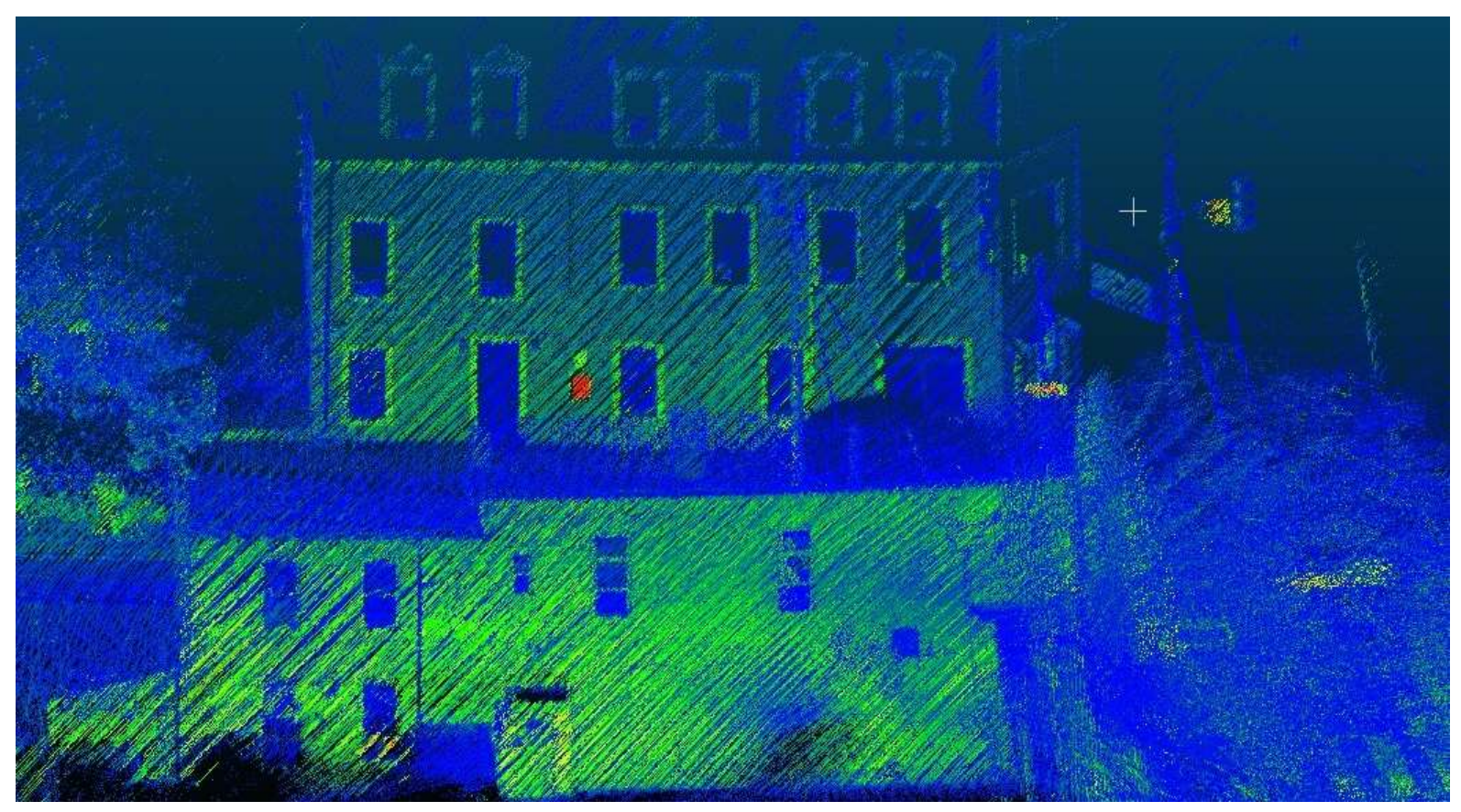

Fig 5.47: A sample Mapped area with tilted system mount 


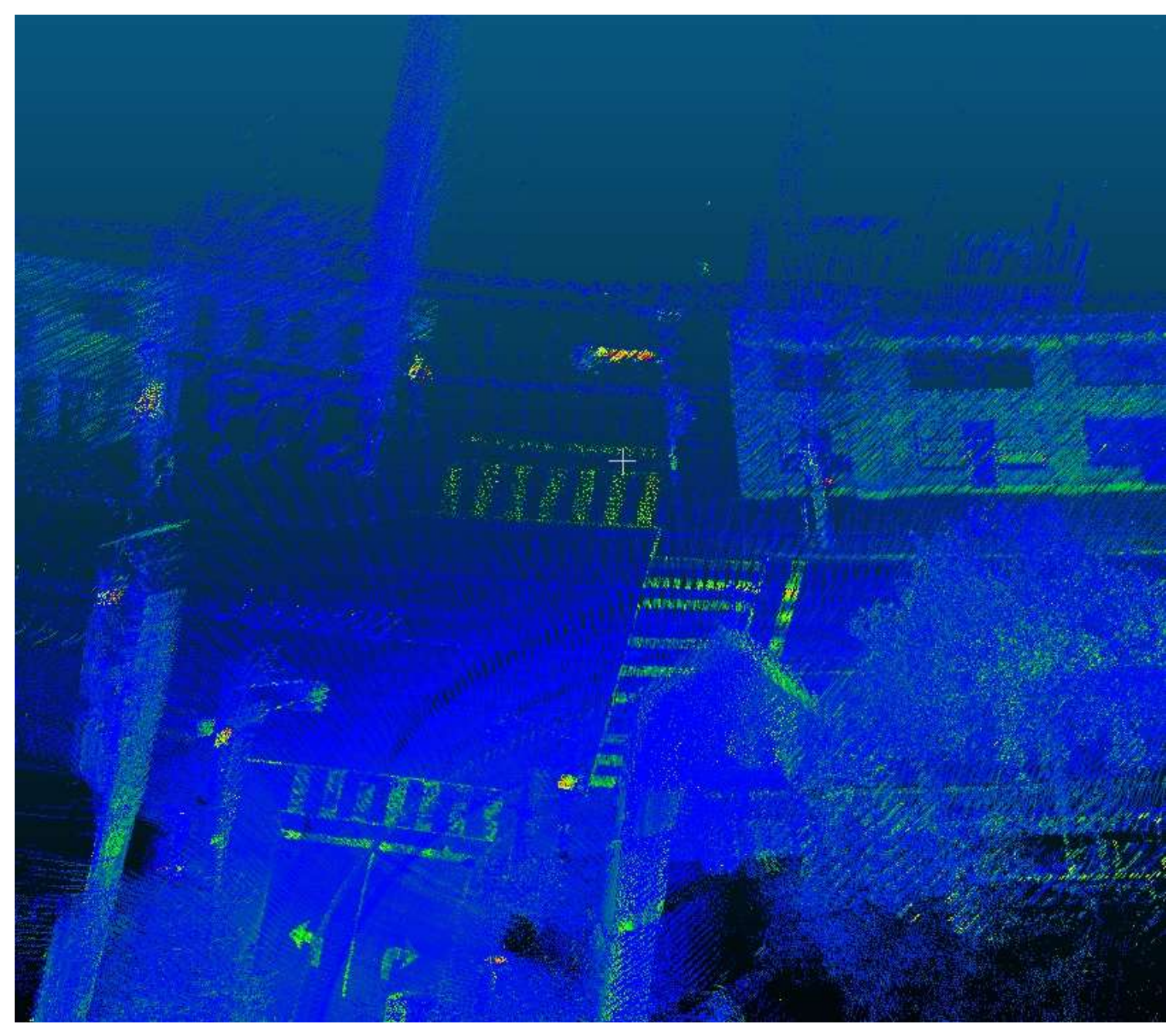

Fig 5.48: Sample of road markings mapped

In order to have a better natural color representation, a new 3D point cloud colorization approach is proposed and implemented. Several areas already have orthophoto imagery, and this imagery can be utilized in the LiDAR data colorization. The proposed and implemented procedure can be summarized as follows. To register the 3D LiDAR point cloud to the $2 \mathrm{D}$ imagery, the LiDAR data $3 \mathrm{D}$ point cloud is transformed to $2 \mathrm{D}$ imagery using the intensity values, following which the 2D$2 \mathrm{D}$ image registration can be performed. The 2D-2D image registration is carried out using a semiautomated process, where a high-pass filter is applied to the images to highlight edges and corners that can then be used as candidates for the control points on both images to perform the registration. Once the edges are highlighted, a number of interactively selected points on both imagery are used in the polynomial 2D-2D image registration equations.

A more challenging environment has also been tested, the available 2D imagery from Google Earth or Google Street View can be used in the colorization process, subject to receiving the proper copyrights permissions. However, due to the lack of geolocation information associated with the imagery, the different perspectives used to capture the images (in the case of Google Earth's aerial imagery), and to aid in the registration process, the point of view used to extract the images has 
been changed to resemble that of the LiDAR data MMS data collection. A number of interactively selected points (typically at rood intersections) has been used to register the LiDAR-based transformed 2D images and the Google Earth imagery

Once registered, the color information from the true color imagery is linked to the $3 \mathrm{D}$ point cloud and used in the colorization.

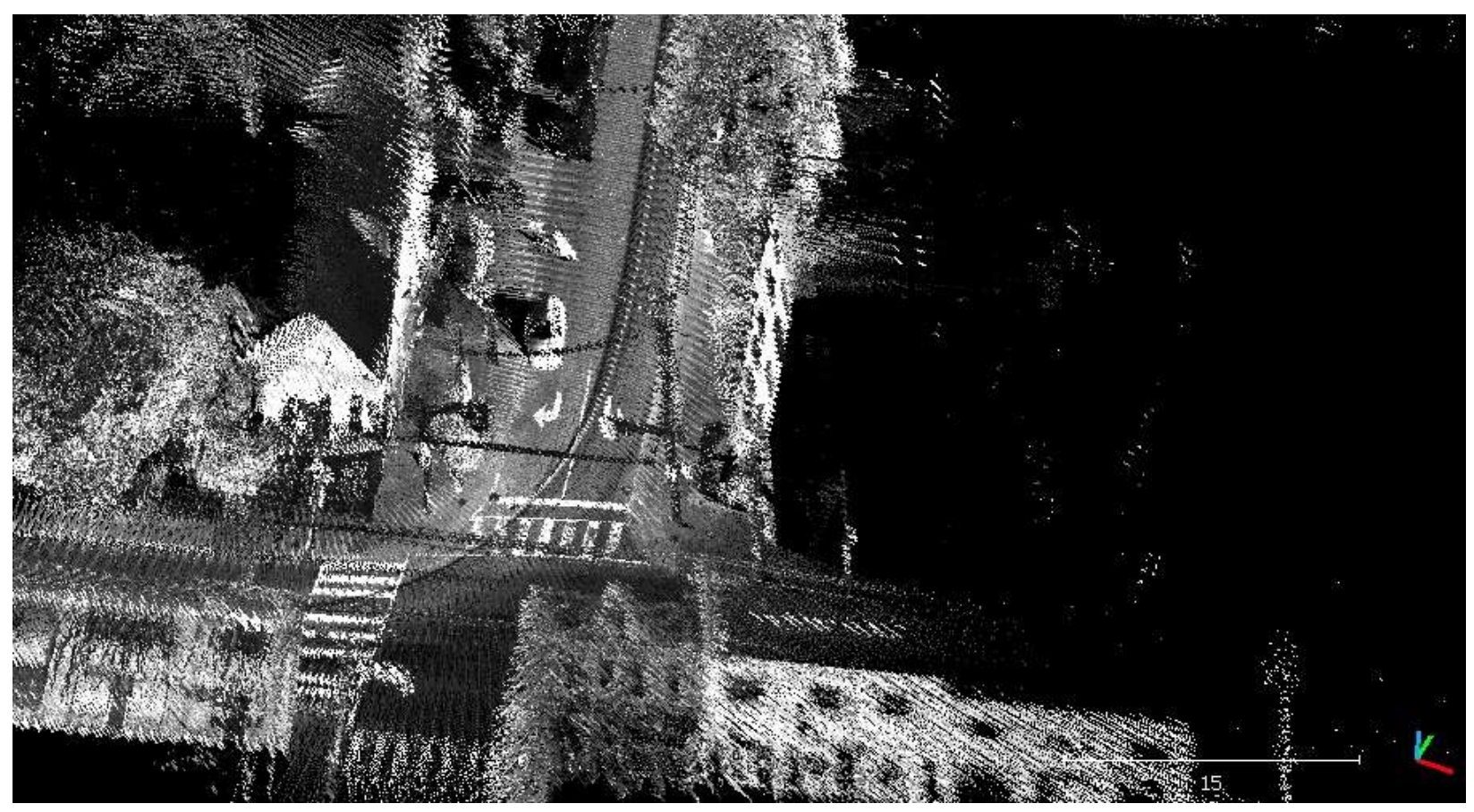

Fig 5.49: System Original point cloud 


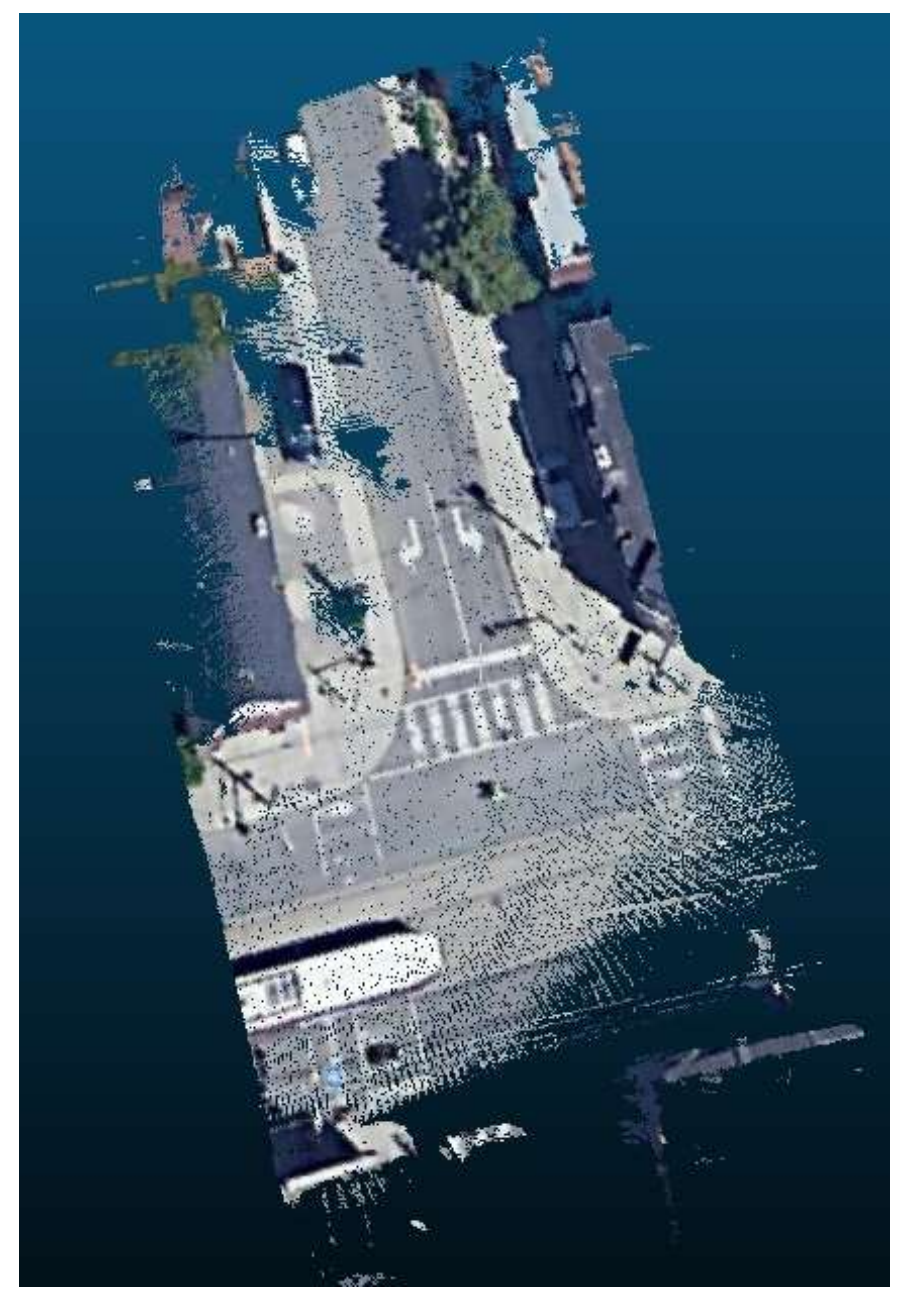

Fig 5.50: System colorized Point cloud

The original 3D point cloud and the colorized 3D point cloud after applying the colorization procedure are shown in Figs. 5.49 and 5.50, respectively. A comparison of these figures clearly shows the better visualization appeal when the point cloud is colorized. This improvement enhances the visual interpretation related-tasks, thus satisfying more end-user requirements. 


\section{THE MAPPING SYSTEM REALIZATION: UAV MODE OF OPERATION, SFM AND DSM GENERATION}

The final mode of operation to be tested is the deployment of the developed system in UAV-based mode. The system should meet the most stringent requirements to be deployed in UAV LiDAR mapping system ULS mode, as the ULS mode poses the strictest SWaP system characteristics that need to be met. This is due to the UAVs limited payload, short flight endurance, and the vibration and interference from the UAV own electronics. In addition, the limited space on the UAV adds another challenge to the ULS deployment.

For the number of advantages, the multirotor UAV feature, as ease of use, accessibility, operability in confined spaces, and lack of need for specific conditions at the field environment for take-off and landing (as opposed to the fixed-wing UAV), the multirotor type has been selected for the UAV deployment. Nevertheless, it worth highlighting that the developed system can also be deployed on fixed wings UAV. The DJI M600 pro is selected to be the UAV platform for deployment. The DJI M600 pro list of specifications is presented in Table 6.1 while its performance is presented in Table 6.2. The M600 is a professional drone that can easily accommodate the system developed and provide a descent time of flight.

Table 6.1: The DJI M600 pro UAV specs

\begin{tabular}{|l|l|}
\hline \multicolumn{2}{|c|}{ Attribute } \\
\hline Diagonal Wheelbase & $1133 \mathrm{~mm}$ \\
\hline Dimensions & $\begin{array}{l}1668 \mathrm{~mm} \times 1518 \mathrm{~mm} \times 727 \mathrm{~mm} \text { with propellers, } \\
\text { frame arms and GPS mount unfolded (including } \\
\text { landing gear) }\end{array}$ \\
\hline Weight (with six TB48S batteries) & $10 \mathrm{~kg}$ \\
\hline Max Takeoff Weight Recommended & $15.5 \mathrm{~kg}$ \\
\hline
\end{tabular}

Table 6.2: The DJI M600 pro Performance

\begin{tabular}{|l|l|}
\hline \multicolumn{2}{|c|}{ Attribute } \\
\hline Hovering Accuracy (P-GPS) & Vertical: $\pm 0.5 \mathrm{~m}$, Horizontal: $\pm 1.5 \mathrm{~m}$ \\
\hline Max Angular Velocity & Pitch: $300^{\circ} / \mathrm{s}$, Yaw: $150^{\circ} / \mathrm{s}$ \\
\hline
\end{tabular}




\begin{tabular}{|l|l|}
\hline Max Pitch Angle & $25^{\circ}$ \\
\hline Max Wind Resistance & $8 \mathrm{~m} / \mathrm{s}$ \\
\hline $\begin{array}{l}\text { Max Service Ceiling Above Sea } \\
\text { Level }\end{array}$ & $\begin{array}{l}2170 \mathrm{R} \text { propellers: } 2500 \mathrm{~m} \\
2195 \text { propellers: } 4500 \mathrm{~m}\end{array}$ \\
\hline Max Speed & $40 \mathrm{mph} / 65 \mathrm{kph}$ (no wind) \\
\hline $\begin{array}{l}\text { Hovering Time (with six TB48S } \\
\text { batteries) }\end{array}$ & No payload: $38 \mathrm{~min}, 5.5 \mathrm{~kg}$ payload: $18 \mathrm{~min}$ \\
\hline
\end{tabular}

Although the weight of the developed system is just $45 \%$ of the max payload for the DJI M600 pro UAV, the VLP-16 sensor used in the developed system can easily be replaced by the newer lighter-weight VLP-16 Lite, which saves the difference in weight from the two versions. This amounts to the difference between $830 \mathrm{~g}$ (the weight of the VLP-16) and $590 \mathrm{~g}$ (the weight of the VLP-16 Lite), a savings that amounts to a $240 \mathrm{~g}$. In addition, the version of VLP-16 used for the present study features a long cable, which adds to the weight of the system as well. The new version would feature the version with a shorter cable, thus saving extra weight. Hence, the developed system can easily have a weight saving of around $0.5 \mathrm{~kg}$, giving an overall weight of less than 2 $\mathrm{kg}$. This represents a $20 \%$ reduction in the system weight and would add to the mission flying time, which is critical in UAV-related tasks.

Other improvements have also been made. For instance, an easy and efficient way of mounting the developed system to the M600 has been implemented. The mounting mechanism chosen is a simple L-shaped angle bracket, along with an extra boxed shape just to add a layer of protection to the system while mounted. The simplicity of the mounting mechanism allows the developed system to be deployed to any other UAV without having to modify the platform. Another challenge that needed to be met is the interference the UAV multirotor and other UAV electronics components may pose on the developed system's GNSS antenna signal reception. In order to overcome this obstacle, the system's antenna needed to be elevated above the UAV electronics and rotors. This has been accomplished by a simple yet effective solution - by using a plastic cone. The plastic cone is lightweight but can still resist wind and maintain the stability of the system antenna. Further, its lightweight character will not add much weight to the max payload capacity. In addition, a metallic circular plate has been attached to the bottom of the system antenna to reduce the interference. The developed system mounted on the M600 is shown in Fig 6.1. 


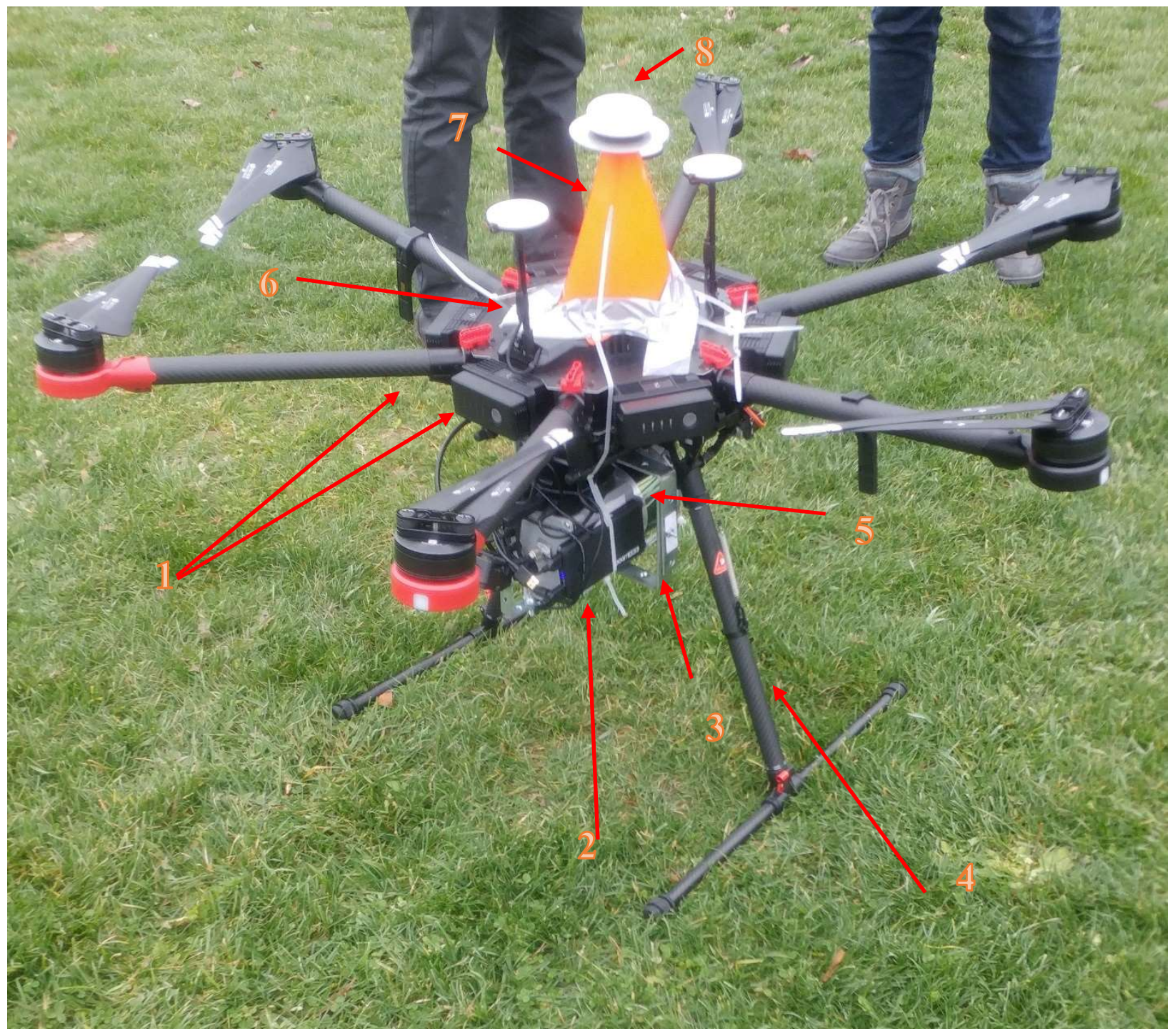

Fig 6.1: The System mounted in ULS mode on the DJI M600

1) The M600 \& batteries , 2) The developed mapping system , 3) box shaped cage, 4) M600 landing gears 5) The VLP-16, 6) The M600 antenna , 7) The plastic cone , 8) The system antenna

Figure 6.1 shows the various components of the ULS system modules and the M600 platform. As can be seen, the VLP-16 has been mounted with its spinning axis almost horizontal, so that the field of view will allow the mapping of the area under the M600 as it cruises. It is critically important to calculate the mounting angles and lever arms and provide those calculations to the APX-15 before the commission of the flying mission. 
Four sets of measurements need to be determined and entered, as follows:

1- The mounting angles from the Reference frame (the VLP-16 in the research at hand) to the IMU body frame;

2- The mounting angles from the Vehicle frame to the reference frame;

3- The lever arms between the Reference frame and the IMU body frame resolved in the Reference frame;

4- The lever arm between the Reference frame and the antenna phase center APC resolved in the vehicle frame.

These parameters need to be correctly determined and entered before commencing the flight mission, otherwise erroneous results would be reported by the POSPac from the SBET calculations. One of the obstacles and challenges that has been experienced in one of the field data collection mission is the drastically negative impact caused by EM interference. In one of the missions, a hyperspectral camera was mounted, with a large-size lithium-ion battery used as its power source. These items were deployed along the developed LiDAR mapping system, as depicted in Fig. 6.2 below.

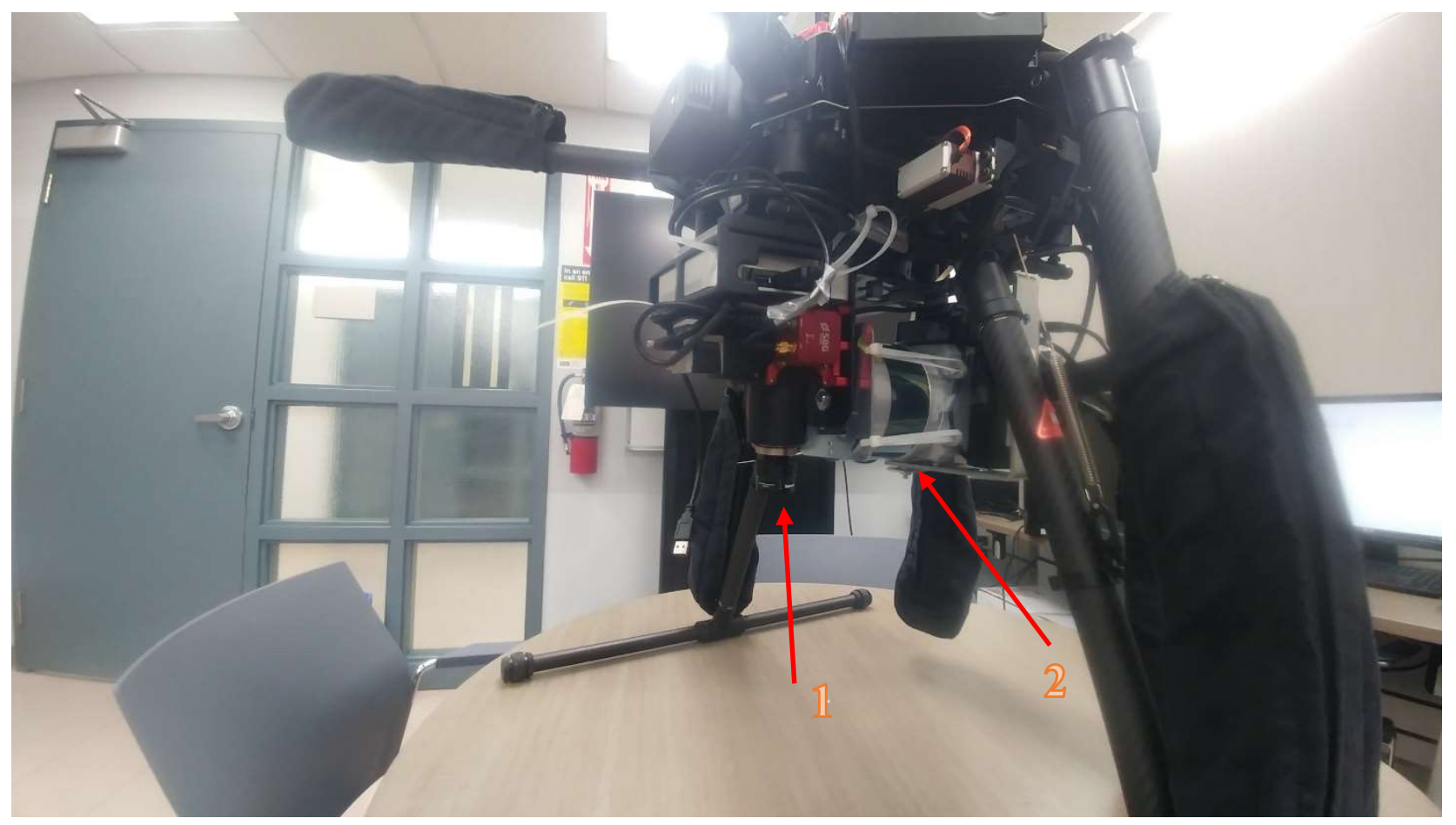

Fig 6.2: The System mounted in ULS mode with Hyperspectral camera

1) The Hyperspectral camera 2) The LiDAR-based system 
The effect of EMI is paramount on the GNSS signal reception, as demonstrated in Figs. 6.3 to 6.4

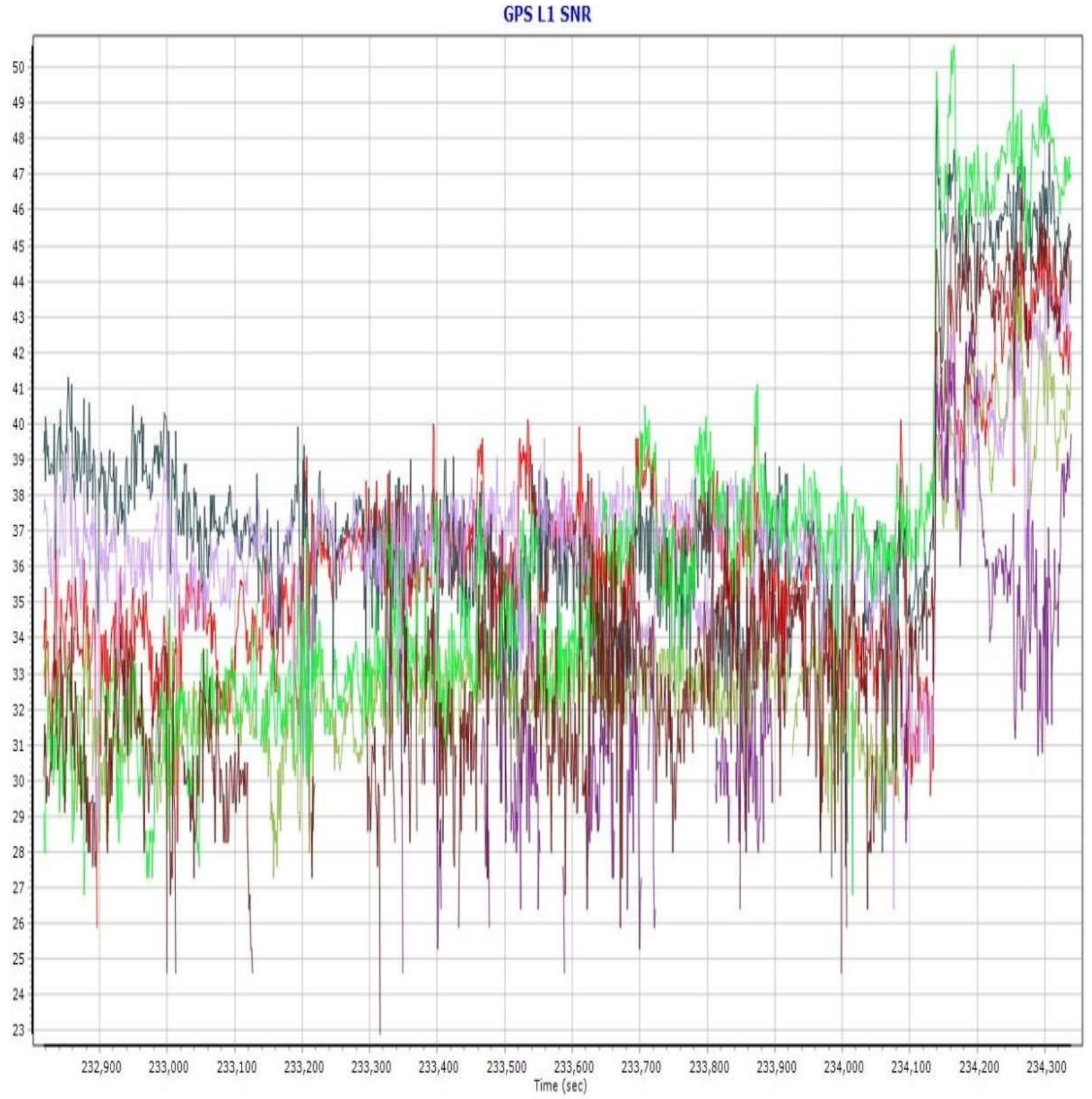

- GPS PRN 10 LI SNR (dB/Hz) - GPS PRN 15 LI SNR (dB/Hz) - GPS PRN 20 LI SIR (dB/Hz) - GPS PRN 21 LI SNR (dB/Hz) - GPS PRN 24 LI SNR (dB/Hz) - GPS PRN 27 LI SNR (dB/Hz) - GPS PRN 32 LI SNR (dB/Hz)

Fig 6.3: GPS L1 Signal to Noise Ratio 


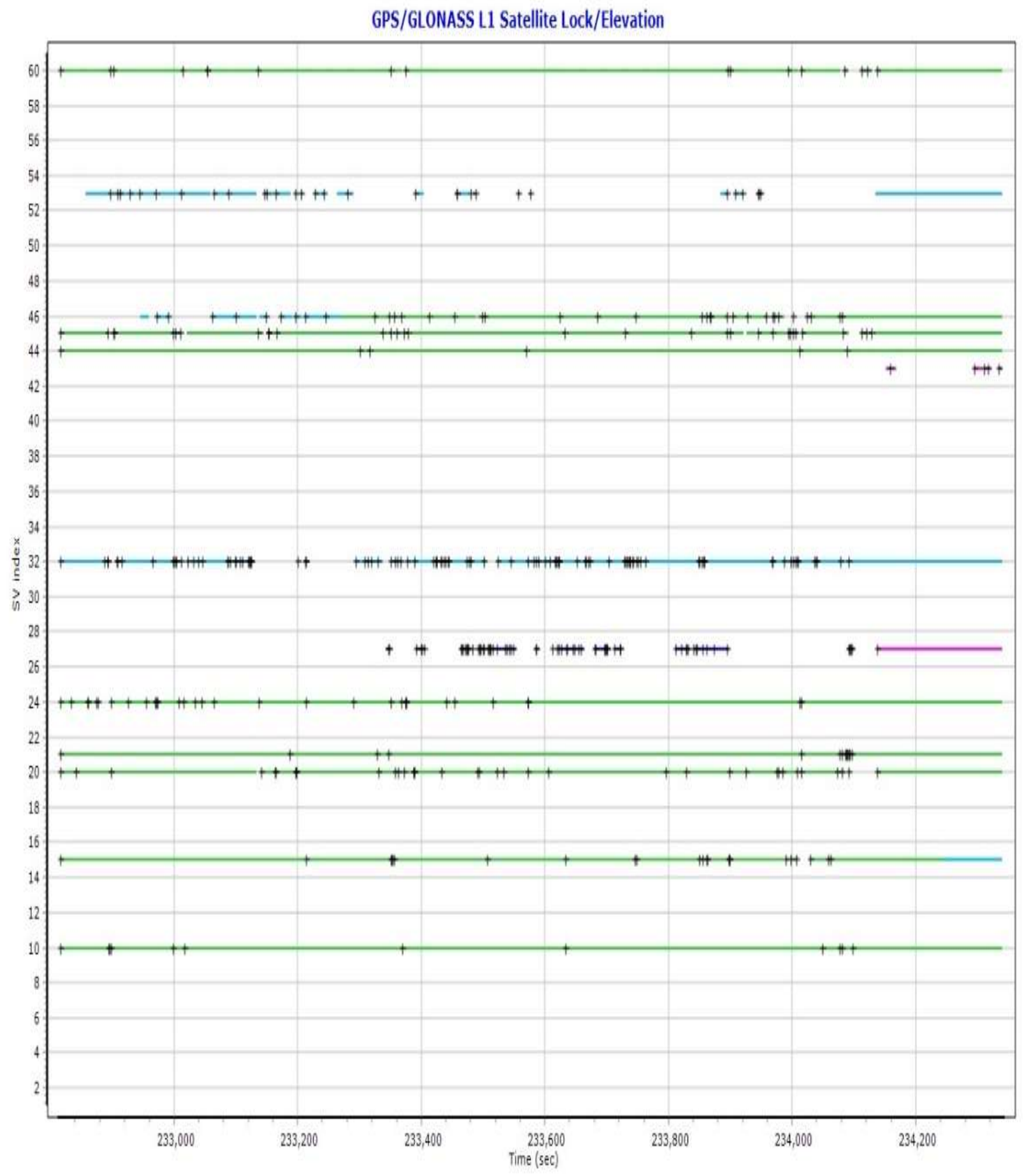

$-0.10 \mathrm{deg}-10 \cdot 15 \mathrm{deg}-15 \cdot 20 \mathrm{deg}=20 \cdot 30 \mathrm{deg}-30+\mathrm{deg}=$ No ephem + Cyde slip

Fig 6.4: GPS/GLONASS L1 Satellite Lock/elevation 
Fig 6.3 shows the GPS L1 Signal-to-Noise Ratio which appears to be lower than the expected average of $\sim 40 \mathrm{~dB} / \mathrm{Hz}$ during the flight. It returns to the average at the end, which appears to coincide with the switching-off of the large-size lithium-ion and the hyperspectral camera, suggesting these devices as the source of interference attenuating the GNSS signal for the developed system. In addition, Fig. 6.4 depicts the GPS/GLONASS L1 Satellite Lock/elevation, and it appears to show the occurrence of numerous cycle slips, which is not normal with satellites at $30^{\circ}$ or above relative to the horizon. The effect on the L2 signal is even more profound; in Fig. 6.5 , complete outage of satellites is observed.

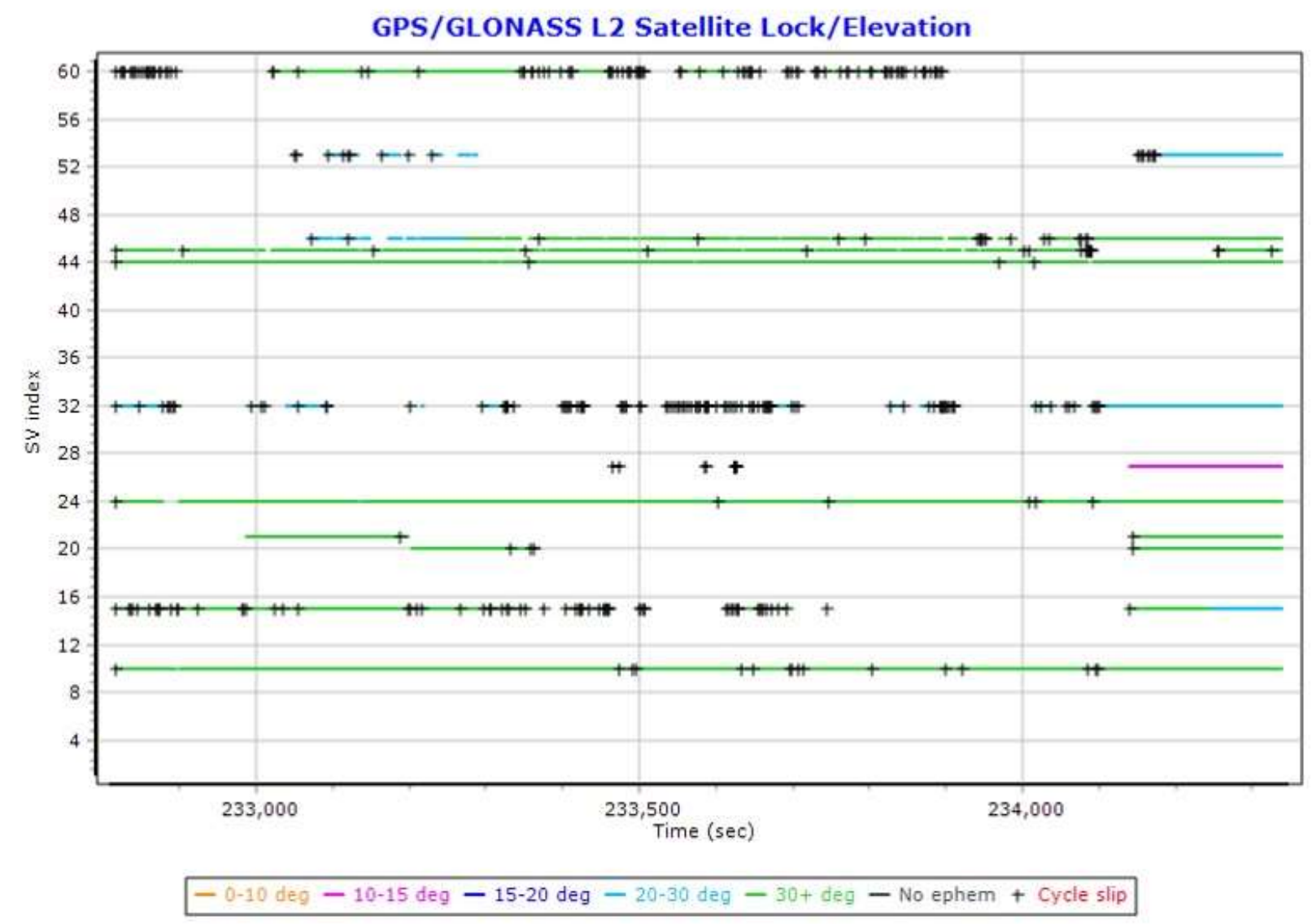

Fig 6.5: GPS/GLONASS L2 Satellite Lock/elevation

The effect of interference on the PPP solution is likewise apparent using the NRCAN PPP service, as shown in Figs. 6.6 and 6.7. 


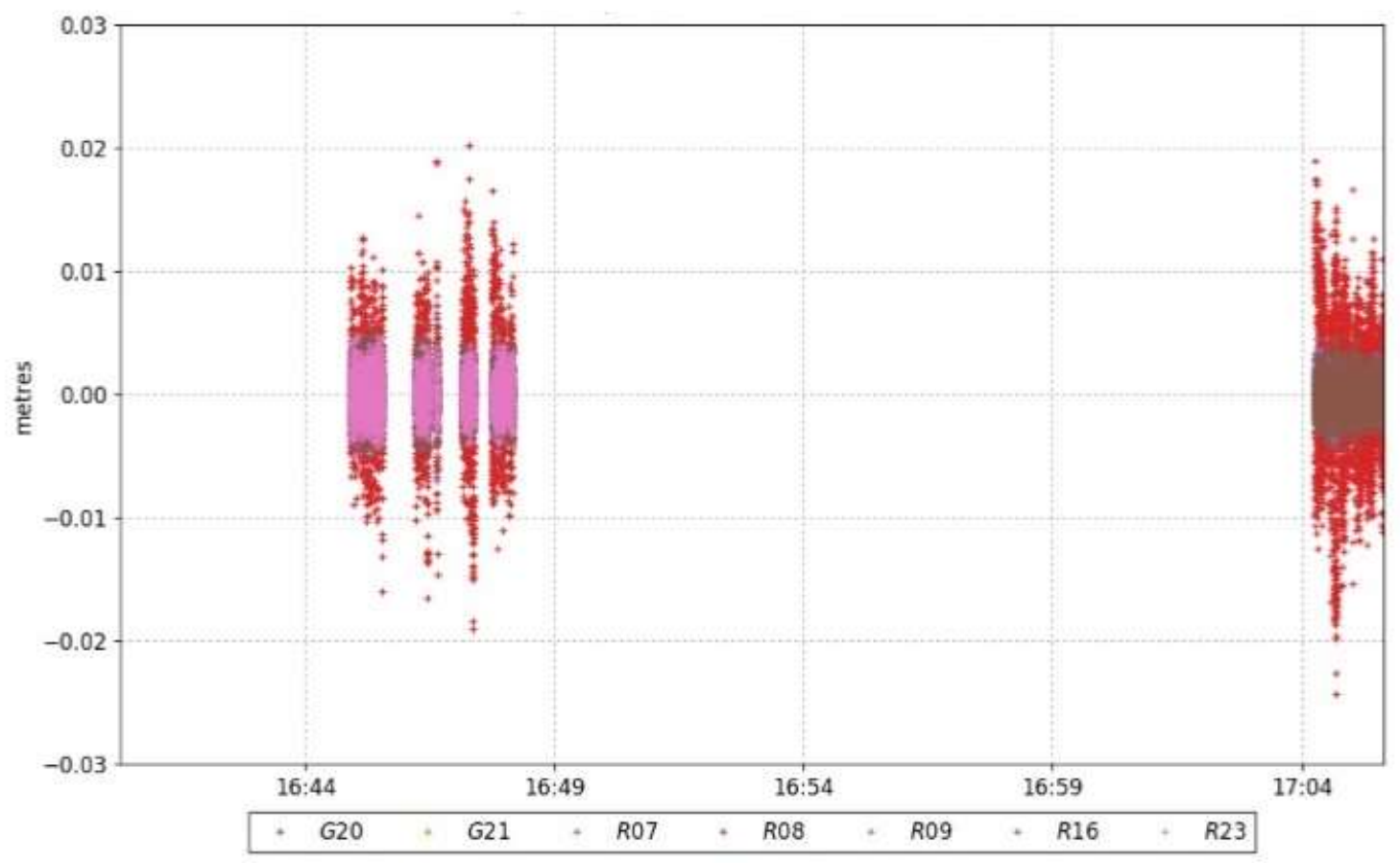

Fig 6.6: Carrier-Phase residuals

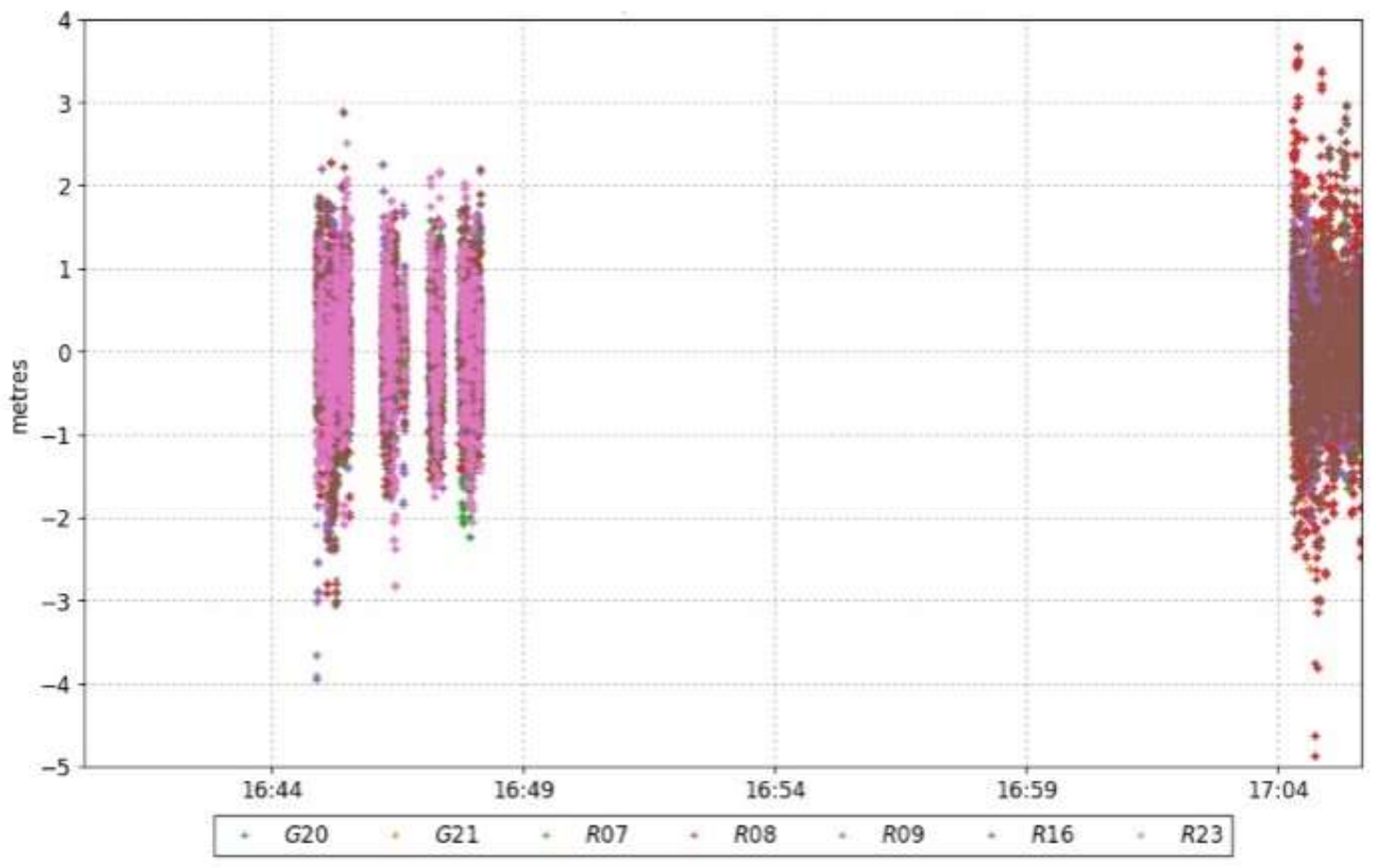

Fig 6.7: Pseudo-range residuals 
Analysis of the data presented in Figs. 6.3 to 6.7 reveals the drastically negative effect of EM interference on the GNSS signal reception, highlighting the importance of mitigating such interference. However, mitigation can be challenging, given the limited space available on board the UAV and the electronics and circuits that must be present.

\subsection{Pre-flight planning}

In order to ensure a successful flight mission, a number of factors need to be taken into consideration. These include parameters pertaining to the GNSS data, such as the number of satellites at the area of collection, DOP, availability, and other telemetry data of the GNSS constellations. In addition, weather conditions during the proposed flight time, such as wind, gusts, humidity and precipitation must be considered, along with other measures such as the K-index, which alerts for geomagnetic storms that might affect the GNSS signals. Other logistics measures also need to be taken into consideration according to the airspace type where the flight will take place (i.e., whether it is near an airport, hill port, a restricted airspace or not). Moreover, since optical imagery is also collected for the SfM analysis, the flight plan need to include a flight autopilot pre-defined trajectory, where the area under consideration for mapping is covered by overlapping imagery to maintain the front-lap and side-lap percentage of coverage.

As well, the APX-15 needs to be initialized to be able to define a local level frame of reference. This is achieved by applying cycles of accelerating and deaccelerating with hovering. In addition, flying in a box-shape flying pattern (Fig. 6.8) during the initialization process ensures a proper heading alignment.

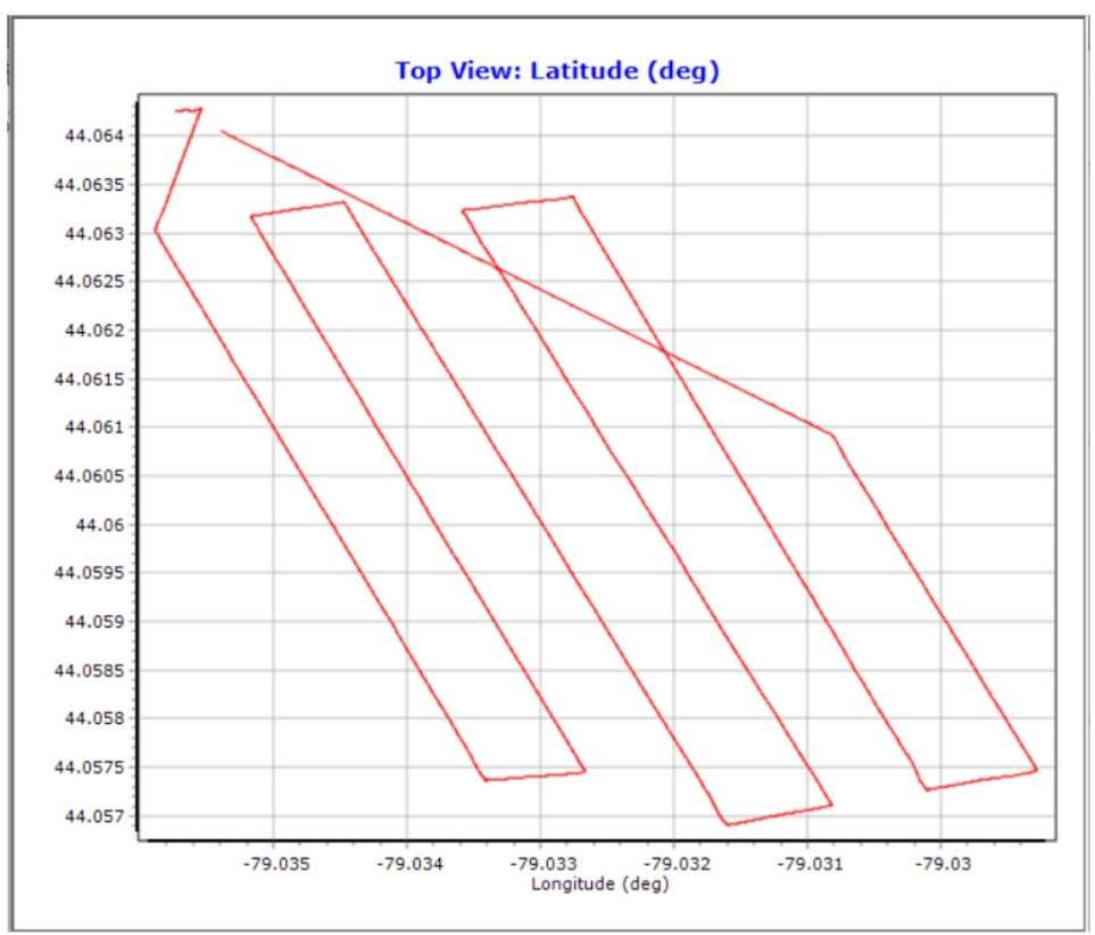

Fig 6.8: Box shape flying pattern for rotor UAV 


\subsection{System Deployed as ULS}

A ULS data collection mission has been planned and executed. The area mapped covers the Lion's Valley Park in Oakville, Ontario, Canada. A layout of the area covered is shown in Fig. 6.6. The area has been selected to test the performance of both the LiDAR-based developed system at hand and the SfM optical imagery workflow. The main features of the area are low vegetation, along with tall trees, a creek, and a pedestrian walking corridor. In addition, some metal sheds with a recognizable roof geometry are present.

As a first step, the system's components have been checked, including the power sources. The mounting angles between the APX-15, VLP-16, and M600 pro have been determined and programed into the APX-15 interface. In addition, the lever arm between the reference frame and APX-15 body frame has been measured (resolved in the VLP-16 reference frame), the lever arm between the APC and the VLP-16 reference frame has been measured (resolved in the vehicle (M600 pro) frame) and has been entered into the APX-15 configuration settings. All three coordinate reference systems for the APX-15, the vehicle, and the VLP-16 are right-handed coordinate systems. The VLP-16 has been mounted such that its Y-axis points down, its X-axis points to the starboard, and its Z-axis points forward. On the other hand, the APX-15 is mounted with its $\mathrm{Y}$-axis pointing down, its $\mathrm{X}$-axis pointing to the port, and its $\mathrm{Z}$-axis pointing down. The vehicle (M600 pro) has its $\mathrm{X}$-axis in the flight direction, its $\mathrm{Y}$-axis pointing to starboard, and its Z-axis pointing down. These mounting parameters will not change unless the system needs to be redeployed in another configuration.

The area covered is Lion's valley park in Oakville, Ontario. The location of the park is presented in Fig 6.9 


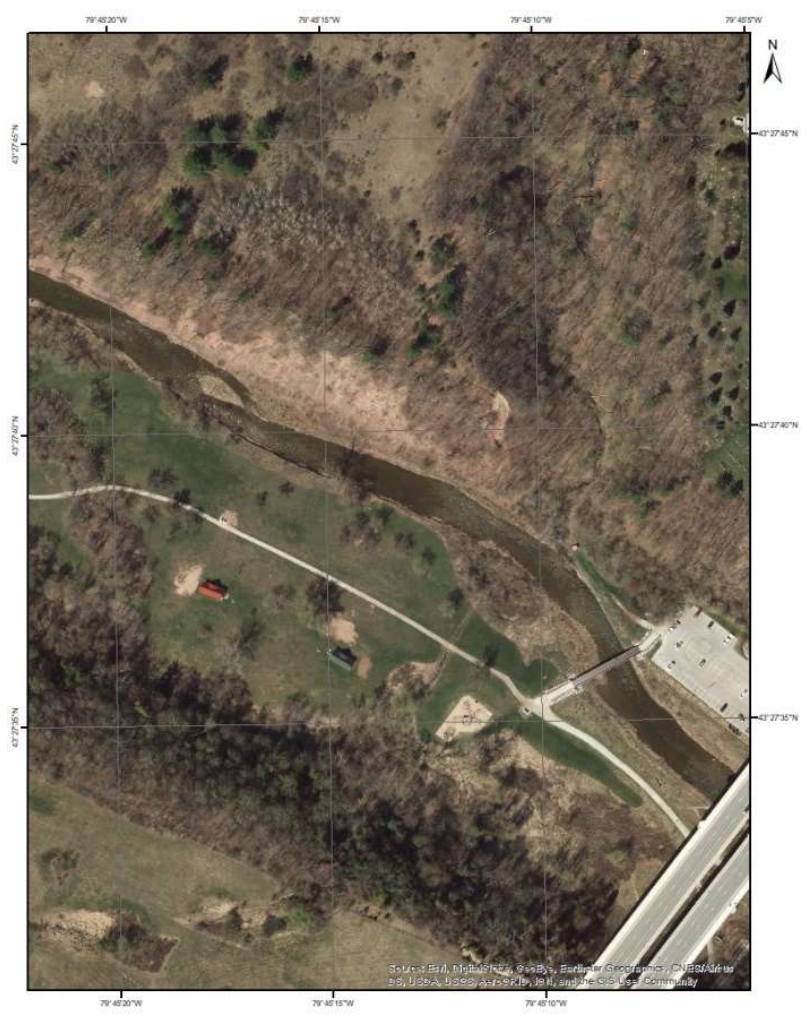

Fig 6.9: Lion's park area layout (mapped area with the system in ULS mode)

The location of the Lion's Valley Park is given in Fig. 6.9. As shown in the figure, the mapped area includes tall and short trees, a water creek, a walking path, and sheds. A base station was set at the area using a TRIMBLE R9S GNSS receiver and a Trimble Zephyr 2 antenna. The base station recorded its static position for three hours for post-processing analysis. The system components were rechecked at the field site. The APX-15 initialization was performed and the POS data recorded onboard the APX-15, while the VLP-16 data was recorded on the system control unit for further post-processing.

The overall area has been mapped by the developed system at hand, deployed on the M600 pro UAV. The flying height was set to $20 \mathrm{~m}$ and a number of overlapping flight strips were flown to map the area. In addition, the DJI Phantom 3 professional drone was utilized for the optical imagery data capture needed for the SfM workflow analysis. The DJI Phantom 3 specifications are shown in Table 6.3, while the specifications for the camera onboard the drone are shown in Table 6.4 As can be seen in the figure, there is a huge difference in size between the M600 pro and the LiDAR system deployed, relative to the Phantom 3 professional drone. 
Table 6.3: Phantom 3 professional specifications (AIRCRAFT)

\begin{tabular}{|l|l|}
\hline \multicolumn{2}{|c|}{ Attribute } \\
\hline Max Flight Time & Approx. 23 minutes \\
\hline Satellite Positioning Systems & GPS/GLONASS \\
& \\
& Vertical: \\
& $\pm 0.1 \mathrm{~m}$ (with Vision Positioning) \\
& $\pm 0.5 \mathrm{~m}$ (with GPS Positioning) \\
Hover Accuracy Range & Horizontal: \\
& $\pm 0.3 \mathrm{~m}$ (with Vision Positioning) \\
& \pm 1.5 m (with GPS Positioning) \\
& \\
\hline
\end{tabular}

Table 6.4: Phantom 3 professional specifications (Camera)

\begin{tabular}{|l|l|}
\hline \multicolumn{2}{|c|}{ Attribute } \\
\hline Sensor & $\begin{array}{l}1 / 2.3^{\prime \prime} \text { CMOS } \\
\text { Effective pixels: } 12.4 \mathrm{M} \text { (total pixels: } 12.76 \mathrm{M})\end{array}$ \\
\hline Lens & FOV $94^{\circ} 20 \mathrm{~mm}(35 \mathrm{~mm}$ format equivalent) f/2.8 focus at $\infty$ \\
\hline Electronic Shutter Speed & $8-1 / 8000 \mathrm{~s}$ \\
\hline Image Size & $4000 \times 3000$ \\
\hline Still Photography Modes & $\begin{array}{l}\text { Single Shot } \\
\text { Burst Shooting: 3/5/7 frames } \\
\text { Auto Exposure Bracketing (AEB): 3/5 bracketed frames at } \\
0.7 \text { EV Bias } \\
\text { Timelapse }\end{array}$ \\
\hline \multirow{3}{*}{ Video Recording Modes } & $\begin{array}{l}\text { UHD: 4096x2160p 24/25, 3840x2160p 24/25/30 } \\
\text { FHD: } 1920 \times 1080 \mathrm{p} 24 / 25 / 30 / 48 / 50 / 60 \\
\text { HD: } 1280 \times 720 \mathrm{p} 24 / 25 / 30 / 48 / 50 / 60\end{array}$ \\
\hline
\end{tabular}




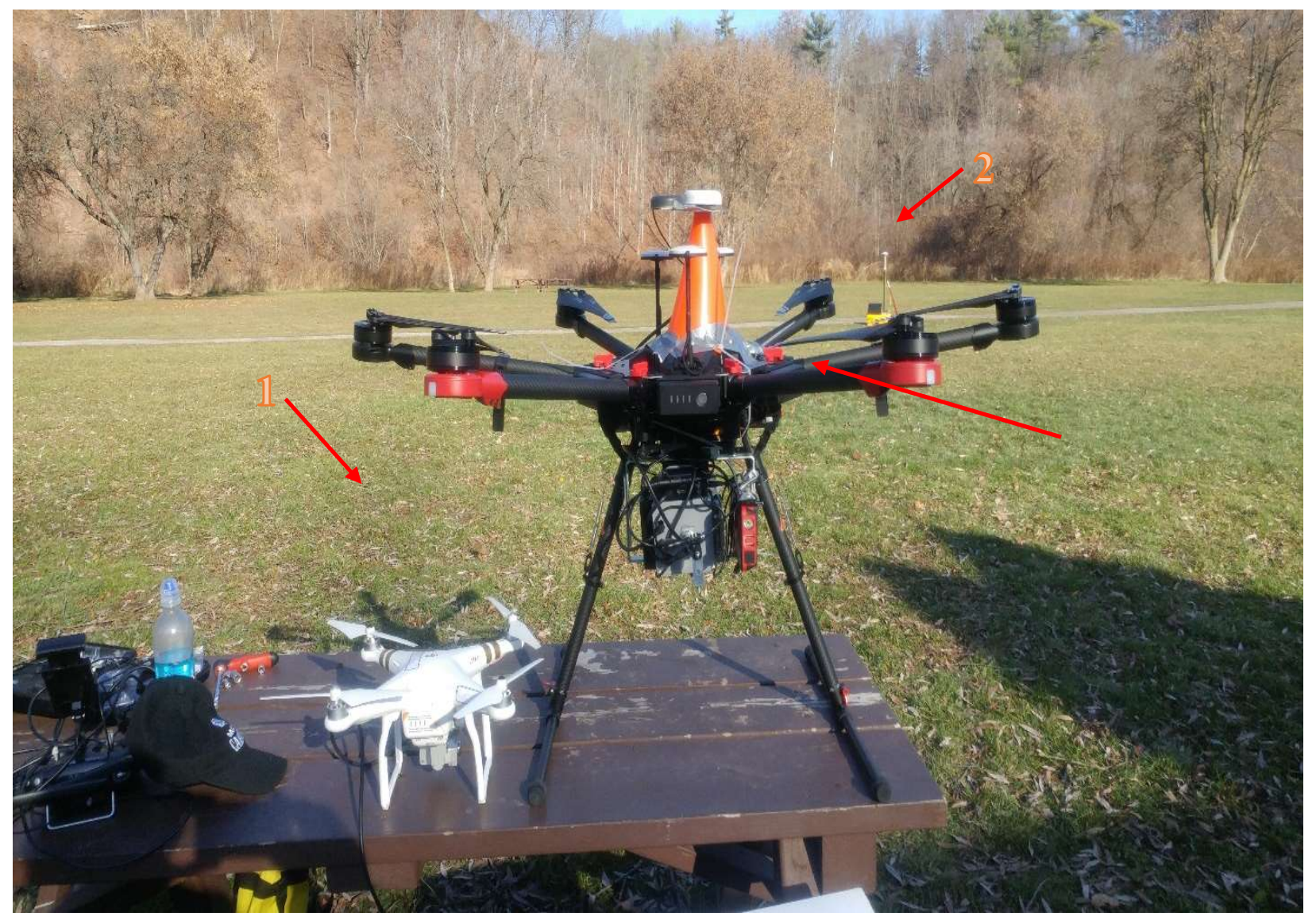

Fig 6.10: The system in ULS mode

1) Phantom 3 Professional 2) Base station

Fig 6.10 shows the huge difference in size between the M600 pro and the LiDAR system deployed relative to the phantom 3 professional drone. 


\subsubsection{LiDAR data collection}

The developed system has been mounted on the M600 pro, the on-site pre-flight check has been performed, and the APX-15 has been initialized at the field site, as described in section 6.1. The hardware connections between the different components of the mapping system and the UAV platform have been made. The VLP-16, which is a state-of-the-art, lightweight, compact-size LiDAR scanner, is a time-of-flight laser scanner. Unlike laser scanners that use a mirror for data collection, the VLP-16 consists of 16 pairs of emitters and receivers that rotate to cover $360^{\circ}$ around the scanner, thus providing 3D real-time scanning of the environment. The scanner can measure 300,000 points/sec., enabling it to map an area while mounted on the M600 pro. Further, the VLP-16 16 beams have been oriented to be at nadir by mounting the scanner with its spinning axis almost horizontal. The form of mounting allows the device to capture the area under the UAV, with a swath width depending on the LiDAR range

Although the advertised range for the VLP-16 is $100 \mathrm{~m}$, practical testing shows that the typical range is around $60 \mathrm{~m}$. It is probable that the $100 \mathrm{~m}$ advertised range occurs with highly reflective objects. The geometry of the VLP-16 capturing the area mapped is depicted in Fig. 6.11. As shown in the figure, the 16 beams cover 16 circular arcs due to the rotation in $360^{\circ}$. Also, since the sensor is, with its spinning axis, almost horizontal, the 16-beam coarse vertical resolution is highlighted by the distance between the beams, as shown in Fig. 6.11. As the UAV cruises, the gaps are filled by another set of firings for the 16 beams until the area of interest is mapped.

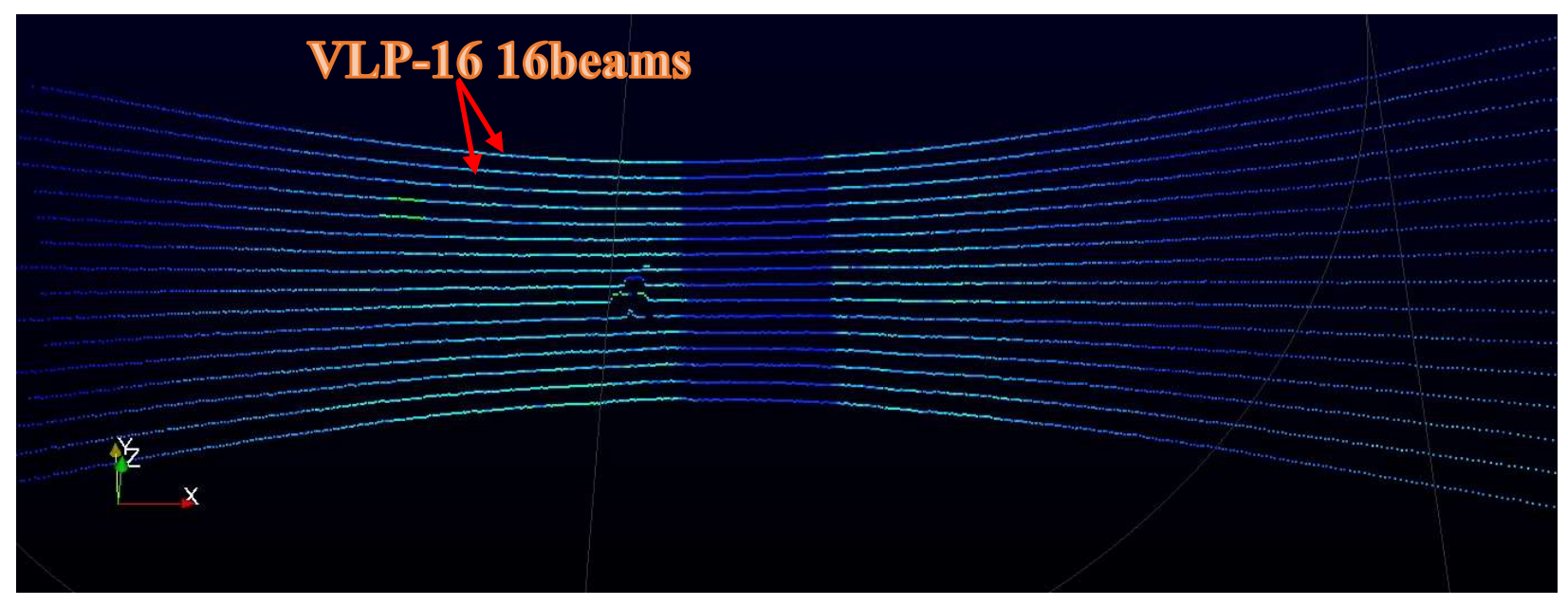

Fig 6.11: The VLP-16 beams and the gaps in-between 


\subsection{Optical Data collection}

The DJI phantom 3 professional has been used for the optical imagery data collection. After doing the flight plan to cover the area so the trajectory would be fed to the autopilot and maintain the time requirements for the DJI battery running time, the frontlap has been set to $70 \%$ and the sidelap to $50 \%$. The flying mission to capture the overlapping images to cover the area of interest and maintain the $50 \mathrm{~m}$ height to be safe from possible collisions with tall trees, lasted around 15 minutes.

The trajectory of capturing the images and to maintain the front and side lab is shown in Fig 6.12

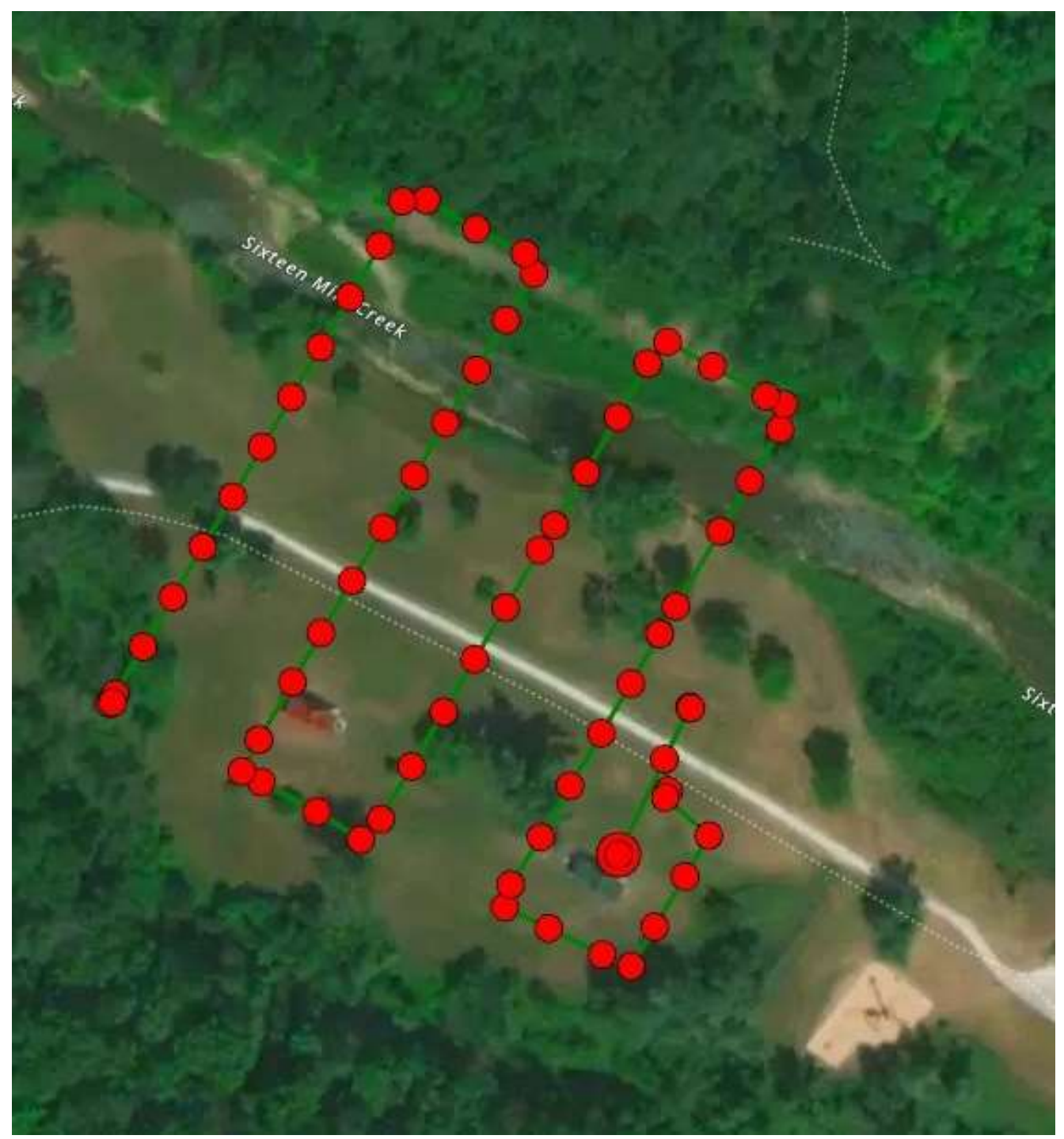

Fig 6.12: DJI autopilot imagery capture trajectory

A sample of the imagery captured is presented in Fig 6.13 


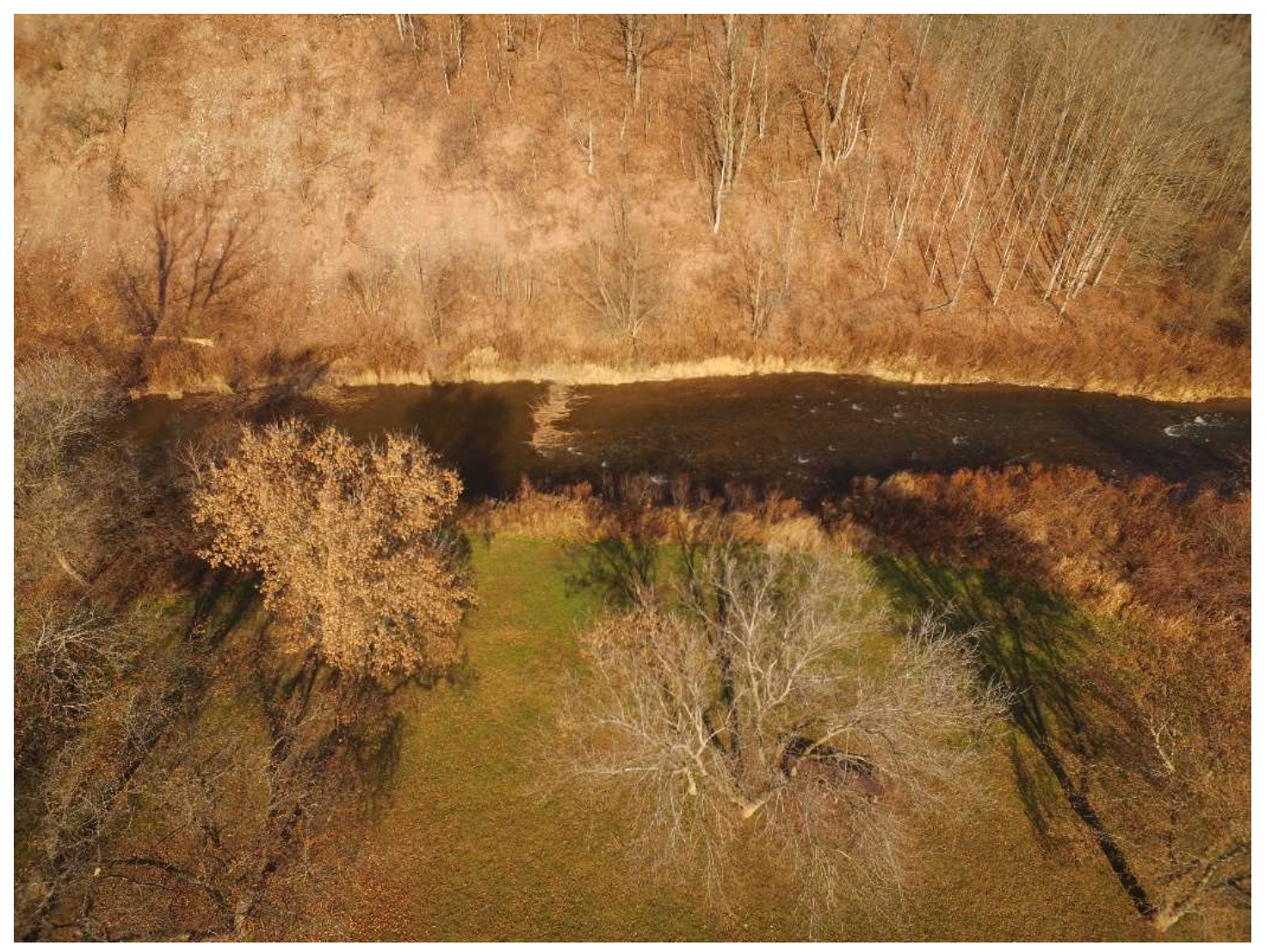

Fig 6.13: DJI sample imagery captured

Although the DJI Phantom 3 Professional is a mid-cost drone, it has a powerful stabilized imagerycapturing mechanism, which is evident by checking the sample imagery captured

\subsection{Captured Data Analysis (LiDAR)}

The VLP-16 data is time-stamped and synchronized by the APX-15 PPS signal. The APX-15 and GNSS data are then combined and adjusted by the base station readings to produce the SBET of the trajectory of the data collection mission. The data streams from the GNSS, the APX-15 and the VLP-16 are then joined and the DG equation is applied to produce the georeferenced LiDAR point cloud of the area. The processing of the LiDAR data and its fusion with the POS readings follows the procedure described in section 3.4 and summarized in the workflow shown in Fig. 3.10 .

The lay-out of the M600 trajectory and the performance metrics of the SBET file are shown in Figs. 6.14 to 6.20 . 
Top View: Latitude (deg)

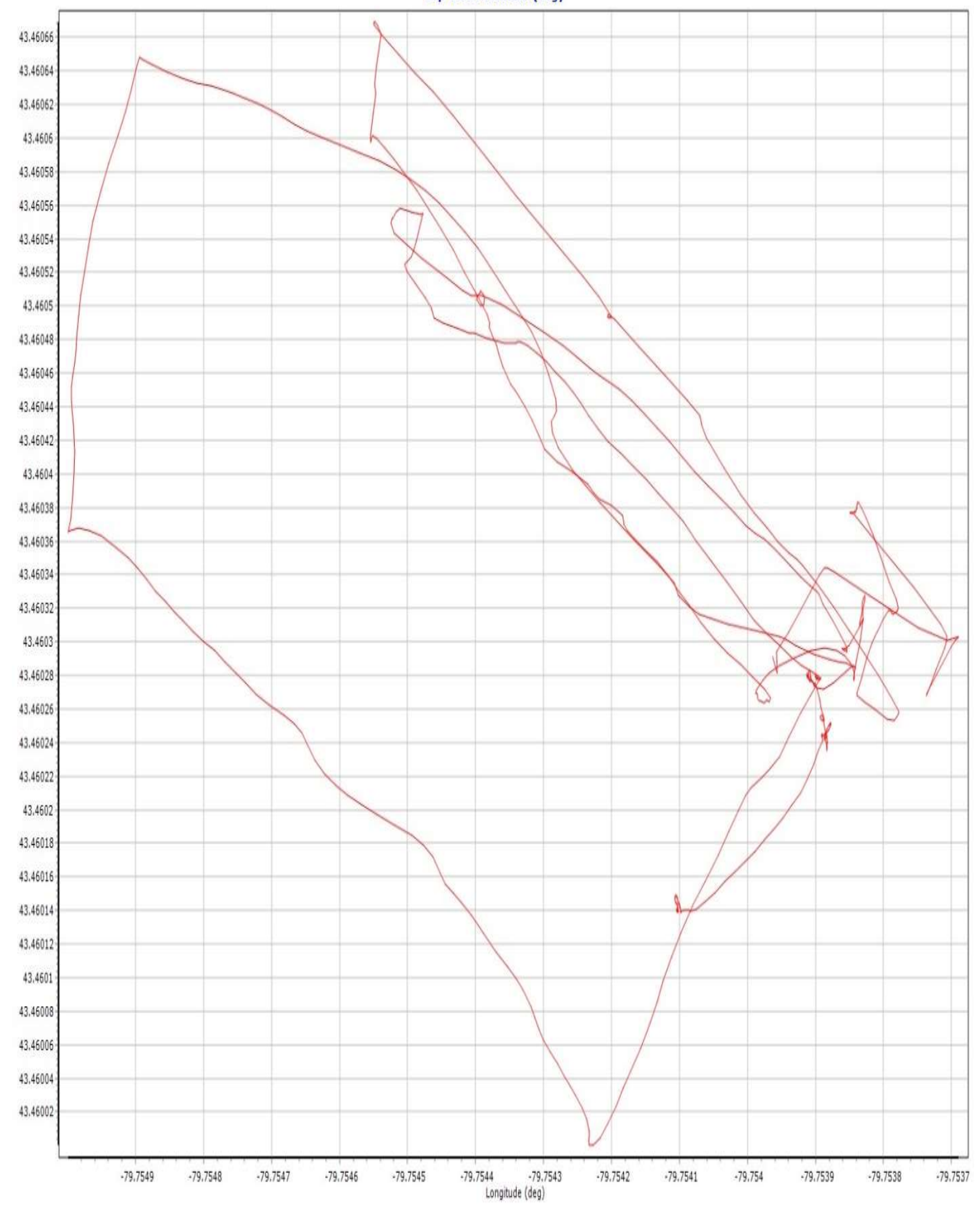

Fig 6.14: A top view of the M600 trajectory 


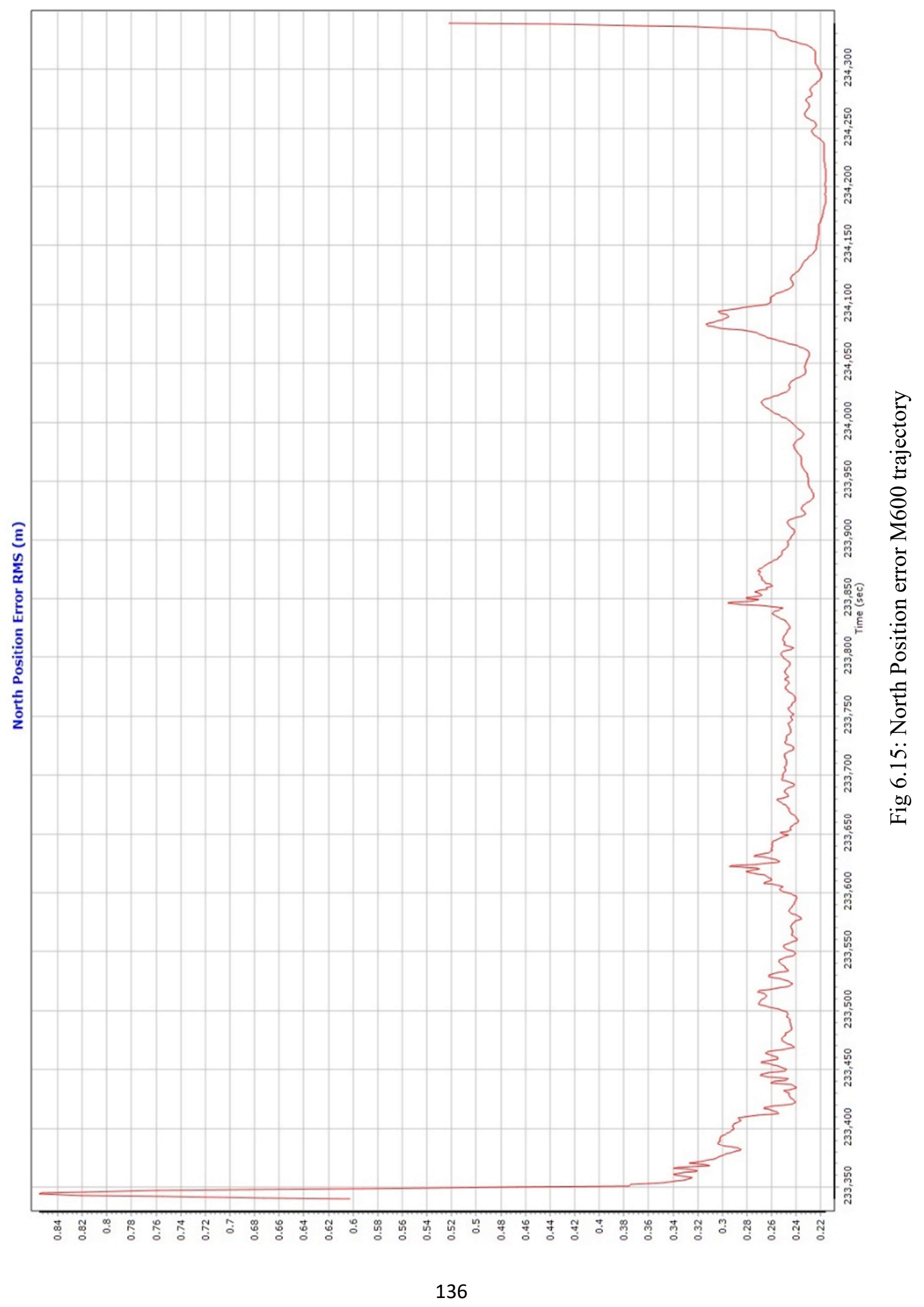




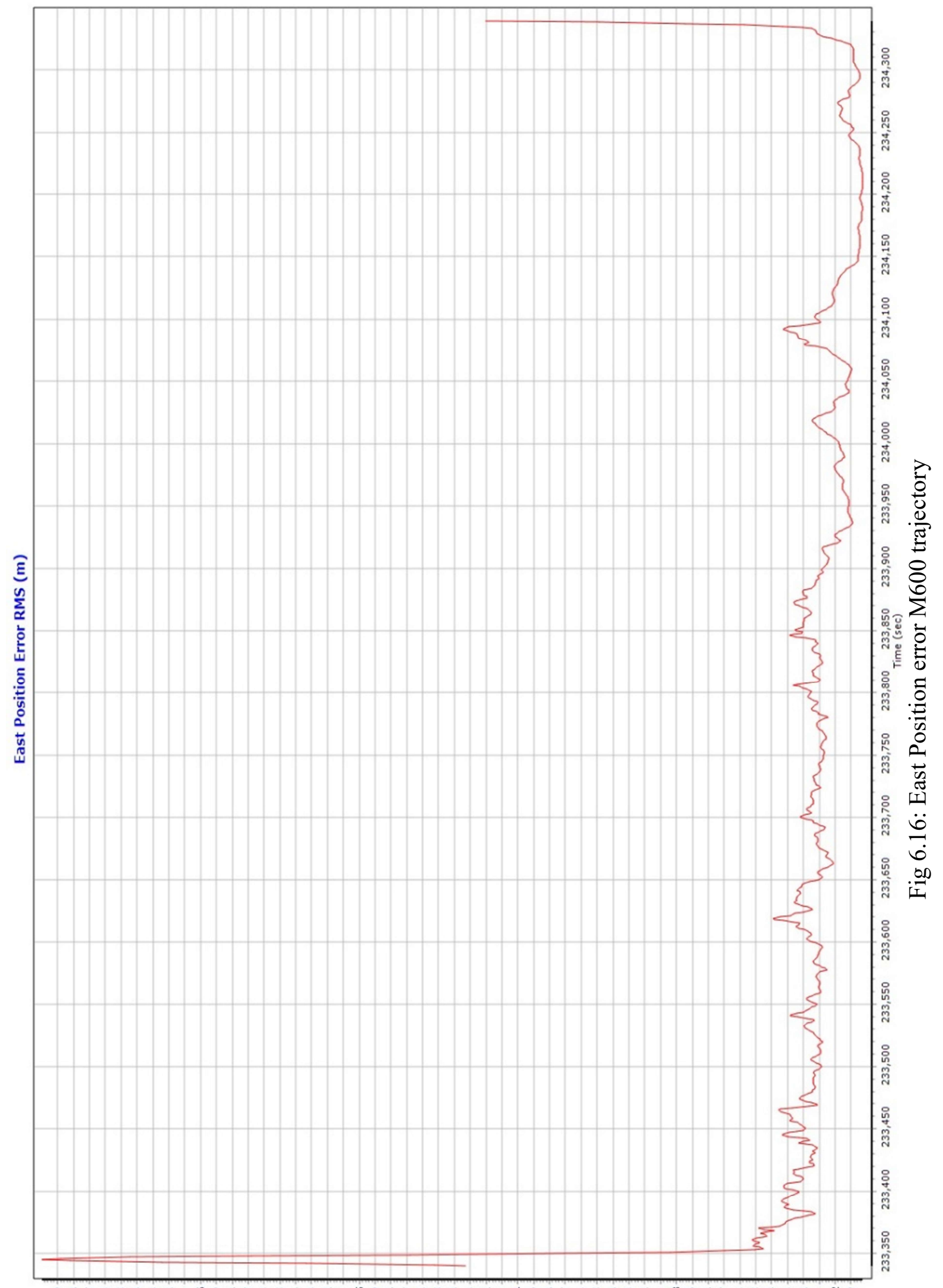

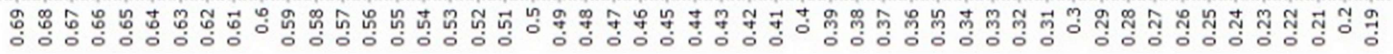




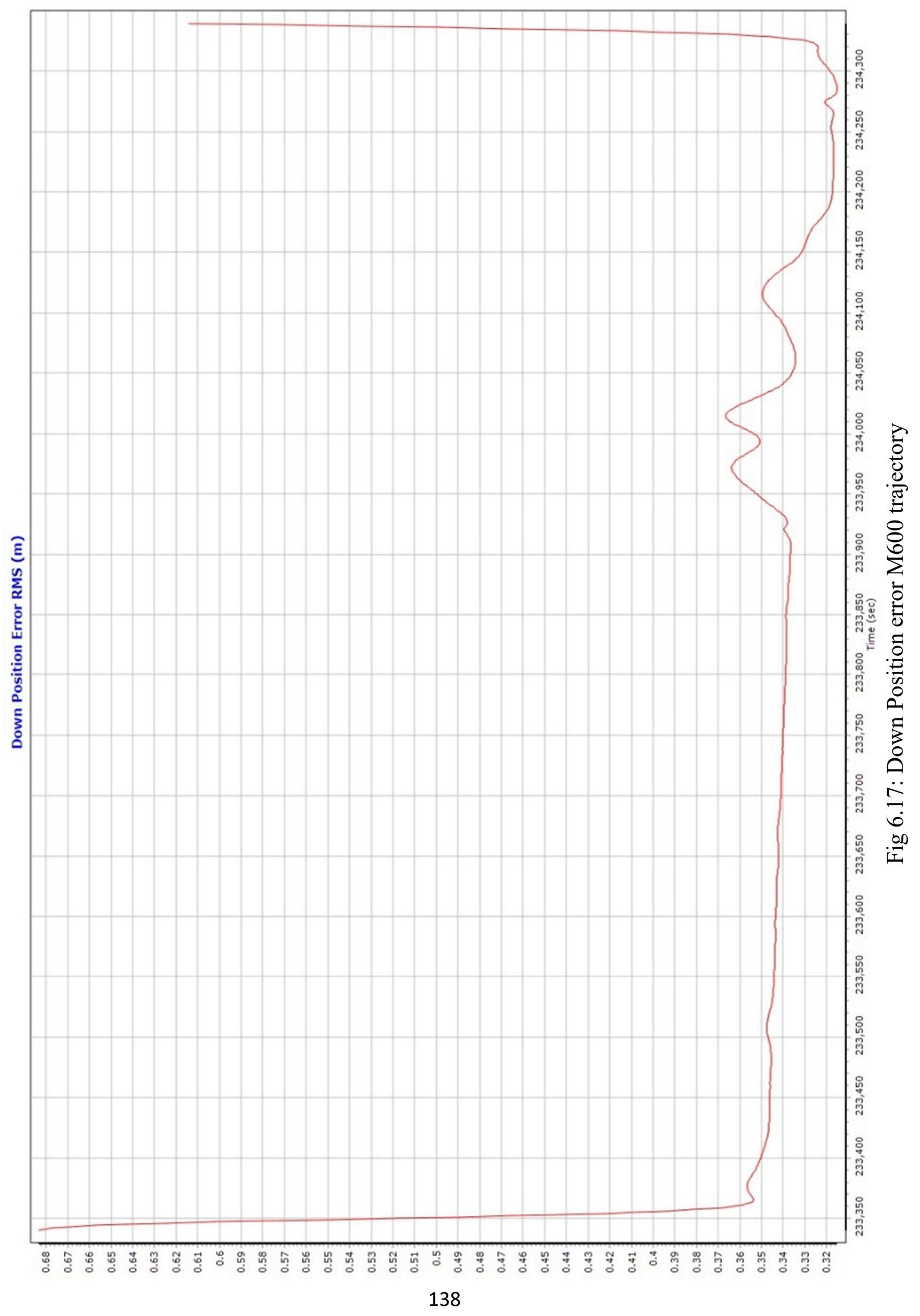




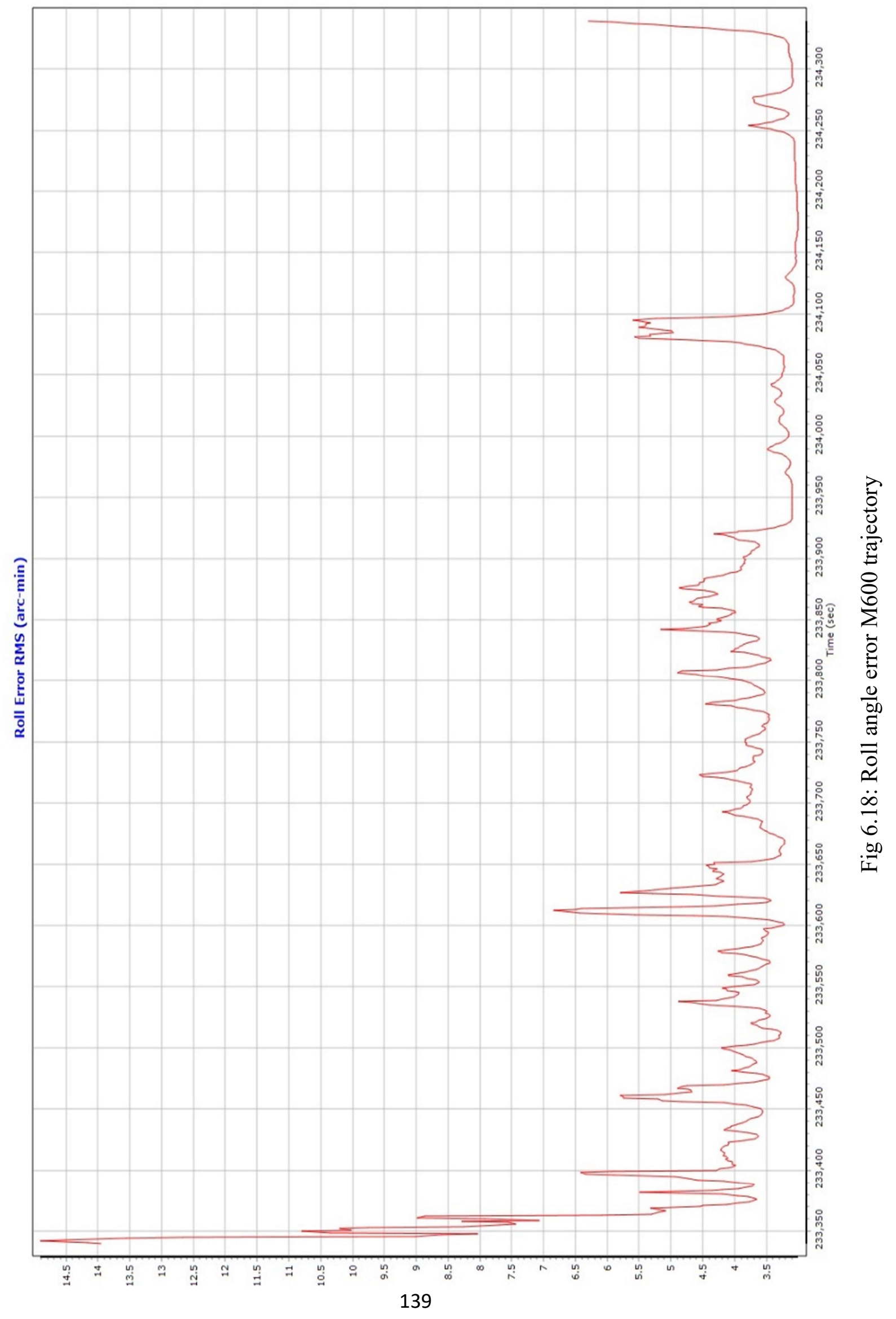




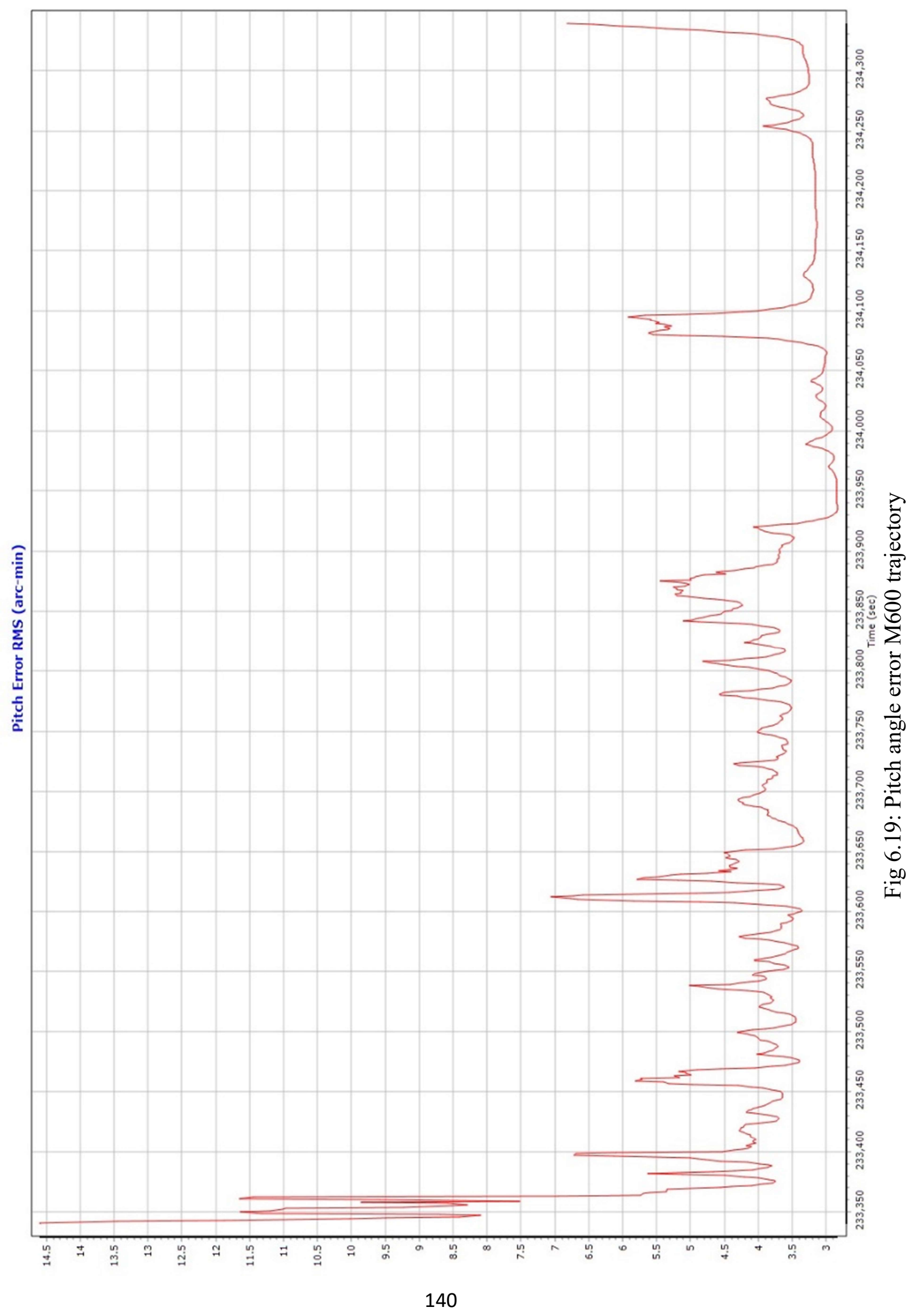




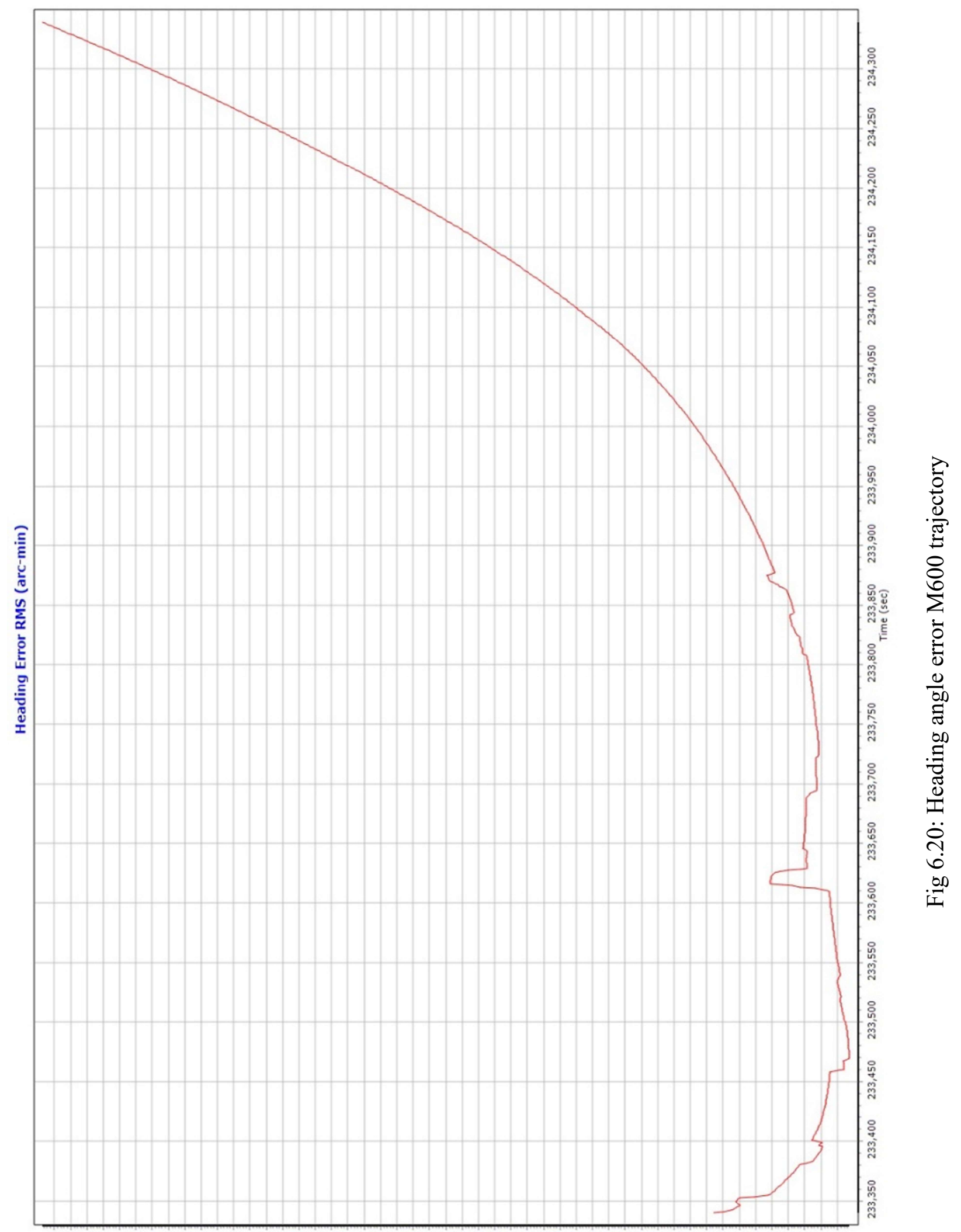

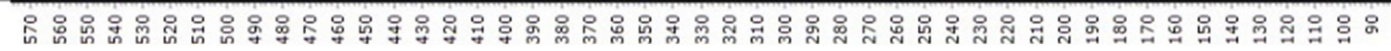


From Figs. 6.14 to 6.20, we can see that the SBET performance metrics showed the least precision in the heading angle and the down position, respectively. The precision reported is considered relative, as the base station was set at point with unknown coordinates.

The georeferenced LiDAR data are shown in Fig. 6.21 below

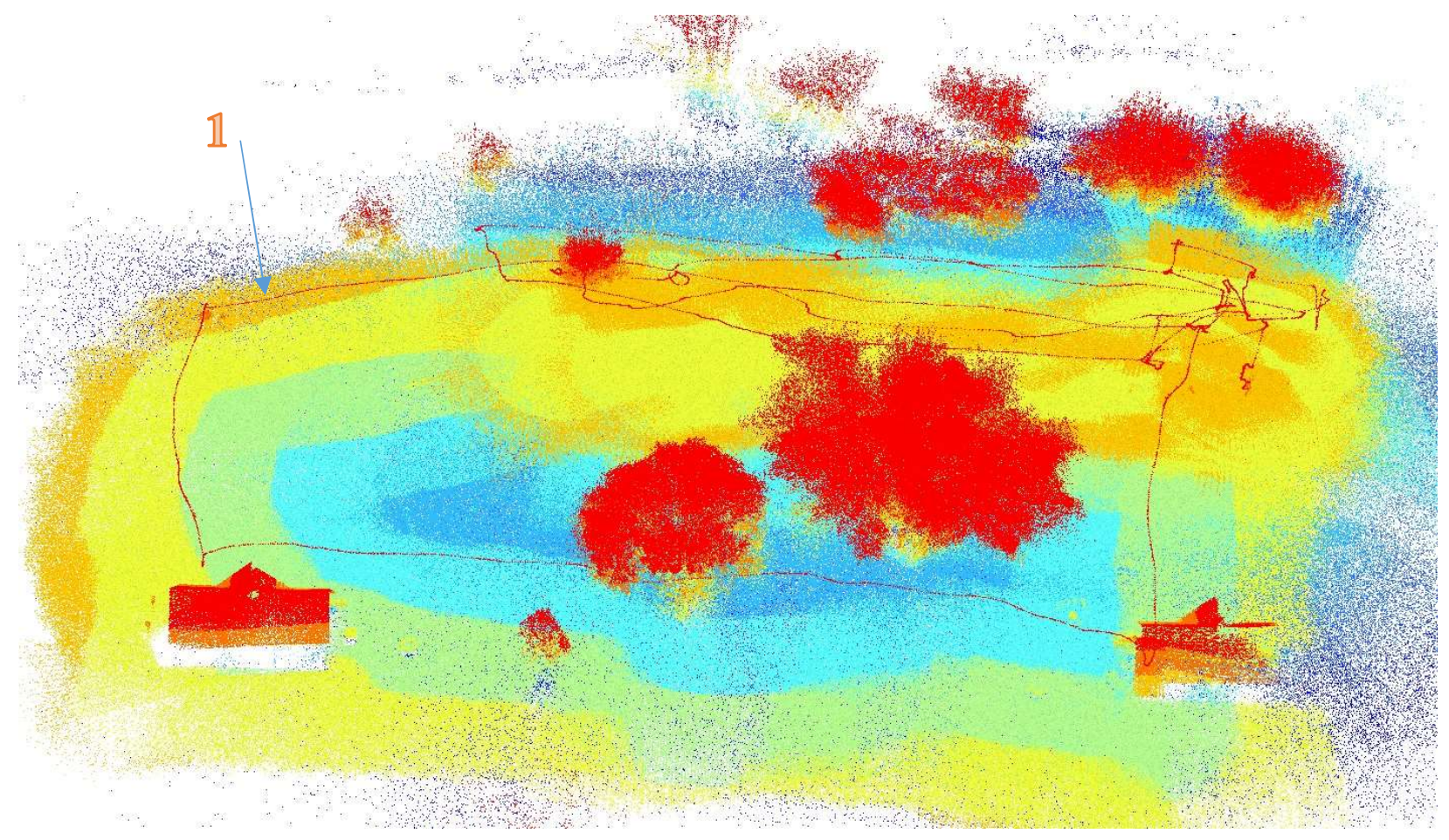

Fig 6.21: The geo referenced LiDAR data

The track numbered 1 in Fig. 6.21 is simply the result of the LiDAR scanner beams when rotating $360^{\circ}$. Because of the mounting setup deployed, the beams hit the M600 Pro's belly and thus map the trajectory followed by the M600 Pro. It serves as check for the georeferencing process and to highlight the trajectory within the resultant point cloud. If the resultant data need to be visualized without the track of the M600 Pro, the point cloud can easily be edited and the track removed. Checking the data presented in Fig. 6.20, the qualitative assessment of the developed mapping system performance at hand shows very good results. The only drawback is the effect of the boresight and lever arm misalignment, resulting in a deviation between same mapped objects from different strips which can be alleviated by performing a specialized system calibration. 
A sample of the data collected and the fine details mapped is shown in Fig 6.22 below.

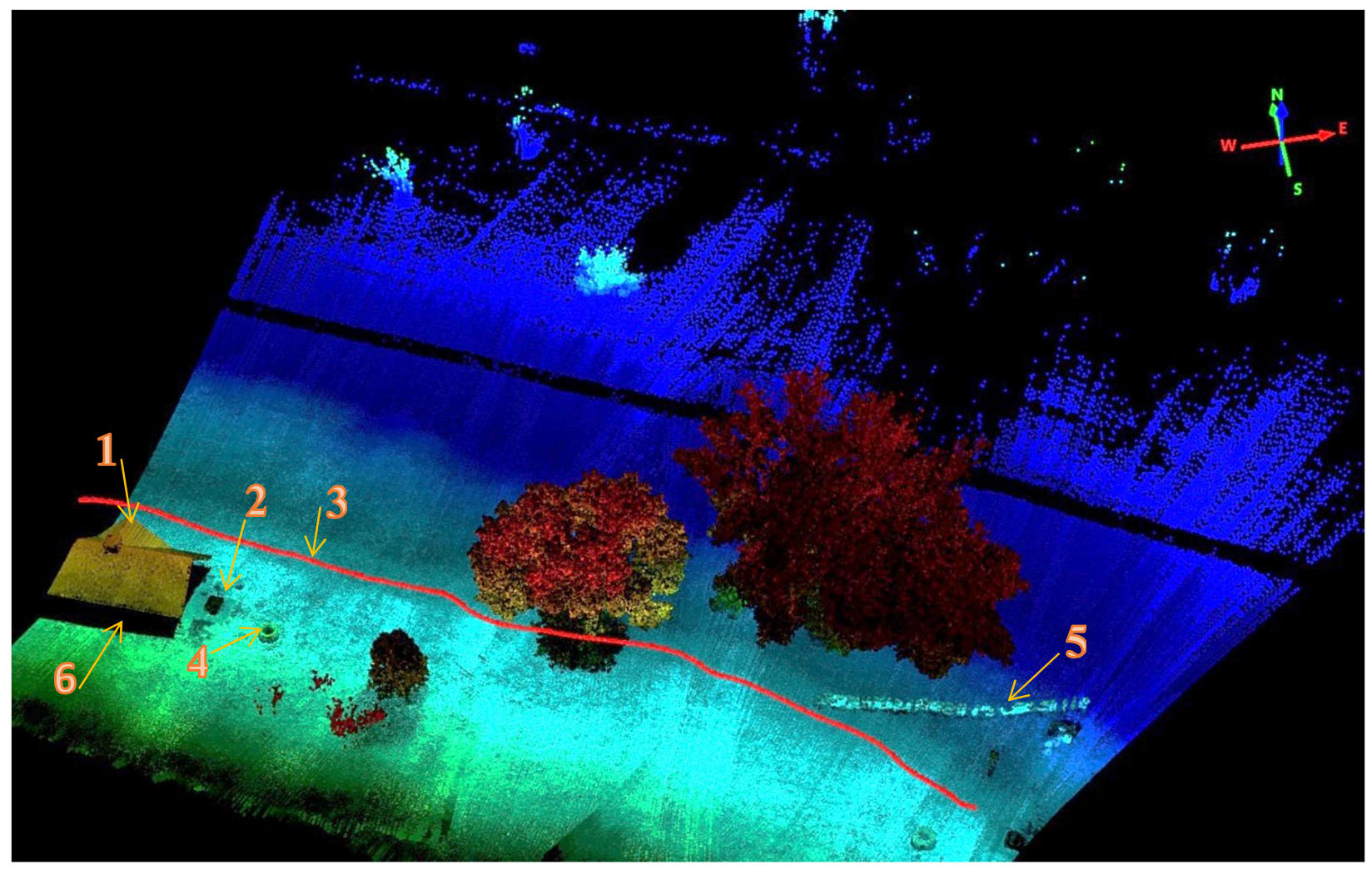

Fig 6.22: Sample from Georeferenced point cloud

Figure 6.22 clearly shows the ability of the developed system to map fine details, which are marked and highlighted in the figure, as follows:

1- The Shed

2- The BBQ

3- Part of the trajectory from the reflected laser beams on the M600 belly

4- The BBQ ash bin

5- A moving person

Figure 6.22 also shows an empty portion of the point cloud which follows the walking path present in the mapped area. The lack of data in this area might be attributed to the path being wet at the time of the LiDAR mapping. The LiDAR operates in the NIR wavelength range of $905 \mathrm{~nm}$, which is absorbable by water and which might explain this behavior. It is also important to point out the presence of shadows within the mapped area. Specifically, the LiDAR data may get reflected by an object, and the area behind that object would not be mapped, as it has been shadowed.

In order to quantitatively assess the noise present in the data, the georeferenced 3D point cloud forming the southern side of the shed (shown in Fig. 6.22 above) was investigated. A plane was fitted to the 3D point cloud using RANSAC, and a mesh was created from the fitted plane. The normal distance between the points and the mesh surface was then calculated and the resulting distances are color-coded and presented in Fig. 6.23. A histogram of the point distances to the 
mesh surface is shown in Fig. 6.24. As can be seen in the figure, the histogram follows a normal distribution curve with a mean value equal to almost zero; the standard deviation of the point distances to the mesh surface has been found to be $4 \mathrm{~cm}$. This is a very good result, considering that the VLP-16 range precision is $3 \mathrm{~cm}$. Thus, having the $3 \mathrm{D}$ georeferenced point cloud deviate only $4 \mathrm{~cm}$ to the fitted surface highlights the successful deployment of the developed system in ULS mode and the high precision it attains. The results are also shown in Fig. 6.23, which presents the points color-coded by the deviation distance. This has been qualitatively proven by the fine details the system has been able to map, as illustrated in Fig. 6.22 above.

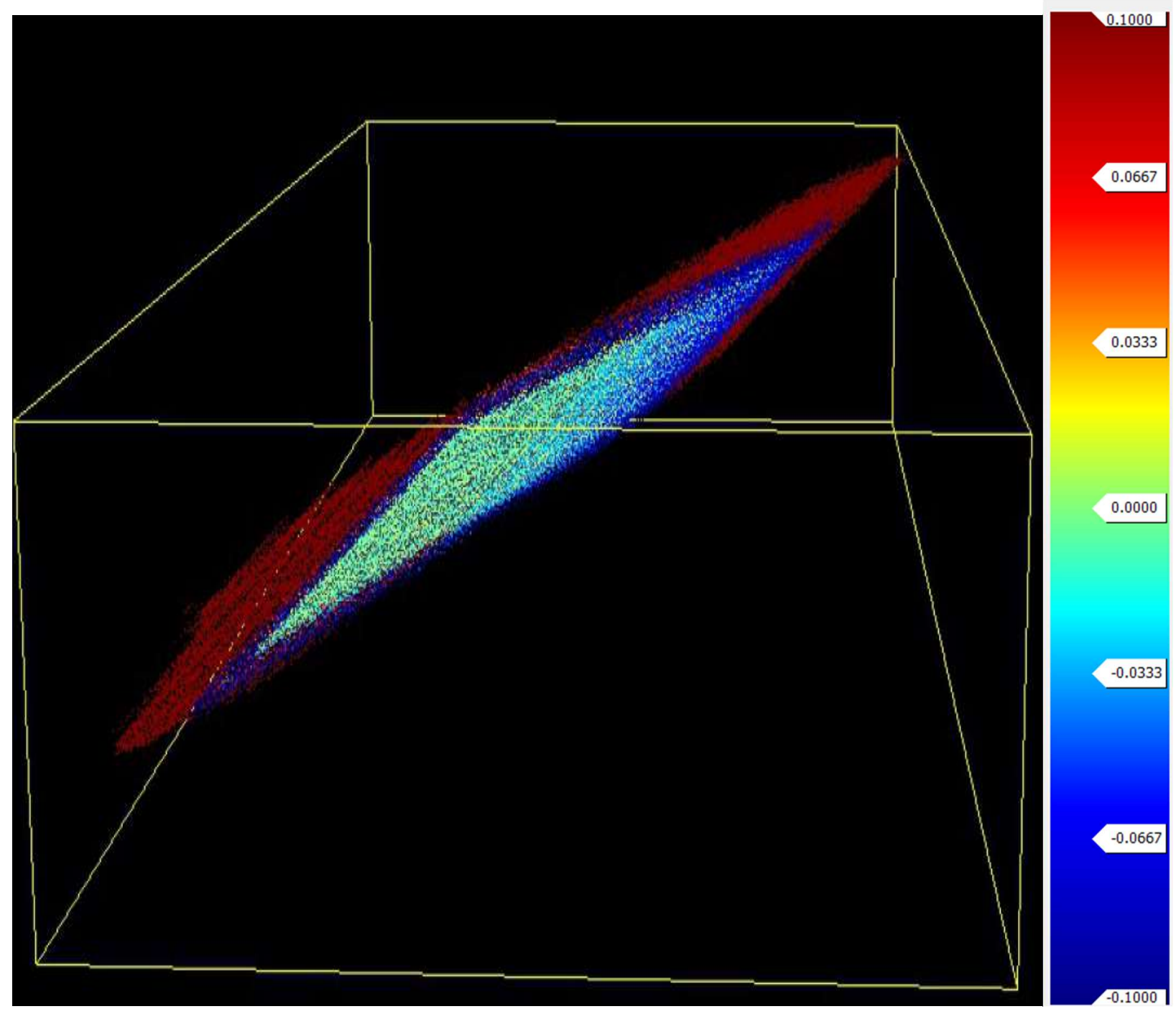

Fig 6.23: Georeferenced 3D points' distances to the mesh surface of the southern side of the park shed $(\mathrm{m})$ 


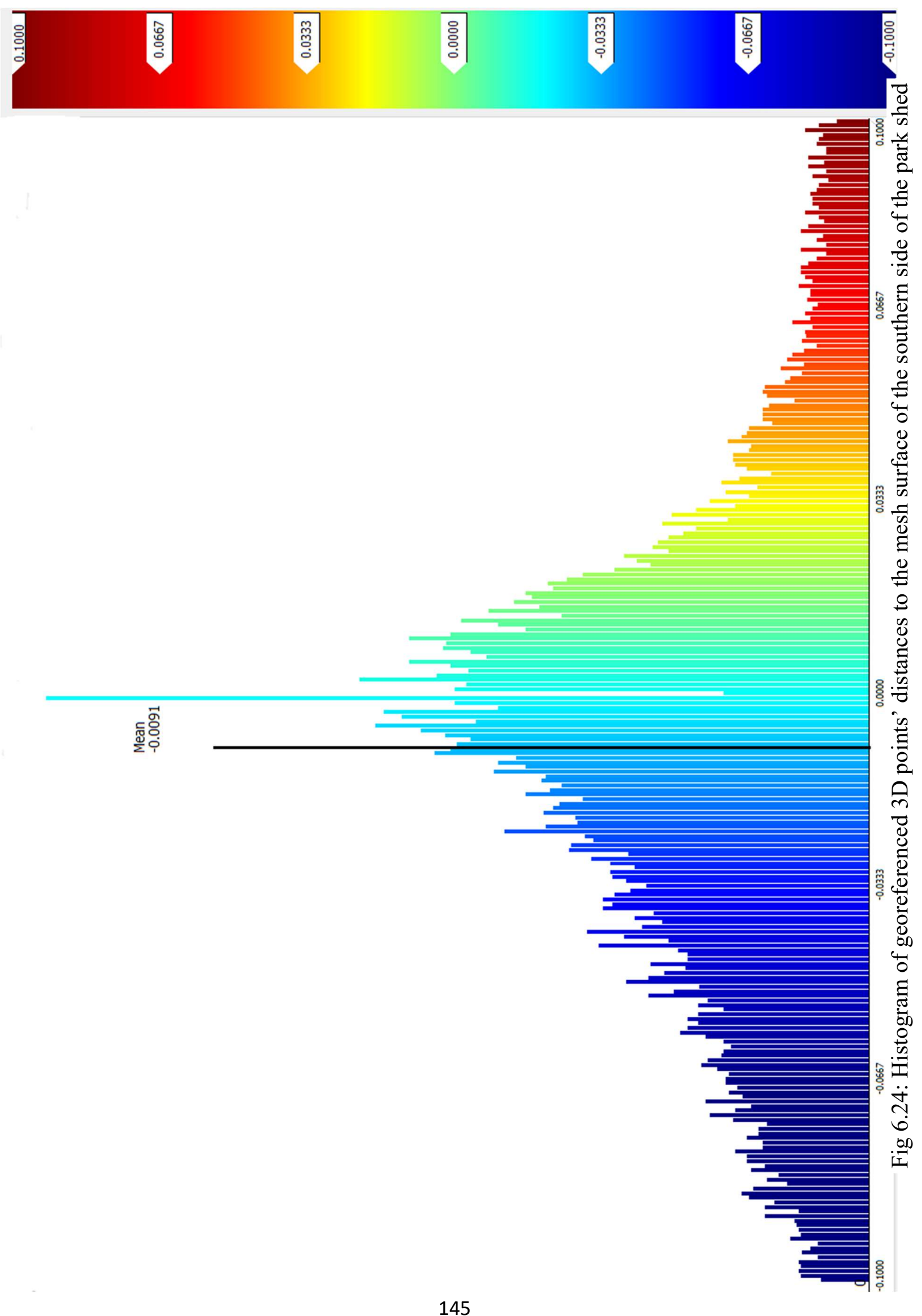


In addition, the northern side of the shed's 3D point cloud has also been assessed. The results of the assessment, which show the 3D points color-coded by the distances to the mesh surface, are presented in Fig. 6.25, while the histogram is shown in Fig. 6.26. The standard deviation has been found to be slightly better, with a value of $3 \mathrm{~cm}$.

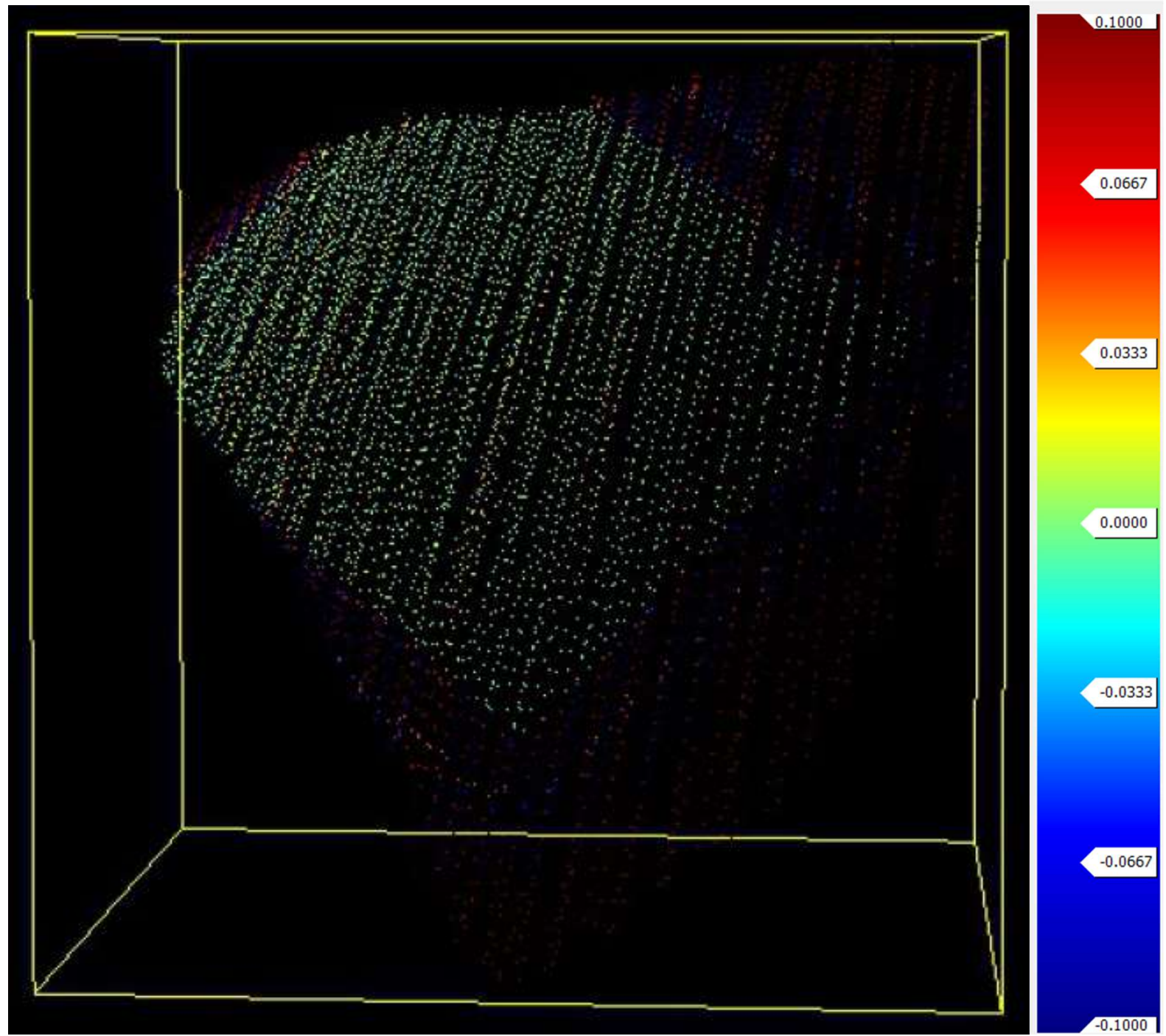

Fig 6.25: Georeferenced 3D points' distances to the mesh surface of the northern side of the park shed $(\mathrm{m})$ 


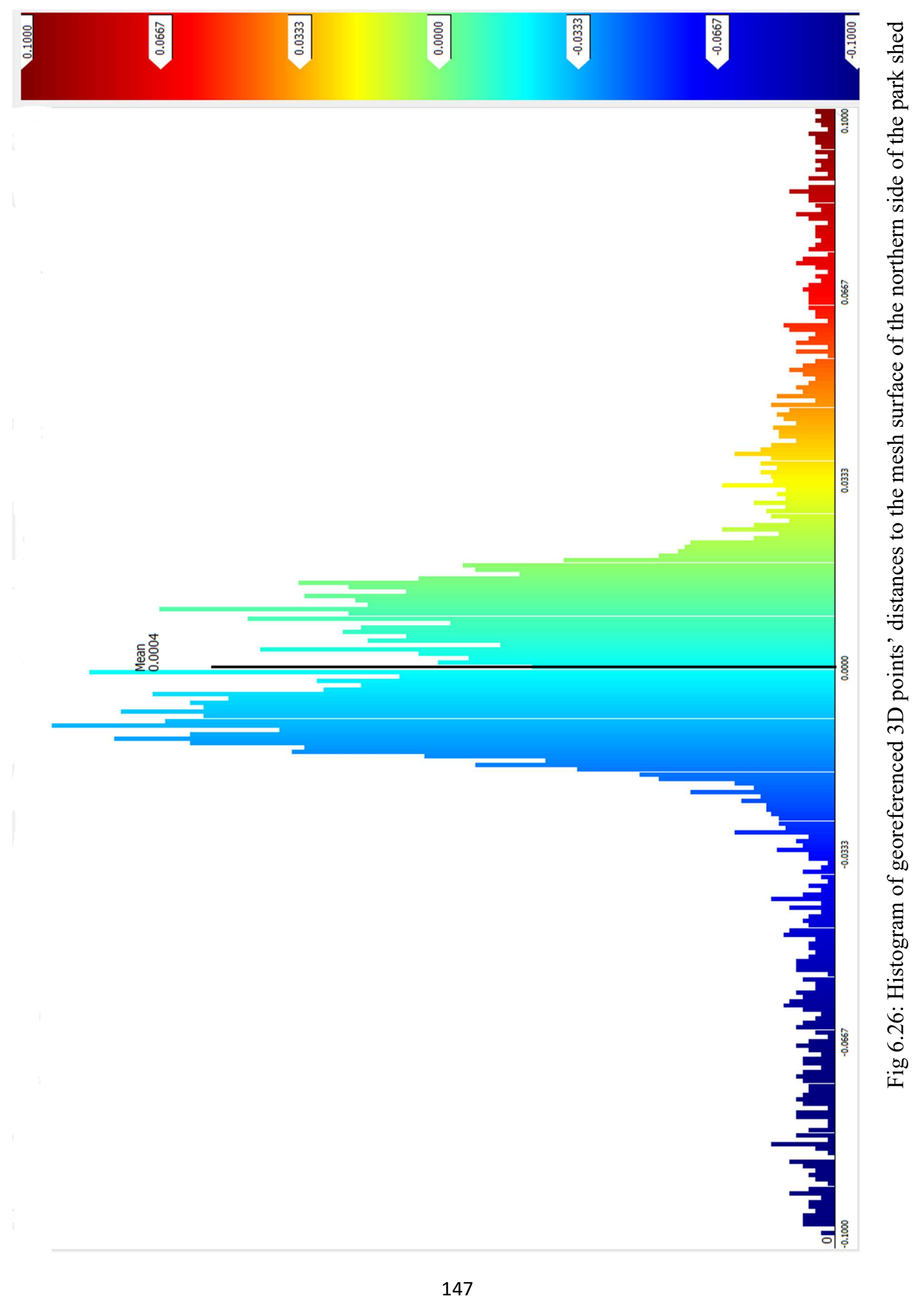


A part of the ground has been assessed as well, with the results of the assessment showing the 3D points color-coded by the distances to the mesh surface depicted in Fig. 6.27; the histogram is shown in Fig. 6.28. The standard deviation was found to be $4 \mathrm{~cm}$, which is the same value observed for the 3D georeferenced point cloud of the southern shed, again highlighting the system's successful mapping of different materials and shapes with precision.

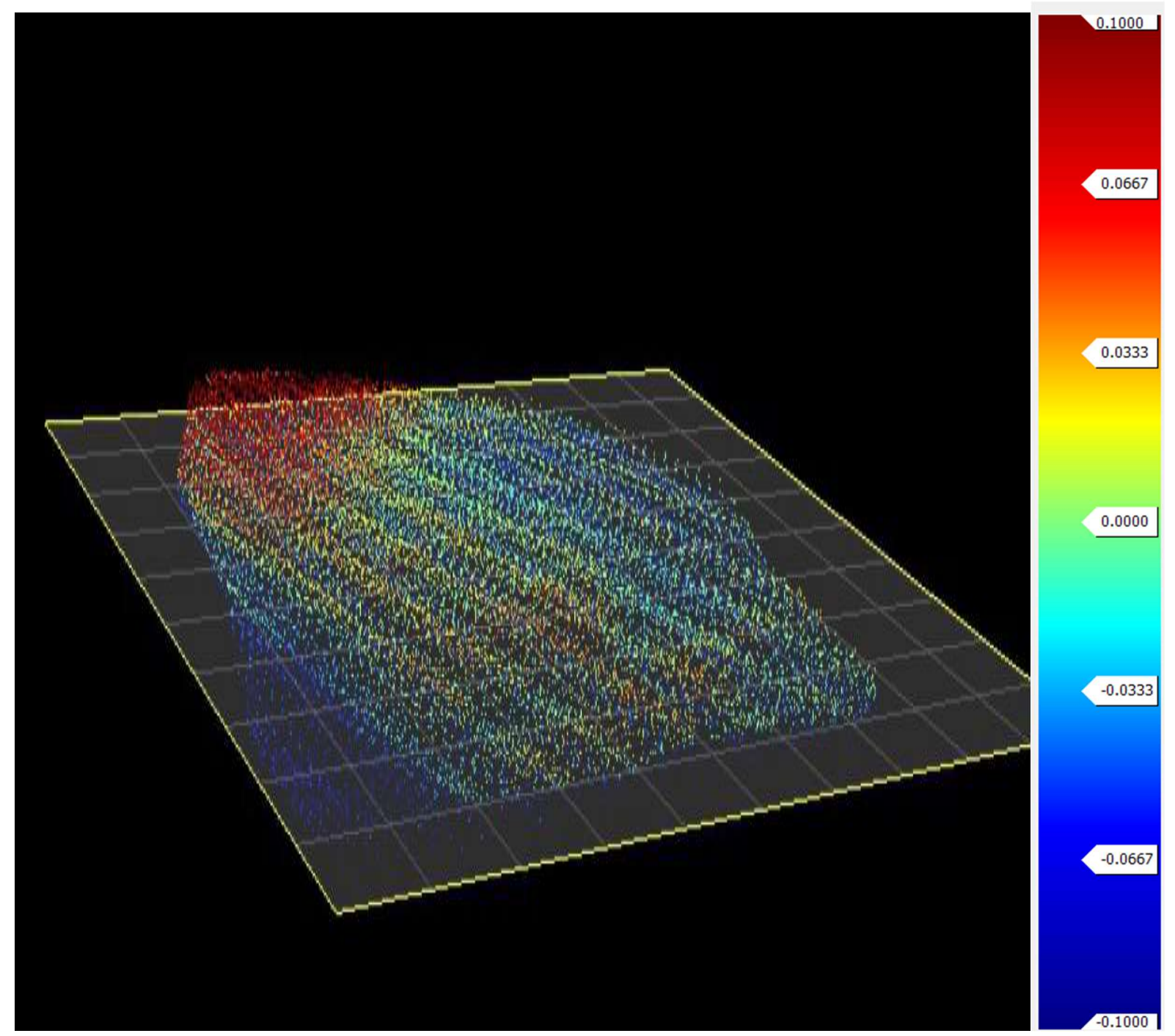

Fig 6.27: Distances to the mesh surface from ground georeferenced 3D points (m) 

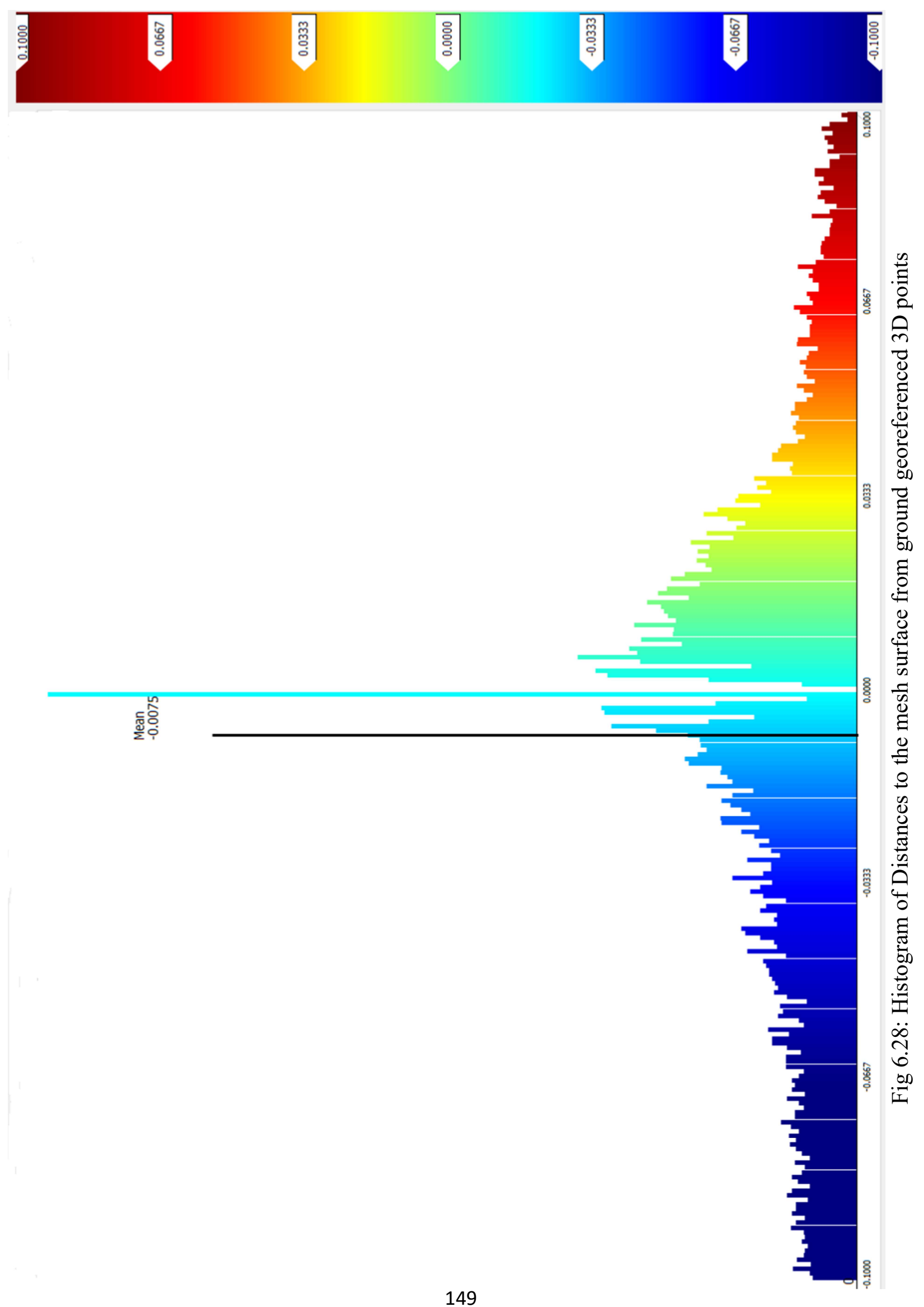
Correspondingly, Polaris data collected in STLS mode were subject to the same criteria of fitting a plane using RANSAC. The 3D point cloud was part of the northern side of the shed. In the process, a mesh surface was created and the normal distances between the points and the mesh surface were calculated. Likewise, the same procedure was applied to a sample of 3D point cloud representing the ground area. A sample of the original readings from Polaris is depicted in Fig. 6.29 , while the results of the distances of the $3 \mathrm{D}$ points to the mesh surface for the northern part and the corresponding histogram are shown in Figs. 6.30 and 6.31, respectively. Additionally, the sample of the 3D point cloud representing the ground area results and calculated histogram are shown in Figs. 6.32 and 6.33, respectively.

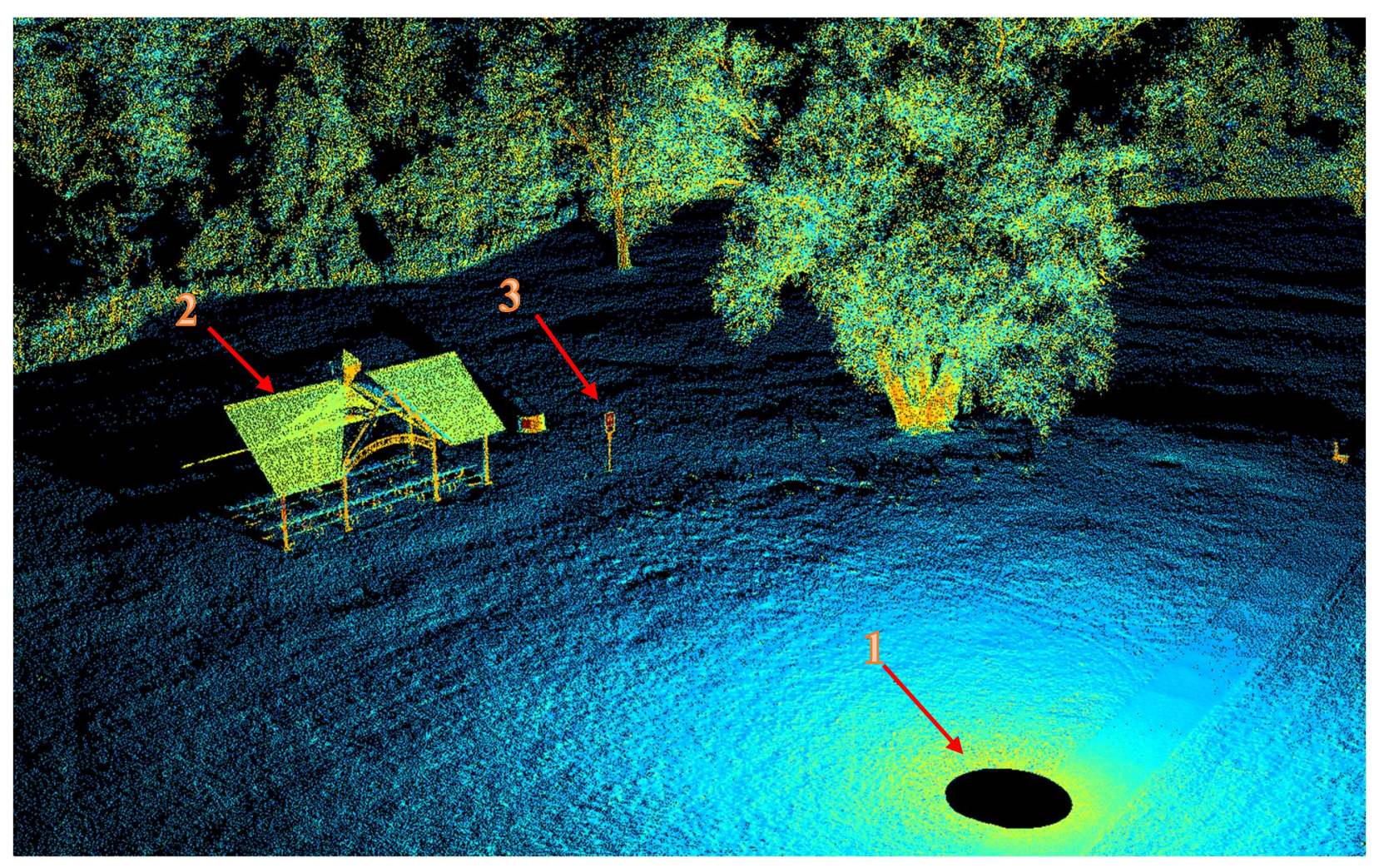

Fig 6.29: Sample 3D point cloud from Polaris

1) Polaris Occupying station 2) Shed with higher level of details 3) A sign 


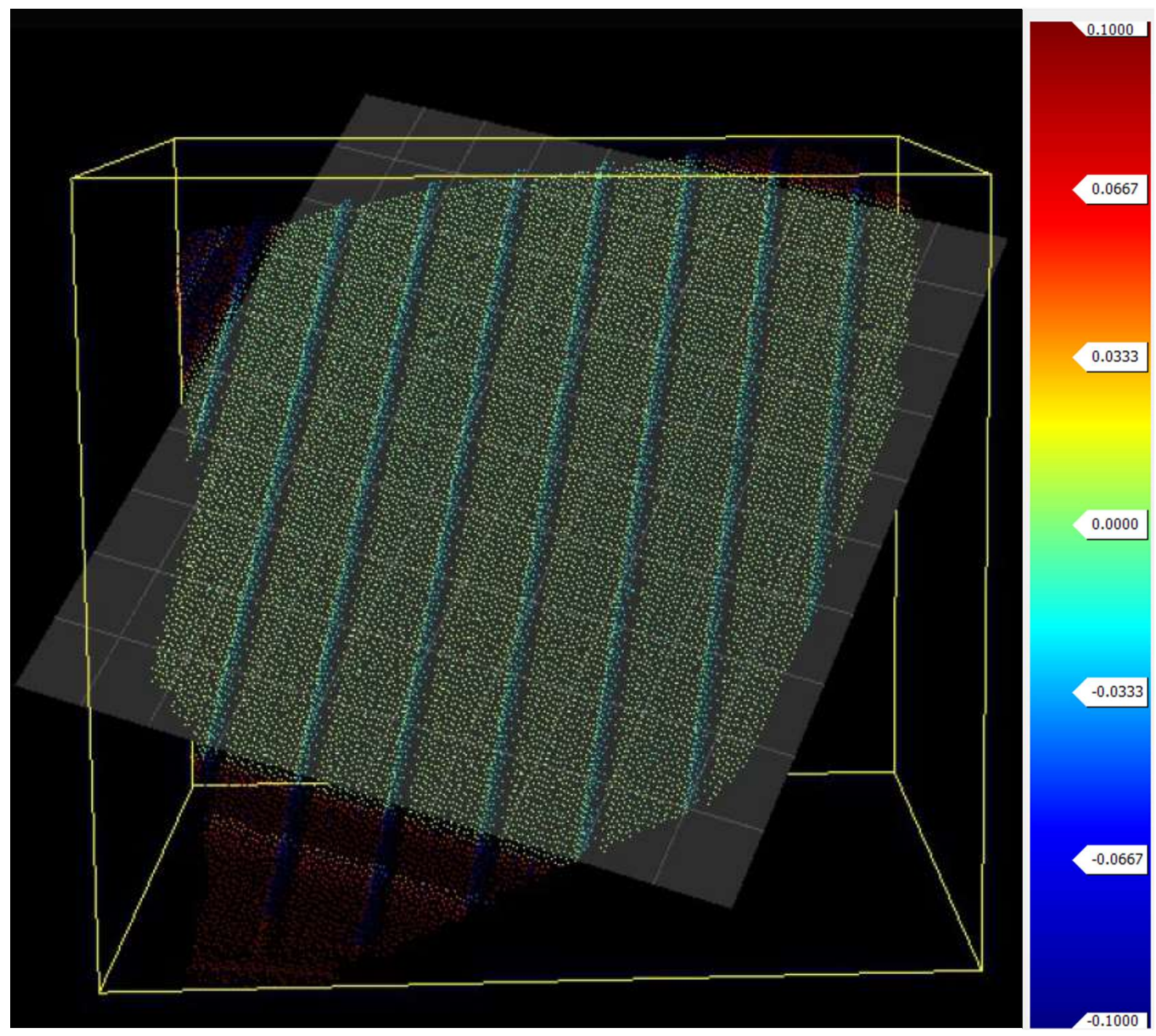

Fig 6.30: Georeferenced 3D points' distances to the mesh surface of the northern side of the park shed (m) Polaris 


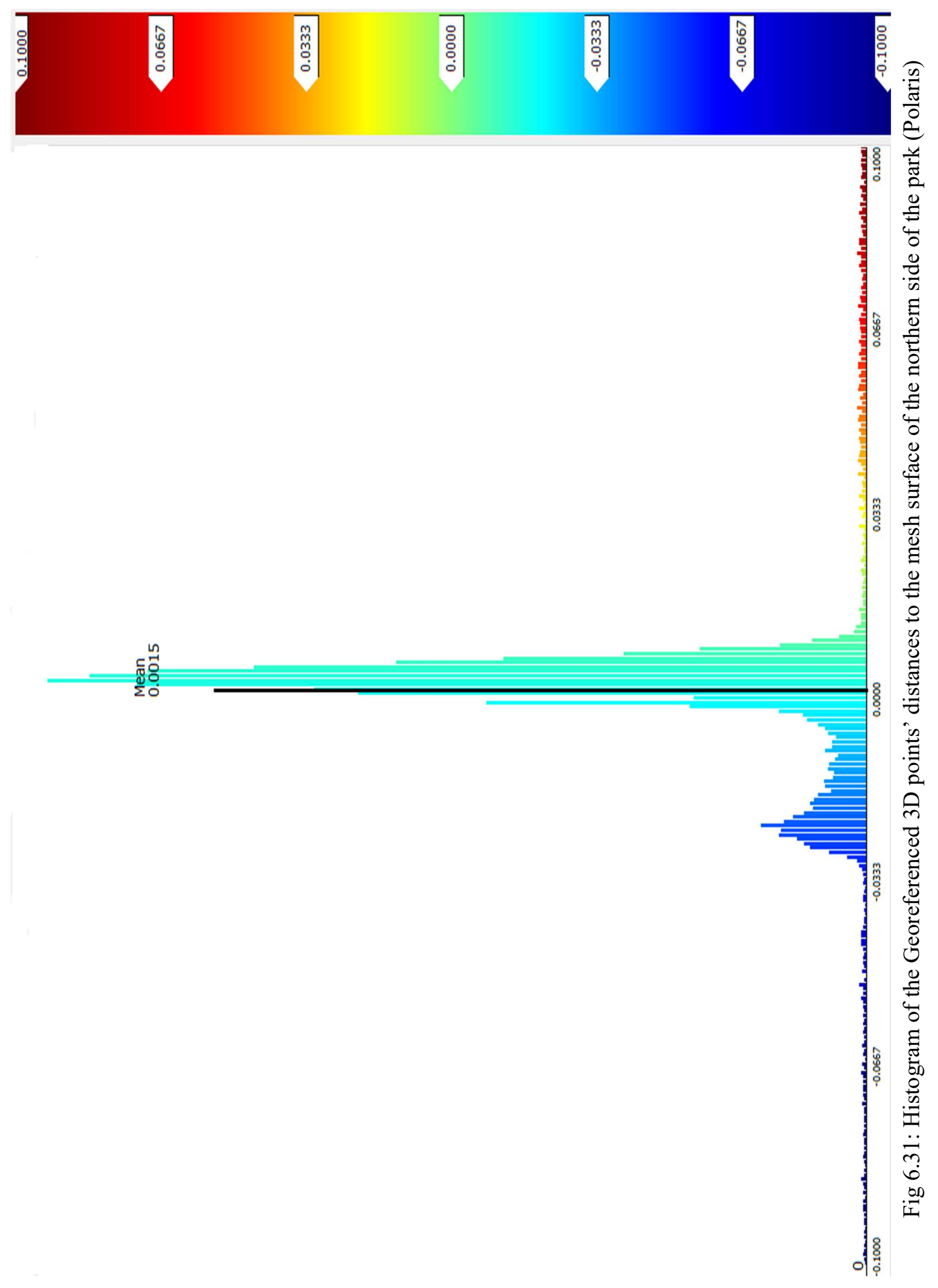


By examining Fig. 6.30, the level of detail that can be mapped by the high-end Polaris scanner can be seen, in that the corrugated pattern of the shed is correctly mapped. The corrugated pattern is represented by the two peaks in the histogram in Fig. 6.31. The standard deviation has been found to be $2 \mathrm{~cm}$. It is worth highlighting that the accuracy of the Polaris system is $5 \mathrm{~mm}$, as per the manufacturer's provided specifications sheet. The corrugated pattern may explain the standard deviation of the $2 \mathrm{~cm}$ observed.

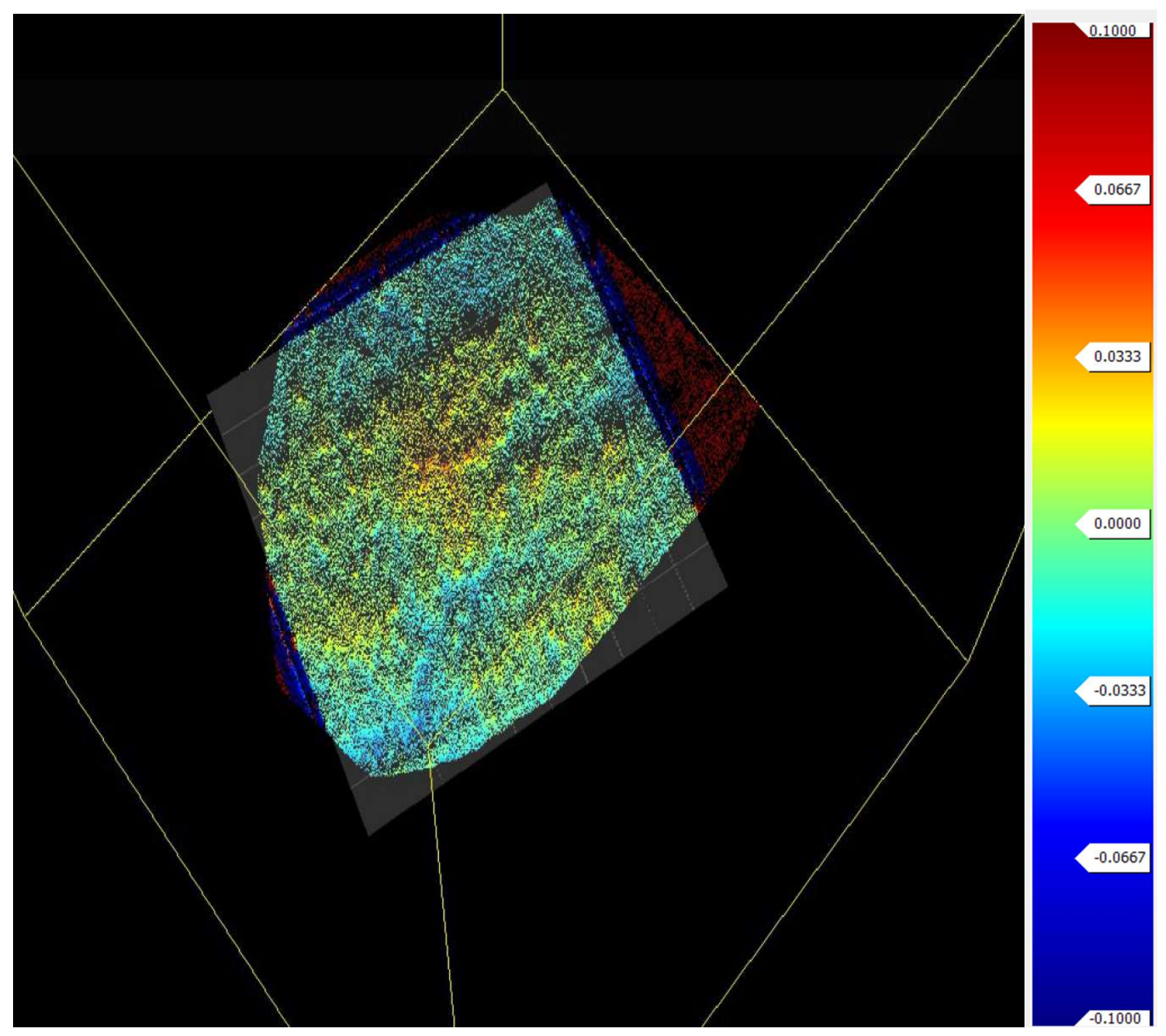

Fig 6.32: Distances to the mesh surface from ground georeferenced 3D points (Polaris) 

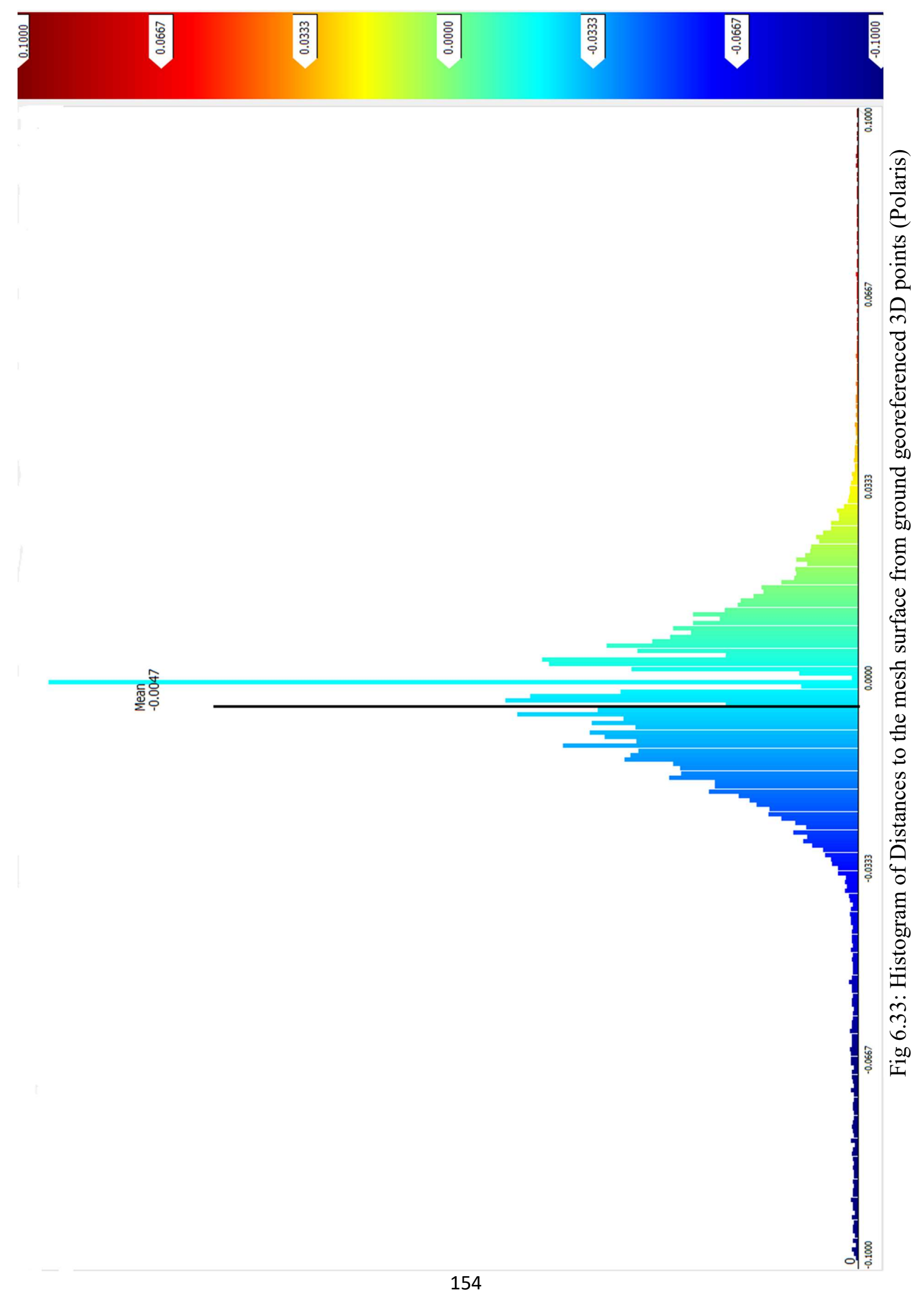
As shown in Fig. 6.32, Polaris captured a high level of detail in the ground surface pattern, as there is variation in the $3 \mathrm{D}$ point distances to the mesh surface. This is due to the nature of the vegetation cover in the area. Figure 6.33 shows the histogram representing the distances calculated. As can be seen in the figure, it follows the shape of a normal distribution curve with a mean value almost equal to zero. The standard deviation was found to be $2 \mathrm{~cm}$. Note that the Polaris's observed precision of $2 \mathrm{~cm}$ is much less than the $5 \mathrm{~mm}$ listed in the specifications, but this may be attributed to the corrugated shape of the shed and the nature of the vegetative cover in the ground area. The accuracy of the $3 \mathrm{D}$ point distance to the mesh surface from the developed system has been found to be in the range of 3-4 cm, which is very good compared to the $2 \mathrm{~cm}$ deviation observed using the high-end Polaris system. Nevertheless, the 3-4 cm range utilizing the developed system could be attributed to the precision of the system itself, whereas the variation in the Polaris case would likely be due to the nature of the surfaces mapped, such as the detailed corrugated pattern correctly mapped in the shed area and shown in Fig. 6.30.

\subsection{Captured Data Analysis (SfM)}

The captured imagery from the DJI Phantom 3 drone shows that, according to the pre-flight trajectory programmed, the drone autopilot followed the path entered. The pre-defined flying path for the autopilot maintained a 70\% front lap and 50\% side lap. The larger the front lap and side lap, the greater the ability of the SfM workflow to produce reliable results, as it increases the keypoints matched between the different overlapping imagery. Greater precision of the 3D geometry of the area produced from the SfM workflow is related to an increased number of matched keypoints that appear in more than two images. Therefore, maintaining the front and side laps is crucial for the SfM workflow to operate reliably, so the usage of autopilot with a pre-programmed flight trajectory is essential.

The overlapped captured images have been used to produce the 3D geometry of the mapped area through a Pix4D environment. The presence of the geotagged information in the images EXIF aided in optimizing the 3D data-making. The steps used for building the 3D map from the overlapping imagery can be summarised as follows:

1- Reading the EXIF data to know roughly the image sequences and positions

2- Computing key-points in each image that are easily identifiable

3- Computing matches between the key-points

4- Calibrating the IO,EO camera parameters

5- Optimizing (recursive with steps 6,7 where uncertainties are calculated)

6- Optimizing with the geo information

7- Computing rematches

8- Dense-matching 
The area mapped by the DJI phantom 3 drone covers 7.5 acres and the average GSD is $2 \mathrm{~cm}$. The resultant point cloud and the positions of the camera is shown in Fig 6.34.

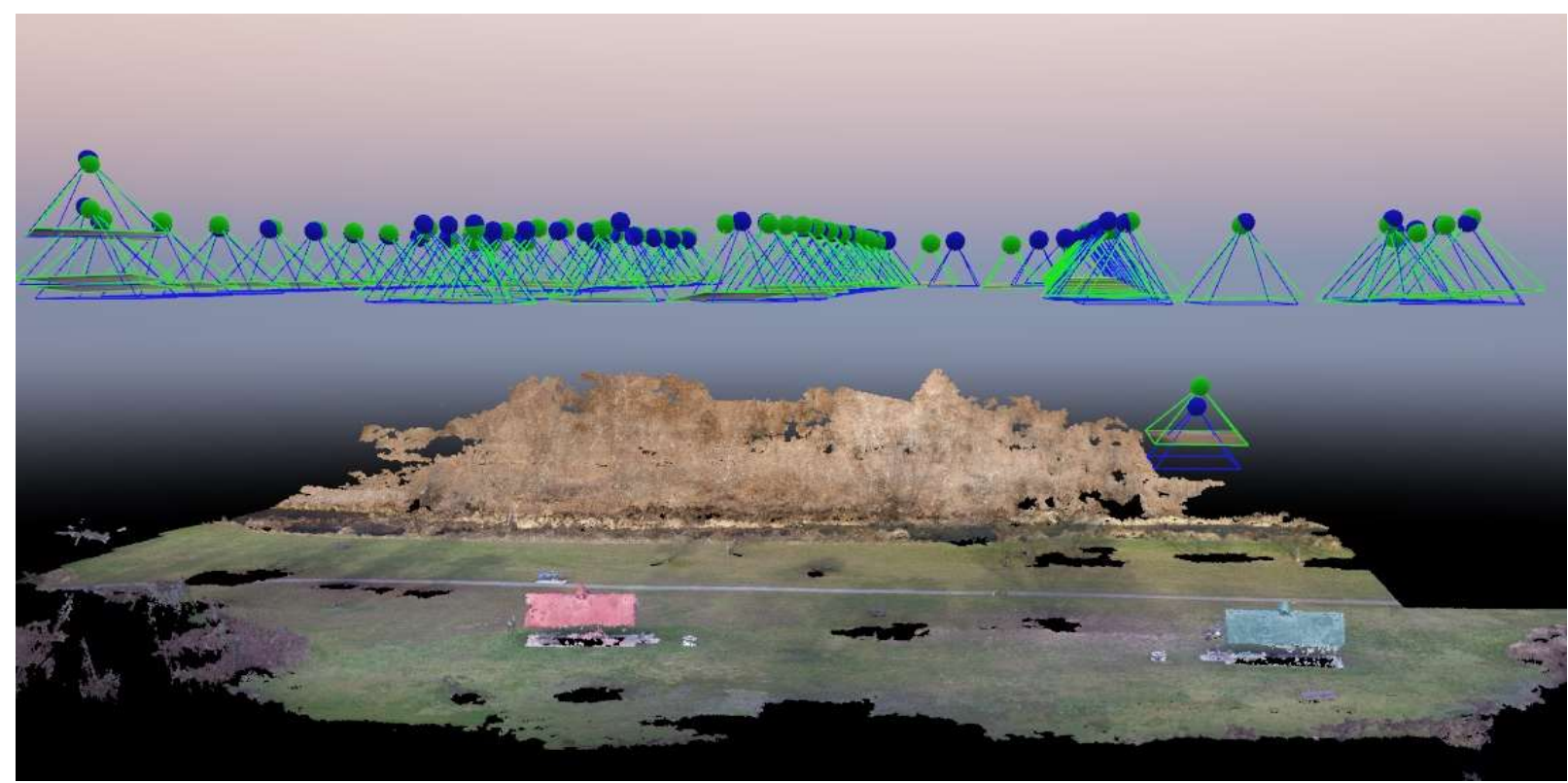

Fig 6.34: Camera positions and the resultant point cloud

As seen in the figure, the general layout and the features and colors in the area are still adequate. However, there are missing areas that are obviously problematic for the workflow to resolve. These missing areas can be attributed to poor texture conditions and the presence of trees. For example, the two trees that are clearly mapped and shown in Fig. 6.22 are missing in the produced 3D point cloud from SfM. An example of the problematic areas for the SfM is illustrated in Fig. 6.35.
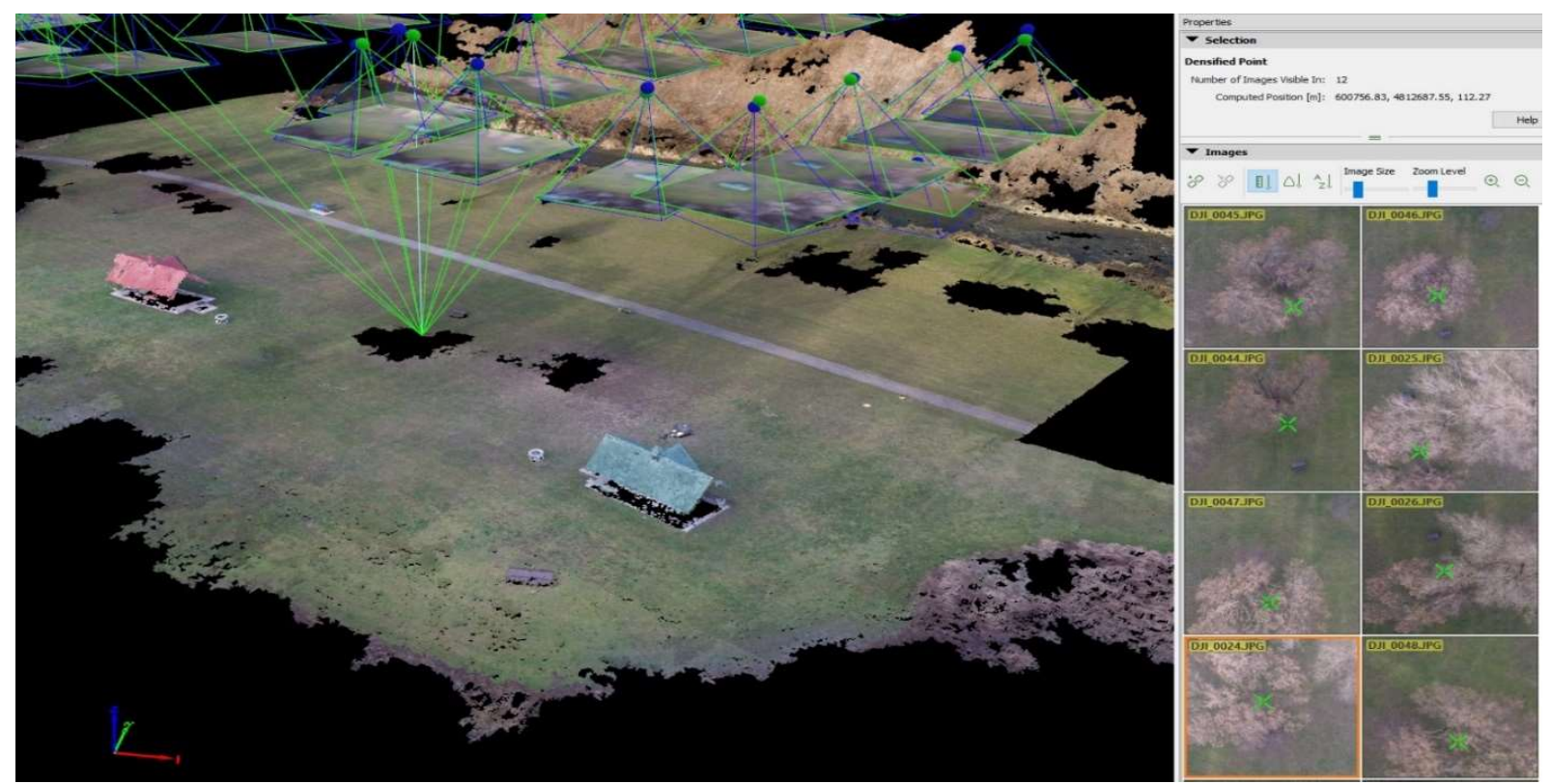

Fig 6.35: Missing area in the SfM output 
As shown, ray tracing is used to find the points in the different images that are used in the SfM processing. Again, it is obvious that the problematic areas are those covered by trees, as can be seen in the right-hand side of the figure. The resultant 3D point cloud from the SfM has been superimposed on top of an orthophoto covering the mapped area. This is depicted in Fig. 6.36.

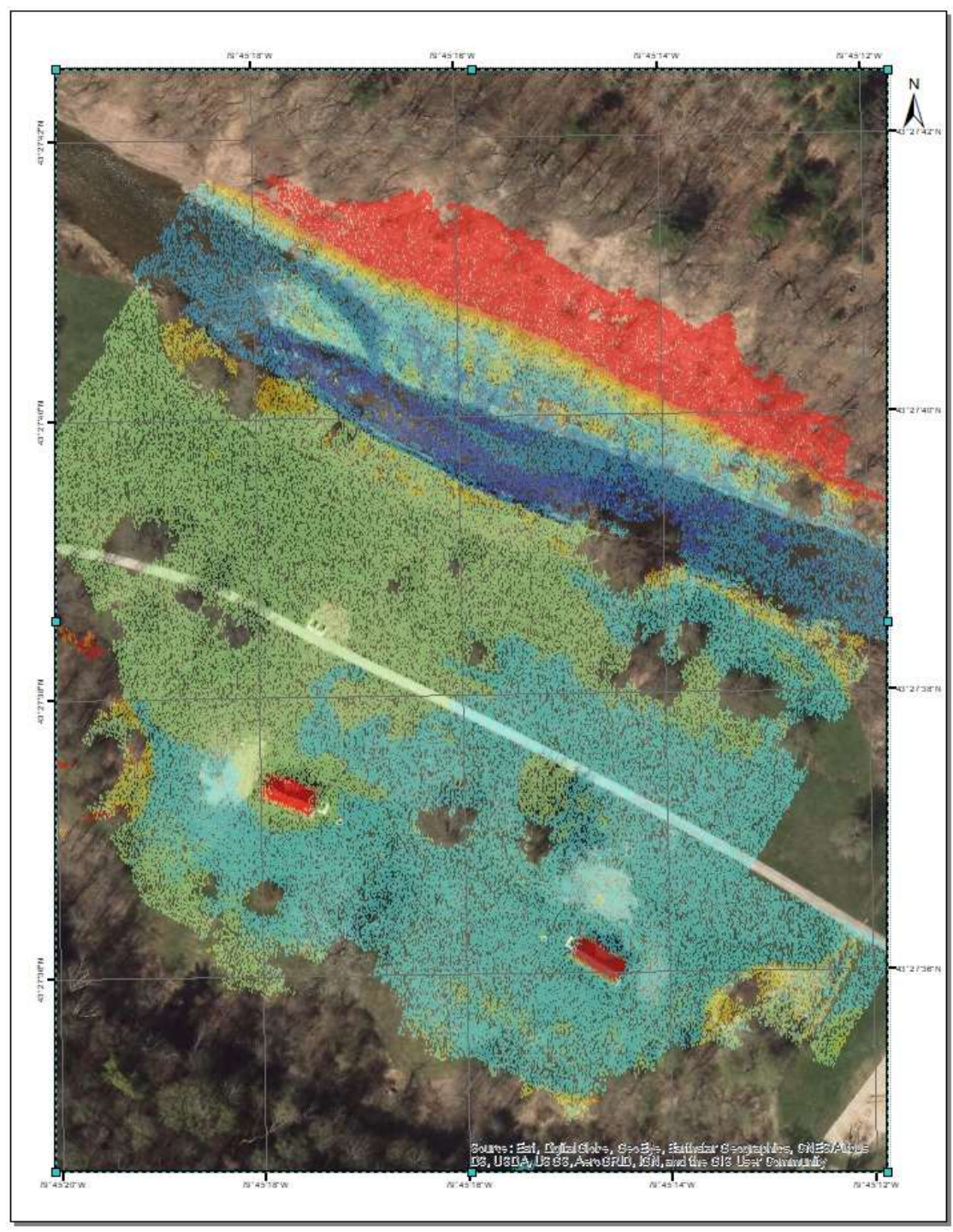

Fig 6.36: 3D point cloud from the SfM workflow

(Color coded with elevation; lowest-blue; highest-red)

Figure 6.36 illustrates how the SfM workflow successfully captured the different elevation of the features in the area. The high elevation red colored features represent the sheds and the increasing sloped area at the top of the image. Nevertheless, the areas covered by trees are again problematic and are missing, as Fig. 6.25 clearly depicts. This highlights the dependability of the SfM workflow on the features being mapped and the quality of the overlap and key matches between the images. It still, however, shows very good results, correctly portraying the difference in elevation between features. Moreover, it correctly depicts the water area and the walking path. These areas proved 
problematic for the LiDAR- based sensor, due to the nature of the LiDAR data operating in the NIR, which is almost completely absorbable by water. Also, the SfM output features the natural colors and textures of objects, which is advantageous in visual interpretation-related tasks. Note that the SfM depends heavily on the matching between key-points and the number of images these matches are present.

The quality-check parameters for the SfM model are presented in Tables 6.5, while the adjustment results for the bundle block adjustment optimization are presented in Table 6.6. Table 6.7 highlights the number of 3D points calculated from 2D key-point matches. The values provided in the three tables do not indicate any alarming values as defined by Pix4D processing environment, suggesting an adequate resultant model. The average Automatic Tie Point (ATP) pixel reprojection error magnitude and direction are visualized in Fig. 6.37.

Table 6.5: SfM Quality check

\begin{tabular}{|l|l|}
\hline Images & 62 out of 74 images calibrated $(83 \%)$, \\
\hline Dataset & $\begin{array}{l}\text { median of } 8236.65 \text { matches per calibrated } \\
\text { image }\end{array}$ \\
\hline Camera Optimization & $\begin{array}{l}2.63 \% \text { relative difference between initial and } \\
\text { optimized internal camera parameters }\end{array}$ \\
\hline Matching & median of 40425 keypoints per image \\
\hline
\end{tabular}

Table 6.6: Bundle block adjustment results

\begin{tabular}{|l|l|}
\hline $\begin{array}{l}\text { Number of 2D Key point Observations for } \\
\text { Bundle Block Adjustment }\end{array}$ & 498126 \\
\hline $\begin{array}{l}\text { Number of 3D Points for Bundle Block } \\
\text { Adjustment }\end{array}$ & 208501 \\
\hline Mean Re-projection Error [pixels] & 0.223 \\
\hline
\end{tabular}

Table 6.7: 3D points calculated from 2D key points matches

\begin{tabular}{|c|c|c|}
\hline \multicolumn{3}{|c|}{ Number of 3D Points Observed } \\
\hline In 2 Images 160048 & In 11 Images 94 & In 21 Images 7 \\
\hline In 3 Images 31408 & In 12 Images 50 & In 22 Images 5 \\
\hline In 4 Images 9676 & In 13 Images 23 & In 23 Images 6 \\
\hline In 5 Images 3607 & In 14 Images 3 & In 24 Images 3 \\
\hline In 6 Images 1647 & In 15 Images 3 & In 25 Images 3 \\
\hline In 7 Images 949 & In 16 Images 2 & In 26 Images 4 \\
\hline In 8 Images 567 & In 17 Images 20 & In 27 Images 2 \\
\hline In 9 Images 286 & In 18 Images 19 & In 28 Images 1 \\
\hline In 10 Images 138 & In 19 Images 12 & In 30 Images 1 \\
\hline & In 20 Images 10 & In 32 Images 1 \\
\hline
\end{tabular}




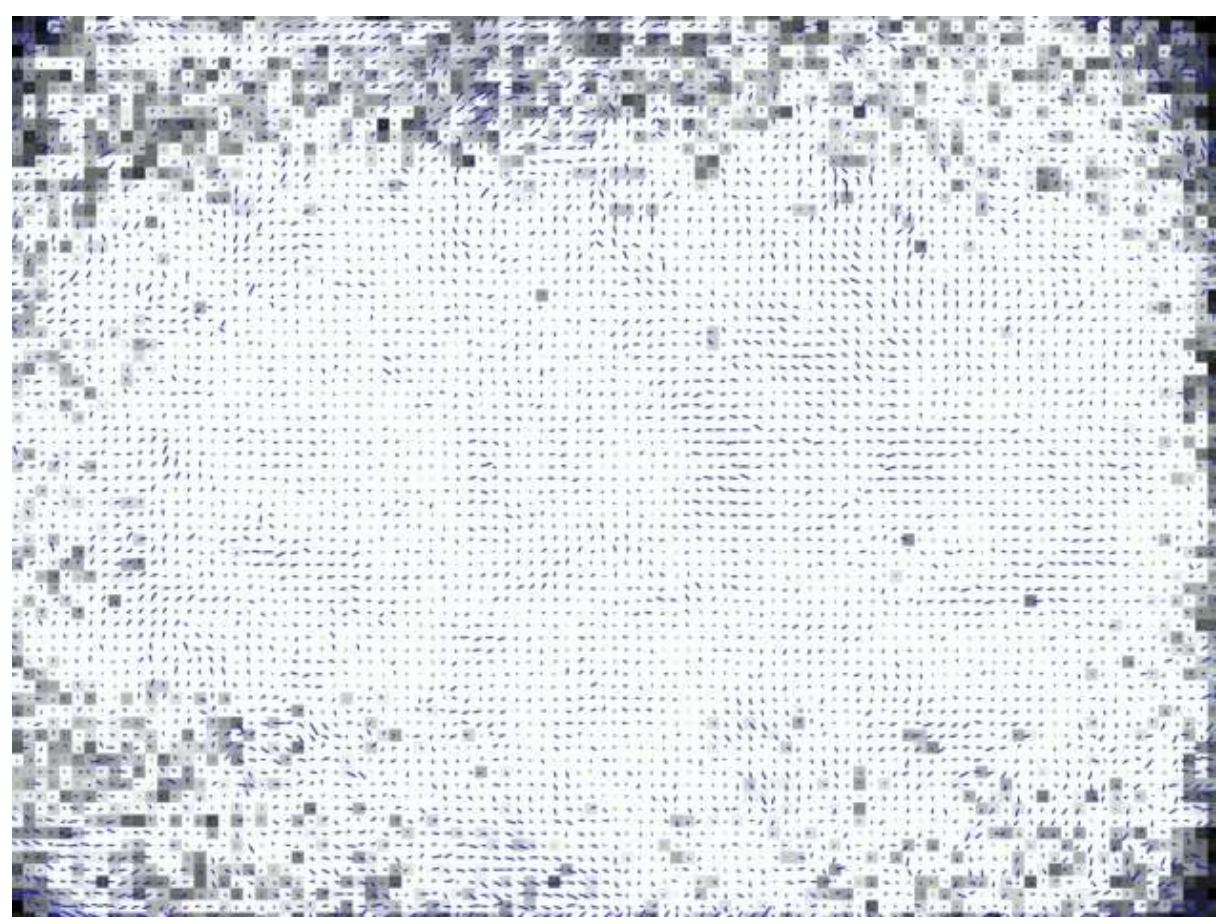

Fig 6.37: Average automatic tie points ATP

Fig 6.37 shows the number of ATPs per pixel, averaged over all images of the camera model. The image is color coded between black and white. White indicates that, on average, more than 16 ATPs have been extracted at the pixel location while black indicates that, on average, 0 ATPs have been extracted. The larger the dark portion indicates less ATP and a less optimal 3D calibrated model which is associated with sub-optimal imagery conditions. As it is shown with the SfM model in airborne mode, the geolocation information through the imagery EXIF geotags aids in the $3 \mathrm{D}$ reconstruction of the environment. The strength of the ATP matches and the camera positing uncertainty is highlighted in Fig 6.38. 


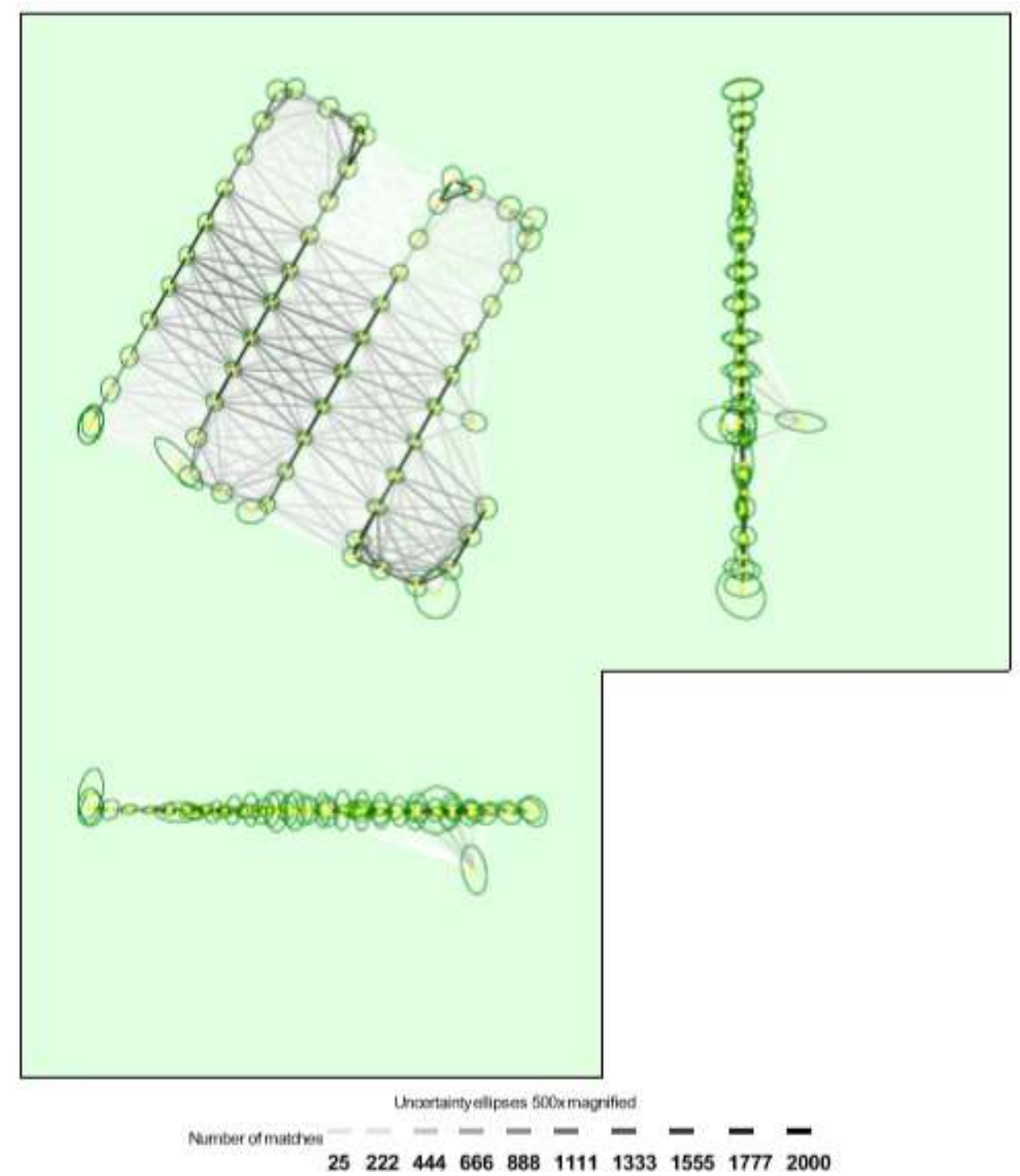

Fig 6.38: 2D key points matches

Fig 6.38 demonstrates that the darker the link, the more point matches are present. The strength of the matches is indicated by the images through the number of points matched. In addition, the dark green ellipses indicate the relative camera position uncertainty of the bundle block adjustment. The light color links represent the need for manual tie point additions, which would be problematic in feature-less or poor texture areas. Simply acquiring more images (i.e., adding more information) is not guaranteed to solve the challenge. Furthermore, the inversely proportional relation between the number of matches and the uncertainty of the camera position solution from the SfM workflow is also depicted in the figure, emphasizing the dependency of the SfM resultant model on the keypoint matches between the images.

The dependency of the resultant point cloud on the key-point matches between the images reemphasizes one of the disadvantages of SfM, and unless the scale is constrained by GNSS or a known measured distance, the output is up to scale. Nevertheless, it is still a cheap alternative and has a texture and true color visualization which appeals to a wide range of end-users. Conversely, 
the 3D geometry is directly captured by the usage of the new LiDAR-based developed system, but lacks texture and color information and performs sub-optimally in wet areas or over water bodies, as has been highlighted in the data captured. Thus, to overcome the drawbacks of each approach, the fusion between them is spontaneous.

\subsection{DSM Generation:}

To test the generation of the DSM from each data modality, the resultant 3D point cloud captured by the LiDAR-based developed system at hand and the 3D point cloud built through the SfM workflow was used to generate a DSM for the mapped area. The conversion from the 3D representation of the mapped area to a 2.5D representation of DSM followed the same unified processing steps for the data modality of the LiDAR-based and optical imagery through SfM. The 3D data represented in las format has been projected to a raster format through applying a mean filter to the data. Further, the data from both the LiDAR-based mapping system and the SfM optical imagery has been masked with the exact same area boundary to unify the area covered by each.

A binning approach has been applied to the data to transform the 3D point cloud in the las format to a raster-based format. The spatial resolution of the output raster data has been unified and generalized for both data type sources to $20 \mathrm{~cm}$ to account for any misalignment or artifact that may be present within the data. The same interpolation method (IDW and natural neighbor) has been applied to fill in the areas with missing data, such as areas covered by trees (as was shown in the SfM application) or wet and shadowed areas (as was shown in the LiDAR-based mapping system utilization). The generated DSM from the data captured by the LiDAR-based mapping system is presented in Fig. 6.39 and the DSM generated from the data resulting from the application of the SfM workflow on the DJI Phantom 3 Professional optical imagery is given in Fig. 6.40. 


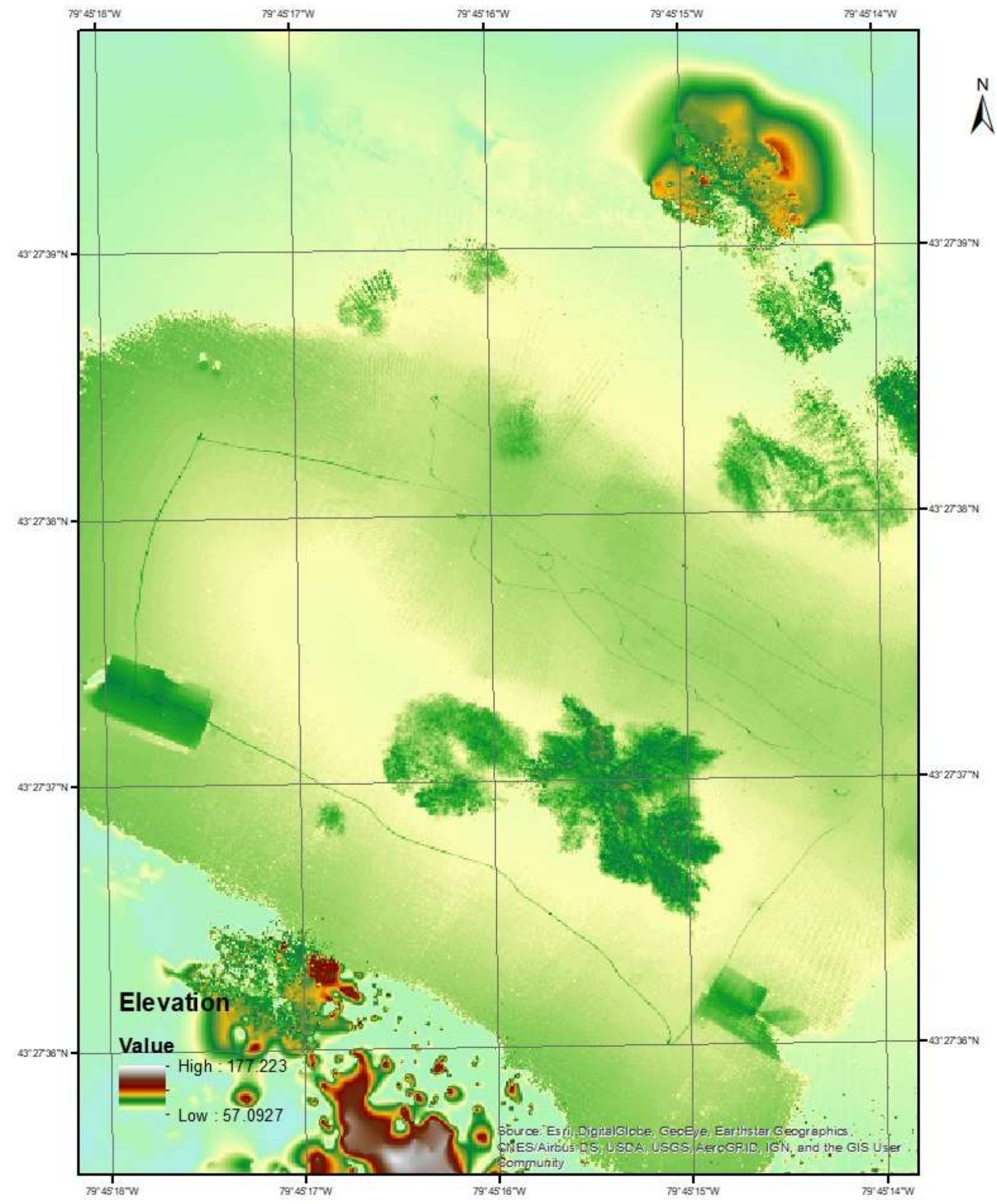

Fig 6.39: LiDAR-based DSM 


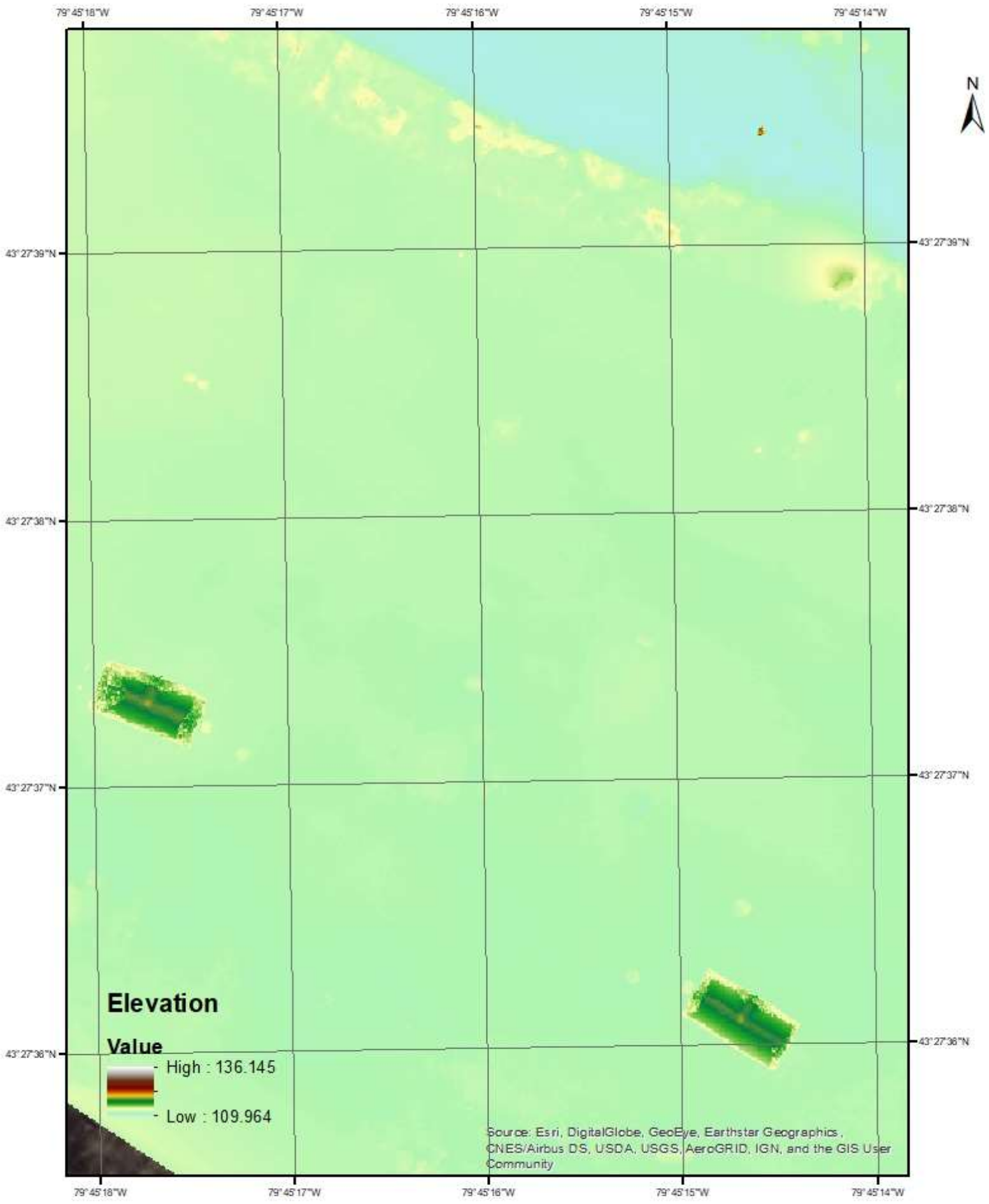

Fig 6.40: SfM-based DSM 
By contrasting Figs. 6.39 and 6.40, which represent the LiDAR-based and SfM DSM, respectively, we can see the missing areas from the SfM approach caused by the presence of trees, as well as the problematic wet and water areas from the LiDAR-based data. For a better interpretation, the DSM from the LiDAR-based mapping system and the DSM from the SfM workflow are shown in Figs. 6.41 and 6.42, respectively, overlaid on an orthophoto of the area.

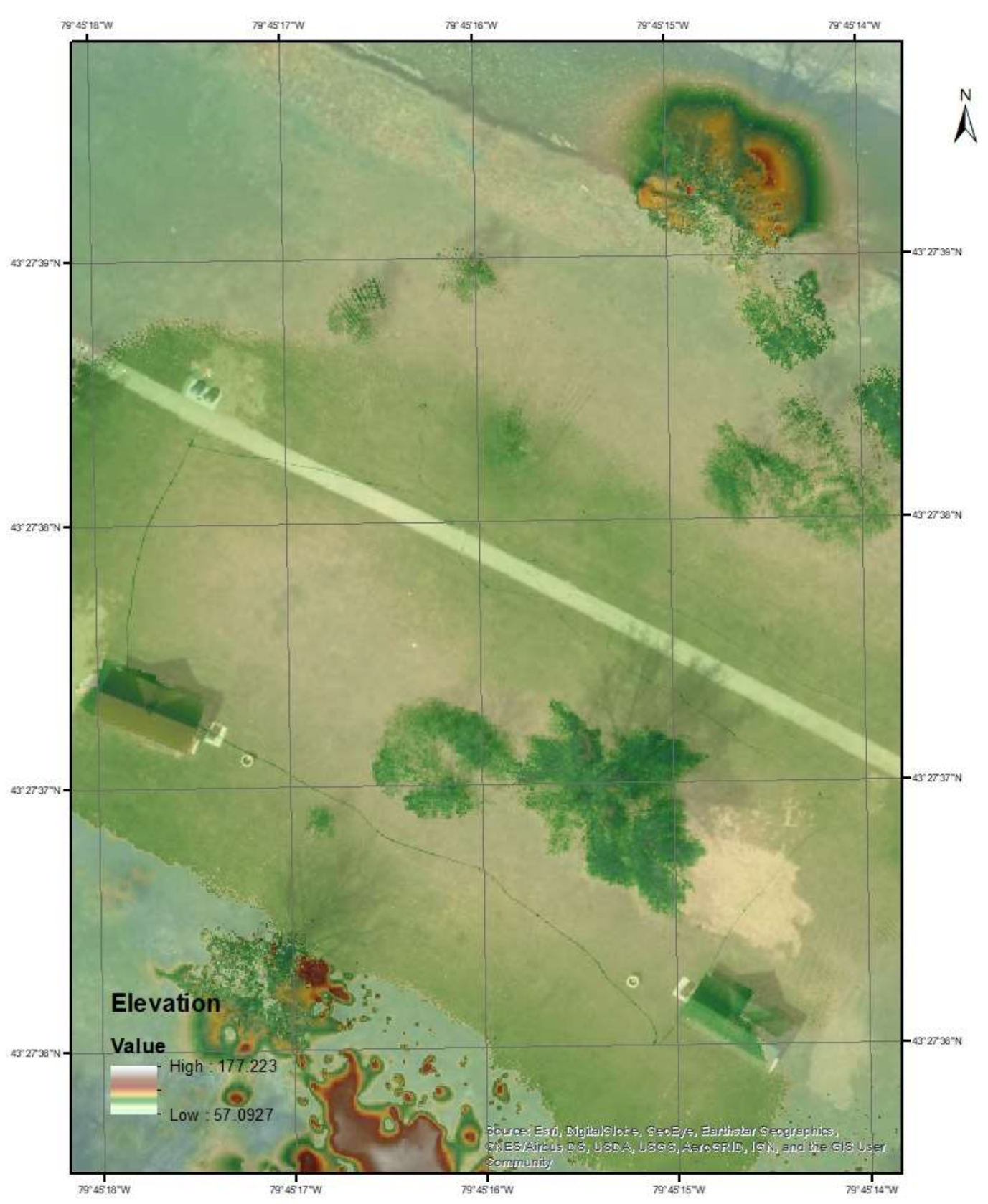

Fig 6.41: LiDAR-based DSM overlaid over Orthophoto 


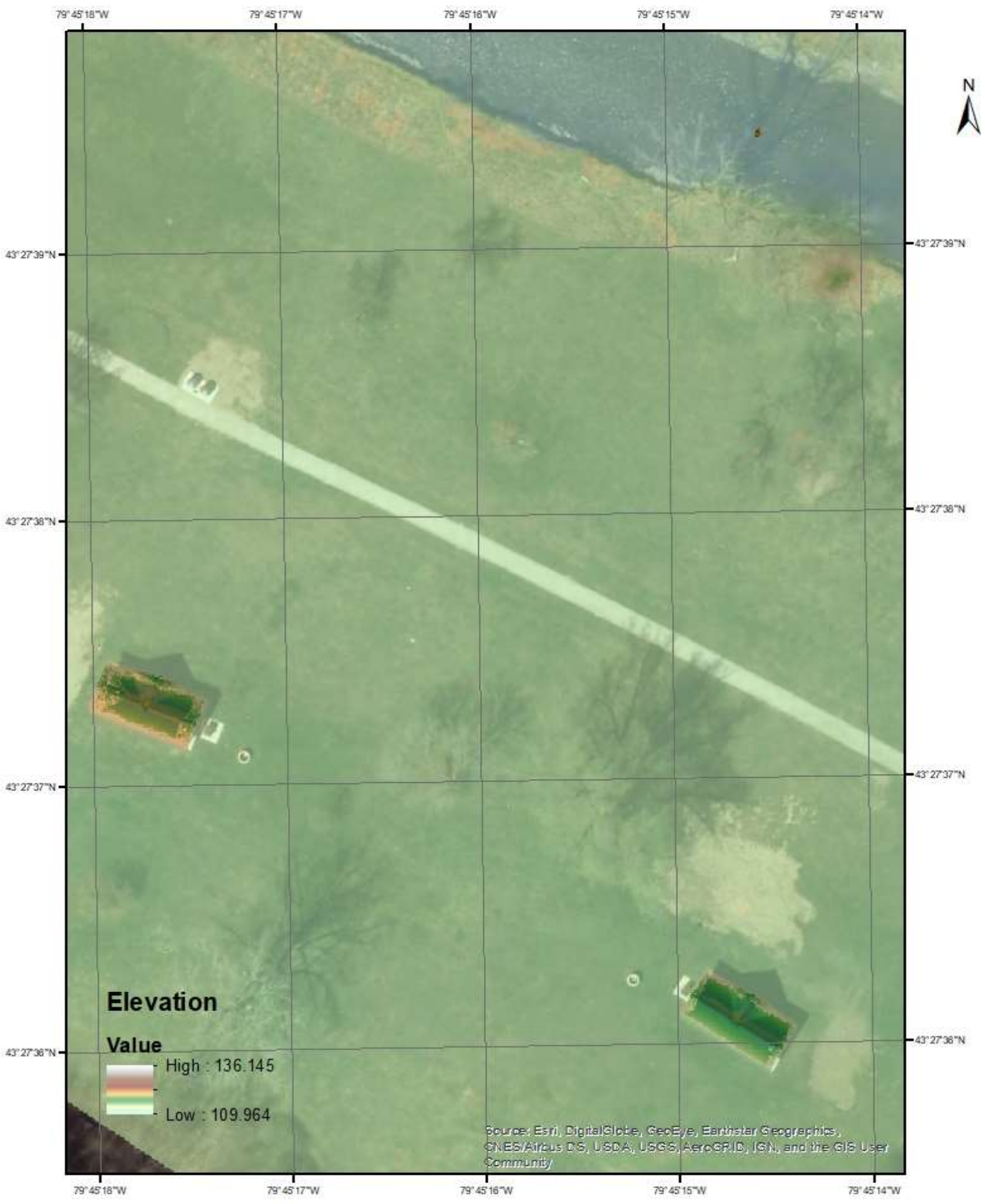

Fig 6.42: SfM-based DSM overlaid over Orthophoto 
Quantitative assessment of the DSM generated from different data capturing mechanisms is challenging. A DSM from a Polaris point cloud that covers the mapped area has been created. The nature of producing the DSM and the georeferencing process is quite dissimilar to the LiDARbased developed mapping system, the SfM 3D output built from matching key-points in optical imagery, and the Polaris dataset that has been captured with a terrestrial point of view. The misalignment between the different datasets makes a simple subtraction between the pairs of DSM error-prone. In order to neutralize any mismatching between the cell-by-cell positions from the three datasets and to compensate for any misalignment between features, the relative height differences between the same feature pairs on each dataset were compared independently. For example, using the SfM DSM, for different random cells on each surface the height difference between the shed surface and the ground surface was measured and compared to the height difference between the shed surface and the ground surface in the Polaris dataset, which has been deemed as the ground truth. Likewise, using the LiDAR-based USL DSM, for different random cells on each surface, the height difference between the shed surface and the ground surface was measured and compared to the height difference between the shed surface and the ground surface in the Polaris dataset. It is worth noting that, typically in most of the applications (especially for hydrology), height differences are more important than absolute elevation. The same applies for line-of-sight-related applications.

Regarding differences between the highest and lowest points on the western shed and their difference to the ground level, the Polaris dataset and the LiDAR based ULS developed system showed good agreement in the shed height and the distance to the ground level. The agreement is in the few decimeters range. The SfM showed slightly worse values.

The presence of GCPs and sufficient features in the area mapped might have provided a better mean for the quantitative assessment task, especially for the tree-covered areas due to the different viewing points of the ULS, SfM and Polaris. As noted, the ULS, SfM has an aerial viewing point, while the Polaris has a terrestrial viewing point. Also, as stated in the qualitative assessment, the SfM workflow applied to the mapped area has been consistently problematic for tree-covered areas. Still, the potential gain in augmenting both approaches through their fusion is evident for the completeness and applicability of different environment coverage.

Additionally, the successful deployment of the developed LiDAR-based mapping system and the good agreement of the derived data products from it (assessed qualitatively and quantitatively against the state-of-the-art high-end terrestrial laser scanner, Polaris) proved the applicability and versatility of the developed mapping system. To the best of the author's knowledge, this is the world's first multipurpose LiDAR-based mapping system that is designed and developed to be readily deployed in terrestrial, mobile and UAV modes of operation. The developed mapping system has been successfully integrated on the hardware and software levels and successfully deployed in the three different data acquisition modes, namely, static terrestrial laser scanning, mobile mapping, and UAV laser scanning. The 3D point cloud of the mapped areas in the three different modes of operation showed very good agreement against accurate datasets that had been acquired by expensive, high-end LiDAR equipment. 


\section{SUMMARY, CONCOLUSIONS, KEY CONTRIBUTIONS, AND RECOMMENDATIONS FOR FUTURE RESEARCH}

\subsection{Summary and Conclusions}

The main focus of the research presented was the development of a generic framework for a multipurpose, platform-independent, LiDAR-based mapping system. The developed system uses a direct georeferencing approach in 3D mapping and follows a plug-n-go concept, as it can readily be deployed in three different modes of operation, regardless of the platform used. Although the system uses non-high-end sensors, the qualitative and quantitative assessment of its deployment in the three mapping modes proved its practicality, versatility, and accuracy for the final 3D product. Further, the system's ease of deployment and crosscutting ability in the three mapping modes meets the needs of diverse end-user segments, and the system can easily be deployed in the three modes of data acquisition, namely, stationary, mobile and UAV-based..

\subsubsection{Generic framework of the system}

A generic framework pertaining to system design and development processes was developed. The system design criteria set in the present research was to develop a system that is cost-efficient yet achieves up to survey-grade accuracy. Further, in order to have crosscutting deployment-ability, the system needed to be platform-independent, while to meet the needs of diverse end-users, a plug-n-go concept in the system design process was defined. Versatility and modularity were other important design criteria for the mapping system. Finally, the flexibility of the system to be adopted indoors and to have easily upgradable components was the last defining point in the design criteria set.

To meet these criteria and to ensure the successful development of the system, a smart integration scheme both on the HW and SW levels was implemented. Several processes were carried out, starting with the optimized selection of the multimodal sensors constituting the building blocks for a DG LiDAR-based mapping system. A balance was achieved between the cost, specifications and function-ability of the components to be used in the three mapping modes. A carefully designed housing for the system that allows for platform-switching capability was also developed, and the relative placements of the system components were set in an innovative way that facilitates the system deployment in stationary, mobile, and ULS modes. The efficient integration of sensors on the hardware level between the POS and LiDAR sensor by the precise synchronization utilizing the sequential PPS signal and the \$GPRMC message was implemented. The fusion of the multimodal sensors data streams was successfully carried out through the development of a new workflow. The new SW integration workflow allows the operator full control up to the sensor frame or sweep level. In addition, to decrease the turnaround time of the data products-generation, the system is parallel processing- and cloud computing-ready

A new mapping scheme was defined that successfully enables the utilization of an originally-built LiDAR scanner for dynamic environments to be used in stationary mode, thus allowing the crosscutting deployment-ability of the developed system in both kinematic and static-related mapping applications. The new mapping scheme overcomes the limited VFOV and vertical scan 
gaps associated with the LiDAR scanner by applying and measuring an incremental angular change to the system.

A rigorous non-linear uncertainty predictive model of the system uncertainty was derived. Moreover, aimed at to meet further end-user needs, a very low-cost variant of the developed system was introduced to be used in stationary and handheld modes. To test the functionality and operability of the developed system, the system realization was carried out covering different environments and being deployed in the full three mapping modes. Additionally, a comparative analysis of 3D point cloud generation through optical imagery SfM workflow and the LiDARbased ULS was implemented. The DSM generation using both approaches was carried out and assessed.

\subsubsection{System realization (Stationary mode)}

Applying the new mapping scheme allowed for system deployment in stationary mode. The system was used to map a building façade, with the system mounted on a fixed tripod. The LiDAR scanner limited VFOV and the vertical scan gaps between the scanning beams were substantially mitigated by applying the new mapping scheme. A very low-cost variant of the developed system was also introduced by using a (250 USD\$) MoCAP sensor to measure the system's incremental rotational angular change. The data from the LiDAR scanner and the GPS customer-grade receiver was synchronized and matched by the PPS and \$GPRMC message, and the synchronization and matching to the MoCAP sensor was achieved by using the NTP. The carefully designed housing and the new relative placement of the system components permitted the practical and easy deployment of the system in stationary mode. The qualitative assessment of the resultant point cloud proved the successful application of the system in stationary mode, as fine details were correctly mapped. The high-end Polaris system was used in a more quantitative assessment of the system. A RMSE of $0.06 \mathrm{~m}$ in the measured dimensions of common extracted features from the developed system point cloud and the Polaris point cloud was observed. The utilization of the APX-15 had a slightly better result

\subsubsection{System realization (MMS mode)}

System realization in MMS mode was implemented in a challenging urban environment, and a low-cost variant of the system with a MoCaP sensor was utilized in handheld mode. The developed system's light weight and small form-factor allowed for easy deployment in this mode. Also, the newly developed system components' data fusion workflow was applied to the mapped data. The ability to control the LiDAR sensor data up to the frame level, as introduced with the developed workflow, proved beneficial, as it allowed different levels of the mapped scene abstraction. Noise filtering and a recursive ICP applied to each data frame greatly augmented the originally collected data.

The developed system was deployed in MMS mode by mounting the system on a vehicle. The system's platform-switching capability (as a result of the carefully designed housing and the new relative placement of the system components) allowed the system to be easily switched to be mounted on a kinematic platform. Furthermore, the system's small form-factor and light weight enabled it to be mounted on a wide variety of vehicle types, from small sedans to SUVs. 
Additionally, street furniture and road surface markings were successfully mapped by the developed system. The reported uncertainty values for the position and attitude of the SBET were used in the developed uncertainty predictive model to estimate the georeferenced 3D point cloud geolocation uncertainty. The geolocation uncertainty was found to be $64 \mathrm{~cm}$. Deviations in the decimeter to meter range were observed between linear and planar primitives in the developed system's 3D point cloud and in a more accurate aerial LiDAR data available for the area mapped. The different perspective and horizontal/vertical datum between the aerial available data and the MMS data may explain the deviations.

The developed system's 3D point cloud was colorized through a new colorization approach that made use of already available imagery of the mapped area or imagery in the public domain for the mapped area. To register the 3D LiDAR point cloud to the 2D imagery, 3D-2D transformation was applied to transform the $3 \mathrm{D}$ point cloud to $2 \mathrm{D}$ imagery using the intensity values. This was followed by $2 \mathrm{D}-2 \mathrm{D}$ image registration using a semi-automated process. Once registered, the color information from the true color imagery was linked to the $3 \mathrm{D}$ point cloud and used in the colorization.

\subsubsection{System realization (ULS mode)}

The system was successfully deployed in ULS mode, with the system's small form-factor and light weight allowing for its deployment on a relatively small UAV platform. Moreover, the system's platform-switching capability (as a result of the carefully designed housing and the new relative placement of the system components) allowed the system to be easily switched to be used in ULS mode. EMI can have a drastic negative effect on the GNSS signal reception, as was experienced in one of our missions. The presence of many electronics within the limited size of the UAV adds to the challenge of EMI mitigation efforts.

Fine details were correctly mapped by the developed system in ULS mode. To measure the mapped 3D point spread of data, plane-fitting was applied to 3D point cloud samples, a mesh surface was created, and the normal distance between the points and the mesh surface was calculated. The standard deviation of the $3 \mathrm{D}$ point distances to the mesh surface was found to be $3-4 \mathrm{~cm}$ on different surface materials. The same procedure was applied using the high-end Polaris system data, with the standard deviation being $2-3 \mathrm{~cm}$ on different surface materials. The $3-4 \mathrm{~cm}$ range utilizing the developed system could be attributed to the precision of the system itself, while the variations in the Polaris case would likely be due to the nature of the irregular surfaces being mapped and the amount of detail the Polaris can map.

\subsubsection{SfM and ULS DSM generation}

The 3D point cloud of the developed system deployed in ULS was used to test the system's ability to generate derived data and the accuracy, such as DSM. Mean filtering and IDW interpolation were used to generate the DSM. A comparison of the system's performance and the low-cost SfM photogrammetry-based workflow was carried out. A UAV equipped with optical camera was used to capture overlapping images for the same mapped area. The SfM workflow provides an economical way to generate 3D point clouds and derived products such as DSM. The SfM was found to be problematic with tree-covered areas, as it was not generated in the resultant point cloud. This may be attributed to the challenging nature of finding key-matching points in 
overlapping images with the presence of trees. On the other hand, a wet pedestrian corridor within the mapped area was missed by the developed system. This may be attributed to the LiDAR scanner being operating in the $905 \mathrm{~nm}$, which is absorbable by water.

Relative height differences between the same feature pairs on the SfM and ULS datasets were compared independently to the Polaris-generated DSM, which was deemed the ground truth data. Some deviations in the decimeter range were observed between the Polaris dataset and the LiDARbased ULS developed system. The different data capturing perspectives may explain the deviation. On the other hand, the SfM showed slightly worse values and was unable to map tree-covered areas. The results showed that each approach application is challenging in a different type of environment, such that SfM is problematic in tree-covered areas, while LiDAR-based mapping degrades in wet areas.

The developed mapping system was successfully deployed in the three mapping modes, namely, stationary, mobile and ULS. The results attained, as assessed both qualitatively and quantitatively, proved the successful design and development of the DG LiDAR-based mapping system. The platform-switching ability and the plug-n-go concept was experienced through system realization in the different mapping modes. The new developed mapping scheme, the carefully designed housing, the new innovative relative placement of the system components, and the system's light weight and small form-factor all enabled and contributed to the crosscutting deployment-ability of the developed system.

\subsection{Key Contributions}

Some of the key contributions pertaining to the research presented are as follows:

- The generic system developed is a unique multipurpose 3D LiDAR-based mapping system designed and developed to allow the crosscutting deployment-ability among the three modes of operation.

- A new hardware-assembly approach was developed and implemented to allow such versatility and ease of deployment for the developed system.

- A new software integration approach for the system's multimodal data streams is developed allowing for full control of the scanned data up to the single frame level and is parallel processing- and cloud computing-ready

- A new application for an autonomous-vehicle-based sensor is tested in stationary mode and a completely new use, namely, the mapping of building façades, were developed 
- A new application of a UAV-based POS sensor in mobile and stationary modes was introduced and is successfully tested, paving the way for the use of autonomous-vehiclebased sensor as mobile mapping sensor in the three mapping modes

- A complete rigorous uncertainty predictive model for any LiDAR-based mapping system was developed and tested

- The novelty of the generic system architecture and the developed mapping system allowed patent filing; the research findings are patent pending.

- The purpose-built developed system has a substantial cost-reduction compared to the stateof-the-art commercial systems available. Furthermore, it has comparable performance metrics, while adding the unique crosscutting deployment-ability in the three mapping modes with maximum versatility and practicality.

\subsection{Recommendations for future work}

Suggestions and recommendations for future research work related to the present research work are listed below:

- A new data collection mechanism in stationary mode could be assessed by incrementally changing the height of the system to allow for the vertical gaps to be filled.

- The usage of two GNSS antennas could be assessed towards enhancing the accuracy of heading determination, especially in stationary and low velocity-related applications.

- Future work could also follow on the advantageous nature of LiDAR-based mapping and SfM workflow by testing different environments and performing a more thorough quantitative assessment. 


\section{Appendices}

\section{Appendix A: Derivation of the Developed System Uncertainty Predictive Model}

This appendix provides the complete derivation of the developed system uncertainty predictive model. The relation between the RSS, POS measurements and the georeferenced point cloud can be expressed through the direct georeferencing DG mathematical model. The LiDAR-based DG mathematical model can be expressed as a functional model through Equation A.1 and a stochastic model that is presented through Equation A.2. The complete derivation for the developed uncertainty predictive model is provided below.

$$
P_{l l}=r_{l l}(t)+R_{b}^{l l}(t) \cdot\left(R_{s}^{b} \cdot P_{s}(t)+l_{s}^{b}\right)
$$

Where

$P_{l l}$ is the $3 \mathrm{D}$ coordinates of the point in the local level frame

$r_{l l}(t)$ is the time dependant $3 \mathrm{D}$ coordinates of the body frame origin in the local level frame

$R_{b}^{l l}(t)$ is the time dependant rotation matrix from the body frame to the local level frame

(Defined through the time dependant roll, pitch, and yaw)

$R_{S}^{b}$ is the boresight rotation matrix between the body frame and the sensor frame

$P_{S}$ is the $3 \mathrm{D}$ point coordinates in the sensor frame

$l_{s}^{b}$ is the lever arm between the sensor CS frame origin and the body frame origin

While the stochastic part for the LiDAR-based DG mathematical model can be expressed through the law of error propagation. The uncertainty in the 3D coordinates of the georeferenced point cloud is related to the precision of the underlying VLP-16 and APX-15 measurements along with the precision in boresight angles and lever arms determination. Equation A.2 presents the stochastic part of the model.

$$
C_{s y s}=J C_{p a r} J^{T}
$$

Where

$C_{\text {sys }}$ is the variance-covariance matrix of the georeferenced 3D coordinates

It is a $[3 * 3]$ matrix 
$C_{p a r}$ is the variance-covariance matrix of the random variables in the DG Equation (the POS position, POS attitudes, range, scan angles, boresight angles, lever arms); it is a $\left[15^{*} 15\right]$ matrix

$J$ is the Jacobian matrix; the partial derivatives of the DG Equation with respect to the random variables involved; it is a $[3 * 15]$ matrix

In a more detailed representation and forward omitting the time dependent $(t)$ symbol just for clarity, the LiDAR-based DG Equation can be expressed as shown in Equation A.3.

$$
\left[\begin{array}{l}
X_{p} \\
Y_{p} \\
Z_{p}
\end{array}\right]_{l l}=\left[\begin{array}{c}
X_{b} \\
Y_{b} \\
Z_{b}
\end{array}\right]_{l l}+R_{\theta_{x}} R_{\theta_{y}} R_{\theta_{z}} *\left(R_{\tau_{x}} R_{\tau_{y}} R_{\tau_{z}} *\left[\begin{array}{c}
\rho \cos (\omega) \sin (\alpha) \\
\rho \cos (\omega) \cos (\alpha) \\
\rho \sin (\omega)
\end{array}\right]+\left[\begin{array}{c}
l x_{s}^{b} \\
l y_{s}^{b} \\
l z_{s}^{b}
\end{array}\right]\right)
$$

Where

$X_{p}, Y_{p}, Z_{p}$ are the $3 \mathrm{D}$ coordinates of the point in the local level frame

$X_{b}, Y_{b}, Z_{b}$ are the body frame origin time dependant $3 \mathrm{D}$ coordinates in the local level frame

$R_{\theta_{z}}$ is the time dependant rotation matrix from the body frame to the local level frame around the $\mathrm{z}$ axis with a yaw angle $\theta_{z}$

$R_{\theta_{y}}$ is the time dependant rotation matrix from the body frame to the local level frame around the y axis with a pitch angle $\theta_{y}$

$R_{\theta_{x}}$ is the time dependant rotation matrix from the body frame to the local level frame around the $\mathrm{x}$ axis with a roll angle $\theta_{x}$

$R_{\tau_{z}}$ is the rotation matrix from the sensor to the body frame around the $\mathrm{z}$ axis with a boresight angle $\tau_{z}$

$R_{\tau_{y}}$ is the rotation matrix from the sensor to the body frame around the y axis with a boresight angle $\tau_{y}$

$R_{\tau_{x}}$ is the rotation matrix from the sensor to the body frame around the $\mathrm{x}$ axis with a boresight angle $\tau_{x}$

$\omega$ is the laser beam vertical angle

$\rho$ is the range to the target

$\alpha$ is the laser horizontal angle

$l x_{s}^{b}, l y_{s}^{b}, l z_{s}^{b}$ are lever arm components between the sensor and the body frame origin 
The rotation sequence applied is a rotation around z-axis, followed by a rotation around the oncerotated y-axis, and lastly a rotation around the twice-rotated x-axis. The rotation matrices $R_{\theta_{z}}, R_{\theta_{y}}, R_{\theta_{x}} ; R_{\tau_{z}}, R_{\tau_{y}}, R_{\tau_{x}}$ can be more explicitly expressed as follows

$$
\begin{aligned}
R_{\theta_{z}} & =\left[\begin{array}{ccc}
\cos \theta_{z} & -\sin \theta_{z} & 0 \\
\sin \theta_{z} & \cos \theta_{z} & 0 \\
0 & 0 & 1
\end{array}\right] \\
R_{\theta_{y}} & =\left[\begin{array}{ccc}
\cos \theta_{y} & 0 & \sin \theta_{y} \\
0 & 1 & 0 \\
-\sin \theta_{y} & 0 & \cos \theta_{y}
\end{array}\right] \\
R_{\theta_{x}} & =\left[\begin{array}{ccc}
1 & 0 & 0 \\
0 & \cos \theta_{x} & -\sin \theta_{x} \\
0 & \sin \theta_{x} & \cos \theta_{x}
\end{array}\right] \\
R_{\tau_{z}} & =\left[\begin{array}{ccc}
\cos \tau_{z} & -\sin \tau_{z} & 0 \\
\sin \tau_{z} & \cos \tau_{z} & 0 \\
0 & 0 & 1
\end{array}\right] \\
R_{\tau_{y}} & =\left[\begin{array}{ccc}
\cos \tau_{y} & 0 & \sin \tau_{y} \\
0 & 1 & 0 \\
-\sin \tau_{y} & 0 & \cos \tau_{y}
\end{array}\right] \\
R_{\tau_{x}} & =\left[\begin{array}{ccc}
1 & 0 & 0 \\
0 & \cos \tau_{x} & -\sin \tau_{x} \\
0 & \sin \tau_{x} & \cos \tau_{x}
\end{array}\right]
\end{aligned}
$$

Thus

$$
\begin{gathered}
R_{b}^{l l}=R_{\theta_{x}} R_{\theta_{y}} R_{\theta_{z}}=\left[\begin{array}{ccc}
1 & 0 & 0 \\
0 & \cos \theta_{x} & -\sin \theta_{x} \\
0 & \sin \theta_{x} & \cos \theta_{x}
\end{array}\right]\left[\begin{array}{ccc}
\cos \theta_{y} & 0 & \sin \theta_{y} \\
0 & 1 & 0 \\
-\sin \theta_{y} & 0 & \cos \theta_{y}
\end{array}\right]\left[\begin{array}{ccc}
\cos \theta_{z} & -\sin \theta_{z} & 0 \\
\sin \theta_{z} & \cos \theta_{z} & 0 \\
0 & 0 & 1
\end{array}\right] \\
R_{b}^{l l}=\left[\begin{array}{ccc}
\cos \theta_{y} \cos \theta_{z} & -\cos \theta_{y} \sin \theta_{z} & \sin \theta_{y} \\
\cos \theta_{x} \sin \theta_{z}+\sin \theta_{x} \sin \theta_{y} \cos \theta_{z} & \cos \theta_{x} \cos \theta_{z}-\sin \theta_{x} \sin \theta_{y} \sin \theta_{z} & -\sin \theta_{x} \cos \theta_{y} \\
\sin \theta_{x} \sin \theta_{z}-\cos \theta_{x} \sin \theta_{y} \cos \theta_{z} & \sin \theta_{x} \cos \theta_{z}+\cos \theta_{x} \sin \theta_{y} \sin \theta_{z} & \cos \theta_{x} \cos \theta_{y}
\end{array}\right]
\end{gathered}
$$


And likewise $R_{s}^{b}$ can be defined as follows:

$$
\begin{gathered}
R_{s}^{b}=R_{\tau_{x}} R_{\tau_{y}} R_{\tau_{z}}=\left[\begin{array}{ccc}
1 & 0 & 0 \\
0 & \cos \tau_{x} & -\sin \tau_{x} \\
0 & \sin \tau_{x} & \cos \tau_{x}
\end{array}\right]\left[\begin{array}{ccc}
\cos \tau_{y} & 0 & \sin \tau_{y} \\
0 & 1 & 0 \\
-\sin \tau_{y} & 0 & \cos \tau_{y}
\end{array}\right]\left[\begin{array}{ccc}
\cos \tau_{z} & -\sin \tau_{z} & 0 \\
\sin \tau_{z} & \cos \tau_{z} & 0 \\
0 & 0 & 1
\end{array}\right] \\
R_{s}^{b}=\left[\begin{array}{ccc}
\cos \tau_{y} \cos \tau_{z} & \sin \tau_{y} \\
\cos \tau_{x} \sin \tau_{z}+\sin \tau_{x} \sin \tau_{y} \cos \tau_{z} & \cos \tau_{x} \cos \tau_{z}-\sin \tau_{x} \sin \tau_{y} \sin \tau_{z} & -\sin \tau_{x} \cos \tau_{y} \\
\sin \tau_{x} \sin \tau_{z}-\cos \tau_{x} \sin \tau_{y} \cos \tau_{z} & \sin \tau_{x} \cos \tau_{z}+\cos \tau_{x} \sin \tau_{y} \sin \tau_{z} & \cos \tau_{x} \cos \tau_{y}
\end{array}\right]
\end{gathered}
$$

Substituting $R_{b}^{l l}$ and $R_{s}^{b}$ in equation $A .3$ and denoting $\cos$ as c and $\sin$ as $s$ yields

$$
\begin{aligned}
& {\left[\begin{array}{l}
X_{p} \\
Y_{p} \\
Z_{p}
\end{array}\right]_{l l}=\left[\begin{array}{l}
X_{b} \\
Y_{b} \\
Z_{b}
\end{array}\right]_{l l}+\left[\begin{array}{ccc}
c \theta_{y} c \theta_{z} & -c \theta_{y} s \theta_{z} & s \theta_{y} \\
c \theta_{x} s \theta_{z}+s \theta_{x} s \theta_{y} c \theta_{z} & c \theta_{x} c \theta_{z}-s \theta_{x} s \theta_{y} s \theta_{z} & -s \theta_{x} c \theta_{y} \\
s \theta_{x} s \theta_{z}-c \theta_{x} s \theta_{y} c \theta_{z} & s \theta_{x} c \theta_{z}+c \theta_{x} s \theta_{y} s \theta_{z} & c \theta_{x} c \theta_{y}
\end{array}\right] *} \\
& \left(\left[\begin{array}{ccc}
c \tau_{y} c \tau_{z} s \tau_{z}+s \tau_{x} s \tau_{y} c \tau_{z} & c \tau_{x} c \tau_{z}-s \tau_{x} s \tau_{y} s \tau_{z} & -s \tau_{x} c \tau_{y} \\
s \tau_{x} s \tau_{z}-c \tau_{x} s \tau_{y} c \tau_{z} & s \tau_{x} c \tau_{z}+c \tau_{x} s \tau_{y} s \tau_{z} & c \tau_{x} c \tau_{y}
\end{array}\right] *\left[\begin{array}{c}
\rho c(\omega) s(\alpha) \\
\rho c(\omega) c(\alpha) \\
\rho s(\omega)
\end{array}\right]+\left[\begin{array}{c}
l x_{s}^{b} \\
l y_{s}^{b} \\
l z_{s}^{b}
\end{array}\right]\right)
\end{aligned}
$$


Calculating $R_{s}^{b} \cdot P_{S}(t)+l_{s}^{b}$ yields

$\left[\begin{array}{c}c \tau_{y} c \tau_{z} \rho c(\omega) s(\alpha)-c \tau_{y} s \tau_{z} \rho c(\omega) c(\alpha)+s \tau_{y} \rho s(\omega)+l x_{s}^{b} \\ c \tau_{x} s \tau_{z} \rho c(\omega) s(\alpha)+s \tau_{x} s \tau_{y} c \tau_{z} \rho c(\omega) s(\alpha)+c \tau_{x} c \tau_{z} \rho c(\omega) c(\alpha)-s \tau_{x} s \tau_{y} s \tau_{z} \rho c(\omega) c(\alpha)+-s \tau_{x} c \tau_{y} \rho s(\omega)+l y_{s}^{b} \\ s \tau_{x} s \tau_{z} \rho c(\omega) s(\alpha)-c \tau_{x} s \tau_{y} c \tau_{z} \rho c(\omega) s(\alpha)+s \tau_{x} c \tau_{z} \rho c(\omega) c(\alpha)+c \tau_{x} s \tau_{y} s \tau_{z} \rho c(\omega) c(\alpha)+c \tau_{x} c \tau_{y} \rho s(\omega)+l z_{s}^{b}\end{array}\right]$

And multiplying by $R_{b}^{l l}$

$\left[\begin{array}{ccc}c \theta_{y} c \theta_{z} & -c \theta_{y} s \theta_{z} & s \theta_{y} \\ c \theta_{x} s \theta_{z}+s \theta_{x} s \theta_{y} c \theta_{z} & c \theta_{x} c \theta_{z}-s \theta_{x} s \theta_{y} s \theta_{z} & -s \theta_{x} c \theta_{y} \\ s \theta_{x} s \theta_{z}-c \theta_{x} s \theta_{y} c \theta_{z} & s \theta_{x} c \theta_{z}+c \theta_{x} s \theta_{y} s \theta_{z} & c \theta_{x} c \theta_{y}\end{array}\right] *$

$\left[\begin{array}{c}c \tau_{y} c \tau_{z} \rho c(\omega) s(\alpha)-c \tau_{y} s \tau_{z} \rho c(\omega) c(\alpha)+s \tau_{y} \rho s(\omega)+l x_{s}^{b} \\ c \tau_{x} s \tau_{z} \rho c(\omega) s(\alpha)+s \tau_{x} s \tau_{y} c \tau_{z} \rho c(\omega) s(\alpha)+c \tau_{x} c \tau_{z} \rho c(\omega) c(\alpha)-s \tau_{x} s \tau_{y} s \tau_{z} \rho c(\omega) c(\alpha)+-s \tau_{x} c \tau_{y} \rho s(\omega)+l y_{s}^{b} \\ s \tau_{x} s \tau_{z} \rho c(\omega) s(\alpha)-c \tau_{x} s \tau_{y} c \tau_{z} \rho c(\omega) s(\alpha)+s \tau_{x} c \tau_{z} \rho c(\omega) c(\alpha)+c \tau_{x} s \tau_{y} s \tau_{z} \rho c(\omega) c(\alpha)+c \tau_{x} c \tau_{y} \rho s(\omega)+l z_{s}^{b}\end{array}\right]$

Yields $M=\left[\begin{array}{l}a_{11} \\ a_{12} \\ a_{13}\end{array}\right]$ where

$a_{11}=$

$c \theta_{y} c \theta_{z} c \tau_{y} c \tau_{z} \rho c(\omega) s(\alpha)-c \theta_{y} c \theta_{z} c \tau_{y} s \tau_{z} \rho c(\omega) c(\alpha)+c \theta_{y} c \theta_{z} s \tau_{y} \rho s(\omega)+c \theta_{y} c \theta_{z} l x_{s}^{b}-$

$c \theta_{y} s \theta_{z} c \tau_{x} s \tau_{z} \rho c(\omega) s(\alpha)-c \theta_{y} s \theta_{z} s \tau_{x} s \tau_{y} c \tau_{z} \rho c(\omega) s(\alpha)-c \theta_{y} s \theta_{z} c \tau_{x} c \tau_{z} \rho c(\omega) c(\alpha)+$

$c \theta_{y} s \theta_{z} s \tau_{x} s \tau_{y} s \tau_{z} \rho c(\omega) c(\alpha)+c \theta_{y} s \theta_{z} s \tau_{x} c \tau_{y} \rho s(\omega)-c \theta_{y} s \theta_{z} l y_{s}^{b}+\quad s \theta_{y} *$ $\left(s \tau_{x} s \tau_{z} \rho c(\omega) s(\alpha)-c \tau_{x} s \tau_{y} c \tau_{z} \rho c(\omega) s(\alpha)+s \tau_{x} c \tau_{z} \rho c(\omega) c(\alpha)+c \tau_{x} s \tau_{y} s \tau_{z} \rho c(\omega) c(\alpha)+\right.$ $\left.c \tau_{x} c \tau_{y} \rho s(\omega)+l z_{s}^{b}\right)$ 


$$
\begin{aligned}
& c \theta_{x} s \theta_{z} * \quad\left(c \tau_{y} c \tau_{z} \rho c(\omega) s(\alpha)-c \tau_{y} s \tau_{z} \rho c(\omega) c(\alpha)+s \tau_{y} \rho s(\omega)+l x_{s}^{b}\right)+s \theta_{x} s \theta_{y} c \theta_{z} * \\
& \left(c \tau_{y} c \tau_{z} \rho c(\omega) s(\alpha)-c \tau_{y} s \tau_{z} \rho c(\omega) c(\alpha)+s \tau_{y} \rho s(\omega)+l x_{s}^{b}\right)+c \theta_{x} c \theta_{z} * \\
& \left(c \tau_{x} s \tau_{z} \rho c(\omega) s(\alpha)+s \tau_{x} s \tau_{y} c \tau_{z} \rho c(\omega) s(\alpha)+c \tau_{x} c \tau_{z} \rho c(\omega) c(\alpha)-s \tau_{x} s \tau_{y} s \tau_{z} \rho c(\omega) c(\alpha)+\right. \\
& \left.-s \tau_{x} c \tau_{y} \rho s(\omega)+l y_{s}^{b}\right)-s \theta_{x} s \theta_{y} s \theta_{z} *\left(c \tau_{x} s \tau_{z} \rho c(\omega) s(\alpha)+s \tau_{x} s \tau_{y} c \tau_{z} \rho c(\omega) s(\alpha)+\right. \\
& \left.c \tau_{x} c \tau_{z} \rho c(\omega) c(\alpha)-s \tau_{x} s \tau_{y} s \tau_{z} \rho c(\omega) c(\alpha)+-s \tau_{x} c \tau_{y} \rho s(\omega)+l y_{s}^{b}\right)+-s \theta_{x} c \theta_{y} * \\
& \left(s \tau_{x} s \tau_{z} \rho c(\omega) s(\alpha)-c \tau_{x} s \tau_{y} c \tau_{z} \rho c(\omega) s(\alpha)+s \tau_{x} c \tau_{z} \rho c(\omega) c(\alpha)+c \tau_{x} s \tau_{y} s \tau_{z} \rho c(\omega) c(\alpha)+\right. \\
& \left.c \tau_{x} c \tau_{y} \rho s(\omega)+l z_{s}^{b}\right) \\
& a_{13}= \\
& s \theta_{x} s \theta_{z} *\left(c \tau_{y} c \tau_{z} \rho c(\omega) s(\alpha)-c \tau_{y} s \tau_{z} \rho c(\omega) c(\alpha)+s \tau_{y} \rho s(\omega)+l x_{s}^{b}\right)-c \theta_{x} s \theta_{y} c \theta_{z} * \\
& \left(c \tau_{y} c \tau_{z} \rho c(\omega) s(\alpha)-c \tau_{y} s \tau_{z} \rho c(\omega) c(\alpha)+s \tau_{y} \rho s(\omega)+l x_{s}^{b}\right)+s \theta_{x} c \theta_{z} * \\
& \left(c \tau_{x} s \tau_{z} \rho c(\omega) s(\alpha)+s \tau_{x} s \tau_{y} c \tau_{z} \rho c(\omega) s(\alpha)+c \tau_{x} c \tau_{z} \rho c(\omega) c(\alpha)-s \tau_{x} s \tau_{y} s \tau_{z} \rho c(\omega) c(\alpha)+\right. \\
& \left.-s \tau_{x} c \tau_{y} \rho s(\omega)+l y_{s}^{b}\right)+c \theta_{x} s \theta_{y} s \theta_{z} *\left(c \tau_{x} s \tau_{z} \rho c(\omega) s(\alpha)+s \tau_{x} s \tau_{y} c \tau_{z} \rho c(\omega) s(\alpha)+\right. \\
& \left.c \tau_{x} c \tau_{z} \rho c(\omega) c(\alpha)-s \tau_{x} s \tau_{y} s \tau_{z} \rho c(\omega) c(\alpha)+-s \tau_{x} c \tau_{y} \rho s(\omega)+l y_{s}^{b}\right)+c \theta_{x} c \theta_{y} * \\
& \left(s \tau_{x} s \tau_{z} \rho c(\omega) s(\alpha)-c \tau_{x} s \tau_{y} c \tau_{z} \rho c(\omega) s(\alpha)+s \tau_{x} c \tau_{z} \rho c(\omega) c(\alpha)+c \tau_{x} s \tau_{y} s \tau_{z} \rho c(\omega) c(\alpha)+\right. \\
& \left.c \tau_{x} c \tau_{y} \rho s(\omega)+l z_{s}^{b}\right)
\end{aligned}
$$

Thus, Equation A.3 can be rewritten in a simplified way as shown in Equation (A.4):

$$
\left[\begin{array}{l}
X_{p} \\
Y_{p} \\
Z_{p}
\end{array}\right]_{l l}=\left[\begin{array}{l}
X_{b} \\
Y_{b} \\
Z_{b}
\end{array}\right]_{l l}+\left[\begin{array}{l}
a_{11} \\
a_{12} \\
a_{13}
\end{array}\right]
$$

Rearranging

$$
\left[\begin{array}{l}
X_{p} \\
Y_{p} \\
Z_{p}
\end{array}\right]_{l l}=\left[\begin{array}{l}
X_{b}+a_{11} \\
Y_{b}+a_{12} \\
Z_{b}+a_{13}
\end{array}\right]
$$


Revisiting Equation A.2 to calculate the uncertainty attached with the developed mapping system.

$$
C_{\text {sys }}=J C_{\text {par }} J^{T}
$$

The 15 random variables involved in Equation A.2 can be grouped as shown in table A.1

Table A. 1: The LiDAR DG Equation 15 random variables

\begin{tabular}{|l|c|}
\hline Group & Variables \\
\hline POS position & $X_{b}, Y_{b}, Z_{b}$ \\
\hline POS attitudes(roll, pitch, yaw) & $\theta_{x}, \theta_{y}, \theta_{z}$ \\
\hline $\begin{array}{l}\text { LiDAR(range, vertical angle, horizontal scan } \\
\text { angle) }\end{array}$ & $\rho, \omega, \alpha$ \\
\hline $\begin{array}{l}\text { Calibration-based parameters: } \\
\text { (Boresight angles) } \\
\left.\text { (lever arm sensor and body frame } l_{s}^{b}\right)\end{array}$ & $\tau_{x}, \tau_{y}, \tau_{z}$ \\
& $l x_{s}^{b}, l y_{s}^{b}, l_{s}^{b}$ \\
\hline
\end{tabular}

The Jacobian matrix $J$ is computed through the partial derivatives of the DG Equation with respect to the 15 random variables involved. The $J$ matrix is a [3*15] matrix. The $J$ matrix would be given by Equation A.5. 
$J=\left[\begin{array}{lllllllllllllll}\frac{\partial X_{p}}{\partial X_{b}} & \frac{\partial X_{p}}{\partial Y_{b}} & \frac{\partial X_{p}}{\partial Z_{b}} & \frac{\partial X_{p}}{\partial \theta_{x}} & \frac{\partial X_{p}}{\partial \theta_{y}} & \frac{\partial X_{p}}{\partial \theta_{z}} & \frac{\partial X_{p}}{\partial \rho} & \frac{\partial X_{p}}{\partial \omega} & \frac{\partial X_{p}}{\partial \alpha} & \frac{\partial X_{p}}{\partial \tau_{x}} & \frac{\partial X_{p}}{\partial \tau_{y}} & \frac{\partial X_{p}}{\partial \tau_{z}} & \frac{\partial X_{p}}{\partial l_{s}^{b}} & \frac{\partial X_{p}}{\partial l y_{s}^{b}} & \frac{\partial X_{p}}{\partial l z_{s}^{b}} \\ \frac{\partial Y_{p}}{\partial X_{b}} & \frac{\partial Y_{p}}{\partial Y_{b}} & \frac{\partial Y_{p}}{\partial Z_{b}} \frac{\partial Y_{p}}{\partial \theta_{x}} & \frac{\partial Y_{p}}{\partial \theta_{y}} & \frac{\partial Y_{p}}{\partial \theta_{z}} & \frac{\partial Y_{p}}{\partial \rho} & \frac{\partial Y_{p}}{\partial \omega} & \frac{\partial Y_{p}}{\partial \alpha} & \frac{\partial Y_{p}}{\partial \tau_{x}} & \frac{\partial Y_{p}}{\partial \tau_{x}} & \frac{\partial Y_{p}}{\partial \tau_{z}} & \frac{\partial Y_{p}}{\partial l x_{s}^{b}} & \frac{\partial Y_{p}}{\partial l y_{s}^{b}} & \frac{\partial Y_{p}}{\partial z_{s}^{b}} \\ \frac{\partial Z_{p}}{\partial X_{b}} & \frac{\partial Z_{p}}{\partial Y_{b}} & \frac{\partial Z_{p}}{\partial Z_{b}} \frac{\partial Z_{p}}{\partial \theta_{x}} & \frac{\partial Z_{p}}{\partial \theta_{y}} & \frac{\partial Z_{p}}{\partial \theta_{z}} & \frac{\partial Z_{p}}{\partial \rho} & \frac{\partial Z_{p}}{\partial \omega} & \frac{\partial Z_{p}}{\partial \alpha} & \frac{\partial Z_{p}}{\partial \tau_{x}} & \frac{\partial Z_{p}}{\partial \tau_{x}} & \frac{\partial Z_{p}}{\partial \tau_{z}} & \frac{\partial Z_{p}}{\partial l_{s}^{b}} & \frac{\partial Z_{p}}{\partial l y_{s}^{b}} & \frac{\partial Z_{p}}{\partial l_{s}^{b}}\end{array}\right]$

Differentiating $X_{p} Y_{p} Z_{p}$ w.r.t the 15 random values, and evaluating at the initial values for the 15 random variables as listed in Table A.2 allows the population of the Jacobian matrix.

Table A. 2: The 15 random variables used in the error propagation and their initial values

\begin{tabular}{|l|c|l|}
\hline Group & Variables & Initial values \\
\hline POS position & $X_{b}, Y_{b}, Z_{b}$ & $0,0,0$ \\
\hline POS attitudes(roll, pitch, yaw) & $\theta_{x}, \theta_{y}, \theta_{z}$ & $0^{\circ}, 0^{\circ}, 0^{\circ}$ \\
\hline $\begin{array}{l}\text { LiDAR(range, vertical angle, horizontal } \\
\text { scan angle) }\end{array}$ & $\rho, \omega, \alpha$ & $100,0^{\circ}, 0$ \\
\hline $\begin{array}{l}\text { Calibration-based parameters: } \\
\text { (Boresight angles) }\end{array}$ & $\tau_{x}, \tau_{y}, \tau_{z}$ & $0,0^{\circ}, 0^{\circ}$ \\
$\quad$ (lever arm sensor and body frame $l_{s}^{b}$ ) & $l x_{s}^{b}, l y_{s}^{b}, l z_{s}$ & $0,0,0$ \\
\hline
\end{tabular}

The variance-covariance matrix $C_{\text {par }}$ can be populated with the 15 random variables variances which are typically reported as $(1 \sigma)$ and assuming that the variables are independent, the off diagonal elements for the $C_{\text {par }}$ matrix would be zero. Solving Equation A.2 yields the system uncertainty values for the georeferenced 3D point cloud positions. It is worth noting that the developed system uncertainty predictive model can be used to estimate the uncertainty of any DG LiDAR-based mapping system. 
Differentiating the $X_{p}$ Equation:

$$
\begin{gathered}
\frac{\partial X_{p}}{\partial X_{b}}=\frac{\partial\left(X_{b}+a_{11}\right)}{\partial X_{b}}=1 \\
\frac{\partial X_{p}}{\partial Y_{b}}=\frac{\partial\left(X_{b}+a_{11}\right)}{\partial Y_{b}}=0 \\
\frac{\partial X_{p}}{\partial Z_{b}}=\frac{\partial\left(X_{b}+a_{11}\right)}{\partial Z_{b}}=0 \\
\frac{\partial X_{p}}{\partial \theta_{x}}=\frac{\partial\left(X_{b}+a_{11}\right)}{\partial \theta_{x}}=\frac{\partial a_{11}}{\partial \theta_{x}}=0
\end{gathered}
$$




$$
\begin{aligned}
\frac{\partial X_{p}}{\partial \theta_{y}}=\frac{\partial\left(X_{b}+\right.}{\partial \theta_{y}}=\frac{\partial a_{11}}{\partial \theta_{y}} & \\
& =-s \theta_{y} \\
& *\left(c \theta_{z} c \tau_{y} c \tau_{z} \rho c(\omega) s(\alpha)-c \theta_{z} c \tau_{y} s \tau_{z} \rho c(\omega) c(\alpha)+c \theta_{z} s \tau_{y} \rho s(\omega)\right. \\
& +c \theta_{z} l x_{s}^{b}-s \theta_{z} c \tau_{x} s \tau_{z} \rho c(\omega) s(\alpha)-s \theta_{z} s \tau_{x} s \tau_{y} c \tau_{z} \rho c(\omega) s(\alpha) \\
& -s \theta_{z} c \tau_{x} c \tau_{z} \rho c(\omega) c(\alpha)+s \theta_{z} s \tau_{x} s \tau_{y} s \tau_{z} \rho c(\omega) c(\alpha)+s \theta_{z} s \tau_{x} c \tau_{y} \rho s(\omega) \\
& \left.-s \theta_{z} l y_{s}^{b}\right)+c \theta_{y} *\left(s \tau_{x} s \tau_{z} \rho c(\omega) s(\alpha)-c \tau_{x} s \tau_{y} c \tau_{z} \rho c(\omega) s(\alpha)\right. \\
& \left.+s \tau_{x} c \tau_{z} \rho c(\omega) c(\alpha)+c \tau_{x} s \tau_{y} s \tau_{z} \rho c(\omega) c(\alpha)+c \tau_{x} c \tau_{y} \rho s(\omega)+l z_{s}^{b}\right)
\end{aligned}
$$

$$
\begin{aligned}
\frac{\partial X_{p}}{\partial \theta_{z}}=\frac{\partial\left(X_{b}+\right.}{\partial \theta_{z}} & =\frac{\partial a_{11}}{\partial \theta_{z}} \\
& =-s \theta_{z} \\
& *\left(c \theta_{y} c \tau_{y} c \tau_{z} \rho c(\omega) s(\alpha)-c \theta_{y} c \tau_{y} s \tau_{z} \rho c(\omega) c(\alpha)+c \theta_{y} s \tau_{y} \rho s(\omega)+c \theta_{y} l x_{s}^{b}\right) \\
& +c \theta_{z} *\left(-c \theta_{y} c \tau_{x} s \tau_{z} \rho c(\omega) s(\alpha)-c \theta_{y} s \tau_{x} s \tau_{y} c \tau_{z} \rho c(\omega) s(\alpha)\right. \\
& -c \theta_{y} c \tau_{x} c \tau_{z} \rho c(\omega) c(\alpha)+c \theta_{y} s \tau_{x} s \tau_{y} s \tau_{z} \rho c(\omega) c(\alpha)+c \theta_{y} s \tau_{x} c \tau_{y} \rho s(\omega) \\
& \left.-c \theta_{y} l y_{s}^{b}\right)
\end{aligned}
$$

$$
\begin{aligned}
\frac{\partial X_{p}}{\partial \rho}=\frac{\partial\left(X_{b}+a_{11}\right)}{\partial \rho}=\frac{\partial a_{11}}{\partial \rho} & \\
& =c \theta_{y} c \theta_{z} c \tau_{y} c \tau_{z} c(\omega) s(\alpha)-c \theta_{y} c \theta_{z} c \tau_{y} s \tau_{z} c(\omega) c(\alpha)+c \theta_{y} c \theta_{z} s \tau_{y} s(\omega) \\
& -c \theta_{y} s \theta_{z} c \tau_{x} s \tau_{z} c(\omega) s(\alpha)-c \theta_{y} s \theta_{z} s \tau_{x} s \tau_{y} c \tau_{z} c(\omega) s(\alpha) \\
& -c \theta_{y} s \theta_{z} c \tau_{x} c \tau_{z} c(\omega) c(\alpha)+c \theta_{y} s \theta_{z} s \tau_{x} s \tau_{y} s \tau_{z} c(\omega) c(\alpha)+c \theta_{y} s \theta_{z} s \tau_{x} c \tau_{y} s(\omega) \\
& +s \theta_{y} s \tau_{x} s \tau_{z} c(\omega) s(\alpha)-s \theta_{y} c \tau_{x} s \tau_{y} c \tau_{z} c(\omega) s(\alpha)+s \theta_{y} s \tau_{x} c \tau_{z} c(\omega) c(\alpha) \\
& +s \theta_{y} c \tau_{x} s \tau_{y} s \tau_{z} c(\omega) c(\alpha)+s \theta_{y} c \tau_{x} c \tau_{y} s(\omega)
\end{aligned}
$$

$$
\begin{aligned}
\frac{\partial X_{p}}{\partial \omega}=\frac{\partial\left(X_{b}+\right.}{\partial \omega} & \left.a_{11}\right) \\
& =-s \omega \\
& *\left(c \theta_{y} c \theta_{z} c \tau_{y} c \tau_{z} \rho s(\alpha)-c \theta_{y} c \theta_{z} c \tau_{y} s \tau_{z} \rho c(\alpha)-c \theta_{y} s \theta_{z} c \tau_{x} s \tau_{z} \rho s(\alpha)\right. \\
& -c \theta_{y} s \theta_{z} s \tau_{x} s \tau_{y} c \tau_{z} \rho s(\alpha)-c \theta_{y} s \theta_{z} c \tau_{x} c \tau_{z} \rho c(\alpha)+c \theta_{y} s \theta_{z} s \tau_{x} s \tau_{y} s \tau_{z} \rho c(\alpha) \\
& +s \theta_{y} s \tau_{x} s \tau_{z} \rho s(\alpha)-s \theta_{y} c \tau_{x} s \tau_{y} c \tau_{z} \rho s(\alpha)+s \theta_{y} s \tau_{x} c \tau_{z} \rho c(\alpha) \\
& \left.+s \theta_{y} c \tau_{x} s \tau_{y} s \tau_{z} \rho c(\alpha)\right)+c \omega *\left(c \theta_{y} c \theta_{z} s \tau_{y} \rho+c \theta_{y} s \theta_{z} s \tau_{x} c \tau_{y} \rho+s \theta_{y} c \tau_{x} c \tau_{y} \rho\right)
\end{aligned}
$$




$$
\begin{aligned}
\frac{\partial X_{p}}{\partial \alpha}=\frac{\partial\left(X_{b}+\right.}{\partial \alpha} & \left.a_{11}\right) \\
& =c \alpha \\
& *\left(c \theta_{y} c \theta_{z} c \tau_{y} c \tau_{z} \rho c(\omega)-c \theta_{y} s \theta_{z} c \tau_{x} s \tau_{z} \rho c(\omega)-c \theta_{y} s \theta_{z} s \tau_{x} s \tau_{y} c \tau_{z} \rho c(\omega)\right. \\
& \left.+s \theta_{y} s \tau_{x} s \tau_{z} \rho c(\omega)-s \theta_{y} c \tau_{x} s \tau_{y} c \tau_{z} \rho c(\omega)\right)-s \alpha *\left(-c \theta_{y} c \theta_{z} c \tau_{y} s \tau_{z} \rho c(\omega)\right. \\
& -c \theta_{y} s \theta_{z} c \tau_{x} c \tau_{z} \rho c(\omega)+c \theta_{y} s \theta_{z} s \tau_{x} s \tau_{y} s \tau_{z} \rho c(\omega)+s \theta_{y} s \tau_{x} c \tau_{z} \rho c(\omega) \\
& \left.+s \theta_{y} c \tau_{x} s \tau_{y} s \tau_{z} \rho c(\omega)\right)
\end{aligned}
$$

$$
\begin{aligned}
\frac{\partial X_{p}}{\partial \tau_{x}}=\frac{\partial\left(X_{b}+\right.}{\partial \tau_{x}}=\frac{\partial a_{11}}{\partial \tau_{x}} & =-s \tau_{x} \\
& *\left(-c \theta_{y} s \theta_{z} s \tau_{z} \rho c(\omega) s(\alpha)-c \theta_{y} s \theta_{z} c \tau_{z} \rho c(\omega) c(\alpha)-s \theta_{y} s \tau_{y} c \tau_{z} \rho c(\omega) s(\alpha)\right. \\
& \left.+s \theta_{y} s \tau_{y} s \tau_{z} \rho c(\omega) c(\alpha)+s \theta_{y} c \tau_{y} \rho s(\omega)\right)+c \tau_{x} *\left(c \theta_{y} s \theta_{z} s \tau_{y} c \tau_{z} \rho c(\omega) s(\alpha)\right. \\
& +c \theta_{y} s \theta_{z} s \tau_{y} s \tau_{z} \rho c(\omega) c(\alpha)+c \theta_{y} s \theta_{z} c \tau_{y} \rho s(\omega)+s \theta_{y} s \tau_{z} \rho c(\omega) s(\alpha) \\
& \left.+s \theta_{y} c \tau_{z} \rho c(\omega) c(\alpha)\right)
\end{aligned}
$$

$$
\begin{aligned}
\frac{\partial X_{p}}{\partial \tau_{y}}=\frac{\partial\left(X_{b}+\right.}{\partial \tau_{y}} & =\frac{\partial a_{11}}{\partial \tau_{y}} \\
& =-s \tau_{y} \\
& *\left(c \theta_{y} c \theta_{z} c \tau_{z} \rho c(\omega) s(\alpha)-c \theta_{y} c \theta_{z} s \tau_{z} \rho c(\omega) c(\alpha)+c \theta_{y} s \theta_{z} s \tau_{x} \rho s(\omega)\right. \\
& \left.+s \theta_{y} c \tau_{x} \rho s(\omega)\right)+c \tau_{y} *\left(c \theta_{y} c \theta_{z} \rho s(\omega)-c \theta_{y} s \theta_{z} s \tau_{x} c \tau_{z} \rho c(\omega) s(\alpha)\right. \\
& \left.+c \theta_{y} s \theta_{z} s \tau_{x} s \tau_{z} \rho c(\omega) c(\alpha)-s \theta_{y} c \tau_{x} c \tau_{z} \rho c(\omega) s(\alpha)+s \theta_{y} c \tau_{x} s \tau_{z} \rho c(\omega) c(\alpha)\right)
\end{aligned}
$$

$$
\begin{aligned}
& \frac{\partial X_{p}}{\partial \tau_{z}}=\frac{\partial\left(X_{b}+a_{11}\right)}{\partial \tau_{z}}=\frac{\partial a_{11}}{\partial \tau_{z}} \\
& =-s \tau_{z} \\
& *\left(c \theta_{y} c \theta_{z} c \tau_{y} \rho c(\omega) s(\alpha)-c \theta_{y} s \theta_{z} s \tau_{x} s \tau_{y} \rho c(\omega) s(\alpha)-c \theta_{y} s \theta_{z} c \tau_{x} \rho c(\omega) c(\alpha)\right. \\
& \left.-s \theta_{y} c \tau_{x} s \tau_{y} \rho c(\omega) s(\alpha)+s \theta_{y} s \tau_{x} \rho c(\omega) c(\alpha)\right)+c \tau_{z} *\left(-c \theta_{y} c \theta_{z} c \tau_{y} \rho c(\omega) c(\alpha)\right. \\
& -c \theta_{y} s \theta_{z} c \tau_{x} \rho c(\omega) s(\alpha)+c \theta_{y} s \theta_{z} s \tau_{x} s \tau_{y} \rho c(\omega) c(\alpha)+s \theta_{y} s \tau_{x} \rho c(\omega) s(\alpha) \\
& \left.+s \theta_{y} c \tau_{x} s \tau_{y} \rho c(\omega) c(\alpha)\right) \\
& \frac{\partial X_{p}}{\partial l x_{s}^{b}}=\frac{\partial\left(X_{b}+a_{11}\right)}{\partial l x_{s}^{b}}=\frac{\partial a_{11}}{\partial l x_{s}^{b}}=c \theta_{y} c \theta_{z}
\end{aligned}
$$




$$
\begin{gathered}
\frac{\partial X_{p}}{\partial l y_{s}^{b}}=\frac{\partial\left(X_{b}+a_{11}\right)}{\partial l y_{s}^{b}}=\frac{\partial a_{11}}{\partial l y_{s}^{b}}=-c \theta_{y} s \theta_{z} \\
\frac{\partial X_{p}}{\partial l z_{s}^{b}}=\frac{\partial\left(X_{b}+a_{11}\right)}{\partial l z_{s}^{b}}=\frac{\partial a_{11}}{\partial l z_{s}^{b}}=s \theta_{y}
\end{gathered}
$$

Differentiating the $Y_{p}$ Equation:

$$
\begin{aligned}
& \frac{\partial Y_{p}}{\partial X_{b}}=\frac{\partial\left(Y_{b}+a_{12}\right)}{\partial X_{b}}=0 \\
& \frac{\partial Y_{p}}{\partial Y_{b}}=\frac{\partial\left(Y_{b}+a_{12}\right)}{\partial Y_{b}}=1 \\
& \frac{\partial Y_{p}}{\partial Z_{b}}=\frac{\partial\left(Y_{b}+a_{12}\right)}{\partial Z_{b}}=0
\end{aligned}
$$




$$
\begin{aligned}
& \frac{\partial Y_{p}}{\partial \theta_{x}}=\frac{\partial\left(Y_{b}+a_{12}\right)}{\partial \theta_{x}}=\frac{\partial a_{12}}{\partial \theta_{x}} \\
& =-s \theta_{x} s \theta_{z} *\left(c \tau_{y} c \tau_{z} \rho c(\omega) s(\alpha)-c \tau_{y} s \tau_{z} \rho c(\omega) c(\alpha)+s \tau_{y} \rho s(\omega)+l x_{s}^{b}\right) \\
& -s \theta_{x} c \theta_{z} \\
& *\left(c \tau_{x} s \tau_{z} \rho c(\omega) s(\alpha)+s \tau_{x} s \tau_{y} c \tau_{z} \rho c(\omega) s(\alpha)+c \tau_{x} c \tau_{z} \rho c(\omega) c(\alpha)\right. \\
& \left.-s \tau_{x} s \tau_{y} s \tau_{z} \rho c(\omega) c(\alpha)+-s \tau_{x} c \tau_{y} \rho s(\omega)+l y_{s}^{b}\right)-s \theta_{x} s \theta_{y} s \theta_{z} \\
& *\left(c \tau_{x} s \tau_{z} \rho c(\omega) s(\alpha)+s \tau_{x} s \tau_{y} c \tau_{z} \rho c(\omega) s(\alpha)+c \tau_{x} c \tau_{z} \rho c(\omega) c(\alpha)\right. \\
& \left.-s \tau_{x} s \tau_{y} s \tau_{z} \rho c(\omega) c(\alpha)+-s \tau_{x} c \tau_{y} \rho s(\omega)+l y_{s}^{b}\right)+c \theta_{x} s \theta_{y} c \theta_{z} \\
& *\left(c \tau_{y} c \tau_{z} \rho c(\omega) s(\alpha)-c \tau_{y} s \tau_{z} \rho c(\omega) c(\alpha)+s \tau_{y} \rho s(\omega)+l x_{s}^{b}\right)-c \theta_{x} s \theta_{y} s \theta_{z} \\
& *\left(c \tau_{x} s \tau_{z} \rho c(\omega) s(\alpha)+s \tau_{x} s \tau_{y} c \tau_{z} \rho c(\omega) s(\alpha)+c \tau_{x} c \tau_{z} \rho c(\omega) c(\alpha)\right. \\
& \left.-s \tau_{x} s \tau_{y} s \tau_{z} \rho c(\omega) c(\alpha)+-s \tau_{x} c \tau_{y} \rho s(\omega)+l y_{s}^{b}\right)-c \theta_{x} c \theta_{y} *\left(s \tau_{x} s \tau_{z} \rho c(\omega) s(\alpha)\right. \\
& -c \tau_{x} s \tau_{y} c \tau_{z} \rho c(\omega) s(\alpha)+s \tau_{x} c \tau_{z} \rho c(\omega) c(\alpha)+c \tau_{x} s \tau_{y} s \tau_{z} \rho c(\omega) c(\alpha) \\
& \left.+c \tau_{x} c \tau_{y} \rho s(\omega)+l z_{s}^{b}\right) \\
& \frac{\partial Y_{p}}{\partial \theta_{y}}=\frac{\partial\left(Y_{b}+a_{12}\right)}{\partial \theta_{y}}=\frac{\partial a_{12}}{\partial \theta_{y}} \\
& =c \theta_{y} s \theta_{x} c \theta_{z} *\left(c \tau_{y} c \tau_{z} \rho c(\omega) s(\alpha)-c \tau_{y} s \tau_{z} \rho c(\omega) c(\alpha)+s \tau_{y} \rho s(\omega)+l x_{s}^{b}\right) \\
& -c \theta_{y} s \theta_{x} s \theta_{z} \\
& *\left(c \tau_{x} s \tau_{z} \rho c(\omega) s(\alpha)+s \tau_{x} s \tau_{y} c \tau_{z} \rho c(\omega) s(\alpha)+c \tau_{x} c \tau_{z} \rho c(\omega) c(\alpha)\right. \\
& \left.-s \tau_{x} s \tau_{y} s \tau_{z} \rho c(\omega) c(\alpha)+-s \tau_{x} c \tau_{y} \rho s(\omega)+l y_{s}^{b}\right)+s \theta_{y} s \theta_{x} *\left(s \tau_{x} s \tau_{z} \rho c(\omega) s(\alpha)\right. \\
& -c \tau_{x} s \tau_{y} c \tau_{z} \rho c(\omega) s(\alpha)+s \tau_{x} c \tau_{z} \rho c(\omega) c(\alpha)+c \tau_{x} s \tau_{y} s \tau_{z} \rho c(\omega) c(\alpha) \\
& \left.+c \tau_{x} c \tau_{y} \rho s(\omega)+l z_{s}^{b}\right) \\
& \frac{\partial Y_{p}}{\partial \theta_{z}}=\frac{\partial\left(Y_{b}+a_{12}\right)}{\partial \theta_{z}}=\frac{\partial a_{12}}{\partial \theta_{z}}=c \theta_{z} c \theta_{x} *\left(c \tau_{y} c \tau_{z} \rho c(\omega) s(\alpha)-c \tau_{y} s \tau_{z} \rho c(\omega) c(\alpha)+s \tau_{y} \rho s(\omega)+\right. \\
& \left.l x_{s}^{b}\right)-s \theta_{z} s \theta_{x} s \theta_{y} *\left(c \tau_{y} c \tau_{z} \rho c(\omega) s(\alpha)-c \tau_{y} s \tau_{z} \rho c(\omega) c(\alpha)+s \tau_{y} \rho s(\omega)+l x_{s}^{b}\right)-s \theta_{z} c \theta_{x} * \\
& \left(c \tau_{x} s \tau_{z} \rho c(\omega) s(\alpha)+s \tau_{x} s \tau_{y} c \tau_{z} \rho c(\omega) s(\alpha)+c \tau_{x} c \tau_{z} \rho c(\omega) c(\alpha)-s \tau_{x} s \tau_{y} s \tau_{z} \rho c(\omega) c(\alpha)+\right. \\
& \left.-s \tau_{x} c \tau_{y} \rho s(\omega)+l y_{s}^{b}\right)-c \theta_{z} s \theta_{x} s \theta_{y} *\left(c \tau_{x} s \tau_{z} \rho c(\omega) s(\alpha)+s \tau_{x} s \tau_{y} c \tau_{z} \rho c(\omega) s(\alpha)+\right. \\
& \left.c \tau_{x} c \tau_{z} \rho c(\omega) c(\alpha)-s \tau_{x} s \tau_{y} s \tau_{z} \rho c(\omega) c(\alpha)+-s \tau_{x} c \tau_{y} \rho s(\omega)+l y_{s}^{b}\right)
\end{aligned}
$$




$$
\begin{aligned}
& \frac{\partial Y_{p}}{\partial \rho}=\frac{\partial\left(Y_{b}+a_{12}\right)}{\partial \rho} \\
& =c \theta_{x} s \theta_{z} *\left(c \tau_{y} c \tau_{z} c(\omega) s(\alpha)-c \tau_{y} s \tau_{z} c(\omega) c(\alpha)+s \tau_{y} s(\omega)\right)+s \theta_{x} s \theta_{y} c \theta_{z} \\
& *\left(c \tau_{y} c \tau_{z} c(\omega) s(\alpha)-c \tau_{y} s \tau_{z} c(\omega) c(\alpha)+s \tau_{y} s(\omega)\right)+c \theta_{x} c \theta_{z} *\left(c \tau_{x} s \tau_{z} c(\omega) s(\alpha)\right. \\
& +s \tau_{x} s \tau_{y} c \tau_{z} c(\omega) s(\alpha)+c \tau_{x} c \tau_{z} c(\omega) c(\alpha)-s \tau_{x} s \tau_{y} s \tau_{z} c(\omega) c(\alpha) \\
& \left.+-s \tau_{x} c \tau_{y} s(\omega)\right)-s \theta_{x} s \theta_{y} s \theta_{z} *\left(c \tau_{x} s \tau_{z} c(\omega) s(\alpha)+s \tau_{x} s \tau_{y} c \tau_{z} c(\omega) s(\alpha)\right. \\
& \left.+c \tau_{x} c \tau_{z} c(\omega) c(\alpha)-s \tau_{x} s \tau_{y} s \tau_{z} c(\omega) c(\alpha)+-s \tau_{x} c \tau_{y} s(\omega)\right)+-s \theta_{x} c \theta_{y} \\
& *\left(s \tau_{x} s \tau_{z} c(\omega) s(\alpha)-c \tau_{x} s \tau_{y} c \tau_{z} c(\omega) s(\alpha)+s \tau_{x} c \tau_{z} c(\omega) c(\alpha)\right. \\
& \left.+c \tau_{x} s \tau_{y} s \tau_{z} c(\omega) c(\alpha)+c \tau_{x} c \tau_{y} s(\omega)+l z_{s}^{b}\right) \\
& \frac{\partial Y_{p}}{\partial \omega}=\frac{\partial\left(Y_{b}+a_{12}\right)}{\partial \omega} \\
& =-s \omega c \theta_{x} s \theta_{z} *\left(c \tau_{y} c \tau_{z} \rho s(\alpha)-c \tau_{y} s \tau_{z} \rho c(\alpha)\right)-s \omega s \theta_{x} s \theta_{y} c \theta_{z} \\
& *\left(c \tau_{y} c \tau_{z} \rho s(\alpha)-c \tau_{y} s \tau_{z} \rho c(\alpha)\right)-s \omega c \theta_{x} c \theta_{z} \\
& *\left(c \tau_{x} s \tau_{z} \rho s(\alpha)+s \tau_{x} s \tau_{y} c \tau_{z} \rho s(\alpha)+c \tau_{x} c \tau_{z} \rho c(\alpha)-s \tau_{x} s \tau_{y} s \tau_{z} \rho c(\alpha)\right) \\
& +s \omega s \theta_{x} s \theta_{y} s \theta_{z} \\
& *\left(c \tau_{x} s \tau_{z} \rho s(\alpha)+s \tau_{x} s \tau_{y} c \tau_{z} \rho s(\alpha)+c \tau_{x} c \tau_{z} \rho c(\alpha)-s \tau_{x} s \tau_{y} s \tau_{z} \rho c(\alpha)\right) \\
& +s \omega s \theta_{x} c \theta_{y} \\
& *\left(s \tau_{x} s \tau_{z} \rho s(\alpha)-c \tau_{x} s \tau_{y} c \tau_{z} \rho s(\alpha)+s \tau_{x} c \tau_{z} \rho c(\alpha)+c \tau_{x} s \tau_{y} s \tau_{z} \rho c(\alpha)\right) \\
& +c \omega c \theta_{x} s \theta_{z} s \tau_{y} \rho+c \omega s \theta_{x} s \theta_{y} c \theta_{z} s \tau_{y} \rho-c \omega c \theta_{x} c \theta_{z} s \tau_{x} c \tau_{y} \rho \\
& +c \omega s \theta_{x} s \theta_{y} s \theta_{z} s \tau_{x} c \tau_{y} \rho-c \omega s \theta_{x} c \theta_{y} c \tau_{x} c \tau_{y} \rho \\
& \frac{\partial Y_{p}}{\partial \alpha}=\frac{\partial\left(Y_{b}+a_{12}\right)}{\partial \alpha} \\
& =c \alpha c \theta_{x} s \theta_{z} c \tau_{y} c \tau_{z} \rho c(\omega)+s \alpha c \theta_{x} s \theta_{z} c \tau_{y} s \tau_{z} \rho c(\omega)+c \alpha s \theta_{x} s \theta_{y} c \theta_{z} c \tau_{y} c \tau_{z} \rho c(\omega) \\
& +s \alpha s \theta_{x} s \theta_{y} c \theta_{z} c \tau_{y} s \tau_{z} \rho c(\omega)+c \alpha c \theta_{x} c \theta_{z} *\left(c \tau_{x} s \tau_{z} \rho c(\omega)+s \tau_{x} s \tau_{y} c \tau_{z} \rho c(\omega)\right. \\
& -s \alpha c \theta_{x} c \theta_{z} *\left(c \tau_{x} c \tau_{z} \rho c(\omega)-s \tau_{x} s \tau_{y} s \tau_{z} \rho c(\omega)\right)-c \alpha s \theta_{x} s \theta_{y} s \theta_{z} \\
& *\left(c \tau_{x} s \tau_{z} \rho c(\omega)+s \tau_{x} s \tau_{y} c \tau_{z} \rho c(\omega)\right)+s \alpha s \theta_{x} s \theta_{y} s \theta_{z} \\
& *\left(c \tau_{x} c \tau_{z} \rho c(\omega)-s \tau_{x} s \tau_{y} s \tau_{z} \rho c(\omega)\right)-c \alpha s \theta_{x} c \theta_{y} *\left(s \tau_{x} s \tau_{z} \rho c(\omega)\right. \\
& -c \tau_{x} s \tau_{y} c \tau_{z} \rho c(\omega)+s \alpha s \theta_{x} c \theta_{y} *\left(s \tau_{x} c \tau_{z} \rho c(\omega)+c \tau_{x} s \tau_{y} s \tau_{z} \rho c(\omega)\right)
\end{aligned}
$$




$$
\begin{aligned}
\frac{\partial Y_{p}}{\partial \tau_{x}}=\frac{\partial\left(Y_{b}+\right.}{\partial \tau_{x}} & \\
& =-s \tau_{x} c \theta_{x} c \theta_{z} *\left(s \tau_{z} \rho c(\omega) s(\alpha)+c \tau_{z} \rho c(\omega) c(\alpha)\right)+c \tau_{x} c \theta_{x} c \theta_{z} \\
& *\left(s \tau_{y} c \tau_{z} \rho c(\omega) s(\alpha)-s \tau_{y} s \tau_{z} \rho c(\omega) c(\alpha)-c \tau_{y} \rho s(\omega)\right)+s \tau_{x} s \theta_{x} s \theta_{y} s \theta_{z} \\
& *\left(s \tau_{z} \rho c(\omega) s(\alpha)+c \tau_{z} \rho c(\omega) c(\alpha)\right)-c \tau_{x} s \theta_{x} s \theta_{y} s \theta_{z} \\
& *\left(s \tau_{y} c \tau_{z} \rho c(\omega) s(\alpha)-s \tau_{y} s \tau_{z} \rho c(\omega) c(\alpha)-c \tau_{y} \rho s(\omega)\right)-c \tau_{x} s \theta_{x} c \theta_{y} \\
& *\left(s \tau_{z} \rho c(\omega) s(\alpha)+c \tau_{z} \rho c(\omega) c(\alpha)\right)+s \theta_{x} c \theta_{y}\left(-c \tau_{x} s \tau_{y} c \tau_{z} \rho c(\omega) s(\alpha)\right. \\
& \left.+s \tau_{y} s \tau_{z} \rho c(\omega) c(\alpha)+c \tau_{y} \rho s(\omega)\right)
\end{aligned}
$$

$$
\begin{aligned}
\frac{\partial Y_{p}}{\partial \tau_{y}}=\frac{\partial\left(Y_{b}+\right.}{\partial \tau_{y}} & \\
& =-s \tau_{y} c \theta_{x} s \theta_{z}\left(c \tau_{z} \rho c(\omega) s(\alpha)-s \tau_{z} \rho c(\omega) c(\alpha)\right) \\
& -s \tau_{y} s \theta_{x} s \theta_{y} c \theta_{z}\left(c \tau_{z} \rho c(\omega) s(\alpha)-s \tau_{z} \rho c(\omega) c(\alpha)\right)-s \tau_{y} c \theta_{x} c \theta_{z} \\
& *\left(c \tau_{x} s \tau_{z} \rho c(\omega) s(\alpha)+c \tau_{x} c \tau_{z} \rho c(\omega) c(\alpha)-s \tau_{x} c \tau_{y} \rho s(\omega)\right)-s \tau_{y} s \theta_{x} s \theta_{y} s \theta_{z} \\
& *\left(-s \tau_{x} \rho s(\omega)\right)+s \tau_{y} s \theta_{x} c \theta_{y} c \tau_{x} \rho s(\omega)+c \tau_{y} c \theta_{x} s \theta_{z} s \tau_{y} \rho s(\omega) \\
& +c \tau_{y} s \theta_{x} s \theta_{y} c \theta_{z} s \tau_{y} \rho s(\omega)+c \tau_{y} c \theta_{x} c \theta_{z} \\
& *\left(s \tau_{x} c \tau_{z} \rho c(\omega) s(\alpha)-s \tau_{x} s \tau_{z} \rho c(\omega) c(\alpha)\right)-c \tau_{y} s \theta_{x} s \theta_{y} s \theta_{z} \\
& *\left(s \tau_{x} c \tau_{z} \rho c(\omega) s(\alpha)-s \tau_{x} s \tau_{z} \rho c(\omega) c(\alpha)\right)-c \tau_{y} s \theta_{x} c \theta_{y} *\left(-c \tau_{x} c \tau_{z} \rho c(\omega) s(\alpha)\right. \\
& \left.+c \tau_{x} s \tau_{z} \rho c(\omega) c(\alpha)\right)
\end{aligned}
$$

$$
\begin{aligned}
\frac{\partial Y_{p}}{\partial \tau_{z}}=\frac{\partial\left(Y_{b}+\right.}{\partial \tau_{z}} & \left.a_{12}\right) \\
& =-s \tau_{z} c \theta_{x} s \theta_{z} c \tau_{y} \rho c(\omega) s(\alpha)-s \tau_{z} s \theta_{x} s \theta_{y} c \theta_{z} c \tau_{y} \rho c(\omega) s(\alpha)-s \tau_{z} c \theta_{x} c \theta_{z} \\
& *\left(s \tau_{x} s \tau_{y} \rho c(\omega) s(\alpha)+c \tau_{x} \rho c(\omega) c(\alpha)\right)+s \tau_{z} s \theta_{x} s \theta_{y} s \theta_{z} \\
& *\left(s \tau_{x} s \tau_{y} \rho c(\omega) s(\alpha)+c \tau_{x} \rho c(\omega) c(\alpha)\right) \\
& +s \tau_{z} s \theta_{x} c \theta_{y}\left(-c \tau_{x} s \tau_{y} \rho c(\omega) s(\alpha)+s \tau_{x} \rho c(\omega) c(\alpha)\right) \\
& -c \tau_{z} c \theta_{x} s \theta_{z} c \tau_{y} s \tau_{z} \rho c(\omega) c(\alpha)-c \tau_{z} s \theta_{x} s \theta_{y} c \theta_{z} c \tau_{y} s \tau_{z} \rho c(\omega) c(\alpha)+c \tau_{z} c \theta_{x} c \theta_{z} \\
& *\left(c \tau_{x} \rho c(\omega) s(\alpha)-s \tau_{x} s \tau_{y} \rho c(\omega) c(\alpha)\right)-c \tau_{z} s \theta_{x} s \theta_{y} s \theta_{z} \\
& *\left(c \tau_{x} \rho c(\omega) s(\alpha)-s \tau_{x} s \tau_{y} \rho c(\omega) c(\alpha)\right)-c \tau_{z} s \theta_{x} c \theta_{y} *\left(s \tau_{x} \rho c(\omega) s(\alpha)\right. \\
& \left.+c \tau_{x} s \tau_{y} \rho c(\omega) c(\alpha)\right) \\
& \frac{\partial Y_{p}}{\partial l x_{s}^{b}}=\frac{\partial\left(Y_{b}+a_{12}\right)}{\partial l x_{s}^{b}}=c \theta_{x} s \theta_{z}+s \theta_{x} s \theta_{y} c \theta_{z}
\end{aligned}
$$




$$
\begin{gathered}
\frac{\partial Y_{p}}{\partial l y_{s}^{b}}=\frac{\partial\left(Y_{b}+a_{12}\right)}{\partial l y_{s}^{b}}=c \theta_{x} c \theta_{z}-s \theta_{x} s \theta_{y} s \theta_{z} \\
\frac{\partial Y_{p}}{\partial l z_{s}^{b}}=\frac{\partial\left(Y_{b}+a_{12}\right)}{\partial l z_{s}^{b}}=-s \theta_{x} c \theta_{y}
\end{gathered}
$$

Differentiating the $Z_{p}$ Equation:

$$
\begin{aligned}
& \frac{\partial Z_{p}}{\partial X_{b}}=\frac{\partial\left(Z_{b}+a_{13}\right)}{\partial X_{b}}=0 \\
& \frac{\partial Z_{p}}{\partial Y_{b}}=\frac{\partial\left(Z_{b}+a_{13}\right)}{\partial Y_{b}}=0 \\
& \frac{\partial Z_{p}}{\partial Z_{b}}=\frac{\partial\left(Z_{b}+a_{13}\right)}{\partial Z_{b}}=1
\end{aligned}
$$

$\frac{\partial Z_{p}}{\partial \theta_{x}}=\frac{\partial\left(Z_{b}+a_{13}\right)}{\partial \theta_{x}}=\frac{\partial a_{11}}{\partial \theta_{x}}$

$$
\begin{aligned}
& =c \theta_{x} s \theta_{z} *\left(c \tau_{y} c \tau_{z} \rho c(\omega) s(\alpha)-c \tau_{y} s \tau_{z} \rho c(\omega) c(\alpha)+s \tau_{y} \rho s(\omega)+l x_{s}^{b}\right) \\
& +c \theta_{x} c \theta_{z}
\end{aligned}
$$$$
*\left(c \tau_{x} s \tau_{z} \rho c(\omega) s(\alpha)+s \tau_{x} s \tau_{y} c \tau_{z} \rho c(\omega) s(\alpha)+c \tau_{x} c \tau_{z} \rho c(\omega) c(\alpha)\right.
$$$$
\left.-s \tau_{x} s \tau_{y} s \tau_{z} \rho c(\omega) c(\alpha)+-s \tau_{x} c \tau_{y} \rho s(\omega)+l y_{s}^{b}\right)+s \theta_{x} s \theta_{y} c \theta_{z}
$$$$
*\left(c \tau_{y} c \tau_{z} \rho c(\omega) s(\alpha)-c \tau_{y} s \tau_{z} \rho c(\omega) c(\alpha)+s \tau_{y} \rho s(\omega)+l x_{s}^{b}\right)-s \theta s \theta_{y} s \theta_{z}
$$$$
*\left(c \tau_{x} s \tau_{z} \rho c(\omega) s(\alpha)+s \tau_{x} s \tau_{y} c \tau_{z} \rho c(\omega) s(\alpha)+c \tau_{x} c \tau_{z} \rho c(\omega) c(\alpha)\right.
$$$$
\left.-s \tau_{x} s \tau_{y} s \tau_{z} \rho c(\omega) c(\alpha)+-s \tau_{x} c \tau_{y} \rho s(\omega)+l y_{s}^{b}\right)-s \theta_{x} c \theta_{y} *\left(s \tau_{x} s \tau_{z} \rho c(\omega) s(\alpha)\right.
$$$$
-c \tau_{x} s \tau_{y} c \tau_{z} \rho c(\omega) s(\alpha)+s \tau_{x} c \tau_{z} \rho c(\omega) c(\alpha)+c \tau_{x} s \tau_{y} s \tau_{z} \rho c(\omega) c(\alpha)
$$$$
\left.+c \tau_{x} c \tau_{y} \rho s(\omega)+l z_{s}^{b}\right)
$$

$$
\begin{aligned}
\frac{\partial Z_{p}}{\partial \theta_{y}}=\frac{\partial\left(Z_{b}+\right.}{\partial \theta_{y}}=\frac{\partial a_{11}}{\partial \theta_{y}} & \\
& =-c \theta_{y} c \theta_{x} c \theta_{z} *\left(c \tau_{y} c \tau_{z} \rho c(\omega) s(\alpha)-c \tau_{y} s \tau_{z} \rho c(\omega) c(\alpha)+s \tau_{y} \rho s(\omega)+l x_{s}^{b}\right) \\
& +c \theta_{y} c \theta_{x} s \theta_{z} \\
& *\left(c \tau_{x} s \tau_{z} \rho c(\omega) s(\alpha)+s \tau_{x} s \tau_{y} c \tau_{z} \rho c(\omega) s(\alpha)+c \tau_{x} c \tau_{z} \rho c(\omega) c(\alpha)\right. \\
& \left.-s \tau_{x} s \tau_{y} s \tau_{z} \rho c(\omega) c(\alpha)+-s \tau_{x} c \tau_{y} \rho s(\omega)+l y_{s}^{b}\right)-s \theta_{y} c \theta_{x} *\left(s \tau_{x} s \tau_{z} \rho c(\omega) s(\alpha)\right. \\
& -c \tau_{x} s \tau_{y} c \tau_{z} \rho c(\omega) s(\alpha)+s \tau_{x} c \tau_{z} \rho c(\omega) c(\alpha)+c \tau_{x} s \tau_{y} s \tau_{z} \rho c(\omega) c(\alpha) \\
& \left.+c \tau_{x} c \tau_{y} \rho s(\omega)+l z_{s}^{b}\right)
\end{aligned}
$$




$$
\begin{aligned}
& \frac{\partial Z_{p}}{\partial \theta_{z}}=\frac{\partial\left(Z_{b}+a_{13}\right)}{\partial \theta_{z}}=\frac{\partial a_{11}}{\partial \theta_{z}} \\
& =c \theta_{z} s \theta_{x} *\left(c \tau_{y} c \tau_{z} \rho c(\omega) s(\alpha)-c \tau_{y} s \tau_{z} \rho c(\omega) c(\alpha)+s \tau_{y} \rho s(\omega)+l x_{s}^{b}\right) \\
& +s \theta_{z} c \theta_{x} s \theta_{y} *\left(c \tau_{y} c \tau_{z} \rho c(\omega) s(\alpha)-c \tau_{y} s \tau_{z} \rho c(\omega) c(\alpha)+s \tau_{y} \rho s(\omega)+l x_{s}^{b}\right) \\
& -s \theta_{z} s \theta_{x} \\
& *\left(c \tau_{x} s \tau_{z} \rho c(\omega) s(\alpha)+s \tau_{x} s \tau_{y} c \tau_{z} \rho c(\omega) s(\alpha)+c \tau_{x} c \tau_{z} \rho c(\omega) c(\alpha)\right. \\
& \left.-s \tau_{x} s \tau_{y} s \tau_{z} \rho c(\omega) c(\alpha)+-s \tau_{x} c \tau_{y} \rho s(\omega)+l y_{s}^{b}\right)+c \theta_{z} c \theta_{x} s \theta_{y} \\
& *\left(c \tau_{x} s \tau_{z} \rho c(\omega) s(\alpha)+s \tau_{x} s \tau_{y} c \tau_{z} \rho c(\omega) s(\alpha)+c \tau_{x} c \tau_{z} \rho c(\omega) c(\alpha)\right. \\
& \left.-s \tau_{x} s \tau_{y} s \tau_{z} \rho c(\omega) c(\alpha)+-s \tau_{x} c \tau_{y} \rho s(\omega)+l y_{s}^{b}\right) \\
& \frac{\partial Z_{p}}{\partial \rho}=\frac{\partial\left(Z_{b}+a_{13}\right)}{\partial \rho} \\
& =s \theta_{x} s \theta_{z} *\left(c \tau_{y} c \tau_{z} c(\omega) s(\alpha)-c \tau_{y} s \tau_{z} c(\omega) c(\alpha)+s \tau_{y} s(\omega)\right)-c \theta_{x} s \theta_{y} c \theta_{z} \\
& *\left(c \tau_{y} c \tau_{z} c(\omega) s(\alpha)-c \tau_{y} s \tau_{z} c(\omega) c(\alpha)+s \tau_{y} s(\omega)\right)+s \theta_{x} c \theta_{z} \\
& *\left(c \tau_{x} s \tau_{z} c(\omega) s(\alpha)+s \tau_{x} s \tau_{y} c \tau_{z} c(\omega) s(\alpha)+c \tau_{x} c \tau_{z} c(\omega) c(\alpha)\right. \\
& \left.-s \tau_{x} s \tau_{y} s \tau_{z} c(\omega) c(\alpha)+-s \tau_{x} c \tau_{y} s(\omega)\right)+c \theta_{x} s \theta_{y} s \theta_{z} \\
& *\left(c \tau_{x} s \tau_{z} c(\omega) s(\alpha)+s \tau_{x} s \tau_{y} c \tau_{z} c(\omega) s(\alpha)+c \tau_{x} c \tau_{z} c(\omega) c(\alpha)\right. \\
& \left.-s \tau_{x} s \tau_{y} s \tau_{z} c(\omega) c(\alpha)+-s \tau_{x} c \tau_{y} s(\omega)\right)+c \theta_{x} c \theta_{y} *\left(s \tau_{x} s \tau_{z} c(\omega) s(\alpha)\right. \\
& \left.-c \tau_{x} s \tau_{y} c \tau_{z} c(\omega) s(\alpha)+s \tau_{x} c \tau_{z} c(\omega) c(\alpha)+c \tau_{x} s \tau_{y} s \tau_{z} c(\omega) c(\alpha)+c \tau_{x} c \tau_{y} s(\omega)\right) \\
& \frac{\partial Z_{p}}{\partial \omega}=\frac{\partial\left(Z_{b}+a_{13}\right)}{\partial \omega} \\
& =s \theta_{x} s \theta_{z} *\left(-s \omega c \tau_{y} c \tau_{z} \rho c(\omega) s(\alpha)+s \omega c \tau_{y} s \tau_{z} \rho c(\omega) c(\alpha)+s \tau_{y} \rho c(\omega)\right) \\
& -c \theta_{x} s \theta_{y} c \theta_{z} *\left(-s \omega c \tau_{y} c \tau_{z} \rho c(\omega) s(\alpha)+s \omega c \tau_{y} s \tau_{z} \rho c(\omega) c(\alpha)+s \tau_{y} \rho c(\omega)\right) \\
& +s \theta_{x} c \theta_{z} \\
& *\left(-s \omega c \tau_{x} s \tau_{z} \rho c(\omega) s(\alpha)-s \omega s \tau_{x} s \tau_{y} c \tau_{z} \rho c(\omega) s(\alpha)-s \omega c \tau_{x} c \tau_{z} \rho c(\omega) c(\alpha)\right. \\
& \left.+s \omega s \tau_{x} s \tau_{y} s \tau_{z} \rho c(\omega) c(\alpha)-s \tau_{x} c \tau_{y} \rho c(\omega)\right)+c \theta_{x} s \theta_{y} s \theta_{z} \\
& *\left(-s \omega c \tau_{x} s \tau_{z} \rho c(\omega) s(\alpha)-s \omega s \tau_{x} s \tau_{y} c \tau_{z} \rho c(\omega) s(\alpha)-s \omega c \tau_{x} c \tau_{z} \rho c(\omega) c(\alpha)\right. \\
& \left.+s \omega s \tau_{x} s \tau_{y} s \tau_{z} \rho c(\omega) c(\alpha)-s \tau_{x} c \tau_{y} \rho c(\omega)\right)+c \theta_{x} c \theta_{y} *\left(-s \omega s \tau_{x} s \tau_{z} \rho c(\omega) s(\alpha)\right. \\
& +s \omega c \tau_{x} s \tau_{y} c \tau_{z} \rho c(\omega) s(\alpha)-s \omega s \tau_{x} c \tau_{z} \rho c(\omega) c(\alpha)-s \omega c \tau_{x} s \tau_{y} s \tau_{z} \rho c(\omega) c(\alpha) \\
& \left.+c \tau_{x} c \tau_{y} \rho c(\omega)\right)
\end{aligned}
$$




$$
\begin{aligned}
& \frac{\partial Z_{p}}{\partial \alpha}=\frac{\partial\left(Z_{b}+a_{13}\right)}{\partial \alpha} \\
& =s \theta_{x} s \theta_{z} *\left(c \tau_{y} c \tau_{z} \rho c(\omega) c(\alpha)+c \tau_{y} s \tau_{z} \rho c(\omega) s(\alpha)\right)-c \theta_{x} s \theta_{y} c \theta_{z} \\
& *\left(c \tau_{y} c \tau_{z} \rho c(\omega) c(\alpha)+c \tau_{y} s \tau_{z} \rho c(\omega) s(\alpha)\right)+s \theta_{x} c \theta_{z} \\
& *\left(c \tau_{x} s \tau_{z} \rho c(\omega) c(\alpha)+s \tau_{x} s \tau_{y} c \tau_{z} \rho c(\omega) c(\alpha)-c \tau_{x} c \tau_{z} \rho c(\omega) s(\alpha)\right. \\
& \left.+s \tau_{x} s \tau_{y} s \tau_{z} \rho c(\omega) s(\alpha)\right)+c \theta_{x} s \theta_{y} s \theta_{z} \\
& \text { * }\left(c \tau_{x} s \tau_{z} \rho c(\omega) c(\alpha)+s \tau_{x} s \tau_{y} c \tau_{z} \rho c(\omega) c(\alpha)-c \tau_{x} c \tau_{z} \rho c(\omega) s(\alpha)\right. \\
& \left.+s \tau_{x} s \tau_{y} s \tau_{z} \rho c(\omega) s(\alpha)\right)+c \theta_{x} c \theta_{y} *\left(s \tau_{x} s \tau_{z} \rho c(\omega) c(\alpha)-c \tau_{x} s \tau_{y} c \tau_{z} \rho c(\omega) c(\alpha)\right. \\
& \left.-s \tau_{x} c \tau_{z} \rho c(\omega) s(\alpha)-c \tau_{x} s \tau_{y} s \tau_{z} \rho c(\omega) s(\alpha)\right) \\
& \frac{\partial Z_{p}}{\partial \tau_{x}}=\frac{\partial\left(Z_{b}+a_{13}\right)}{\partial \tau_{x}} \\
& =s \theta_{x} c \theta_{z} \\
& *\left(-s \tau_{x} s \tau_{z} \rho c(\omega) s(\alpha)+c \tau_{x} s \tau_{y} c \tau_{z} \rho c(\omega) s(\alpha)-s \tau_{x} c \tau_{z} \rho c(\omega) c(\alpha)\right. \\
& \left.-c \tau_{x} s \tau_{y} s \tau_{z} \rho c(\omega) c(\alpha)+-c \tau_{x} c \tau_{y} \rho s(\omega)\right)+c \theta_{x} s \theta_{y} s \theta_{z} \\
& *\left(-s \tau_{x} s \tau_{z} \rho c(\omega) s(\alpha)+c \tau_{x} s \tau_{y} c \tau_{z} \rho c(\omega) s(\alpha)-s \tau_{x} c \tau_{z} \rho c(\omega) c(\alpha)\right. \\
& \left.-c \tau_{x} s \tau_{y} s \tau_{z} \rho c(\omega) c(\alpha)+-c \tau_{x} c \tau_{y} \rho s(\omega)\right)+c \theta_{x} c \theta_{y} *\left(c \tau_{x} s \tau_{z} \rho c(\omega) s(\alpha)\right. \\
& +s \tau_{x} s \tau_{y} c \tau_{z} \rho c(\omega) s(\alpha)+c \tau_{x} c \tau_{z} \rho c(\omega) c(\alpha)-s \tau_{x} s \tau_{y} s \tau_{z} \rho c(\omega) c(\alpha) \\
& \left.-s \tau_{x} c \tau_{y} \rho s(\omega)\right) \\
& \frac{\partial Z_{p}}{\partial \tau_{y}}=\frac{\partial\left(Z_{b}+a_{13}\right)}{\partial \tau_{y}} \\
& =s \theta_{x} s \theta_{z} *\left(-s \tau_{y} c \tau_{z} \rho c(\omega) s(\alpha)+s \tau_{y} s \tau_{z} \rho c(\omega) c(\alpha)+c \tau_{y} \rho s(\omega)\right)-c \theta_{x} s \theta_{y} c \theta_{z} \\
& *\left(-c \tau_{y} c \tau_{z} \rho c(\omega) s(\alpha)+c \tau_{y} s \tau_{z} \rho c(\omega) c(\alpha)-s \tau_{y} \rho s(\omega)\right)+s \theta_{x} c \theta_{z} \\
& *\left(c \tau_{y} s \tau_{x} c \tau_{z} \rho c(\omega) s(\alpha)-c \tau_{y} s \tau_{x} s \tau_{z} \rho c(\omega) c(\alpha)+s \tau_{y} s \tau_{x} \rho s(\omega)\right)+c \theta_{x} s \theta_{y} s \theta_{z} \\
& *\left(c \tau_{y} s \tau_{x} c \tau_{z} \rho c(\omega) s(\alpha)-c \tau_{y} s \tau_{x} s \tau_{z} \rho c(\omega) c(\alpha)+s \tau_{y} s \tau_{x} \rho s(\omega)\right)+c \theta_{x} c \theta_{y} \\
& *\left(-c \tau_{y} c \tau_{x} c \tau_{z} \rho c(\omega) s(\alpha)+c \tau_{y} c \tau_{x} s \tau_{z} \rho c(\omega) c(\alpha)-s \tau_{y} c \tau_{x} \rho s(\omega)\right)
\end{aligned}
$$




$$
\begin{aligned}
& \frac{\partial Z_{p}}{\partial \tau_{z}}=\frac{\partial\left(Z_{b}+\right.}{\partial \tau_{z}}\left.a_{13}\right) \\
&=s \theta_{x} s \theta_{z} *\left(-s \tau_{z} c \tau_{y} \rho c(\omega) s(\alpha)-c \tau_{y} c \tau_{z} \rho c(\omega) c(\alpha)\right)-c \theta_{x} s \theta_{y} c \theta_{z} \\
& *\left(-s \tau_{z} c \tau_{y} \rho c(\omega) s(\alpha)-c \tau_{z} c \tau_{y} \rho c(\omega) c(\alpha)\right)+s \theta_{x} c \theta_{z} \\
& *\left(c \tau_{z} c \tau_{x} \rho c(\omega) s(\alpha)-s \tau_{z} s \tau_{x} s \tau_{y} \rho c(\omega) s(\alpha)-s \tau_{z} c \tau_{x} \rho c(\omega) c(\alpha)\right. \\
&\left.-c \tau_{z} s \tau_{x} s \tau_{y} \rho c(\omega) c(\alpha)\right)+c \theta_{x} s \theta_{y} s \theta_{z} \\
& *\left(c \tau_{z} c \tau_{x} \rho c(\omega) s(\alpha)-s \tau_{z} s \tau_{x} s \tau_{y} \rho c(\omega) s(\alpha)-s \tau_{z} c \tau_{x} \rho c(\omega) c(\alpha)\right. \\
&\left.+c \tau_{z} s \tau_{x} s \tau_{y} \rho c(\omega) c(\alpha)\right)+c \theta_{x} c \theta_{y} *\left(c \tau_{z} s \tau_{x} \rho c(\omega) s(\alpha)+s \tau_{z} c \tau_{x} s \tau_{y} \rho c(\omega) s(\alpha)\right. \\
&\left.-s \tau_{z} s \tau_{x} \rho c(\omega) c(\alpha)+c \tau_{z} c \tau_{x} s \tau_{y} \rho c(\omega) c(\alpha)\right) \\
& \\
& \frac{\partial Z_{p}}{\partial l x_{s}^{b}}=\frac{\partial\left(Z_{b}+a_{13}\right)}{\partial l x_{s}^{b}}=\frac{\partial a_{13}}{\partial l x_{s}^{b}}=s \theta_{x} s \theta_{z}-c \theta_{x} s \theta_{y} c \theta_{z} \\
& \frac{\partial Z_{p}}{\partial l y_{s}^{b}}=\frac{\partial\left(Z_{b}+a_{13}\right)}{\partial l y_{s}^{b}}=\frac{\partial a_{13}}{\partial l y_{s}^{b}}=s \theta_{x} c \theta_{z}+c \theta_{x} s \theta_{y} s \theta_{z} \\
& \frac{\partial Z_{p}}{\partial l z_{s}^{b}}=\frac{\partial\left(Z_{b}+a_{13}\right)}{\partial l z_{s}^{b}}=\frac{\partial a_{13}}{\partial l z_{s}^{b}}=c \theta_{x} c \theta_{y}
\end{aligned}
$$


Thus, the Jacobian matrix elements evaluated at the initial values yields:

For $X_{p}$ :

$$
\begin{aligned}
& \left.\frac{\partial X_{p}}{\partial X_{b}}\right|_{X_{b_{0}}}=1 \\
& \left.\frac{\partial X_{p}}{\partial Y_{b}}\right|_{Y_{b_{0}}}=0 \\
& \left.\frac{\partial X_{p}}{\partial Z_{b}}\right|_{Z_{b_{0}}}=0 \\
& \left.\frac{\partial X_{p}}{\partial \theta_{x}}\right|_{\theta_{x_{0}}}=0 \\
& \left.\frac{\partial X_{p}}{\partial \theta_{y}}\right|_{\theta_{y_{0}}}=0 \\
& \left.\frac{\partial X_{p}}{\partial \theta_{z}}\right|_{\theta_{z_{0}}}=-100 \\
& \left.\frac{\partial X_{p}}{\partial \rho}\right|_{\rho_{0}}=0 \\
& \left.\frac{\partial X_{p}}{\partial \omega}\right|_{\omega_{0}}=0 \\
& \left.\frac{\partial X_{p}}{\partial \alpha}\right|_{\alpha_{0}}=100 \\
& \left.\frac{\partial X_{p}}{\partial \tau_{x}}\right|_{\tau_{x_{0}}}=0 \\
& \left.\frac{\partial X_{p}}{\partial \tau_{y}}\right|_{\tau_{y_{0}}}=0 \\
& \left.\frac{\partial X_{p}}{\partial \tau_{z}}\right|_{\tau_{z 0}}=-100 \\
& \left.\frac{\partial X_{p}}{\partial l x_{s}^{b}}\right|_{l x_{s_{0}}^{b}}=1 \\
& \left.\frac{\partial X_{p}}{\partial l y_{s}^{b}}\right|_{l y_{s_{0}}^{b}}=0
\end{aligned}
$$




$$
\left.\frac{\partial X_{p}}{\partial l z_{s}^{b}}\right|_{l z_{s_{0}}^{b}}=0
$$

For $Y_{p}$ :

$$
\begin{aligned}
& \left.\frac{\partial Y_{p}}{\partial X_{b}}\right|_{X_{b_{0}}}=0 \\
& \left.\frac{\partial Y_{p}}{\partial Y_{b}}\right|_{Y_{b_{0}}}=1 \\
& \left.\frac{\partial Y_{p}}{\partial Z_{b}}\right|_{Z_{b_{0}}}=0 \\
& \left.\frac{\partial Y_{p}}{\partial \theta_{x}}\right|_{\theta_{x_{0}}}=0 \\
& \left.\frac{\partial Y_{p}}{\partial \theta_{y}}\right|_{\theta_{y_{0}}}=0 \\
& \left.\frac{\partial Y_{p}}{\partial \theta_{z}}\right|_{\theta_{z 0}}=0 \\
& \left.\frac{\partial Y_{p}}{\partial \rho}\right|_{\rho_{0}}=1 \\
& \left.\frac{\partial Y_{p}}{\partial \omega}\right|_{\omega_{0}}=0 \\
& \left.\frac{\partial Y_{p}}{\partial \alpha}\right|_{\alpha_{0}}=0 \\
& \left.\frac{\partial Y_{p}}{\partial \tau_{x}}\right|_{\tau_{x_{0}}}=0 \\
& \left.\frac{\partial Y_{p}}{\partial \tau_{y}}\right|_{\tau_{y_{0}}}=0 \\
& \left.\frac{\partial Y_{p}}{\partial \tau_{z}}\right|_{\tau_{z 0}}=-100 \\
& \left.\frac{\partial Y_{p}}{\partial l x_{s}^{b}}\right|_{l x_{s_{0}}^{b}}=0 \\
& \left.\frac{\partial Y_{p}}{\partial l y_{s}^{b}}\right|_{l y_{s_{0}}^{b}}=1 \\
& \left.\frac{\partial Y_{p}}{\partial l z_{s}^{b}}\right|_{l l_{s_{0}}^{b}}=0
\end{aligned}
$$


For $Z_{p}$ :

$$
\begin{aligned}
& \left.\frac{\partial Z_{p}}{\partial X_{b}}\right|_{X_{b_{0}}}=0 \\
& \left.\frac{\partial Z_{p}}{\partial Y_{b}}\right|_{Y_{b_{0}}}=0 \\
& \left.\frac{\partial Z_{p}}{\partial Z_{b}}\right|_{Z_{b_{0}}}=1 \\
& \left.\frac{\partial Z_{p}}{\partial \theta_{x}}\right|_{\theta_{x_{0}}}=100 \\
& \left.\frac{\partial Z_{p}}{\partial \theta_{y}}\right|_{\theta_{y_{0}}}=0 \\
& \left.\frac{\partial Z_{p}}{\partial \theta_{z}}\right|_{\theta_{z 0}}=0 \\
& \left.\frac{\partial Z_{p}}{\partial \rho}\right|_{\rho_{0}}=0 \\
& \left.\frac{\partial Z_{p}}{\partial \omega}\right|_{\omega_{0}}=100 \\
& \left.\frac{\partial Z_{p}}{\partial \alpha}\right|_{\alpha_{0}}=0 \\
& \left.\frac{\partial Z_{p}}{\partial \tau_{x}}\right|_{\tau_{x_{0}}}=0 \\
& \left.\frac{\partial Z_{p}}{\partial \tau_{y}}\right|_{\tau_{y_{0}}}=0 \\
& \left.\frac{\partial Z_{p}}{\partial \tau_{z}}\right|_{\tau_{z 0}}=-100 \\
& \left.\frac{\partial Z_{p}}{\partial l x_{s}^{b}}\right|_{l x_{s_{0}}^{b}}=0 \\
& \left.\frac{\partial Z_{p}}{\partial l y_{s}^{b}}\right|_{l y_{s_{0}}^{b}}=0 \\
& \left.\frac{\partial Z_{p}}{\partial l z_{s}^{b}}\right|_{l z_{s_{0}}^{b}}=1
\end{aligned}
$$




\section{References}

[1] Shan, J., \& Toth, C. K. (2018). Topographic laser ranging and scanning: principles and processing. CRC press.

[2] Lin, Y. C., Cheng, Y. T., Lin, Y. J., Flatt, J. E., Habib, A., \& Bullock, D. (2019). Evaluating the Accuracy of Mobile LiDAR for Mapping Airfield Infrastructure. Transportation Research Record, 2673(4), 117-124.

[3] Wehr, A., \& Lohr, U. (1999). Airborne laser scanning - an introduction and overview. ISPRS Journal of photogrammetry and remote sensing, 54(2-3), 68-82.

[4] Yan, W. Y., \& Shaker, A. (2018). Airborne LiDAR intensity banding: Cause and solution. ISPRS journal of photogrammetry and remote sensing, 142, 301-310.

[5] Velodyne Lidar. Velodyne Lidar sensors .Retrieved from https://velodynelidar.com/?gclid=EAlalQobChMImrfuraKv5gIVDvDACh0dMgWKEAAYASAAEgL75PD Bw $\underline{\mathrm{E}}$

[6] Quanergy. Quanergy LiDAR sensors. Retrieved from quanragy https://quanergy.com/

[7] Poulton, C. V., Yaacobi, A., Cole, D. B., Byrd, M. J., Raval, M., Vermeulen, D., \& Watts, M. R. (2017). Coherent solid-state LIDAR with silicon photonic optical phased arrays. Optics letters, 42(20), 4091-4094.

[8] Leddartech correspondence, Technical support, Phone call (October $4^{\text {th }}, 2018$ )

[9] Quanargy correspondence, Chai Khoche, Phone call (July $\left.8^{\text {th }}, 2019\right)$

[10] Puente, I., González-Jorge, H., Martínez-Sánchez, J., \& Arias, P. (2013). Review of mobile mapping and surveying technologies. Measurement, 46(7), 2127-2145.

[11] Che, E., Jung, J., \& Olsen, M. J. (2019). Object recognition, segmentation, and classification of mobile laser scanning point clouds: A state of the art review. Sensors, 19(4), 810.

[12] Julge, K., Ellmann, A., Vajakas, T., \& Kolka, R. (2016). Initial tests and accuracy assessment of a compact mobile laser scanning system. International Archives of the Photogrammetry, Remote Sensing \& Spatial Information Sciences, 41.

[13] Sairam, N., Nagarajan, S., \& Ornitz, S. (2016). Development of mobile mapping system for $3 \mathrm{~d}$ road asset inventory. Sensors, 16(3), 367.

[14] Julge, K., Vajakas, T., \& Ellmann, A. (2017). Performance analysis of a compact and lowcost mapping-grade mobile laser scanning system. Journal of Applied Remote Sensing, 11(4), 044003.

[15] Shamseldin, T. (2018). System Architecture, Calibration, and Control for LiDAR Systems Onboard Unmanned Vehicles (Doctoral dissertation, Purdue University). 
[16] Rehak, M. (2017). Integrated sensor orientation on micro aerial vehicles (No. THESIS). EPFL.

[17] Chapman, A. (2016). Types of drones: Multi-rotor vs fixed-wing vs single rotor vs hybrid VTOL. Australian UAV.

[18] Li, J., Yang, B., Cong, Y., Li, S., \& Yue, Y. (2019). Integration of a Low-Cost Multisensory Uav System for Forest Application. International Archives of the Photogrammetry, Remote Sensing and Spatial Information Sciences, 42(2/W13).

[19] Li, J., Yang, B., Cong, Y., Cao, L., Fu, X., \& Dong, Z. (2019). 3D Forest Mapping Using A Low-Cost UAV Laser Scanning System: Investigation and Comparison. Remote Sensing, 11(6), 717.

[20] Christiansen, M., Laursen, M., Jørgensen, R., Skovsen, S., \& Gislum, R. (2017). Designing and testing a UAV mapping system for agricultural field surveying. Sensors, 17(12), 2703.

[21] Teng, G. E., Zhou, M., Li, C. R., Wu, H. H., Li, W., Meng, F. R., ... \& Ma, L. (2017). MiniUAV LiDAR for power line inspection. Int. Arch. Photogramm. Remote Sens. Spat. Inf. Sci, 297300.

[22] Phoenixlidar. Phoenixlidar LiDAR mapping systems. Retrieved from https://www.phoenixlidar.com/

[23] Routescene. Routescene LiDAR mapping systems. Retrieved from https://www.routescene.com/

[24] Yellowscan. Yellowscan LiDAR mapping systems. Retrieved from https://www.yellowscan$\underline{\text { lidar.com/ }}$

[25] Riegel. Riegl Vux-240 LiDAR scanner. Retrieved from http://www.riegl.com/products/unmanned-scanning/riegl-vux-240

[26] Disney, M., Burt, A., Calders, K., Schaaf, C., \& Stovall, A. (2019). Innovations in ground and airborne technologies as reference and for training and validation: terrestrial laser scanning (TLS). Surveys in Geophysics, 40(4), 937-958.

[27] Trimble. Trimble-sx10. Retrieved from https://geospatial.trimble.com/products-andsolutions/trimble-sx 10

[28] Faro. Faro Focus LiDAR scanner. Retrieved from https://www.faro.com/products/construction-bim/faro-focus/

[29] Teledyneoptech. Polaris Laser Scanner. Retrieved from http://www.teledyneoptech.com/en/products/static-3d-survey/polaris/

[30] Leica geosystems. BLK360 LiDAR scanner. Retrieved from https://shop.leicageosystems.com/ 
[31] Cahalane, C., Lewis, P., McElhinney, C., \& McCarthy, T. (2015). Optimising mobile mapping system laser scanner orientation. ISPRS International Journal of Geo-Information, 4(1), 302-319.

[32] Rieger, P., Studnicka, N., Pfennigbauer, M., \& Ullrich, A. (2010). Advances in mobile laser scanning data acquisition. FIG Congresss.

[33] Cahalane, C., Lewis, P., McElhinney, C. P., McNerney, E., \& McCarthy, T. (2016). Improving mms performance during infrastructure surveys through geometry aided design. Infrastructures, 1(1), 5.

[34] Velas, M., Spanel, M., \& Herout, A. (2016, May). Collar line segments for fast odometry estimation from velodyne point clouds. In 2016 IEEE International Conference on Robotics and Automation (ICRA) (pp. 4486-4495). IEEE.

[35] Smith, M. W., Carrivick, J. L., \& Quincey, D. J. (2016). Structure from motion photogrammetry in physical geography. Progress in Physical Geography, 40(2), 247-275.

[36] Longuet-Higgins, H. C. (1981). A computer algorithm for reconstructing a scene from two projections. Nature, 293(5828), 133.

[37] Granshaw, S. I., \& Fraser, C. S. (2015). Computer vision and photogrammetry: Interaction or introspection?. The Photogrammetric Record, 30(149), 3-7.

[38] Hartley, R. I. (1997). In defense of the eight-point algorithm. IEEE Transactions on pattern analysis and machine intelligence, 19(6), 580-593.

[39] Westoby, M. J., Brasington, J., Glasser, N. F., Hambrey, M. J., \& Reynolds, J. M. (2012). 'Structure-from-Motion'photogrammetry: A low-cost, effective tool for geoscience applications. Geomorphology, 179, 300-314.

[40] Lowe, D. G. (2004). Distinctive image features from scale-invariant keypoints. International journal of computer vision, 60(2), 91-110.

[41] Bay, H., Ess, A., Tuytelaars, T., \& Van Gool, L. (2008). Speeded-up robust features (SURF). Computer vision and image understanding, 110(3), 346-359.

[42] Calonder, M., Lepetit, V., Strecha, C., \& Fua, P. (2010, September). Brief: Binary robust independent elementary features. In European conference on computer vision (pp. 778-792). Springer, Berlin, Heidelberg.

[43] Rublee, E., Rabaud, V., Konolige, K., \& Bradski, G. R. (2011, November). ORB: An efficient alternative to SIFT or SURF. In ICCV (Vol. 11, No. 1, p. 2).

[44] Furukawa, Y., \& Ponce, J. (2009). Accurate, dense, and robust multiview stereopsis. IEEE transactions on pattern analysis and machine intelligence, 32(8), 1362-1376.

[45] Snavely, N., Seitz, S. M., \& Szeliski, R. (2008). Modeling the world from internet photo collections. International journal of computer vision, 80(2), 189-210. 
[47] Eltner, A., Kaiser, A., Castillo, C., Rock, G., Neugirg, F., \& Abellán, A. (2016). Image-based surface reconstruction in geomorphometry-merits, limits and developments. Earth Surface Dynamics, 4(2), 359-389.

[48] Remondino, F., Spera, M. G., Nocerino, E., Menna, F., \& Nex, F. (2014). State of the art in high density image matching. The photogrammetric record, 29(146), 144-166.

[49] Sona, G., Pinto, L., Pagliari, D., Passoni, D., \& Gini, R. (2014). Experimental analysis of different software packages for orientation and digital surface modelling from UAV images. Earth Science Informatics, 7(2), 97-107.

[50] Fonstad, M. A., Dietrich, J. T., Courville, B. C., Jensen, J. L., \& Carbonneau, P. E. (2013). Topographic structure from motion: a new development in photogrammetric measurement. Earth Surface Processes and Landforms, 38(4), 421-430.

[51] Salach, A., Bakuła, K., Pilarska, M., Ostrowski, W., Górski, K., \& Kurczyński, Z. (2018). Accuracy assessment of point clouds from LidaR and dense image matching acquired using the UAV platform for DTM creation. ISPRS International Journal of Geo-Information, 7(9), 342.

[52] Harwin, S., \& Lucieer, A. (2012). Assessing the accuracy of georeferenced point clouds produced via multi-view stereopsis from unmanned aerial vehicle (UAV) imagery. Remote Sensing, 4(6), 1573-1599.

[53] Zieher, T., Toschi, I., Remondino, F., Rutzinger, M., Kofler, C., Mejia-Aguillar, A., \& Schlögel, R. (2018). Sensor-and scene-guided integration of TLS and photogrammetric point clouds for landslide monitoring. ISPRS.

[54] Gneeniss, A. S., Mills, J. P., \& Miller, P. E. (2015). In-flight photogrammetric camera calibration and validation via complementary lidar. ISPRS journal of photogrammetry and remote sensing, 100, 3-13.

[55] Lin, C. C., Tai, Y. C., Lee, J. J., \& Chen, Y. S. (2017). A novel point cloud registration using 2D image features. EURASIP Journal on Advances in Signal Processing, 2017(1), 5.

[56] Persad, R. A., \& Armenakis, C. (2016). Co-registration of DSMs generated by UAV and terrestrial laser scanning systems. The International Archives of Photogrammetry, Remote Sensing and Spatial Information Sciences, 41, 985.

[57] Rizeei, H. M., \& Pradhan, B. (2019). Urban Mapping Accuracy Enhancement in High-Rise Built-Up Areas Deployed by 3D-Orthorectification Correction from WorldView-3 and LiDAR Imageries. Remote Sensing, 11(6), 692.

[58] Yao, L., Wu, H., Li, Y., Meng, B., Qian, J., Liu, C., \& Fan, H. (2017). Registration of vehicleborne point clouds and panoramic images based on sensor constellations. Sensors, 17(4), 837.

[59] Javanmardi, M., Javanmardi, E., Gu, Y., \& Kamijo, S. (2017). Towards high-definition 3D urban mapping: Road feature-based registration of mobile mapping systems and aerial imagery. Remote Sensing, 9(10), 975. 
[60] Zhang, S., Tao, P., Wang, L., Hou, Y., \& Hu, Z. (2019). Improving Details of Building Façades in Open LiDAR Data Using Ground Images. Remote Sensing, 11(4), 420.

[61] Berrio, J. S., Ward, J., Worrall, S., Zhou, W., \& Nebot, E. (2017). Fusing Lidar and Semantic Image Information in Octree Maps. In ACRA Australasian Conference on Robotics and Automation 2017.

[62] Habib, A., Ghanma, M., Morgan, M., \& Al-Ruzouq, R. (2005). Photogrammetric and LiDAR data registration using linear features. Photogrammetric Engineering \& Remote Sensing, 71(6), 699-707.

[63] Toschi, I., Remondino, F., Rothe, R., \& Klimek, K. (2018). COMBINING AIRBORNE OBLIQUE CAMERA AND LIDAR SENSORS: INVESTIGATION AND NEW PERSPECTIVES. International Archives of the Photogrammetry, Remote Sensing \& Spatial Information Sciences.

[64] Eltner, A., Kaiser, A., Castillo, C., Rock, G., Neugirg, F., \& Abellán, A. (2016). Image-based surface reconstruction in geomorphometry-merits, limits and developments. Earth Surface Dynamics, 4(2), 359-389.

[65] Shaad, K., Ninsalam, Y., Padawangi, R., \& Burlando, P. (2016). Towards high resolution and cost-effective terrain mapping for urban hydrodynamic modelling in densely settled rivercorridors. Sustainable Cities and Society, 20, 168-179.

[66] Pineux, N., Lisein, J., Swerts, G., Bielders, C. L., Lejeune, P., Colinet, G., \& Degré, A. (2017). Can DEM time series produced by UAV be used to quantify diffuse erosion in an agricultural watershed?. Geomorphology, 280, 122-136.

[67] Chen, Z., Gao, B., \& Devereux, B. (2017). State-of-the-art: DTM generation using airborne LIDAR data. Sensors, 17(1), 150.

[68] Mancini, F., Dubbini, M., Gattelli, M., Stecchi, F., Fabbri, S., \& Gabbianelli, G. (2013). Using unmanned aerial vehicles (UAV) for high-resolution reconstruction of topography: The structure from motion approach on coastal environments. Remote Sensing, 5(12), 6880-6898.

[69] Xu, Z., Wu, L., Gerke, M., Wang, R., \& Yang, H. (2016). Skeletal camera network embedded structure-from-motion for 3D scene reconstruction from UAV images. ISPRS journal of photogrammetry and remote sensing, 121, 113-127.

[70] Su, T. C., \& Chou, H. T. (2015). Application of multispectral sensors carried on unmanned aerial vehicle (UAV) to trophic state mapping of small reservoirs: a case study of Tain-Pu reservoir in Kinmen, Taiwan. Remote Sensing, 7(8), 10078-10097.

[71] Webster, C., Bühler, Y., Schirmer, M., Stoffel, A., Giulia, M., \& Jonas, T. (2017, December). Mapping snow depth distribution in forested terrain using unmanned aerial vehicles and structurefrom-motion. In AGU Fall Meeting Abstracts.

[72] López, J. B., Jiménez, G. A., Romero, M. S., García, E. A., Martín, S. F., Medina, A. L., \& Guerrero, J. E. (2016). 3D modelling in archaeology: The application of Structure from Motion 
methods to the study of the megalithic necropolis of Panoria (Granada, Spain). Journal of archaeological science: reports, 10, 495-506.

[73] Shean, D. E., Alexandrov, O., Moratto, Z. M., Smith, B. E., Joughin, I. R., Porter, C., \& Morin, P. (2016). An automated, open-source pipeline for mass production of digital elevation models (DEMs) from very-high-resolution commercial stereo satellite imagery. ISPRS Journal of Photogrammetry and Remote Sensing, 116, 101-117.

[74] Di Rita, M., Nascetti, A., \& Crespi, M. (2017). Open source tool for DSMs generation from high resolution optical satellite imagery: development and testing of an OSSIM plugin. International journal of remote sensing, 38(7), 1788-1808.

[75] Casella, E., Collin, A., Harris, D., Ferse, S., Bejarano, S., Parravicini, V., ... \& Rovere, A. (2017). Mapping coral reefs using consumer-grade drones and structure from motion photogrammetry techniques. Coral Reefs, 36(1), 269-275.

[76] Rizki, P. N. M., Eum, J., Lee, H., \& Oh, S. (2017). Spark-based in-memory DEM creation from 3D LiDAR point clouds. Remote sensing letters, 8(4), 360-369.

[77] Gillin, C. P., Bailey, S. W., McGuire, K. J., \& Prisley, S. P. (2015). Evaluation of LiDARderived DEMs through terrain analysis and field comparison. Photogrammetric Engineering \& Remote Sensing, 81(5), 387-396.

[78] Su, T. C., \& Chou, H. T. (2015). Application of multispectral sensors carried on unmanned aerial vehicle (UAV) to trophic state mapping of small reservoirs: a case study of Tain-Pu reservoir in Kinmen, Taiwan. Remote Sensing, 7(8), 10078-10097.

[79] Guo, Q., Li, W., Yu, H., \& Alvarez, O. (2010). Effects of topographic variability and lidar sampling density on several DEM interpolation methods. Photogrammetric Engineering \& Remote Sensing, 76(6), 701-712.

[80] Mousa, Y. A., Helmholz, P., Belton, D., \& Bulatov, D. (2019). Building detection and regularisation using DSM and imagery information. The Photogrammetric Record, 34(165), 85107.

[81] Koutsoudis, A., Vidmar, B., Ioannakis, G., Arnaoutoglou, F., Pavlidis, G., \& Chamzas, C. (2014). Multi-image 3D reconstruction data evaluation. Journal of Cultural Heritage, 15(1), 7379.

[82] Harwin, S., \& Lucieer, A. (2012). Assessing the accuracy of georeferenced point clouds produced via multi-view stereopsis from unmanned aerial vehicle (UAV) imagery. Remote Sensing, 4(6), 1573-1599.

[83] Leitão, J. P., Moy de Vitry, M., Scheidegger, A., \& Rieckermann, J. (2016). Assessing the quality of digital elevation models obtained from mini unmanned aerial vehicles for overland flow modelling in urban areas. Hydrology and Earth System Sciences, 20(4), 1637-1653. 
[84] Ouédraogo, M. M., Degré, A., Debouche, C., \& Lisein, J. (2014). The evaluation of unmanned aerial system-based photogrammetry and terrestrial laser scanning to generate DEMs of agricultural watersheds. Geomorphology, 214, 339-355.

[85] Zarco-Tejada, P. J., Diaz-Varela, R., Angileri, V., \& Loudjani, P. (2014). Tree height quantification using very high resolution imagery acquired from an unmanned aerial vehicle (UAV) and automatic 3D photo-reconstruction methods. European journal of agronomy, 55, 8999.

[86] De Reu, J., Trachet, J., Laloo, P., \& De Clercq, W. (2016). From low cost UAV survey to high resolution topographic data: Developing our understanding of a medieval outport of Bruges. Archaeological Prospection, 23(4), 335-346.

[87] Ajayi, O. G., Salubi, A. A., Angbas, A. F., \& Odigure, M. G. (2017). Generation of accurate digital elevation models from UAV acquired low percentage overlapping images. International journal of remote sensing, 38(8-10), 3113-3134.

[88] James, M. R., Robson, S., d'Oleire-Oltmanns, S., \& Niethammer, U. (2017). Optimising UAV topographic surveys processed with structure-from-motion: Ground control quality, quantity and bundle adjustment. Geomorphology, 280, 51-66.

[89] Deppe, O., Dorner, G., König, S., Martin, T., Voigt, S., \& Zimmermann, S. (2017). MEMS and FOG technologies for tactical and navigation grade inertial sensors - recent improvements and comparison. Sensors, 17(3), 567.

[90] Zhang, W., Qi, J., Wan, P., Wang, H., Xie, D., Wang, X., \& Yan, G. (2016). An easy-to-use airborne LiDAR data filtering method based on cloth simulation. Remote Sensing, 8(6), 501.

[91] Deppe, O., Dorner, G., König, S., Martin, T., Voigt, S., \& Zimmermann, S. (2017). MEMS and FOG technologies for tactical and navigation grade inertial sensors - recent improvements and comparison. Sensors, 17(3), 567.

[92] Guinamard, A., Ellipse, A. H. R. S., \& Performance, I. H. (2014). Miniature Inertial Sensors User Manual. SBG Systems, Rueil-Malmaison, France.

[93] Hexagon Positioning Intelligence. Novatel PwrPak7D-E1. Retrieved from https:/www.novatel.com/products/span-gnss-inertial-systems/span-combinedsystems/pwrpak7d-e1/

[94] Applanix. Applanix-15. Retrieved from https://www.applanix.com/products/dg-uavs.htm

[95] Antlgravity batteries,. XP-19 Retrieved from https://antigravitybatteries.com/products/microstarts/xp-10/

[96] Tallentcell, Tallentcell Retrieved from https://talentcell.com/products/12v-battery/

[97] May' ${ }^{1}$, N. C., \& Toth, C. K. (2007). Point positioning accuracy of airborne LiDAR systems: A rigorous analysis. International Archives of Photogrammetry, Remote Sensing and Spatial Information Sciences, Munich, Germany, 19-21. 
[98] Glennie, C. (2007). Rigorous 3D error analysis of kinematic scanning LIDAR systems. Journal of Applied Geodesy jag, 1(3), 147-157.

[99] Glennie, C., Kusevic, K., \& Mrstik, P. (2006, November). Performance analysis of a kinematic terrestrial LIDAR scanning system. In Proceedings of MAPPS/ASPRS Fall Conference, San Antonio, TX.

[100] Leslar, M., Hu, B., \& Wang, J. G. (2014). Error analysis of a mobile terrestrial LiDAR system. Geomatica, 68(3), 183-194.

[101] Pomerleau, F., Colas, F., \& Siegwart, R. (2015). A review of point cloud registration algorithms for mobile robotics. Foundations and Trends ${ }^{\circledR}$ in Robotics, 4(1), 1-104. 